\title{
A checklist for identifying Meliolales species
}

\section{Zeng $X Y^{1,2,3}$, Zhao $J^{1}$, Hongsanan $S^{2}$, Chomnunti $P^{2,3}$, Boonmee $S^{2}$, and Wen $\mathrm{TC}^{1^{*}}$}

\author{
${ }^{1}$ The Engineering Research Center of Southwest Bio-Pharmaceutical Resources, Ministry of Education, Guizhou \\ University, Guiyang 550025, China \\ ${ }^{2}$ Center of Excellence in Fungal Research, Mae Fah Luang University, Chiang Rai 57100, Thailand \\ ${ }^{3}$ School of Science, Mae Fah Luang University, Chiang Rai 57100, Thailand
}

Zeng XY, Zhao JJ, Hongsanan S, Chomnunti P, Boonmee S, Wen TC 2017 - A checklist for identifying Meliolales species. Mycosphere 8(1), 218-359, Doi 10.5943/mycosphere/8/1/16

\begin{abstract}
Meliolales is the largest order of epifoliar fungi, characterized by branched, dark brown, superficial mycelium with two-celled hyphopodia; superficial, globose to subglobose, dark brown perithecia, and septate, dark brown ascospores. The assumption of host-specificity means this group a highly diverse and it is imperative to identify the host before attempting to identify a fungal collection. This paper has compiled information from fungal and plant databases, including all fungal species of Meliolales and their host information from protologues. Current names of plants and corresponding fungi with integration of their synonyms are made into an alphabetical checklist, and references are provided. Exclusions and spelling conflicts of fungal species are also listed. Statistics show that the order comprises 2403 species (including 106 uncertain species), infecting among 194 host families, with an additional 20 excluded records. This checklist will be useful for the future identifications and classifications of Meliolales.
\end{abstract}

Key words - Armatellaceae - host range - identification - Meliolaceae - parasite - synonym

\section{Introduction}

Meliolales comprises nine genera distributed in the families Armatellaceae and Meliolaceae (Hongsanan et al. 2015). Armatellaceae comprises a monotypic genus Armatella, which is characterized by superficial, dark brown hyphae with hyphopodia, lacking phialides, superficial perithecia form on black colonies, 4-8-spored asci, and 1-septate, hyaline to brown ascospores (Hosagoudar 2003, Hongsanan et al. 2015). Meliolaceae was established to incorporate the epiphytic fungal genera Amazonia, Appendiculella, Asteridiella, Cryptomeliola, Endomeliola, Irenopsis, Meliola (generic type) and Setameliola (Martin 1941, Hongsanan et al. 2015, Reynolds 2010). 
Members of the family are characterized by branched, dark brown, superficial mycelium with phialides and two-celled hyphopodia; superficial, globose to subglobose, dark brown perithecial, 24-spored, evanescent asci, and 3-4-septate, dark brown, cylindrical ascospores (Fig. 1).

Meliolales is a biotrophic fungal group that occurs on leaves, petioles, twigs and rarely on fruits (Hansford 1961, Nayar et al. 1998, Saenz \& Taylor 1999, Old et al. 2003,). Species are more commonly distributed during the cool season in scrubs or open park-lands in tropical and subtropical regions that are subject to long annual dry periods (Hansford 1961, Goos \& Andersons 1974, Hongsanan et al. 2015, Rodríguez Justavino et al. 2015). On the other hand, they are absent in arid regions, which indicates a minimum water-requirement or humidity level is needed for their development (Hansford 1961).

Species in this order produce hyphopodia that penetrate the leaf surface to gain nutrients from host plants and results in a reduction of chlorophyll, starch, sugar, protein and aminoacid, however without causing pathogenic damage (Hosagoudar et al. 1997b, Old et al. 2003, Rodríguez Justavino $\&$ Piepenbring 2007). They could reduce photosynthesis by covering the host surface, and increase the temperature and respiration in those areas (Hosagoudar et al. 1997b, Hosagoudar \& Raju 2013, Hongsanan et al. 2015), but generally plants can photo-compensate for the light lost to epiphyll cover by increasing concentrations of chlorophyll (Anthony et al. 2002, Gilbert et al. 2007). Speicies of Meliolales can cause significant effects on crops, such as reducing yield and quality of fruits of Citrus (e.g. Meliola butleri, M. camelliae and M. citricola), but has not been seriously studied (Rao 1969, Wellman 1972).

Species of Meliolales are believed to be host-specific and numerous new species were introduced based on host association (Hansford 1961, Rodríguez Justavino \& Piepenbring 2007). They parasitize only indigenous plants of any particular area, and are limited to a narrow range of host plants that rarely extends to more than one family of phanerogamic hosts (Ciferri 1954, Hansford 1961). In addition, cultures of this group are not obtainable and it is imperative to identify the host, to at least family level, before attempting to identify a new collection (Hansford 1961, Hirata 1972, Saenz \& Taylor 1999, Hongsanan et al. 2015). Previous identifications were mainly based on Hansford (1961), who provided keys to species in Meliolales from various host families, and some researchers only mentioned what Meliolales species are reported from this host family when describing new species, but without citing the source of the information. Hosagoudar \& Archana (2009) provided a checklist of host-fungal association of meliolaceous fungi only from India. On the other hand, online databases, such as Systematic Mycology and Microbiology Laboratory Fungal Database (SMML), sometimes could not provide enough or correct host and fungal information, as well as the references. These makes the taxonomy of Melioales complicated.

In this paper, we aim to provide an updated checklist including current names of host plants with their corresponding Meliolales species, as well as some spelling conflicts and exclusions, for the convenience and unity of future studies.

\section{Materials \& Methods}

\section{Plant information}

Scientific names of host plants were originally obtained from protologes of fungal species or their basionyms and synonyms, and later checked from the online database The Plant List (2013) to obtain the current names and family information. All current names of host plants are listed in Table 1 , with names from protolouge in the bracket.

2. Fungal information

Species accepted in each genus are derived from Index Fungorum (2016) and Mycobank (2016), 
while genera and families accepted in Meliolales are derived from Hongsanan et al. (2015) plus an additional genus, Setameliola described by Reynolds (2010). Only current names in Index Fungorum are listed as accepted names in Table 1. For species that have not been confirmed by Species Fungorum, we listed all possible records to avoid unscientific exclusions, but with the mark "!" at the beginning of each species. Fungal species with "\#” indicate that there are invalid nomenclatures when combined with basionyms, which needs later verification. If epithets in Index Fungorum and Mycobank have no information of year, author or references, they will be treated as invalid names and excluded from Meliolales (Table 2). The spellings of species follow Index Fungorum if they are in conflict with the protologues (Table 3). In cases where two different epithets refer to the same reference, the name that differs from the protologue will be treated as conflicting names in Table 3 .

\section{Results}

The order Meliolales comprises 2403 species (plus 106 uncertain species) distributed in two families and nine genera and are associated with 194 host families, with the preference to Fabaceae. An additional 20 records in Index Fungorum or Mycobank are excluded from the order.

The family Armatellaceae comprises a monotypic genus Armatella (18 species) infecting two host families (Lauraceae and Lamiaceae). The family Meliolaceae comprises eight genera Amazonia (64 species), Appendiculella (75 species), Asteridiella (367 species), Cryptomeliola (3 species), Endomeliola (1 species), Irenopsis (152 species), Meliola (1703 species) and Setameliola (20 species), with 2385 species (plus 106 uncertain species) infecting 194 host families.

\section{Discussion}

The list is sorted by the plant families and genera alphabetically. The genus or family name of a host plant can be searched to check corresponding fungal species occurring on them. Afterwards, morphological comparisons among species that occur on the same host genus or family can be done for determining the identity of the fungal species. For the ecological distribution of a given Meliolales species, the fungal name can be searched in Systematic Mycology and Microbiology Laboratory Fungal Database (SMML) coupled with the list we provide in this study, to gain knowledges of the host range and geographical distribution.

There are a large number of species of Meliolaceae that have been recorded from different plant genera, and members of Armatellaceae seem to be specific to the host family Lauraceae (only 1 exception from Lamiaceae). On the other hand, Meliola thailandicum Hongsanan \& K.D. Hyde has been reported from different host orders, which was confirmed by analysis of LSU and ITS sequence data (Hongsanan et al. 2015). It was the first time that the same Meliolales species occurs on different host orders with molecular evidence.

It is not straight forward to check the host genus or species from protologues when identifying Meliolales species, as the plant name from old literatures may now be a synonym of another species. We have found cases that a specific plant species has been transferred to another genus or even classified under another family (e.g. Myrcia is a plant genus under the family Myricaceae, but the species Myrcia deflexa belongs in the family Myrtaceae). Therefore, we provide the current name for the host species in Table 1. 

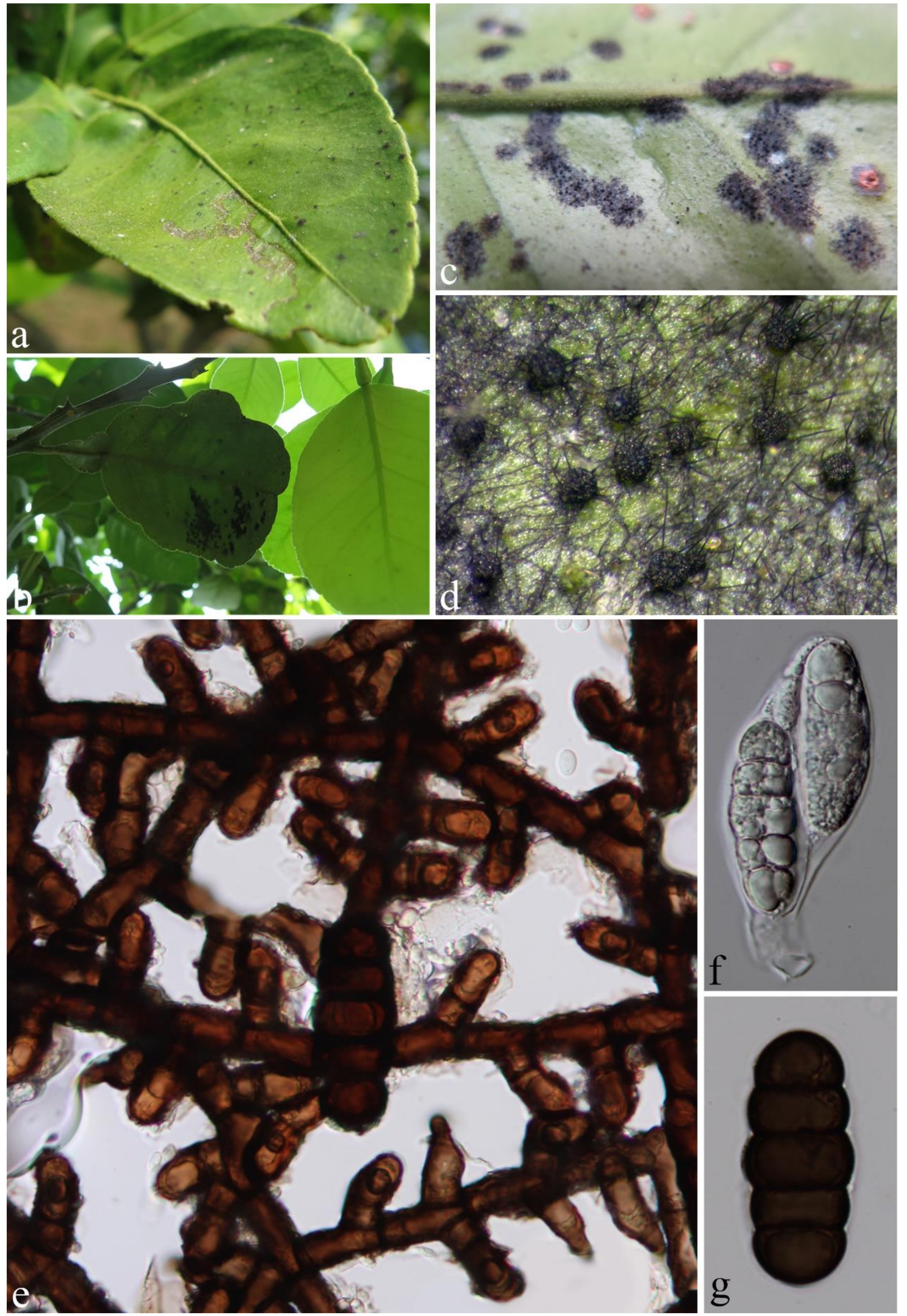

Figure 1 - Meliola citri-maximae on Citrus maxima. a-d Colonies on the leaf surface. e Germinated ascospore with hyphopodia and phialides form on hyphae. f Young ascus. g Mature ascospore. 
Reynolds (2010) introduced a new genus Setameliola based on the presence of both mycelial and perithecial setae, but without molecular data. Hongsanan et al. (2015) only examined generic type specimens of genera accepted by Lumbsch \& Huhndorf (2010), without discussing the newly described genus Setameliola. Nevertheless, we accept this genus as followed in Index Fungorum (2016), but it needs to be further confirmed by molecular analyses.

Table 1 List for identifying Meliolales species based on host associations.

\begin{tabular}{|c|c|c|c|}
\hline Host families & Host species & Fungal species & References \\
\hline \multirow[t]{33}{*}{ Acanthaceae } & - & $\begin{array}{l}\text { Asteridiella } \\
\text { acanthacearum Hosag. } \\
2006\end{array}$ & $\begin{array}{l}\text { Hosagoudar et } \\
\text { al. 2006c }\end{array}$ \\
\hline & - & $\begin{array}{l}\text { Asteridiella poracensis } \\
\text { Petr. } 1959\end{array}$ & Petrak 1958 \\
\hline & - & Meliola culebrensis & Hansford \\
\hline & & Hansf. 1957 & $1957 \mathrm{~b}$ \\
\hline & - & Meliola vanderystii & Hansford \\
\hline & & Hansf. 1957 & $1957 b$ \\
\hline & Avicennia germinans & Asteridiella sepulta (Pat.) & Hansford \\
\hline & (Avicennia nitida) & Hansf. 1961 & 1961 \\
\hline & Barleria strigosa & Meliola barleriae & Srinivasulu \\
\hline & & Srinivas. 1974 & 1974 \\
\hline & Beloperone sp. & $\begin{array}{l}\text { Meliola beloperonis } \\
\text { Viégas } 1944\end{array}$ & Viégas 1944 \\
\hline & Brillantaisia owariensis & Meliola brillantaisiae & Hansford \& \\
\hline & (Brillantaisia nitens) & Hansf. \& Deighton 1948 & $\begin{array}{l}\text { Deighton } \\
1948\end{array}$ \\
\hline & $\begin{array}{l}\text { Brillantaisia owariensis } \\
\text { (Brillantaisia patula) }\end{array}$ & $\begin{array}{l}\text { Asteridiella anastomosans } \\
\text { (G. Winter) Hansf. } 1961\end{array}$ & $\begin{array}{l}\text { Hansford } \\
1961\end{array}$ \\
\hline & $\begin{array}{l}\text { Cynarospermum asperrimum } \\
\text { (Blepharis asperrima) }\end{array}$ & $\begin{array}{l}\text { Meliola blepharidis Thite } \\
\text { \& C.R. Patil } 1985\end{array}$ & Thite \& Patil \\
\hline & Diotacanthus sp. & Asteridiella diotacanthi & Hosagoudar \\
\hline & Graptophyllum pictum & Irenopsis graptophylli & Hansford \\
\hline & Hygrophila costata & $\begin{array}{l}\text { Hanst. } 1954 \\
\text { Asteridiella irregularis }\end{array}$ & $\begin{array}{l}1954 \\
\text { Hansford }\end{array}$ \\
\hline & (Hygrophila brasiliensis) & (F. Stevens) Hansf. 1961 & 1961 \\
\hline & Hypoestes forsskaolii & !Meliola hypöestes E. & Castellani \\
\hline & (Hypoestes paniculata) & Castell. 1947 & 1947 \\
\hline & Hypoestes verticillaris & $\begin{array}{l}\text { Meliola hypöestes Hansf. } \\
1947\end{array}$ & $\begin{array}{l}\text { Hansford } \\
1947 \mathrm{a}\end{array}$ \\
\hline & Justicia ?laxa & $\begin{array}{l}\text { Meliola justiciae Hansf. } \\
1938\end{array}$ & $\begin{array}{l}\text { Hansford } \\
1938\end{array}$ \\
\hline & Justicia flava & Meliola acanthacearum & Hansford \\
\hline & & Hansf. 1946 & 1946 \\
\hline & Justicia sp. & Asteridiella justiciae & Hosagoudar \\
\hline & & $\begin{array}{l}\text { Hosag. \& G. Rajkumar } \\
2004\end{array}$ & $2004 b$ \\
\hline & Mackenziea gracilis* & $\begin{array}{l}\text { Meliola mackenzieae } \\
\text { Hosag. et al. } 2000\end{array}$ & $\begin{array}{l}\text { Hosagoudar et } \\
\text { al. } 2000 \mathrm{~b}\end{array}$ \\
\hline & Mendoncia coccinea ${ }^{*}$ & Asteridiella mendonciae & $\begin{array}{l}\text { Hansford } \\
1961\end{array}$ \\
\hline & Nilgirianthus heyneanus* & Meliola nilgirianthi & Hosagoudar \\
\hline & & Hosag. 1990 & \& Goos 1990a \\
\hline & Phaulopsis imbricata & $\begin{array}{l}\text { Asteridiella phaulopsidis } \\
\text { Hosag. } 2006\end{array}$ & $\begin{array}{l}\text { Hosagoudar } \\
2006\end{array}$ \\
\hline & Ruellia coccinea* & Meliola acanthacearum & Hansford \\
\hline
\end{tabular}


Sclerochiton obtusisepalus

Stenandrium scabrosum*

Strobilanthes amabilis*

Strobilanthes atropurpureus

Strobilanthus sp.

Thunbergia alata

Thunbergia alata

Thunbergia alata

Thunbergia chrysops

Thunbergia cynanchifolia

Achariaceae

Achatocarpaceae

Actinidiaceae

Adoxaceae

Sambucus javanica

Viburnum cylindricum

Hydnocarpus hutchinsonii*

Hydnocarpus pentandrus

Saurauia elegans*

Saurauia elegans* consimilis) sydowii)

Saurauia sp.

?Viburnum sp.
Meliola sclerochitonicola Hansf. 1961

Hansford

1961

Meliola cladacantha Cif. Ciferri 1954

1954

Meliola strobilanthicola Biju et al.

Hosag. et al. 2005

2005

Appendiculella nepalensis Chaudhary et R.K. Chaudhary et al. 1994

Asteridiella

al. 1994

strobilanthicola Hosag. et 2005

al. 2005

Asteridiella thunbergiae Hansford

F. Stevens \& Roldan ex 1962

Hansf. 1963

Meliola alatae Mibey 1997

Mibey \&

Hawksworth

1997

Meliola thunbergiae Hansford

Hansf. 19351938

Asteridiella thunbergiae- Hansford

chrysopis (Hansf. \&

Deighton) Hansf. 1961

Meliola thunbergiicola

Hansf. \& Deighton 1948

1961

Hansford \&

Deighton

1948

Asteridiella deightonii

Hansford

(Hansf.) Hansf. 1961

1961

Irenopsis caloncobae

Hansf. 1946

Hansford

1946

Meliola hydnocarpi

Hansf. 1957

Hansford

$1956 \mathrm{~b}$

Meliola hydnocarpi

Hansford

Hansf. 1957

$1956 b$

Asteridiella

hydnocarpigena Hosag. \& 2013b

C. Jagath Timmaih 2013

Meliola molfinoi Speg.

1924

Spegazzini

1924

Asteridiella papillifera

(Syd. \& P. Syd.) Hansf.

1961

Asteridiella saurauina

Hansford

1961

Hansf. 1957

Hansford

$1956 b$

Meliola notabilis Petr.

1959

Saurauia prainiana (Saurauia

Meliola saurauiae Syd. 1939

Petrak 1958

Saurauia prainiana (Saurauia

Meliola saurauiae Syd.

1939

Sydow \&

Sydow 1939

Sydow \&

Sydow 1939

Asteridiella obscura (F. Hansford

Stevens) Hansf. $1961 \quad 1961$

Meliola yatesii (Syd. \& P. Index

Syd.) Trotter 1926

Fungorum

2016

Meliola sambuci Hansf. Hansford

1954

1954

Meliola simaoensis B. Song et al.

Song \& Goos $2002 \quad$ 2002a 
Viburnum odoratissimum

Viburnum odoratissimum

Viburnum propinquum

Viburnum punctatum

Viburnum sp.

Viburnum tinoides

Altingiaceae

Altingia chinensis

Altingia chinensis

Amaranthaceae Chamissoa altissima

?Anacardiaceae

Anacardiaceae

Anacardium occidentale

Anacardium occidentale

Antrocaryon micraster

Astronium fraxinifolium

Buchanania arborescens

Buchanania cochinchinensis (Buchanania lanzan)

Buchanania cochinchinensis

(Buchanania lanzan)

Buchanania cochinchinensis (Buchanania latifolia)

Buchanania nitida

Comocladia glabra

Comocladia sp.

Dracontomelon dao

Duvaua dependens*

Gluta travancorica

Gluta travancorica

Holigarna grahamii

Lannea coromandelica
Asteridiella viburni (Syd. Hansford \& P. Syd.) Hansf. 1961

Meliola viburnicola Hansford Hansf. 19551955

Asteridiella viburni (Syd. Hansford \& P. Syd.) Hansf. 1961

Meliola goosii Hosag. Hosagoudar 1991

1991a

Meliola yuanjiangensis Jiang 1989

G.Z. Jiang 1989

Meliola aequatoriensis $\quad$ Petrak 1948

Petr. 1948

Appendiculella altingiae Song et al. Y.X. Hu \& B. Song 19961996 a

Meliola altingiae B. Song Song et al. \& Y.X. Hu 1997

1997a

Meliola chamissoae

Hansf. 1955

Hansford

1955

Meliola multiseta Beeli Beeli 1927

1927

Appendiculella anacardii Devi \& T.P. Devi \& Choudhry Choudhry 2005

2005

Meliola anacardii Zimm. Hansford

1902

1949

Meliola geniculata Syd. et Sydow et al. al. 1911

1911

Meliola weigeltii Kunze

1817

Index

Fungorum

2016

Meliola hamata Syd. \& P. Sydow \&

Syd. 1914

Sydow 1914a

Meliola ardigoosii Hosag. Hosagoudar

\& T.K. Abraham 1999 \& Abraham

1999a

Meliola buchananiicola Hosagoudar

Hosag. 1990

\& Goos 1990a

Meliola hui B. Song et al. Song et al.

$2002 \quad 2002 \mathrm{a}$

Meliola buchananiae F. Hansford

Stevens ex Hansf. 1957 1957b

Irenopsis comocladiae (F. Stevens 1927

Stevens) F. Stevens 1927

Irenopsis comocladiae (F. Stevens 1927

Stevens) F. Stevens 1927

Meliola opaca Syd. \& P. Sydow \&

Syd. 1913

Meliola cookeana Speg.

1881

Sydow 1913b

Index

Fungorum

2016

Meliola glutae Hosag. \&

T.K. Abraham 1997

Hosagoudar et

al. 1997

Meliola gluticola Hosag.

\& Sabeena 2011

Hosagoudar

\& Sabeena

2010

Meliola holigarnae F. Stevens 1928

Stevens 1928

Meliola lanneae Hosag. \& Hosagoudar Manojk. 2003

$2003 c$ 
Lannea coromandelica

(Odina wodier)

Lithraea brasiliensis

Lithraea molleoides

Loxostylis alata

Mangifera indica

Mangifera sp.

Mauria simplicifolia

Melanochyla tomentosa*

Nothopegia colebrookiana

Parishia insignis*

Pistacia sp.

Pseudospondias microcarpa

Rhus dentata

Rhus glaucescens*

Rhus laevigata (Rhus incana)

Rhus longispina

Rhus sp.

Rhus tomentosa

Rhus villosa*

Schinus latifolius

Schinus latifolius

Schinus sp.

Schinus sp.

Schinus terebinthifolia

Semecarpus anacardium*

Semecarpus anacardium*

Semecarpus cassuvium*
Meliola geniculata Syd. et Sydow et al.

al. 1911

1911

Meliola lanigera Speg. Spegazzini

$1909 \quad 1909$

Meliola lithraeae Hansf. Hansford

1955

$1953 b$

Meliola loxostylidis

Doidge 1920b

Doidge 1920

Meliola mangiferae Earle Earle 1904

1905

Asteridiella mangiferae Jana et al.

Jana et al. $2005 \quad 2005 a$

Meliola irradians Gaillard Index

1892

Fungorum

2016

Meliola melanochylae

Hansford

Hansf. 1954

1954

Meliola nothopegiae

Hansford

Hansf. 1957

$1956 b$

Meliola parishiae Hosag. Hosagoudar et et al. 2014

al. 2014

!Meliola pistaciae $\mathrm{F}$.

Stevens \& Roldan 1935

Stevens \&

Meliola pseudospondiadis

Roldan 1935

Hansf. \& Deighton 1948

Hansford \&

Deighton

1948

Meliola geniculata Syd. et Sydow et al. al. 1911

1911

Meliola rhois Henn. 1893 Hennings

1893

Meliola dummeri Hansf. Hansford

1938

1938

Meliola rhoina Doidge

Doidge \&

1928

Sydow 1928

Irenopsis kentaniensis

Stevens 1927

(Doidge) F. Stevens 1927

Meliola geniculata Syd. et Sydow et al.

al. $1911 \quad 1911$

Meliola dummeri Hansf. Hansford

1938

1938

Meliola chilensis Speg.

1921

Spegazzini

1921

Meliola rhoina Doidge

Doidge \&

1928

Sydow 1928

Irenopsis brasiliensis

(Speg.) Hansf. 1961

Hansford

1961

Meliola lithraeae Hansf.

1955

Hansford

$1953 b$

Irenopsis schini-

de Macedo et

terebinthifolii D.M.

al. 2010

Macedo \& R.W. Barreto

2011

Meliola abrahamii Hosag. Hosagoudar et et al. 2001

al. 2001c

Meliola semecarpi-

Hosagoudar et anacardii Hosag. et al.

al. $1994 \mathrm{a}$

1994

Meliola pachychaeta Syd. Sydow 1922b

1922 
Semecarpus sp.

Semecarpus sp.

Semecarpus $\mathrm{sp}$.

Semecarpus sp.

Semecarpus travancorica*

Semecarpus travancorica*

Semecarpus travancorica*

Sorindeia juglandifolia

Spondias purpurea

Spondias sp.

?Spondias sp.

Tapirira guianensis

Tapirira sp.

?Tapirira sp.

Thyrsodium $\mathrm{sp}$.

Trichoscypha ?arborea

Ancistrocladacea e

Anisophylleaceae

Ancistrocladus heyneanus

Anisophyllea laurina

Annonaceae
Meliola abraensis Petr. 1959

Meliola semecarpi Syd. \& Sydow 1923a P. Syd. 1923

Meliola semecarpicola Hansford

Hansf. 1958

$1957 \mathrm{c}$

Meliola shivarajui Hosag. Hosagoudar

\& Kamar. 2002

Meliola

padmanabhapurica

Hosag. \& B. Fathima

2013

Meliola sanjappae Hosag. Hosagoudar et et al. 2010

Meliola travancoricae

Hosag. 1991

2002a

Hosagoudar

\& Fathima

2013

Meliola sorindeiae Hansf.

\& Deighton 1948

Amazonia corozalensis

Bat. \& Nascim. 1957

Irenopsis comocladiae (F.

Stevens) F. Stevens 1927

Meliola nicaraguensis

Speg. 1921

Meliola tapiriricola $\mathrm{F}$.

Stevens \& Tehon 1926

Meliola tapirirae F.

Stevens \& Tehon 1926

Amazonia

anacardiacearum $\mathrm{F}$.

Stevens 1927

Meliola cupaniae-majoris

Bat. 1953

Meliola trichoscyphae

Hansf. \& Deighton 1957

Meliola ancistrocladi

Hosag. 1990

Meliola anisophylleae

Hansf. \& Deighton 1948

Asteridiella subglabroides

Hansf. 1957

Meliola varicuspis $\mathrm{F}$.

Stevens \& Tehon 1926

Anaxagorea javanica

Annona purpurea

Annona sp.

Annona sp.

Annona sp.

Artabotrys monteiroae (Artabotrys nitidus)
Asteridiella anaxagoreae

Hosag. et al. 2014

Meliola annonae $\mathrm{F}$.

Stevens 1928

Asteridiella longipoda

(Gaillard) Hansf. 1961

Meliola anonacearum $\mathrm{F}$.

Stevens 1928

Meliola jahnii Toro 1934

Meliola artabotrydis

Hansf. 1938 al. 2010c

Hosagoudar

\& Goos 1991

Hansford \&

Deighton

1948

Batista \&

Maia 1957

Stevens 1927

Spegazzini

1923

Stevens \&

Tehon 1926

Stevens \&

Tehon 1926

Stevens 1927

Index

Fungorum

2016

Hansford

$1956 b$

Hosagoudar

\& Goos 1990a

Hansford \&

Deighton

1948

Hansford

$1956 b$

Stevens \&

Tehon 1926

Hosagoudar et

al. 2014

Stevens 1928

Hansford

1961

Stevens 1928

Chardón \&

Toro 1934

Hansford

1938 
Artabotrys zeylanicus

Cananga odorata

Cleistopholis patens

(Cleistopholis verschuerenii)

Cyathocalyx zeylanicus

Goniothalamus cardiopetalus

Goniothalamus

rhynchantherus

Goniothalamus wightii

Guatteria cubensis

Maasia sumatrana (Polyalthia sumatrana)

Meiogyne pannosa (Unona pannosa)

Meiogyne pannosa (Unona pannosa)

Mitrephora heyneana

Monanthotaxis buchananii

Monodora angolensis

(Monodora gibsonii)

Monodora myristica

Popowia maitlandii*

Popowia maitlandii*

Uvaria angolensis (Uvaria

bukobensis)

Uvaria lucida

Uvaria sp.

Uvaria sp.

Uvaria sp.

Uvaria welwitschii

Xylopia aethiopica

Xylopia aethiopica
Meliola artabotrydicola Hosag. \& T.K. Abraham 1998

Meliola canangae $\mathrm{F}$.

Stevens ex Hansf. 1957

Meliola cleistopholidis

Hansf. 1945

Meliola cyathocalycis

Jana et al. 2005

Meliola

goniothalamigena Hosag.

$\&$ C. Jagath Timmaih

2013

Irenopsis goniothalami Hosagoudar

Hosag. \& T.K. Abraham 1997

\& Abraham

1996

Amazonia goniothalami

Hosag. et al. 2001

Meliola guatteriae Mig.

Rodr. 1999

Meliola polyalthiae

Hansf. 1955

Meliola unonae Hosag. \&

T.K. Abraham 1998

Meliola unonicola Hosag. \& T.K. Abraham 1997

Meliola mitrephorae Hosag. \& Rajendran 1989

Meliola monanthotaxis Mibey 1997

Meliola monodorae

Hansf. 1938

Meliola monodorae

Hansf. 1938

Meliola borneensis Syd.

\& P. Syd. 1923

Meliola maitlandii Hansf.

1946

Hosagoudar et

al. 2001d

Rodríguez \&

Minter 1998

Hansford

1955

Hosagoudar

\& Abraham

$1998 b$

Hosagoudar \& Abraham

1996

Hosagoudar

\& Rajendran

1989

Mibey \&

Hawksworth

1997

Hansford

1938

Hansford

1938

Sydow 1923b

Meliola uvariicola Hansf. Hansford

1945

Meliola mombasana

Mibey 1997

1945a

Mibey \&

Hawksworth 1997

Meliola borneensis Syd. Sydow 1923b \& P. Syd. 1923

Meliola lagunensis Hansf. Hansford 1963

1962

Meliola ngerechiana

Mibey 1997

Mibey \&

Hawksworth

1997

Meliola ramicola Hansf.

1938

Hansford

1938

Meliola kuprensis

Deighton 1958

Deighton

1957

Meliola xylopiae F.

Stevens 1928 
Xylopia aromatica (Xylopia grandiflora)

Xylopia quintasii

Xylopia sericea

Apiaceae

Centella asiatica

Apocynaceae

Aganosma sp.

Allamanda cathartica

Alstonia congensis

Alstonia congensis

Alstonia lenormandii

(Alstonia comptonii)

Alstonia scholaris

Alstonia scholaris

Alstonia scholaris

Alyxia concatenata (Paralstonia clusiaceae)

Alyxia leucogyne

Alyxia stellata

Alyxia stellata (Alyxia oliviformis)

Alyxia stellata (Alyxia oliviformis)

Anodendron affine

Anodendron paniculatum

Asclepias sp.

Asclepias sp.
Meliola xylopiae $\mathrm{F}$.

Stevens 1928

Stevens 1928

Meliola golaensis Deighton

Deighton $1958 \quad 1957$

Meliola xylopiae-sericiae Pinho et al.

D.B. Pinho \& O.L. 2013

Pereira 2013

Meliola centellae Pinho \& Pinho et al.

O.L. Pereira $2012 \quad 2012$

Meliola clavatispora Spegazzini

Speg. 1889

Meliola intermedia

1889

Gaillard 1892

Meliola reflexa Hansf. Hansford

1941

1941

Meliola

thiruvananthapurica

Hosag. \& T.K. Abraham

1999

Meliola amboinensis Syd. Sydow 1922b

1922

Asteridiella allamandae

Jana \& S.N. Ghosh 2008

Meliola alstoniicola

Hansf. 1945

Meliola hendrickxii

Hansf. 1946

Hosagoudar

\& Abraham

$1999 b$

Meliola alstoniae-

comptonii B. Huguenin

1969

Meliola alstoniae Koord.

1907

Meliola dehradunensis

Gawande \& D.K.

Agarwal 2004

Meliola peringamalensis

Hosag. \& Kamar. 2006

Meliola laevigata Syd. \&

P. Syd. 1912

Meliola alyxiicola B.

Huguenin 1969

Meliola moerenhoutiana

Mont. 1842

Amazonia alyxiae Hansf.

1957

Meliola alyxiae F.

Stevens 1925

Meliola anodendri

Sawada \& W. Yamam.

1959

Meliola anodendricola

Hosag. 2006

Meliola

asclepiadacearum Hansf.

1945

Meliola buxaduarii Jana

\& S.N. Ghosh 2008
Jana \& Ghosh

2008c

Hansford

$1945 b$

Hansford

1946

Index

Fungorum

2016

Koorders

1907

Gawande et

al. 2004

Hosagoudar

2006

Sydow \&

Sydow 1912b

Index

Fungorum

2016

Montagne

1842

Hansford

$1957 b$

Stevens 1925

Stevens 1925

Hosagoudar

\& Biju 2006

Hansford

$1945 \mathrm{a}$

Jana \& Ghosh 2008e 
Aspidosperma polyneuron

Aspidosperma quebrachoblanco (Aspidosperma quebracho)

Aspidosperma quebrachoblanco (Aspidosperma quebracho)

Baissea zygodioides (Baissea aframensis)

Carissa bispinosa (Carissa arduina)

Carissa carandas

Carissa carandas

Carissa carandas

Carissa spinarum

Ceropegia sp.

Chilocarpus denudatus

(Chilocarpus atroviridis)

Cynanchum abyssinicum

(var. tomentosum)

Cynanchum sp.

Dischidia sp.

Echites angustifolia

Funtumia africana

Funtumia elastica

Funtumia sp.

Gonioma kamassi

Gymnema sp.

Hancornia speciosa

Hemidesmus indicus

Hemidesmus indicus

Hemidesmus indicus

Hemidesmus indicus

Holarrhena floribunda

(Holarrhena africana)
Asteridiella aspidospermatis (Speg.)

Hansf. 1961

Meliola laevipoda Speg. 1891

Hansford

1961

Spegazzini

1891a

Meliola membranacea

Starbäck 1899

Starbäck 1899

Meliola baisseae

Deighton

Deighton 1958

1957

Meliola carissae Doidge 1922

Meliola capensis

Doidge 1922

(Kalchbr. \& Cooke)

Theiss. 1912

Meliola carissae Doidge Doidge 1922

1922

Meliola integripoda

Hosag. et al. 1998

Hosagoudar et

Meliola carissae Doidge 1922

Meliola ceropegia Hosag.

\& V.S. Ramach. 1991

Meliola chilocarpi Hosag.

\& T.K. Abraham 1998

Meliola

asclepiadacearum Hansf.

1945

Meliola cynanchi Hosag.

et al. 2006

Meliola odontochaeta

Syd. 1922

Meliola echitis Hansf.

1957

Meliola voacangae Hansf.

\& F. Stevens 1937

Meliola funtumiae Beeli

1920

Meliola funtumiae Beeli

1920

Amazonia goniomae

Doidge 1924

Meliola gymnemae Jana

et al. 2005

Meliola hancorniae J.L.

Bezerra \& T.T. Barros

1970

!Meliola hemidesmi Jana

\& S.N. Ghosh 2008

Meliola hemidesmi Kamal

\& B.K. Gupta 1987

Meliola hemidesmicola

Hosag. 1996

Meliola simillima Ellis \&

Everh. 1898

Meliola holarrhenicola

Deighton 1958 al. 1998 a

Doidge 1922

Theissen 1912

Hosagoudar \& Goos 1991

Hosagoudar et al. $1998 d$ Hansford $1945 \mathrm{a}$

Hosagoudar et al. $2006 \mathrm{~b}$ Sydow 1922b

Hansford $1956 b$

Hansford

1937

Beeli 1920

Beeli 1920

Index

Fungorum

2016

Jana et al. $2005 \mathrm{e}$

Index

Fungorum 2016

Jana \& Ghosh 2008d

Kamal \& Gupta 1986 Hosagoudar 1996

Index Fungorum 2016 Deighton 1957 
Holarrhena pubescens

Holarrhena pubescens

(Holarrhena antidysenterica)

Holarrhena pubescens

(Holarrhena antidysenterica)

Hoya luzonensis\#

Hunteria zeylanica (Hunteria corymbosa var. roxburghiana)

Ichnocarpus frutescens

Ichnocarpus frutescens

Ichnocarpus frutescens

(Ichnocarpus volubilis)

Ichnocarpus frutescens

(Ichnocarpus volubilis)

Ichnocarpus frutescens

(Ichnocarpus volubilis)

Kamettia caryophyllata

Kamettia caryophyllata

(Ellertonia rheedei)

Landolphia buchananii

(Landolphia ugandensis)

Landolphia dulcis

(Carpodinus dulcis)

Landolphia kirkii

Landolphia sp.

Malouetia guatemalensis (Malouetia panamensis)

Malouetia heudelotii

Mandevilla sp.

Mandevilla torosa (Echites brownei)

Melodinus australis

Motandra sp.

Ochrosia sp.

Odontadenia nitida

Oncinotis tenuiloba

(Oncinotis inandensis)

Parsonsia alboflavescens
Meliola holarrhenae-

pubescens Jana et al. 2005

Meliola holarrhenae

Jana et al.

Hansf. \& Thirum. 1948

Hansford \&

Thirumalacha

r 1948

Meliola simillima Ellis \& Index

Everh. 1898

Fungorum

2016

Meliola hoyae Sacc. 1919 Saccardo

1917

Meliola hunteriae Hosag. Hosagoudar 1990

Meliola frutescentis

Hosag. et al. 1999

\& Goos 1990a

Hosagoudar et

al. $1999 \mathrm{~b}$

Meliola ichnocarpicola

Hansf. 1961

Hansford

1961

!Meliola ichnocarpi $\mathrm{F}$.

Stevens \& Roldan 1935

Stevens \&

Roldan 1935

Meliola ichnocarpi Hansf. Hansford \&

\& Thirum. 1948

Thirumalacha

r 1948

Meliola ichnocarpi-

volubili Hansf. 1963

Hansford

1962

Meliola kamettiae Hosag. Hosagoudar

\& Riju 2010

\& Riju 2010

Meliola ellertoniae

Hosagoudar

Hosag. \& T.K. Abraham

1998

\& Abraham

$1998 b$

Meliola landolphiicola

Hansf. 1945

Hansford

1945a

Meliola voacangae Hansf. Hansford

\& F. Stevens $1937 \quad 1937$

Meliola keniensis Mibey Mibey \& 1997

Hawksworth

1997

Meliola landolphiae Hansford

Hansf. $1937 \quad 1937$

Meliola wardii F. Stevens Stevens 1928

1928

Meliola malouetiae Hansford \&

Hansf. \& Deighton 1948 Deighton

1948

Meliola mandevillae F. Stevens 1928

Stevens 1928

Meliola simillima Ellis \& Index

Everh. 1898

Meliola melodini Hansf. Hansford

1953

1953a

Meliola motandrae Hansf. Hansford 1941

1941

Meliola guamensis Syd. $\quad$ Sydow 1921

\& P. Syd. 1921

Meliola

tabernaemontanae Speg. 1912

1912

Meliola oncinotidis

Doidge 1948

Meliola parsonsiicola

Hosag. 2006
Spegazzini

Doidge 1948

Hosagoudar et al. 2006c 
Parsonsia straminea

Periploca linearifolia

Pinochia corymbosa

(Forsteronia corymbosa)

Pleioceras barteri

Plumeria rubra

Rauvolfia mombasiana

Rauvolfia nitida

Saba comorensis (Landolphia florida)

Secamone platystigma*

Serenoa repens (Sabal serrulata)

Strophanthus divaricatus

Strophanthus sp.

Strophanthus speciosus

Strophanthus wightianus

Tabernaemontana africana (Conopharyngia longiflora)

Tabernaemontana alternifolia (Ervatamia heyneana)

Tabernaemontana alternifolia (Tabernaemontana heyneana)

Tabernaemontana citrifolia

Tabernaemontana citrifolia

Tabernaemontana hystrix

Tabernaemontana longipes

Tabernaemontana sp.

Tabernaemontana sp.

Tabernaemontana sp.

Tabernaemontana sp.

Tabernaemontana sp. (Conopharyngia sp.)

Telosma africana
Meliola carissae Doidge 1922

Meliola periplocae Mibey Mibey \& 1999

Kokwaro

1999

Meliola forsteroniae (F. Hansford Stevens) Hansf. $1949 \quad 1949$

Meliola pleioceratis Hansford

Hansf. 1957

$1956 b$

Asteridiella plumeriae Hansford (Hansf. \& Deighton) 1961

Hansf. 1961

Meliola rauvolfiae Mibey 1997

Mibey \&

Hawksworth 1997

Meliola euopla Syd. 1928 Stevens 1928

Meliola landolphiae- Hansford floridae Hansf. $1938 \quad 1938$

Meliola secamones Hansf. Hansford 1944

1944

Meliola palmicola $\mathrm{G}$. Winter 1887

Winter 1887

Amazonia rogergoosii B. Song et al.

Song et al. 2002 2002b

Meliola strophanthicola Hansford

Hansf. 1961

1961

Asteridiella strophanthi Hansford

(Doidge) Hansf. 1961

Meliola strophanthicola Hansford

Hansf. 1961

1961

Meliola voacangae Hansf. Hansford \& F. Stevens $1937 \quad 1937$

Meliola ervatamiae Hosagoudar

Hosag. $1988 \quad 1987 \mathrm{a}$

Meliola pepparaensis Hosagoudar

Hosag. \& T.K. Abraham \& Abraham

1998

$1998 b$

\#Irene escharoides Syd. $\quad$ Ciferri 1954

1926

Meliola isothea Syd. 1926 Sydow 1926

Meliola tabernaemontanae Speg.

1912

\#Irene escharoides Syd. Sydow 1926

1926

Irenopsis andamanica Hosagoudar et

Hosag. et al. 2014

al. 2014

Meliola beebei F. Stevens

1928

Meliola

tabernaemontanicola

Hansf. \& Thirum. 1948

Meliola wardii F. Stevens

1928

Meliola

tabernaemontanae Speg.

1912

Meliola hughesiana

Hansf. 1957
Hansford \&

Thirumalacha r 1948

Stevens 1928

Spegazzini

1912

Hansford

$1956 \mathrm{~b}$
Stevens 1928 
Thevetia neriifolia

Toxocarpus beddomei*

Trachelospermum sp.

Tylophora flexuosa

(Tylophora tenuis)

Tylophora flexuosa

(Tylophora tenuis)

Tylophora indica

Tylophora perrottetiana*

Tylophora sp.

Urceola brachysepala

(Urceola imberbis)

Urceola javanica

Vallaris sp.

Vinca sp.

Vincetoxicum capparidifolium (Tylophora capparidifolia)

Voacanga africana

Voacanga foetida

Voacanga $\mathrm{sp}$.

Voacanga sp.

Voacanga thouarsii

(Voacanga obtusa)

Willughbeia cordifolia\#

Willughbeia sp.

Wrightia coccinea

Wrightia pubescens subsp. laniti (Wrightia laniti)

Wrightia tinctoria

Aquifoliaceae Ilex dioica (Ilex nitida)

Ilex formosana

Ilex malabarica
Meliola modesta Syd. 1926

Meliola toxocarpi Hosag. Hosagoudar \& V.T. Antony 1988

\& Antony

1988

Meliola trachelospermi Yates 1918

H.S. Yates 1918

Meliola dimorphochaeta Patil \&

M.S. Patil \& Maham. Mahamulkar 1999

1999

Meliola telosmae Rehm

1955

Meliola tylophorae-

indicae Hosag. \&

Manojk. 2004

Meliola telosmae Rehm

1955

Meliola perpusilla Syd. \&

P. Syd. 1913

Meliola depressula Syd.

\& P. Syd. 1917

Meliola urceolae Hansf.

1954

Meliola vallaridis Hosag.

et al. 2007

Meliola amphitricha Fr.

1828

Meliola tylophorae

Hosag. 1990

Meliola voacangicola

Hansf. 1958

Meliola voacangae-

foetidae Hansf. 1954

Asteridiella voacangina

Hansf. 1957

Hansford

1955

Hosagoudar

2004 b

Hansford

1955

Sydow \&

Sydow 1914b

Sydow \&

Sydow 1917a

Hansford

1954

Hosagoudar et al. $2007 \mathrm{~d}$

Index

Fungorum

2016

Hosagoudar

\& Goos 1990a

Hansford

$1957 \mathrm{c}$

Hansford

1954

Hansford

$1957 b$

Meliola voacangae Hansf. Hansford

\& F. Stevens 1937

1937

Asteridiella voacangae

Deighton 1958

Deighton

1957

Appendiculella sororcula

(Speg.) Hansf. 1961

Hansford

1961

Toro 1925

compositarum (Earle)

Toro 1925

Asteridiella wrightiae

Jana et al. 2005

Jana et al.

$2005 b$

Meliola wrightiae H.S. Yates 1918

Yates 1918

Meliola

tabernaemontanae Speg.

1912

Irenopsis maricaensis $(\mathrm{F}$.

Stevens) F. Stevens 1927

Meliola ilicicola W.

Yamam. 1940

Spegazzini

1912

Meliola ilicis-

malabaricae Hosag. \&

Raghu 1993
Stevens 1927

Yamamoto

1940

Hosagoudar

\& Raghu

1993 
Ilex mitis

Ilex montana

Ilex paraguayensis*

Ilex scopulorum

Ilex $\mathrm{sp}$.

Araceae

Araliaceae

Aralia decaisneana

Aralia sp.

Aralia sp.

Aralia sp.

Brassaiopsis sp.

Cheirodendron trigynum

(Cheirodendron gaudichaudii)

Cussonia sp.

Dendropanax arboreus

Eleutherococcus trifoliatus

(Acanthopanax trifoliatus)

Fatsia oligocarpella

Hedera helix

Hedera helix
Meliola knysnae Doidge 1948

Meliola ciferrii Hansf. Hansford

1961

Irenopsis yerbae (Speg.)

Hansf. 1961

\#Meliola lagerheimii

Gaillard 1891

Appendiculella wuyiensis

Y.X. Hu \& B. Song 1996

Meliola alocasiae Syd.

1925

Meliola boninensis Speg. Spegazzini 1921

Asteridiella colocasiae

Hosag. et al. 2014

\#Irenina aracearum $\mathrm{F}$.

Stevens 1927

\#Irenina aracearum $\mathrm{F}$.

Stevens 1927

Meliola dieffenbachiae F. Stevens 1916

Stevens 1916

Appendiculella monsterae

Rodr. Just. 2014

Meliola philodendri F.

Stevens 1916

Meliola philodendricola

Hansf. 1955

Asteridiella pothodis

(Hansf. \& Thirum.)

Hansf. 1961

Meliola araliicola W.

Yamam. 1941

Appendiculella araliae

(Spreng.) Hansf. 1961

Meliola abdulkalamii

Hosag. \& Riju 2011

Meliola araliae Mont.

1842

Meliola brassaiopsidis

Hosag. 1996

Asteridiella cheirodendri

(F. Stevens) Hansf. 1961

Meliola leptidea Syd. \&

P. Syd. 1912

Meliola didymopanacis

Henn. 1895

Meliola acanthopanacis

W. Yamam. 1941

Meliola fatsiae Katum. \&

Y. Harada 1979

Meliola kusanoi Henn.

1900

Meliola payakii Hosag.

1996
Rodríguez

Justavino et

al. 2015

Stevens 1916

Hansford

1955

Hansford

1961

Index

Fungorum

2016

Hansford

1961

Hosagoudar

\& Riju 2011a

Index

Fungorum

2016

Hosagoudar

1996

Hansford

1961

Sydow \&

Sydow 1912a

Hennings

1895a

Index

Fungorum

2016

Katumoto \&

Harada 1979

Hennings

1900a

Hosagoudar

1996 





Bactris sp.

Calamus deerratus

Caryota urens

Coccothrinax argentea

Cocos nucifera

Copernicia augularis*

Copernicia curtissii

(Copernicia pauciflora)

Desmoncus orthacanthos

(Desmoncus major)

Elaeis guineensis (Elaeis

melanococca)

Laccosperma secundiflorum

(Ancistrophyllum

secundiflorum)

Livistona boninensis

(Livistona chinensis var.

boninensis)

Livistona $\mathrm{sp}$.

Mauritia sp.

Palma sp.*

Palma sp.*

Palma sp.*

Phoenix dactylifera

Phoenix reclinata

Prestoea acuminata var. montana (Acrista monticola)

Raphia palma-pinus (Raphia gracilis)

Roystonea regia (Oreodoxa regia)

Aristolochiaceae Aristolochia dorsivenia\#

Aristolochia littoralis

(Aristolochia elegans)

Aristolochia maxima

Aristolochia sp.

Aristolochia tagala

Aristolochia triangularis
Meliola decora Syd. 1939 Sydow \&

Sydow 1939

Meliola calami Hansf. \& Hansford \&

Deighton 1948

Deighton

1948

Meliola caryotae Srinivasulu

Srinivas. $1974 \quad 1974$

Meliola acristae Hansf. Hansford

$1957 \quad$ 1957b

Meliola acristae Hansf. Hansford

$1957 \quad 1957 b$

Meliola palmicola $\mathrm{G}$. Winter 1887

Winter 1887

Meliola sparsipoda Hansford

Hansf. $1955 \quad 1955$

Meliola desmonci Hansf. Hansford $1957 \quad 1956$ b

Meliola melanococcae F. Stevens 1928

Stevens 1928

Meliola mauritiae F. Stevens 1928

Stevens 1928

Meliola livistonae H.S. Yates 1917

Yates 1917

Meliola livistonae H.S. Yates 1917

Yates 1917

Meliola mauritiae F.

Stevens 1928

!Meliola palmarum Index

Kunze \& Fr.

Fungorum

2016

Meliola morrowii $\mathrm{F}$.

Stevens 1928

Stevens 1928

Meliola sparsipoda Hansford

Hansf. 19551955

Meliola palmicola $\mathrm{G}$. Winter 1887

Winter 1887

Meliola palmicola $\mathrm{G}$. Winter 1887

Winter 1887

Meliola acristae Hansf. Hansford 1957

$1957 b$

Meliola mauritiae F. Stevens 1928

Stevens 1928

Meliola regiae Hansf. Hansford

1955

Meliola entebbeensis

Hansf. 1944

1955

Hansford

1944

Meliola aristolochiae F. Stevens \&

Stevens \& Tehon 1926 Tehon 1926

Meliola aristolochiicola Stevens 1928

F. Stevens 1928

Irenopsis aristolochiella Hansford

Hansf. $1958 \quad 1957 \mathrm{c}$

Meliola aristolochigena Hosagoudar

Hosag. \& Archana 2009 \& Archana

2009

Hansford

Meliola catherinensis

Hansf. 1955
1955 


\begin{tabular}{|c|c|c|c|}
\hline \multirow[t]{5}{*}{ Asclepiadaceae } & - & Meliola congoensis & Hansford \\
\hline & & (Beeli) Hansf. 1961 & 1961 \\
\hline & - & Meliola perpusilla Syd. \& & Sydow \& \\
\hline & & P. Syd. 1913 & Sydow 1914b \\
\hline & - & $\begin{array}{l}\text { Meliola telosmae Rehm } \\
1955\end{array}$ & $\begin{array}{l}\text { Hansford } \\
1955\end{array}$ \\
\hline \multirow[t]{19}{*}{ Asparagaceae } & Agave shaferi & Meliola agavicola Mig. & Rodríguez \\
\hline & & Rodr. \& Camino 1987 & $\begin{array}{l}\text { Hernández \& } \\
\text { Camino }\end{array}$ \\
\hline & & & Vilaró 1987 \\
\hline & Behnia reticulata & $\begin{array}{l}\text { Meliola behniae Syd. } \\
1928\end{array}$ & $\begin{array}{l}\text { Doidge \& } \\
\text { Sydow } 1928\end{array}$ \\
\hline & Dracaena aurea & $\begin{array}{l}\text { Meliola dracaenae } \mathrm{F} . \\
\text { Stevens } 1925\end{array}$ & Stevens 1925 \\
\hline & Dracaena fragrans & Meliola dracaenicola & Patouillard \& \\
\hline & & Har. \& Pat. 1908 & Hariot 1908 \\
\hline & Dracaena mannii & $\begin{array}{l}\text { Meliola interrupta Hansf. } \\
\text { \& Deighton } 1948\end{array}$ & $\begin{array}{l}\text { Hansford \& } \\
\text { Deighton } \\
1948\end{array}$ \\
\hline & Dracaena mannii (Dracaena & Meliola shimbaensis & Mibey \& \\
\hline & usambarensis) & Mibey 1997 & $\begin{array}{l}\text { Hawksworth } \\
1997\end{array}$ \\
\hline & Dracaena sp. & $\begin{array}{l}\text { Meliola subdentata Pat. } \\
1897\end{array}$ & $\begin{array}{l}\text { Patouillard } \\
1897\end{array}$ \\
\hline & Dracaena terniflora & $\begin{array}{l}\text { Meliola dracaenae- } \\
\text { terniflorae Hosag. } 1996\end{array}$ & $\begin{array}{l}\text { Hosagoudar } \\
1996\end{array}$ \\
\hline & Lomandra sp. & Meliola lomandrae Hansf. & Hansford \\
\hline & Sansevieria dawei & Meliola sansevieriae & $\begin{array}{l}1953 \mathrm{a} \\
\text { Index }\end{array}$ \\
\hline & & Wakef. 1937 & Fungorum \\
\hline & Sansevieria liberica & Meliola subdentata Pat. & Patouillard \\
\hline & & 1897 & 1897 \\
\hline & Sansevieria trifasciata & !Meliola sansevieriae $\mathrm{R}$. & Index \\
\hline & (Sansevieria laurentii) & Cass. \& Massenot 1966 & $\begin{array}{l}\text { Fungorum } \\
2016\end{array}$ \\
\hline \multirow[t]{2}{*}{ Aspleniaceae } & Asplenium cristatum & Irenopsis pteridicola $(\mathrm{F}$. & Hansford \\
\hline & & Stevens) Hansf. 1955 & 1955 \\
\hline \multirow[t]{11}{*}{ Asteraceae } & - & $\begin{array}{l}\text { Asteridiella negeri } \\
\text { (Hansf) Hansf } 1961\end{array}$ & $\begin{array}{l}\text { Hansford } \\
1961\end{array}$ \\
\hline & - & Meliola spegazziniana $\mathrm{G}$. & Index \\
\hline & & Winter 1888 & $\begin{array}{l}\text { Fungorum } \\
2016\end{array}$ \\
\hline & $\begin{array}{l}\text { Austroeupatorium } \\
\text { inulaefolium (Eupatorium } \\
\text { inulaefolium) }\end{array}$ & $\begin{array}{l}\text { Appendiculella sororcula } \\
\text { (Speg.) Hansf. } 1961\end{array}$ & $\begin{array}{l}\text { Hansford } \\
1961\end{array}$ \\
\hline & Baccharis pingraea & $\begin{array}{l}\text { Appendiculella sororcula } \\
\text { (Speg.) Hansf. } 1961\end{array}$ & $\begin{array}{l}\text { Hansford } \\
1961\end{array}$ \\
\hline & Baccharis rhexioides & $\begin{array}{l}\text { \#Irenina angustispora F. } \\
\text { Stevens \& Roldan } 1935\end{array}$ & Ciferri 1954 \\
\hline & Baccharis rhexioides & $\begin{array}{l}\text { Meliola angustispora } \mathrm{F} . \\
\text { Stevens } 1928\end{array}$ & Stevens 1928 \\
\hline & Baccharis sp. & $\begin{array}{l}\text { Asteridiella abnormis } \\
\text { (Theiss.) Hansf. } 1961\end{array}$ & $\begin{array}{l}\text { Hansford } \\
1961\end{array}$ \\
\hline & Baccharis sp. & $\begin{array}{l}\text { Meliola baccharidis Berk. } \\
\text { \& Ravenel } 1876\end{array}$ & $\begin{array}{l}\text { Berkeley } \\
1876\end{array}$ \\
\hline & Bidens aristosa (Coreopsis & Meliola coreopsidis Thite & Index \\
\hline & aristosa) & \& U.K. Kulk. 1978 & $\begin{array}{l}\text { Fungorum } \\
2016\end{array}$ \\
\hline
\end{tabular}


Bidens sp.

Blumea megacephala

Calea jamaicensis (Calea

pittieri)

Calea solidaginea

Chromolaena glaberrima

(Eupatorium oerstedianum)

Chromolaena maximilianii

(Eupatorium maximiliani)

Chromolaena odorata

(Eupatorium conyzoides)

Chromolaena odorata

(Eupatorium odoratum)

Chromolaena odorata

(Eupatorium odoratum)

Chromolaena sinuata

(Eupatorium sinuatum)

Condylidium iresinoides

(Eupatorium iresinoides)

Critonia morifolia

(Eupatorium morifolium)

Critonia portoricensis

(Eupatorium portoricense)

Elephantopus sp.

Eupatorium sp.

?Gymnopsis sp.

Hymenostephium

microcephalum (Gymnolomia

patens var. macrophylla)

Koanophyllon dolicholepis

(Eupatorium dolicholepis)

Liabum sp.

Mikania nigricans

(Willoughbya firmula)

Mikania scandens

Mikania sp.

Mutisia speciosa

Neurolaena lobata

Olearia argyrophylla

Osmia sp.*

Piper umbellatum

(Pothomorphe umbellata)

Piptocarpha axillaris

Pseudelephantopus spicatus
Appendiculella sororcula (Speg.) Hansf. 1961

Hansford

1961

!Asteridiella blumeicola $\mathrm{Hu} 1990 \mathrm{~b}$

H. $\mathrm{Hu} 1990$

Appendiculella sororcula Hansford

(Speg.) Hansf. 1961

Appendiculella sororcula

(Speg.) Hansf. 1961

Appendiculella sororcula

(Speg.) Hansf. 1961

Appendiculella eupatorii

Meir. Silva et al. 2012

Appendiculella sororcula

(Speg.) Hansf. 1961

!Meliola eupatorii Jana \&

S.N. Ghosh 2008

1961

Hansford

1961

Hansford

1961

Pinho et al.

2012

Hansford

1961

Jana \& Ghosh

$2008 b$

Appendiculella sororcula

(Speg.) Hansf. 1961

Meliola eupatorii

Schmied. 1989

Appendiculella sororcula

(Speg.) Hansf. 1961

Appendiculella sororcula

(Speg.) Hansf. 1961

Appendiculella sororcula

(Speg.) Hansf. 1961

Meliola elephantopi

Hansf. 1958

Appendiculella sororcula

(Speg.) Hansf. 1961

Meliola sordidula (Lév.)

Berl. \& Sacc. 1886

Meliola gymnoloniae

Toro 1934

Appendiculella sororcula (Speg.) Hansf. 1961

Appendiculella sororcula

(Speg.) Hansf. 1961

Meliola willoughbyae

Zimm. 1901

Hansford

1961

Schmiedeknec

ht 1989

Hansford

1961

Hansford

1961

Hansford

1961

Hansford

$1957 \mathrm{c}$

Hansford

1961

Berlese \&

Voglino 1886

Chardón \&

Toro 1934

Hansford

1961

Hansford

1961

Zimmermann

1901

Irenopsis mikaniae Hansf. Hansford \&

\& Deighton 1948

Deighton

1948

Meliola mikaniae Gaillard Gaillard 1892

1892

Meliola mutisiae Firmino

et al. 2012

Pinho et al.

2012

Asteridiella cyclopoda (F.

Stevens) Hansf. 1961

Hansford

1961

Hansford

1946

1946

Appendiculella sororcula

(Speg.) Hansf. 1961

Hansford

1961

Irenopsis tortuosa $(\mathrm{G}$.

Winter) F. Stevens 1927

Irenopsis piptocarphae

Hansf. 1957

Stevens 1927

Asteridiella cyclopoda (F. Stevens) Hansf. 1961
Hansford

$1956 b$

Hansford

1961 
Schistocarpha sp.

Senecio scandens

Senecio sp.

Vernonia arborea

Vernonia sp.

Willoughbya sp.

Atherospermatac eae

Daphnandra micrantha

Doryphora sassafras

Doryphora sassafras

Balsaminaceae

Impatiens capensis

Impatiens sp.

Begoniaceae

Bignoniaceae
Begonia meyeri-johannis

Appendiculella sororcula (Speg.) Hansf. 1961

!Asteridiella blumeicola

H. $\mathrm{Hu} 1990$

Irenopsis senecionis

Hansf. 1961

Asteridiella cyclopoda (F. Stevens) Hansf. 1961

Appendiculella sororcula (Speg.) Hansf. 1961

Appendiculella sororcula (Speg.) Hansf. 1961

Asteridiella daphnandrae

(Hansf.) Hansf. 1961

Appendiculella kiraiensis

(W. Yamam.) Hansf.

1961

Meliola doryphorae

Hansf. 1956

Meliola impatientis

Doidge 1941

Meliola impatientis

Doidge 1941

Meliola begoniae Hansf. Hansford 1947

Asteridiella arachnoidea

(Speg.) Hansf. 1961

Asteridiella peruviana

(Syd. \& P. Syd.) Hansf.

1961

Irenopsis bignoniacearum Stevens 1927

F. Stevens 1927

Irenopsis brasiliensis Hansford

(Speg.) Hansf. 1961

Irenopsis shropshiriana

(F. Stevens) Hansf. 1961

Meliola ariquemensis Bat.

\& Cavalc. 1965

Meliola asperipoda

Hansf. 1955

Meliola hariotii Speg.

1891

Meliola mandingensis

Hansf. 1957

Meliola rockstonensis

Hansf. 1957

Meliola standleyi Hansf.

1955

Meliola thaxteri Hansf.

1961

Meliola tumor F. Stevens

1928

Adenocalymma bracteosum (Spathodea bracteosa) Amphilophium lactiflorum (Distictis lactiflora) Amphilophium paniculatum (Amphilophium vauthieri)
Meliola bignoniacearum

Meliola distictidis Hansf. Hansford 1948

Asteridiella amphilophis (Hansf.) Hansf. 1961

1949

Hansford

1961
F. Stevens 1928
1961

Hansford

1961

Hansford

Farr \&

Rossman

2016

Hansford

1961

sford

Hansford

1961

Hansford

ansford

Hansford

1961

Hansford

1956a

Doidge 1941

Doidge 1941

1947a

Hansford

1961

Hansford

1961

Batista et al

1965a

Hansford

1955

Spegazzini

1891a

Hansford

1957b

Hansford

$1957 b$

Hansford

1955

Hansford

1961

Stevens 1928

Stevens 1928 
Amphilophium sp.

Arrabidaea sp.

Arrabidaea sp.

Bignonia aequinoctialis

(Cydista aequinoctialis)

Bignonia corymbosa

(Phryganocydia corymbosa)

Bignonia sp.

Crescentia cujete

Crescentia cujete

Crescentia sp.

Dolichandra unguis-cati

(Bignonia unguis-cati)

Fridericia nicotianiflora (Arrabidaea nicotianiflora)

Handroanthus heptaphyllus

(Tabebuia ipe)

Jacaranda sp.

Jacaranda $\mathrm{sp.}$

Kigelia africana subsp.

Moosa (Kigelia moosa)

Lundia corymbifera (Lundia umbrosa)

Manaosella cordifolia

(Manaosella platidactyla)

Markhamia lutea (Markhamia

platycalyx)

Markhamia lutea (Markhamia

platycalyx)

Markhamia sp.

Newbouldia laevis

Stereospermum tetragonum

(Stereospermum colais)

Tabebuia ?stenocalycis

Tabebuia berteroi

Tabebuia heterophylla

(Tecoma pentaphylla)

Tabebuia lepidophylla

Tabebuia polyantha

Tabebuia sp.
Asteridiella plebeja

(Speg.) Hansf. 1961

Meliola arrabidaeae

Hansford

Hansf. 1957

Meliola bignoniacearum

F. Stevens 1928

Meliola cydistae F.

Stevens 1928

Meliola bignoniacearum

F. Stevens 1928

Meliola bidentata Cooke

1882

Asteridiella crescentiicola

Hansf. 1957

Meliola crescentiae F.

Stevens 1928

Meliola crescentiae F.

Stevens 1928

Meliola herteri Hansf.

1955

Meliola dentifera Syd. \&

P. Syd. 1916

Asteridiella tabebuiae

(Bat. \& J. Silva) Hansf.

1961

Meliola caput-medusae

Cif. 1933

Meliola gnathonella $\mathrm{F}$.

Stevens \& Tehon 1926

Meliola kigeliae Hansf.

1955

Meliola lundiae F.

Stevens 1928

Meliola manoasellae

Hansf. 1957

Meliola kampalensis

Hansf. 1937

Meliola markhamiae

Hansf. \& F. Stevens 1937

Meliola lanceolato-setosa

Syd. \& P. Syd. 1914

Meliola newbouldiae

Hansf. \& Deighton 1948

Asteridiella schlegeliae

(F. Stevens) Hansf. 1961

Meliola torulosiseta

Hansf. 1957

Meliola bignoniacearum

F. Stevens 1928

Meliola tecomae $\mathrm{F}$.

Stevens 1916

Meliola bidentata Cooke

1882

Meliola ophidiochaeta

Cif. 1954

Asteridiella tabebuiae Hansford

(Bat. \& J. Silva) Hansf.

1961
Hansford

$1957 b$

Stevens 1928

Stevens 1928

Stevens 1928

Cooke 1882

Hansford

$1956 b$

Stevens 1928

Stevens 1928

Hansford

1955

Hansford

1955

Hansford

1961

Ciferri 1933

Stevens \&

Tehon 1926

Hansford

1955

Stevens 1928

Hansford

$1956 \mathrm{~b}$

Hansford

1937

Hansford

1937

Sydow \&

Sydow 1914d

Hansford \&

Deighton

1948

Hansford

1961

Hansford

$1956 \mathrm{~b}$

Stevens 1928

Stevens 1916

Cooke 1882

Ciferri 1954

1961 


\begin{tabular}{|c|c|c|c|}
\hline \multirow[t]{2}{*}{ Bixaceae } & Bixa orellana & $\begin{array}{l}\text { Meliola bixae Hansf. } \\
1955\end{array}$ & $\begin{array}{l}\text { Hansford } \\
1953 b\end{array}$ \\
\hline & $\begin{array}{l}\text { Cochlospermum regium } \\
\text { (Cochlospermum insigne) }\end{array}$ & $\begin{array}{l}\text { Meliola cochlospermifolii } \\
\text { Bat. } 1960\end{array}$ & $\begin{array}{l}\text { Batista et al. } \\
1960\end{array}$ \\
\hline \multirow[t]{21}{*}{ Boraginaceae } & Cordia ecalyculata (Cordia & Asteridiella cordiae- & Hansford \\
\hline & salicifolia) & salicifoliae Hansf. 1957 & $1956 b$ \\
\hline & Cordia heterophylla & $\begin{array}{l}\text { Asteridiella usteriana } \\
\text { (Rehm) Hansf. } 1961\end{array}$ & $\begin{array}{l}\text { Hansford } \\
1961\end{array}$ \\
\hline & Cordia nitida & $\begin{array}{l}\text { Asteridiella longipoda } \\
\text { (Gaillard) Hansf. } 1961\end{array}$ & $\begin{array}{l}\text { Hansford } \\
1961\end{array}$ \\
\hline & Cordia obliqua\# & !Asteridiella cordiae & Hansford \\
\hline & & Hansf. \& Deighton 1957 & $1956 b$ \\
\hline & $\begin{array}{l}\text { Cordia polycephala (Varronia } \\
\text { corymbosa) }\end{array}$ & $\begin{array}{l}\text { Asteridiella longipoda } \\
\text { (Gaillard) Hansf. } 1961\end{array}$ & $\begin{array}{l}\text { Hansford } \\
1961\end{array}$ \\
\hline & Cordia rufescens & Meliola cordiae- & Hansford \\
\hline & & $\begin{array}{l}\text { rufescentis Hansf. \& Bat. } \\
1957\end{array}$ & $1956 b$ \\
\hline & Cordia sp. & $\begin{array}{l}\text { Meliola cordiicola Hansf. } \\
1957\end{array}$ & $\begin{array}{l}\text { Hansford } \\
1957 b\end{array}$ \\
\hline & Ehretia cymosa & $\begin{array}{l}\text { Asteridiella mathengeana } \\
\text { Mibey } 1996\end{array}$ & $\begin{array}{l}\text { Mibey et al. } \\
1996\end{array}$ \\
\hline & Ehretia laevis (Ehretia & Asteridiella ehretiae & Hosagoudar et \\
\hline & canarensis) & Hosag. \& Raghu 1994 & al. $1994 b$ \\
\hline & Ehretia laevis (Ehretia & Meliola ehretiicola & Hosagoudar \\
\hline & canarensis) & Hosag. 1996 & 1996 \\
\hline & $\begin{array}{l}\text { Ehretia resinosa (Ehretia } \\
\text { navesii) }\end{array}$ & Meliola ehretiae Hansf. & $\begin{array}{l}\text { Hansford } \\
1055\end{array}$ \\
\hline & Tournefortia ramosissima & Asteridiella longipoda & Hansford \\
\hline & & (Gaillard) Hansf. 1961 & $\begin{array}{l}1961 \\
\text { Hansford }\end{array}$ \\
\hline & Tournefortia sp. & $\begin{array}{l}\text { Asteridiella longipoda } \\
\text { (Gaillard) Hansf. } 1961\end{array}$ & $\begin{array}{l}\text { Hansford } \\
1961\end{array}$ \\
\hline & Varronia sp. & $\begin{array}{l}\text { Irenopsis moelleriana (G. } \\
\text { Winter) F. Stevens } 1927\end{array}$ & Stevens 1927 \\
\hline & Varronia sp. & $\begin{array}{l}\text { Irenopsis varroniae } \\
\text { (Deighton) Hansf. } 1961\end{array}$ & $\begin{array}{l}\text { Hansford } \\
1961\end{array}$ \\
\hline \multirow[t]{2}{*}{ Brassicaceae } & Lunaria sp. & $\begin{array}{l}\text { Meliola bonaoensis Cif. } \\
1954\end{array}$ & Ciferri 1954 \\
\hline & Lunasia amara* & $\begin{array}{l}\text { Meliola patens Syd. \& P. } \\
\text { Syd. } 1912\end{array}$ & $\begin{array}{l}\text { Sydow \& } \\
\text { Sydow } 1912 b\end{array}$ \\
\hline \multirow[t]{12}{*}{ Burseraceae } & - & $\begin{array}{l}\text { Meliola torulosispora } \\
\text { M.L. Farr } 1987\end{array}$ & Farr 1987 \\
\hline & Aucoumea klaineana & Meliola aисоитеае J.M. & Index \\
\hline & & Yen \& Sulmont 1973 & $\begin{array}{l}\text { Fungorum } \\
2016\end{array}$ \\
\hline & Canarium album & $\begin{array}{l}\text { Meliola canarii-albi B. } \\
\text { Song \& Y.X. Hu } 1997\end{array}$ & $\begin{array}{l}\text { Song et al. } \\
1997 \mathrm{~b}\end{array}$ \\
\hline & Canarium asperum & Meliola canarii Syd. \& P. & Sydow \& \\
\hline & (Canarium villosum) & $\begin{array}{l}\text { Syd. } 1914 \\
\text { Meliola canarifolia }\end{array}$ & $\begin{array}{l}\text { Sydow 1914a } \\
\text { Hosaooudar et }\end{array}$ \\
\hline & cana & Hosag. et al. 2014 & $\begin{array}{l}\text { Hosagouaret } \\
\text { al. } 2014\end{array}$ \\
\hline & Canarium sp. & $\begin{array}{l}\text { Meliola nigrorufescens } \\
\text { Sacc } 1917\end{array}$ & $\begin{array}{l}\text { Saccardo } \\
1917\end{array}$ \\
\hline & Canarium sp. & Setameliola canariicola & Reynolds \\
\hline & & $\begin{array}{l}\text { (F. Stevens ex Hansf.) } \\
\text { D.R. Reynolds } 2010\end{array}$ & 2010 \\
\hline & Garuga floribunda (Garuga & Meliola garugae $\mathrm{F}$. & Hansford \\
\hline & & Hansf. 1963 & \\
\hline
\end{tabular}


Garuga sp.

Protium fragrans

Protium heptaphyllum

Protium panamense

Protium warmingianum*

Tetragastris balsamifera*

Tetragastris panamensis

Buxaceae

Cactaceae

Calophyllaceae

Campanulaceae

Canellaceae

Cannabaceae
Buxus glomerata

Buxus microphylla ( var. japonica)

Buxus sp.

Buxus sp.

Opuntia tuna

Mammea africana

Mammea sp.

Mesua ferrea

Clermontia persicifolia

Clermontia sp.

Cyanea argentea* ${ }^{*}$ Rollandia argentea)

Lobelia sp.

Lobelia stricta

Canella sp.

Cinnamodendron axillare

Warburgia ugandensis

Celtis iguanaea (Celtis aculeata)
Meliola garugae $\mathrm{F}$.

Hansford

Stevens \& Roldan ex

1962

Hansf. 1963

Meliola protiicola Mig.

Rodr. 2000

Asteridiella protiicola

(Bat. \& Gayão) Hansf.

1961

Meliola protii F. Stevens Stevens 1928

1928

!Meliola garugae var. Pinho et al. protii D.B. Pinho \& O.L. 2012

Pereira 2012

Meliola burseracearum F. Stevens 1928

Stevens 1928

Meliola burseracearum F. Stevens 1928

Stevens 1928

Meliola buxi Cif. $1938 \quad$ Ciferri 1938

Appendiculella buxi (I. Katumoto

Hino \& Katum.) Katum. 2010

2010

Meliola buxicola Doidge Doidge \&

1928

Sydow 1928

Meliola wenshanensis Jiang 1986b

G.Z. Jiang 1987

Meliola opuntiae Hansf. Hansford

1957

$1956 b$

Meliola mammeicola Hansford

Hansf. 1945 1945b

Meliola mammeae Hansf. Hansford

1946

1946

Meliola mesuae Hosag. et Hosagoudar et al. 2010

al. $2010 \mathrm{c}$

Appendiculella

Höhnel 1919

larviformis (Henn.) Höhn.

1919

Amazonia clermontiae

Hansf. 1957

Meliola lobeliae F.

Stevens 1974

Irenopsis rollandiae

Hansf. 1957

Meliola

campanulacearum Mig.

Rodr. \& Camino 1987

Meliola lobeliicola M.L. Farr 1969

Hansford

$1957 b$

Index

Fungorum

2016

Hansford

1957b

Rodríguez

Hernández \&

Camino

Vilaró 1987

Index

Fungorum

2016

Meliola canellae Cif.

Ciferri 1933

1933

Meliola cinnamodendri Stevenson

J.A. Stev. 1943

Meliola warburgiae

Mibey 1999

1943

Mibey \&

Kokwaro

1999

Meliola cabellensis Syd. $\quad$ Sydow 1930 
Celtis iguanaea (Celtis

glycycarpa)

Celtis luxensis

Celtis prantlii

Celtis timorensis (Celtis

cinnamomea)

Celtis zenkeri (Celtis soyauxii)

Celtis zenkeri (Celtis soyauxii)

Lozanella enantiophylla

Cannaceae

Capparaceae e
Caprifoliaceae

\section{Boscia caffra*}

Capparis acutifolia subsp.

Sabiifolia (Capparis kikuchii)

Capparis divaricata

Capparis erythrocarpos

Capparis rotundifolia

(Capparis pedunculosa)

Cynophalla flexuosa

(Capparis flexuosa)

Forchhammeria pallida

Maerua duchesnei (Capparis afzelii)

Quadrella cynophallophora

(Capparis cynophallophora)

Quadrella cynophallophora

(Capparis cynophallophora)

Stixis suaveolens

Ebulum formosana

Leycesteria glaucophylla

Lonicera glabrata*

Lonicera japonica

Lonicera japonica

Cardiopteridacea Citronella moorei

Citronella paniculata

(Villaresia megaphylla)
Meliola celtidum Speg. 1924

Meliola celtidis H.S.

Yates 1918

Meliola celtidis H.S.

Yates 1918

Meliola celtidicola H.S. Yates 1918

Yates 1918

!Meliola celtidicola $\mathrm{V}$ an der Byl 1926

Yates 1918

Appendiculella lozanellae

Rodr. Just. \& M.

Piepenbr. 2007

Meliola velutina $\mathrm{G}$.

Winter 1886

Asteridiella capparigena

Hosag. 2006

Irenopsis bosciae

(Doidge) F. Stevens 1927

Meliola sawadae B. Song

2004

Meliola capparicola

Hosag. 1996

Asteridiella capparis

(Hansf. \& Deighton)

Hansf. 1961

Meliola balakrishnanii

L.N. Nair \& V.P. Kaul

1983

Irenopsis ciferriana

(Petr.) Hansf. 1961

Asteridiella

forchhammeriae (Hansf.)

Hansf. 1961

Meliola capparis Hansf.

1946

Irenopsis capparicola

Bat. \& A.F. Vital 1955

Irenopsis martynii Hansf.

1957

Meliola stixis B. Song \&

T.H. Li 2002

Asteridiella ebuli (W.

Yamam.) Hansf. 1961

Meliola leycesteriae A.K.

Kar \& Maity 1970

Irenopsis glabratae Jana et al. 2005

Appendiculella lonicerae

B. Song 2002

Asteridiella lonicerae (W.

Yamam.) Hansf. 1961

Meliola citronellae Hansf.

1953

Meliola villaresiicola

Speg. 1924
Byl 1926

Yates 1918

Spegazzini

1924

Yates 1918

Rodríguez

Justavino \&

Piepenbring

2007

Index

Fungorum

2016

Hosagoudar et al. 2006c

Stevens 1927

Song \& $\mathrm{Li}$

2004a

Hosagoudar

1996

Hansford

1961

Nair \& Kaul

1983

Hansford

1961

Hansford

1961

Hansford

1946

Batista \&

Vital 1955

Hansford

1956b

Song et al.

2002d

Hansford

1961

Kar \& Maity

1970b

Jana et al.

2005d

Song et al.

2002c

Hansford

1961

Hansford

1953a

Spegazzini

1924
Yates 1918 
Villaresia sp.

Villaresia sp.

Villaresia sp.

Caricaceae

Carica papaya

Carica sp.

Carica sp.

Celastraceae

Cassine eucleiformis

(Mystroxylon euclaeforme)

Cassine eucleiformis

(Mystroxylon euclaeforme)

Celastrus cordatus*

Celastrus sp.

Celastrus sp.

Crossopetalum rhacoma

(Crossopetalum pallens)

Euonymus indicus

Euonymus sp.

Gymnosporia emarginata

(Maytenus emarginata)

Gymnosporia senegalensis

Gymnosporia sp.

Gymnosporia spinosa*

Hippocratea ?richardiana

Hippocratea schlechteri*

Hippocratea sp.

Hippocratea volubilis

Loeseneriella clematoides

(Hippocratea clematoides)

Lophopetalum sp.

Lophopetalum toxicum*
Meliola adunciseta Hansf. Hansford 1963

1962

Meliola villaresiae Henn. Hennings

1897

1897

Meliola villaresiana

Hansf. 1957

Meliola papayae

Deighton 1968

Hansford

$1957 b$

Deighton

1968

Irenopsis moelleriana (G. Stevens 1927

Winter) F. Stevens 1927

Meliola caricis Hansf.

1958

Hansford

$1957 \mathrm{c}$

Irenopsis laevis (Berk. \& Hansford

M.A. Curtis) Hansf. 19551955

Meliola celastrigena

Hosag. 2013

Hosagoudar

$2013 b$

Meliola neelikalluensis Hosagoudar

Hosag. \& B. Divya 2013 \& Divya 2013

Meliola evansii Doidge Doidge 1920b

1920

Meliola pterocelastri J.L. Crane \&

Crane \& A.G. Jones $2001 \quad$ Jones 2001

Appendiculella natalensis Hansford

(Doidge) Hansf. 1961

\#Asterina ditricha

Kalchbr \&

Kalchbr. \& Cooke 1880

Amazonia celastri Y.X.

Hu \& B. Song 1997

Irenopsis compacta

(Earle) F. Stevens 1927

Meliola euonymicola

Hosag. 1996

Cooke 1880

Song et al.

1997e

Stevens 1927

Meliola euonymi $\mathrm{F}$.

Hosagoudar

1996

Hansford

Stevens ex Hansf. 1957 1957b

Amazonia patilii Hosag. Hosagoudar

1996

1996

Irenopsis gymnosporiae Hansford

Hansf. 1938

1938

Appendiculella speciosa

(Doidge) Hansf. 1961

Asteridiella gymnosporiae

(Syd. \& P. Syd.) Hansf.

1961

Meliola hippocrateicola

Hansf. \& Deighton 1948

Meliola hippocrateae

Doidge 1948

Meliola reinkingii Syd. \&

P. Syd. 1920

Meliola torulipes Cif.

1938

Asteridiella hippocrateae Hansford

(Hansf. \& Deighton) 1961

Hansf. 1961

Meliola chenniana Hosag. Goos \& \& Goos 1998

Hosagoudar

1998

Meliola lophopetali

Hansford

Hansf. 1957 
Lophopetalum wightianum*

Lophopetalum wightianum*

Maytenus rothiana*

Microtropis sp.

Moya ferox*

Pleurostylia sp.

Pleurostylia sp.

Rhacoma crossopetalum

Rhacoma crossopetalum

Rhacoma sp.

Salacia arborea (Salacia undulata)

Salacia elegans

Salacia erecta

Salacia sp.

Schaefferia frutescens

Centroplacaceae Bhesa indica

Chloranthaceae Chloranthus elatior (Chloranthus officinalis)

Chrysobalanacea e

Chrysobalanus sp.

Hirtella zanzibarica

Clethraceae

Clusiaceae
Asteridiella lophopetali Hosag. \& Raghu 1993

Meliola lophopetaligena Hosag. \& P.J. Robin 2011

Amazonia mayteni Hosag. et al. 1994

Meliola microtropidis

Hosag. \& B. Fathima 2013

Meliola falcatiseta Speg. 1909

Meliola celastracearum

Hosag. \& B.R. Dayal

1996

Meliola pleurostyliicola

Hosag. \& T. Sabu 2007

Irenopsis tehoniana

(Trotter) Hansf. 1961

Meliola earleana Cif.

1954

Irenopsis conferta

(Tehon) F. Stevens 1927

!Meliola montagnei Pat.

Meliola salaciae Hansf. 1946

Asteridiella salaciae-

erectae Deighton 1958

Meliola salaciicola

Hosag. et al. 2007

Asteridiella toroana (Cif.)

Hansf. 1961

Meliola bhesae Hosag.

1991

Asteridiella chloranthi $\mathrm{F}$.

Stevens ex Hansf. 1957

Meliola

chrysobalanacearum

M.L. Farr 1987

Meliola spinksii Dilcher 1965

Meliola hirtellae Mibey 1997

Asteridiella clethrae

Hansf. 1963

Asteridiella chardonii

(Toro) S. Hughes 1993

Meliola cyrillacearum

Schmied. 1989

Asteridiella galipanensis

Toro ex Hansf. 1957

Meliola brevispora M.L.

Farr 1987
Hosagoudar

\& Raghu

1993

Hosagoudar

\& Robin

2011b

Hosagoudar et

al. 1994 a

Hosagoudar

\& Fathima

2013

Spegazzini

1909

Hosagoudar

1996

Hosagoudar et

al. $2007 \mathrm{~b}$

Hansford

1961

Ciferri 1954

Stevens 1927

Index

Fungorum

2016

Hansford

1946

Deighton

1957

Hosagoudar et al. $2007 \mathrm{a}$

Hansford

1961

Hosagoudar

\& Goos 1991

Hansford

1957b

Farr 1987

Index

Fungorum

2016

Mibey \&

Hawksworth

1997

Hansford

1962

Hughes 1993

Schmiedeknec

ht 1989

Hansford

1956b

Farr 1987 
Calophyllum apetalum

Calophyllum brasiliense var. antillanum (Calophyllum antillanum)

Calophyllum calaba

Calophyllum sp.

Clusia minor

Clusia sp.

Garcinia kydia

Garcinia morella

Garcinia oligantha

Garcinia sp.

Ochrocarpos longifolius

Symphonia globulifera

Symphonia globulifera (Symphonia gabonensis)

Combretaceae Buchenavia tetraphylla

(Buchenavia capitata)

Combretum decandrum

Combretum farinosum

Combretum indicum

(Quisqualis indica)

Combretum latifolium

(Combretum extensum)

Combretum molle

(Combretum velutinum)

Combretum mooreanum

Combretum mucronatum

Combretum platypterum

Combretum racemosum

Combretum sp.

Getonia floribunda (Calycopteris floribunda)

Laguncularia racemosa

Laguncularia racemosa

Lumnitzera racemosa
Appendiculella calophylli (F. Stevens) Toro 1925

Appendiculella calophylli Toro 1925 (F. Stevens) Toro 1925

Appendiculella calophylli (F. Stevens) Toro 1925 Appendiculella calophylli (F. Stevens) Toro 1925

Meliola clusiae F. Stevens Stevens 1916 1916

Amazonia clusiae F.

Stevens 1927

Meliola kydia Sacc. 1921 Saccardo

1921

Meliola garcinigena

Hosag. \& A. Sabeena

2013

Hosagoudar

\& Sabeena

$2013 a$

Meliola garciniicola G.Z. Jiang 1986a

Jiang 1987

Meliola garciniae H.S. Yates 1918

Yates 1918

Meliola ochrocarpi Thite Thite \& Patil

\& S.D. Patil $1983 \quad 1983$

!Meliola symphoniae Bat. Batista et al.

\& H. Maia $1965 \quad$ 1965a

Asteridiella symphoniae Hansford

(Hansf.) Hansf. 1961

Meliola buchenaviae Bat. Index 1953

Fungorum

2016

Amazonia henryi Hosag. Hosagoudar

1989

$1988 \mathrm{a}$

Asteridiella combreti (F. Hansford

Stevens) Hansf. $1961 \quad 1961$

Asteridiella combreti (F. Hansford

Stevens) Hansf. $1961 \quad 1961$

Asteridiella combreti (F. Hansford

Stevens) Hansf. $1961 \quad 1961$

Asteridiella combreti (F. Hansford

Stevens) Hansf. $1961 \quad 1961$

Asteridiella combreti (F. Hansford

Stevens) Hansf. $1961 \quad 1961$

Asteridiella combreti (F. Hansford

Stevens) Hansf. $1961 \quad 1961$

Asteridiella combreti (F. Hansford

Stevens) Hansf. $1961 \quad 1961$

Asteridiella combreti (F. Hansford

Stevens) Hansf. $1961 \quad 1961$

Meliola sudanensisHansf. Hansford 1957

Meliola calycopteridis

Hosag. et al. 2013

Asteridiella lagunculariae

(Earle) Hansf. 1961

Asteridiella nigra (F.

Stevens) Hansf. 1961

Meliola pelliculosa Syd.

\& P. Syd. 1913
$1956 \mathrm{~b}$

Hosagoudar 2013a

Hansford

1961

Hansford

1961

Sydow \&

Sydow 1914b 
Terminalia ivorensis

Terminalia superba

Terminalia tomentosa

Connaraceae

Agelaea pentagyna (Agelaea ugandensis)

Agelaea pentagyna (Agelaea ugandensis)

Agelaea pentagyna (Agelaea ugandensis)

Agelaea sp.

Cnestis polyphylla (Cnestis natalensis)

Connarus africanus

Connarus panamensis

Connarus sclerocarpus

Connarus sp.

Ellipanthus

hemandradenioides

Manotes expansa (Manotes longiflora)

Rourea erecta*

Rourea minor (Santaloides afzelii)

Rourea prainiana*

Rourea prainiana*

Rourea surinamensis

?Convolvulaceae

Convolvulaceae

Argyreia hookeri*

Bonamia cymosa

Breweria sp.

Convolvulus nodiflorus

(Jacquemontia nodiflora)

Cuscuta sp.
Asteridiella terminaliae (Hansf. \& Deighton)

Hansf. 1961

Asteridiella combreti (F. Hansford Stevens) Hansf. 1961

1961

Meliola buchenaviae Bat. Index 1953

Fungorum

2016

\#Meliola agelaeae Hansf. Hansford 1938

1938

Meliola agelaeicola

Hansf. 1946

Meliola mabirensis

Hansf. 1946

!Meliola agelaeae $\mathrm{F}$.

Stevens \& Roldan 1935

Meliola cnestidis Doidge

1928

Meliola roureae Syd. \&

P. Syd. 1917

Meliola connari H.S.

Yates 1917

Meliola connari H.S.

Yates 1917

Meliola connari H.S.

Yates 1917

Meliola ellipanthi Mibey 1997

Meliola manotis Hansf. \& Deighton 1948

Hansford

1946

Hansford

1946

Stevens \&

Roldan 1935

Doidge \&

Sydow 1928

Sydow \&

Sydow 1917a

Yates 1917

Yates 1917

Yates 1917

Mibey \&

Hawksworth

1997

Hansford \&

Deighton

1948

Meliola roureae Syd. \&

P. Syd. 1917

Sydow \&

Sydow 1917a

Meliola roureae Syd. \& Sydow \&

P. Syd. 1917

Sydow 1917a

!Irenopsis agumbensis A. Pande 2008

Pande 2008

Irenopsis agumbensis

Subhedar \& V.G. Rao

1977

Meliola roureae Syd. \&

P. Syd. 1917

Meliola decidua Speg.

1889

Subhedar \&

Rao 1977

Sydow \&

Sydow 1917a

Spegazzini

$1888 \mathrm{a}$

Meliola densa Cooke 1884

Meliola argyreiae M.S. Patil \& Thite

Patil \& Thite 1975

1974

Meliola bonamiae Hansf. Hansford \& \& Deighton 1948

Meliola francevilleana

Gaillard 1892

Meliola francevilleana

Gaillard 1892

Meliola cuscutae Hansf. 1958
Deighton

1948

Index

Fungorum

2016

Index

Fungorum

2016

Hansford

$1957 \mathrm{c}$ 
Dichondra repens

Erycibe henryi

Erycibe paniculata*

Erycibe rhedii

Evolvulus sp.

Hewittia malabarica

(Hewittia sublobata)

Ipomoea batatas

Ipomoea crassicaulis*

Ipomoea pes-caprae

Ipomoea pes-caprae

Ipomoea prope\#

Ipomoea sp.

Ipomoea sp.

Ipomoea sp.

Ipomoea sp.

Ipomoea $\mathrm{sp}$.

Ipomoea! sp.

Lepistemon owariensis*

Merremia tridentata

(Merremia hastata)

Cornaceae

Costaceae

Cucurbitaceae
Alangium chinense (Alangium begoniifolium)

Alangium salviifolium

Mastixia arborea

Mauritiella armata (Mauritia martiana)

Costus afer

Costus sp.

Costus sp.

Costus spicatus (Costus

cylindricus)

Monocostus uniflorus (Dimerocostus uniflorus)
Meliola malacotricha

Speg. 1888

Meliola erycibicola

Sawada \& W. Yamam.

1959

Meliola erycibes-

paniculatae Hosag. 1990

Meliola erycibes Hansf.

1954

Meliola ambigua Pat. \&

Gaillard 1888

!Meliola hewittiae Rehm

1913

Meliola clavulata $\mathrm{G}$. Winter 1886

Winter 1886

Meliola malacotricha

Speg. 1888

Spegazzini

$1888 b$

!Meliola ipomoeae Bat. Batista 1946

1946

Meliola pontualii A.F. Viégas 1947

Vital 1947

Meliola ipomoeae Earle Earle 1901

1901

Meliola ipomoeicola

Beeli 1920

Meliola pallida F. Stevens Stevens 1928 1928

Meliola permixta Syd. \& Sydow 1923b

P. Syd. 1923

Meliola quadrifurcata Rehm 1913c

Rehm 1913

Meliola quadrispina

Racib. 1900

Index

Fungorum

2016

Irenopsis comata

Stevens 1927

(Doidge) F. Stevens 1927

Meliola lepistemonis

Hansford

Hansf. 1937

1937

Meliola merremiae Rehm Rehm 1913a

1913

Meliola alangii Syd. \& P. Sydow \&

Syd. 1916

Meliola alangii Syd. \& P.

Syd. 1916

Asteridiella mastixiae

Hosag. \& Raghu 1996

Asteridiella manaosensis

(Henn.) Hansf. 1961

Irenopsis chandleri

Hansf. 1941

Sydow 1916b

Sydow \&

Sydow $1916 b$

Hosagoudar

1996

Hansford

1961

Hansford

1941

Asteridiella costi $(\mathrm{F}$.

Stevens) Hansf. 1961

Hansford

1961

Asteridiella parasitica (F. Hansford

Stevens) Hansf. $1961 \quad 1961$

Meliola longistipitata F. Stevens 1928

Stevens 1928

Meliola longistipitata F. Stevens 1928

Stevens 1928

Irenopsis aciculosa (G.

Winter) F. Stevens 1927
Stevens 1927 
Anguria sp.*

?Cayaponia sp.

Coccinia barteri (Physedra barteri)

Cogniauxia podolaena

(Cogniauxia cordifolia)

Cucumeropsis mannii

(Cucumeropsis edulis)

Cucurbita sp.

Cyclanthera leptostachya

Luffa cylindrica

Melothria trilobata

Trichosanthes quinquangulata

Zehneria obtusiloba*

Cunoniaceae Ceratopetalum apetalum

Ceratopetalum apetalum

Codia spatulata

Cunonia capensis

Cupania americana

Cupania vernalis

Cupania vernalis

Mischocarpus sundaicus

(Cupania revoluta)

Pancheria sp.*

Cupressaceae Austrocedrus chilensis

Cunninghamia lanceolata

Fitzroya cupressoides

Pilgerodendron uviferum
Meliola malacotricha

Speg. 1888

Spegazzini

$1888 b$

Asteridiella anguriae (F. Hansford

Stevens) Hansf. $1961 \quad 1961$

Meliola cucurbitacearum Stevens 1916

F. Stevens 1916

Asteridiella triloba (G. Hansford

Winter) Hansf. $1961 \quad 1961$

Meliola cogniauxiae Hansford

Hansf. 1957 1957b

Asteridiella triloba (G. Hansford

Winter) Hansf. $1961 \quad 1961$

Asteridiella confragosa Hansford

(Syd. \& P. Syd.) Hansf. 1961

1961

Meliola cyclantherae Syd. Sydow 1930

1930

Asteridiella confragosa Hansford

(Syd. \& P. Syd.) Hansf. 1961

1961

Irenopsis melothriae

R.E.D. Baker \& W.T.

Dale 1951

Asteridiella confragosa

(Syd. \& P. Syd.) Hansf.

1961

Irenopsis zehneriae (Van

der Byl) F. Stevens 1927

Appendiculella megalongensis (Hansf.)

Hansf. 1961

Meliola ceratopetali Hansford

Hansf. 1953 1953a

Setameliola codiae (B. Reynolds

Huguenin) D.R. Reynolds 2010

2010

Meliola cunoniae Hansf. Hansford

1955

1955

Meliola capensis

Theissen 1912

(Kalchbr. \& Cooke)

Theiss. 1912

Meliola capensis

(Kalchbr. \& Cooke)

Theiss. 1912

Meliola vernalis Pinho et al. 2012

Meliola cupaniicola Bat. 1960

Baker \& Dale 1951

Hansford

1961

Stevens 1927

Hansford

1961

Amazonia pancheriae $\mathrm{B}$.

Huguenin 1969

Appendiculella

austrocedri Butin 1974

Appendiculella

cunninghamiae Y.X. Hu

\& B. Song 1992

Appendiculella fitzroyae

Butin 1974

Appendiculella

pilgerodendri Butin 1974
Theissen 1912

Pinho et al.

2012

Batista et al.

1960

Huguenin

1969

Butin 1974

Hu \& Song

1992

Butin 1974
Butin 1974 


\begin{tabular}{|c|c|c|c|}
\hline Curtisiaceae & Curtisia faginea & $\begin{array}{l}\text { Meliola ganglifera } \\
\text { Kalchbr. } 1880\end{array}$ & $\begin{array}{l}\text { Kalchbrenner } \\
\text { \& Cooke } \\
1880\end{array}$ \\
\hline \multirow[t]{3}{*}{ Cyclanthaceae } & Asplundia rigida & Meliola carludovicae & Hansford \\
\hline & (Carludovica plumeri) & Hansf. 1955 & 1955 \\
\hline & Carludovica sp. & Meliola carludovicae & Hansford \\
\hline \multirow[t]{23}{*}{ Cyperaceae } & Actinoscirpus grossus & Meliola intricata Syd. \& & Sydow \& \\
\hline & (Scirpus grossus) & P. Syd. 1913 & Sydow 1913a \\
\hline & Chorizandra cymbaria & Meliola chorizandrae B. & Index \\
\hline & & Huguenin 1969 & $\begin{array}{l}\text { Fungorum } \\
2016\end{array}$ \\
\hline & Cladium mariscus & $\begin{array}{l}\text { Meliola italica (Sacc.) F. } \\
\text { Stevens } 1928\end{array}$ & Stevens 1928 \\
\hline & $\begin{array}{l}\text { Cladium mariscus subsp. } \\
\text { jamaicense (Cladium } \\
\text { occidentale) }\end{array}$ & $\begin{array}{l}\text { Setameliola apiculata } \\
\text { (Hansf.) D.R. Reynolds } \\
2010\end{array}$ & $\begin{array}{l}\text { Reynolds } \\
2010\end{array}$ \\
\hline & Cyperus flabelliformis\# & $\begin{array}{l}\text { Setameliola argentina } \\
\text { (Speg.) D.R. Reynolds } \\
2010\end{array}$ & $\begin{array}{l}\text { Reynolds } \\
2010\end{array}$ \\
\hline & Cyperus sp. & $\begin{array}{l}\text { Meliola cyperacearum } \\
\text { Hosag. et al. } 2011\end{array}$ & $\begin{array}{l}\text { Nithyatharani } \\
\text { et al. } 2011\end{array}$ \\
\hline & Cyperus sp. & Setameliola cyperi (Pat.) & Reynolds \\
\hline & Eleocharis sp. & $\begin{array}{l}\text { D.R. Reynolds } 2010 \\
\text { Meliola uleana Pazschke }\end{array}$ & $\begin{array}{l}2010 \\
\text { Index }\end{array}$ \\
\hline & & 1892 & $\begin{array}{l}\text { Fungorum } \\
2016\end{array}$ \\
\hline & $\begin{array}{l}\text { Gahnia beecheyi (Gahnia } \\
\text { leptostachya) }\end{array}$ & $\begin{array}{l}\text { Setameliola argentina } \\
\text { (Speg.) D.R. Reynolds } \\
2010\end{array}$ & $\begin{array}{l}\text { Reynolds } \\
2010\end{array}$ \\
\hline & Mapania sp. & $\begin{array}{l}\text { Meliola mapaniae H.S. } \\
\text { Yates } 1917\end{array}$ & Yates 1917 \\
\hline & Plumaria sp. * & $\begin{array}{l}\text { \#Meliola plumeriae } \\
\text { Srinivas. } 1974\end{array}$ & $\begin{array}{l}\text { Srinivasulu } \\
1974\end{array}$ \\
\hline & Remirea maritima & $\begin{array}{l}\text { Meliola remireae Bat. } \\
1960\end{array}$ & $\begin{array}{l}\text { Batista et al. } \\
1960\end{array}$ \\
\hline & Rhynchospora corymbosa & $\begin{array}{l}\text { Meliola tibigirica Hosag. } \\
\text { \& T.K. Abraham } 1997\end{array}$ & $\begin{array}{l}\text { Hosagoudar et } \\
\text { al. } 1997\end{array}$ \\
\hline & Rhynchospora corymbosa & Setameliola circinans & Reynolds \\
\hline & (Rhynchospora aurea) & $\begin{array}{l}\text { (Earle) D.R. Reynolds } \\
2010\end{array}$ & 2010 \\
\hline & Rhynchospora megalocarpa & Setameliola circinans & Reynolds \\
\hline & (Rhynchospora dodecandra) & $\begin{array}{l}\text { (Earle) D.R. Reynolds } \\
2010\end{array}$ & 2010 \\
\hline & Rhynchospora miliacea & Meliola caricis Hansf. & Hansford \\
\hline & Scleria so. & $\begin{array}{l}1958 \\
\text { Setameliola aniculata }\end{array}$ & $\begin{array}{l}1957 \mathrm{c} \\
\text { Revnolds }\end{array}$ \\
\hline & & $\begin{array}{l}\text { (Hansf.) D.R. Reynolds } \\
2010\end{array}$ & 2010 \\
\hline \multirow[t]{3}{*}{$\begin{array}{l}\text { Daphniphyllacea } \\
\text { e }\end{array}$} & Daphniphyllum calycinum & $\begin{array}{l}\text { Asteridiella } \\
\text { daphniphyllicola B. Song } \\
\text { et al. } 2000\end{array}$ & $\begin{array}{l}\text { Song et al. } \\
2000\end{array}$ \\
\hline & Daphniphyllum neilgherrense & $\begin{array}{l}\text { Amazonia daphniphylli } \\
\text { M.S. Patil } 1989\end{array}$ & $\begin{array}{l}\text { Hosagoudar et } \\
\text { al. } 1989\end{array}$ \\
\hline & Daphniphyllum paxianum & $\begin{array}{l}\text { Meliola daphniphyllicola } \\
\text { B. Song \& T.H. Li } 2002\end{array}$ & $\begin{array}{l}\text { Song et al. } \\
2002 \mathrm{~d}\end{array}$ \\
\hline \multirow[t]{2}{*}{ Dichapetalaceae } & Dichapetalum gelonioides & Meliola dichapetali & Hansford \& \\
\hline & & Hansf. \& Thirum. 1948 & $\begin{array}{l}\text { Thirumalacha } \\
\text { r } 1948\end{array}$ \\
\hline
\end{tabular}


Dichapetalum toxicarium

Tapura sp.

Dicksoniaceae Dicksonia sp.

Dilleniaceae

Acrotrema sp.

Dillenia pentagyna

Dillenia suffruticosa (Wormia suffruticosa)

Doliocarpus sp.

Doliocarpus sp.

Doliocarpus sp.

Tetracera potatoria

Tetracera potatoria

Tetracera scandens

Tetracera wuthiana*

Wormia alata*

Dioscoreaceae Dioscorea scortechinii (var. parviflora)

Dioscorea sp.

Dioscorea sp.

Dioscorea sp.

Dioscorea sp.

Dioscorea $\mathrm{sp}$.

Dioscorea sp.

Dipentodontacea Perrottetia sandwicensis e

Perrottetia sandwicensis
Meliola scott-elliotii

Hansf. \& Deighton $1957 \quad 1956 b$

Asteridiella tapurae Mig. Rodríguez

Rodr. \& Camino 1987

Hernández \&

Camino

Vilaró 1987

Irenopsis cornuta (Rehm) Hansford

Hansf. 1955

1955

Asteridiella obscura (F. Hansford

Stevens) Hansf. $1961 \quad 1961$

Asteridiella thwaitesii Hansford

(Berk. ex Hansf.) Hansf. 1961

1961

Asteridiella dilleniae

Hosag. \& Kamar. 2001

Hosagoudar et al. 2001a

Meliola malacensis Sacc. Saccardo 1921

Appendiculella doliocarpi Hansford Hansf. 19551955

Asteridiella doliocarpi Hansford

(Hansf.) Hansf. 1961

Asteridiella

longipedicellata $(\mathrm{F}$.

Stevens) Hansf. 1961

Asteridiella tetracerae Hansford

(Hansf. \& Deighton) 1962

Hansf. 1963

Asteridiella tetracericola Hansford

Hansf. \& Deighton 1961

Asteridiella tetracerae

(Hansf. \& Deighton)

Hansf. 1963

Meliola tetracerae F.

Muell. \& Thüm. 1878

Meliola wormiae Hansf. 1953

Hansford

1961

1961

Hansford

1962

Index

Fungorum

2016

Hansford

$1953 \mathrm{a}$

Meliola lianchangensis

G.Z. Jiang 1995

Amazonia dioscoreae Hansford

Hansf. \& F. Stevens 19371937

Meliola dioscoreacearum Hosagoudar

Hosag. \& Jac. Thomas \& Thomas

2009

2009

Meliola dioscoreae

Hansf. \& Deighton 1948

Hansford \&

Deighton

1948

Meliola dioscoreicola

Hansford \&

Hansf. \& Deighton 1948

Deighton

1948

Meliola dioscoreigena

Hosag. \& Jac. Thomas

Hosagoudar

\& Thomas

2009

Meliola zambalesica Petr. Petrak 1958

1959

Amazonia perrottetiae F. Stevens 1925

Stevens 1925

Amazonia stevensii Hansf. Hansford

1955
1955 
Perrottetia sandwicensis

Perrottetia sandwicensis

Perrottetia sandwicensis

Perrottetia sp.

Dipterocarpaceae Hopea sp.

Ebenaceae Diospyros australis

Diospyros buxifolia

Diospyros buxifolia

Diospyros candolleana

Diospyros crassiflora

Diospyros discolor

Diospyros ebenum

Diospyros heudelotii

Diospyros heudelotii

Diospyros heudelotii

Diospyros kabuyeana

Diospyros montana

Diospyros pentamera

Diospyros riedelii

Diospyros sp.

Diospyros vera (Maba buxifolia)

Euclea divinorum

Euclea natalensis subsp. obovata (Euclea fructuosa)

Euclea racemosa (Euclea latidens)

Elaeagnaceae
Appendiculella

kalalauensis Hansf. 1957 1957b

Appendiculella

Hansford perrottetiae (F. Stevens) 1955

Hansf. 1955

Asteridiella waimeana Hansford

Hansf. 1957

$1957 b$

Meliola dallasica Petr. Petrak 1954

1954

Meliola hopeae H.S.

Yates 1918

Meliola diospyricola

Hansf. 1953

Meliola diospyri-

buxifoliae Hosag. et al.

2011

Yates 1918

Meliola megalocarpa

Syd. \& P. Syd. 1923

Asteridiella eucleae

(Hansf.) Hansf. 1961

Meliola hirsuta Hansf. \&

Deighton 1948

Hansford

$1953 \mathrm{a}$

Hosagoudar

\& Sabeena

2011a

Sydow 1923a

Hansford

1961

Hansford \&

Deighton

1948

Meliola yatesiana Trotter Saccardo 1926

1926

Meliola ebeni Hosag. \&

Archana 2010

Hosagoudar \& Archana

2010

Asteridiella diospyricola (Hansf. \& Deighton)

Hansf. 1961

Meliola diospyri Syd. \& P. Syd. 1911

Hansford

1961

Meliola hirsuta Hansf. \& Deighton 1948

Sydow et al.

1911

Hansford \&

Deighton

1948

Meliola muhakae Mibey

\& P.F. Cannon 1999

Mibey \&

Cannon 1999

Meliola diospyri Syd. \&

P. Syd. 1911

Meliola diospyri-

pentamerae Hansf. 1953

Meliola harrietensis

Hosag. et al. 2014

Asteridiella kapoorii

Hosag. \& Raghu 1996

Meliola megalocarpa

Syd. \& P. Syd. 1923

Meliola eucleae Hansf.

1941

Meliola fructicosae

Mibey 1997

Sydow et al.

1911

Hansford

1953a

Hosagoudar et

al. 2014

Hosagoudar

1996

Sydow 1923a

Hansford

1941

Mibey \&

Hawksworth

1997

Asteridiella eucleae

(Hansf.) Hansf. 1961

Hansford

1961

Meliola elaeagni Hansf.

\& Thirum. 1948
Hansford \&

Thirumalacha r 1948 
Elaeocarpaceae Elaeocarpus monocera

Elaeocarpus munroii

Elaeocarpus sp.

Elaeocarpus tuberculatus

Elaeocarpus tuberculatus

Elaeocarpus tuberculatus

Sloanea jaginea\#

Sloanea sinensis

Sloanea sinensis

Sloanea sp.

Ericaceae

\section{Agapetes serpens (Pentapterygium serpens) Agapetes sp. \\ Agarista salicifolia (Andromeda salicifolia) Agarista salicifolia (Andromeda salicifolia) Agarista salicifolia (Andromeda salicifolia) Agauria sp.}

Andromeda sp.*

Craibiodendron $\mathrm{sp}$.

Craibiodendron stellatum

Cyathodes glauca

Gaultheria fragrantissima

Gaultheria indica\#

Gaultheria sp.

Gaylussacia brasiliensis

Gaylussacia brasiliensis

Gaylussacia sp.

?Gaylussacia sp.
Asteridiella

Hansford elaeocarpicola Hansf. 1961

1961

Amazonia elaeocarpi

Hosag. et al. 2007

Hosagoudar et

Meliola elaeocarpi H.S. Yates 1917

Yates 1917

Appendiculella elaeocarpi Hosagoudar Hosag. \& P.J. Robin 2011 \& Robin $2011 b$

Appendiculella elaeocarpicola Hosag. \& P.J. Robin 2011 Hosagoudar \& Robin $2011 b$ Asteridiella elaeocarpi- Hosagoudar tuberculati Hosag. 1991 1991a Asteridiella amoena Hansford (Syd.) Hansf. 1961

Asteridiella sloaneae B. $\quad$ Song \& Li Song 2004 $2004 b$

Irenopsis sloaneicola B. Song et al.

Song et al. 2001

Asteridiella amoena

(Syd.) Hansf. 1961

2001

Hansford

1961

Asteridiella pentapterygii Kar \& Maity

A.K. Kar \& Maity $1971 \quad 1970 a$

Amazonia karii Hosag. \&

N.P. Balakr. 1989

Hosagoudar et

Asteridiella agauriae

(Hansf.) Hansf. 1961

Asteridiella andromedae

(Pat.) Hansf. 1961

al. 1989

Hansford

1961

Hansford

1961

Meliola agauriae Hansf.

1947

Hansford

$1947 b$

Appendiculella

camerunensis Hansf.

1957

Asteridiella andromedae

(Pat.) Hansf. 1961

Hansford

$1956 b$

Hansford

1961

Asteridiella craibiodendri

B. Song 2003

Song \& Li

2003

Asteridiella gaylussaciae Hansford

(Hansf.) Hansf. 1961

Meliola cyathodis Hansf.

1946

Hansford

1946

Appendiculella

Biju et al. gaultheriae Hosag. et al. 2005

Asteridiella gaultheriae Hosag. et al. 2005

2005

Biju et al. 2005

Asteridiella exilis (Syd. \& P. Syd.) Hansf. 1961

Hansford

1961

Asteridiella gaylussaciae

(Hansf.) Hansf. 1961

Hansford

1961

Asteridiella henningsii

(Beeli) Hansf. 1961

Hansford

1961

Meliola callosperma

Speg. 1919

Spegazzini

1919b

Asteridiella puiggariana

Hansf. 1957
Hansford

$1956 b$ 


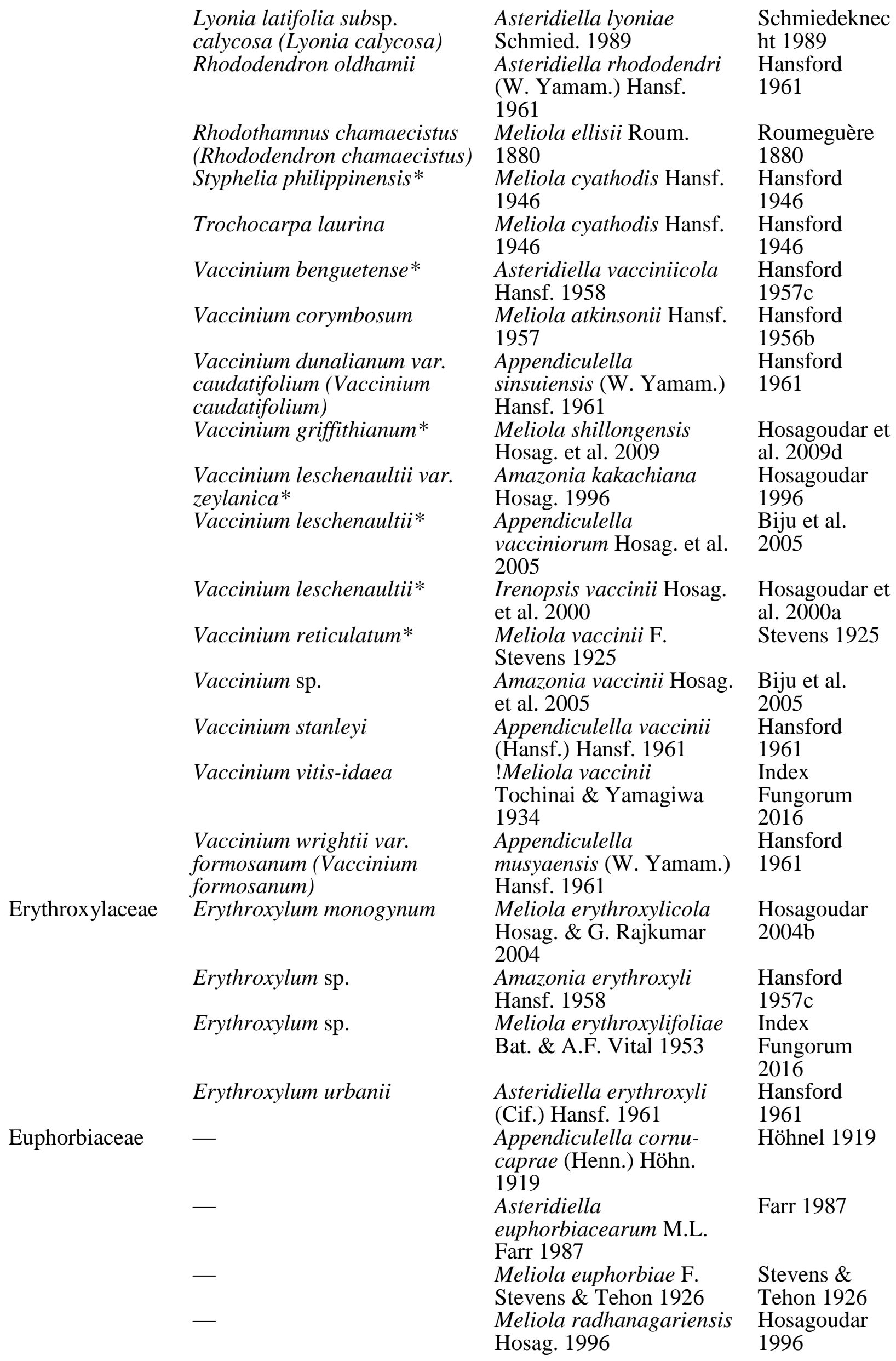


Acalypha?fruticosa

Acalypha bipartita

Acalypha bisetosa

Acalypha diversifolia

Acalypha sp.

Acalypha sp.

Acalypha sp.

Acalypha stipulacea\#

Adelia ricinella

Agrostistachys borneensis

Alchornea cordifolia

Alchornea cordifolia

Alchornea cordifolia (Alchornea cordata) Alchornea cordifolia (Alchornea cordata) Alchornea hirtella

Alchornea incurva\#

Alchornea laxiflora

Alchornea rugosa (Alchornea javanensis)

Alchornea scandens (Malaisia scandens)

Alchornea sp.

Argomuellera macrophylla

Balakata baccata (Sapium baccatum)

Claoxylon sandwicense

Codiaeum variegatum

Colliguaja brasillensis
Meliola chandleri Hansf. Hansford 1937

1937

Asteridiella hansfordii (F. Hansford

Stevens) Hansf. $1961 \quad 1961$

Appendiculella

Höhnel 1919

larviformis (Henn.) Höhn.

1919

Appendiculella

Höhnel 1919

larviformis (Henn.) Höhn.

1919

!Meliola acalyphae Jana Jana \& Ghosh

\& S.N. Ghosh 2007

Irenopsis paulensis

Hansf. 1955

Meliola acalyphicola

Deighton 1968

Amazonia acalyphae

(Rehm) Theiss. 1917

Meliola jatrophae $\mathrm{F}$.

Stevens 1916

Meliola agrostistachydis

Hosag. \& G. Rajkumar

2005

Asteridiella entebbeensis

(Hansf. \& F. Stevens)

Hansf. 1961

Asteridiella hansfordii (F.

Stevens) Hansf. 1961

\#Irene alchorneae $\mathrm{F}$.

Stevens \& Tehon 1926

Meliola pseudoradiata

Cif. 1954

Asteridiella hansfordii (F.

Stevens) Hansf. 1961

Asteridiella alchorneae-

incurvatae (Hansf.)

Hansf. 1961

Meliola kakamegensis

Mibey \& P.F. Cannon

1999

Meliola alchorneicola

Hansf. 1954

Appendiculella malaisiae

B. Song 2004

Appendiculella

alchorneae (F. Stevens \&

Tehon) Hansf. 1961

Meliola argomuellerae

Hansf. 1944

Meliola sapiicola Y.X.

Hu \& B. Song 1997

Meliola morbosa $\mathrm{F}$.

Stevens 1925

Asteridiella entebbeensis

(Hansf. \& F. Stevens)

Hansf. 1961

Meliola colliguajae Speg.

1912
2007

Hansford

$1953 b$

Deighton

1967

Theissen 1916

Stevens 1916

Hosagoudar

2005

Hansford

1961

Hansford

1961

Stevens \&

Tehon 1926

Ciferri 1954

Hansford

1961

Hansford

1961

Mibey \&

Cannon 1999

Hansford

1954

Song \& Li

2004a

Hansford

1961

Hansford

1944

Song et al.

1997e

Stevens 1925

Hansford

1961

Spegazzini

1912 
Croton alienus

Croton caudatus

Croton curranii

Croton curranii

Croton floribundus

Croton gossypiifolius

Croton linearis (Croton angustatus)

Croton macrostachyus

Croton macrostachyus

Croton malabaricus

Croton malabaricus

Croton nigritanus

Croton sp.

Croton sp.

Croton sp.

Croton sp.

Dalechampia bidentata

Dalechampia scandens

Discoglypremna caloneura

Erythrococca rigidifolia

Euphorbia papillosa

Euphorbia pulcherrima

Euphorbia sp.

Euphorbia teke

Euphorbia tithymaloides (Pedilanthus latifolius)

Excoecaria oppositifolia var. crenulata (Excoecaria crenulata)
Meliola kerichoensis

Mibey 1998

!Asteridiella crotoniscaudati Hosag. et al. 2010

Meliola brachyodonta

Syd. 1928

Meliola brevidentata Syd. Sydow 1930 1930

Meliola longispora

(Gaillard) F. Stevens 1928

Meliola cladophaga Syd.

1926

Meliola anfracta Cif.

1938

Meliola crotonis-

macrostachydis Hansf.

1963

Meliola micropoda Hansf. Hansford

1946

1946

Asteridiella crotonicola

Hosag. \& T.K. Abraham 1998

Meliola crotonis-

malabarici Hosag. 2006

Meliola crotonis-nigritani

Deighton 1958

Irenopsis crotonis $(\mathrm{F}$.

Stevens \& Tehon) F.

Stevens 1926

Meliola crotonicola $\mathrm{F}$.

Stevens 1928

Meliola jamaicensis

Hansf. 1955

Meliola janeirensis

Hansf. 1949

Hosagoudar et

al. $1998 \mathrm{c}$

Hosagoudar

2006

Deighton

1957

Stevens \&

Tehon 1926

Stevens 1928

Hansford

1955

Hansford

1949

Asteridiella hansfordii (F. Hansford

Stevens) Hansf. $1961 \quad 1961$

\#Irenina dalechampiae F. Stevens 1927

Stevens 1927

Asteridiella entebbeensis

(Hansf. \& F. Stevens)

Hansf. 1961

Asteridiella

erythrococcae (Hansf.)

Hansf. 1961

Meliola papillosae Bat. \&

H. Maia 1965

Asteridiella chowrirae

Hosag. et al. 2011

Meliola ugandensis

Hansf. 1937

Meliola teke Hansf. 1946

Asteridiella verrucosa

(Pat.) Hansf. 1961

Meliola chandleri Hansf.

1937
Hansford

1961

Hansford

1961

Batista et al.

1965a

Hosagoudar et al. 2011 b

Hansford

1937

Hansford

1946

Hansford

1961

Hansford

1937 
Excoecaria philippinensis

Excoecaria simii (Excoecaria caffra)

Gymnanthes lucida

Hancea sp.

Hevea brasiliensis

Hippomane mancinella

Homalanthus populneus

Homonoia riparia

Homonoia riparia

Hura crepitans

Jatropha hernandiifolia

Leucocroton wrightii

Macaranga hurifolia

Macaranga peltata

Macaranga schweinfurthii

Macaranga schweinfurthii

Macaranga schweinfurthii

Macaranga sp.

Macaranga tanarius

Macaranga tanarius

Macaranga triloba

Mallotus japonicus

Mallotus oppositifolius

Mallotus paniculatus

Mallotus paniculatus

Mallotus paniculatus (Mallotus albus)
Meliola excoecariicola F. Hansford Stevens ex Hansf. 1957 1957b

Meliola excoecariae Doidge 1920c

Doidge 1920

Meliola gymnanthicola F. Stevens 1916

Stevens 1916

Asteridiella verrucosa Hansford

(Pat.) Hansf. 1961

Irenopsis vincensii D.B. Pinho et al.

Pinho \& O.L. Pereira 2014

2014

Meliola hippomaneae F. Stevens 1928

Stevens 1928

Meliola homalanthi Boedijn 1940

Boedijn 1940

Meliola homonoiae

Hosag. \& Sabeena 2011

Hosagoudar

\& Sabeena

$2011 b$

Meliola ramosii Syd. \& P. Sydow \&

Syd. 1914

Sydow 1914a

Meliola hurae Syd. 1930

Sydow 1930

Meliola jatrophae $\mathrm{F}$.

Stevens 1916

Stevens 1916

Meliola acunae Schmied. Schmiedeknec 1989

ht 1989

Meliola bougheyana S. Hughes 1952

Hughes 1952

Asteridiella

Hosagoudar

macarangicola Hosag.

1989

Asteridiella entebbeensis

(Hansf. \& F. Stevens)

Hansf. 1961

Irenopsis macarangae Hansford

Hansf. 1941

1941

Meliola macarangicola Hansford

Hansf. 1945

1945 a

Meliola apayaoensis H.S. Yates 1918

Yates 1918

Meliola brachypoda Syd. Sydow 1922a

1922

Meliola macarangae Syd. Sydow \&

\& P. Syd. 1917

Sydow 1917a

Meliola apayaoensis H.S. Yates 1918

Yates 1918

Asteridiella malloticola Hansford

(W. Yamam.) Hansf. 1961

1961

Asteridiella hansfordii (F. Hansford

Stevens) Hansf. $1961 \quad 1961$

Asteridiella malloticola Hansford

(W. Yamam.) Hansf. 1961

1961

Meliola serdangensis Hansford

Hansf. 1957

1957b

Asteridiella malloti

(Hansf. \& Thirum.)

Hansford

1961

Hansf. 1961 
Mallotus paniculatus

(Mallotus albus)

Mallotus philippensis

Mallotus philippensis

Mallotus philippensis

Mallotus philippensis

Mallotus philippensis

Mallotus philippensis

Mallotus polycarpus (Trewia polycarpa)

Mallotus resinosus

Mallotus rhamnifolius

(Croton reticulatus)

Mallotus roxburghianus

Mallotus sp.

Mallotus tetracoccus

Manihot carthaginensis subsp. Glaziovii (Manihot glaziovii)

Manihot esculenta (Manihot utilissima)

Manihot esculenta (Manihot utilissima)

Manihot palmata

Manihot palmata

Maprounea guianensis

Microstachys chamaelea

(Sebastiania chamaelea)

Omphalea pauciflora\#

Omphalea pauciflora\#

Ostodes paniculata

Paracroton pendulus (Fahrenheitia zeylanica)
Asteridiella malloti

(Hansf. \& Thirum.)

Hansford

Hansf. 1961

Appendiculella malloti B. Song et al. Song \& Y.X. Hu 1997

Asteridiella kodavae

Hosag. et al. 2013

Asteridiella kombeenisis

Hosag. 2002

Asteridiella malloti

(Hansf. \& Thirum.)

Hansf. 1961

Asteridiella subapoda

(Syd. \& P. Syd.) Hansf.

1961

Meliola malloti Srinivas.

1974

Meliola trewiae Hosag.

1991

Asteridiella resinosi

Hosag. 1996

Asteridiella crotonis

Hosag. 1989

Irenopsis hypophyllae

Jana et al. 2005

Asteridiella subapoda

(Syd. \& P. Syd.) Hansf.

1961

Asteridiella malloti

(Hansf. \& Thirum.)

Hansf. 1961

Asteridiella entebbeensis

(Hansf. \& F. Stevens)

Hansf. 1961

Asteridiella entebbeensis

(Hansf. \& F. Stevens)

Hansf. 1961

Meliola manihoticola

Henn. 1904

Meliola manihot $\mathrm{F}$.

Stevens \& Tehon 1926

Meliola manihotis F.

Stevens \& Tehon 1926

Asteridiella sapii (Hansf.)

Hansf. 1961

Asteridiella sebastianiae

Hosag. et al. 2011

Asteridiella funebris

(Cif.) Hansf. 1961

Irenopsis omphaleae

Hansf. 1955

Meliola ostodis J.N.

Kapoor 1967

Meliola fahrenheitiae

Hosag. \& T.K. Abraham 1998
$1997 d$

Hosagoudar

2013a

Hosagoudar

2002b

Hansford

1961

Hansford

1961

Srinivasulu

1974

Hosagoudar

\& Goos 1991

Hosagoudar

1996

Hosagoudar

\& Goos 1989

Jana et al.

2005a

Hansford

1961

Hansford

1961

Hansford

1961

Hansford

1961

Hennings

1904b

Stevens \&

Tehon 1926

Stevens \&

Tehon 1926

Hansford

1961

Hosagoudar

\& Sabeena

2010

Hansford

1961

Hansford

1955

Index

Fungorum

2016

Hosagoudar et al. $1998 \mathrm{c}$ 


Ricinodendron heudelotii
subsp. Africanum
(Ricinodendron africanum)
Sapium sp.
Sebastiania sp.
$\begin{aligned} & \text { Shirakiopsis elliptica (Sapium } \\ & \text { ellipticum) }\end{aligned}$

Tetrorchidium didymostemon

Tetrorchidium rubrivenium

Tragia sp.

Tragia sp.

Fabaceae
Meliola janeirensis

Hansf. 1949

Asteridiella sapii (Hansf.) Hansford

Hansf. 1961

Meliola patella Theiss.

1910

Asteridiella entebbeensis Hansford

(Hansf. \& F. Stevens)

Hansf. 1961

Amazonia tetrorchidii

Hansf. 1948

Meliola tetrorchidiicola

Hansf. 1957

Asteridiella tragiae

Hosag. \& C. Jagath

Timmaih 2013

Meliola tragiae Hosag. \&

C. Jagath Timmaih 2013

Amazonia leguminosarum

Bat. et al. 1965

Asteridiella usteri

(Hansf.) Hansf. 1963

Irenopsis trompilliana

Toro 1934

Meliola castanha Theiss.

1914

Meliola conica F. Stevens

1928

Meliola constipata

(Speg.) Speg. 1924

Meliola forsteroniae (F.

Stevens) Hansf. 1949

Meliola franciscana

Hansf. 1949

Meliola hendrickxiana

Hansf. 1947

Meliola mimosacearum

Hansf. 1955

Meliola motatanensis

Hansf. 1957

Meliola polyodonta Syd.

1926

Meliola sydowii Hansf.

1949

Meliola abri Hosag. \&

Riju 2011

Irenopsis berggrenii

Hansf. 1955

Meliola thailandicum

Hongsanan \& K.D. Hyde 2015

Meliola acaciae-confusae

Sawada 1931

Meliola brisbanensis

Hansf. 1953

Meliola acaciicola Hansf.

1963
Hansford

1949

1961

Theissen 1910

Hans
1961

Hansford \&

Deighton

1948

Hansford

$1956 b$

Hosagoudar

2013b

Hosagoudar

2013b

Batista et al.

1965b

Hansford

1962

Chardón \&

Toro 1934

Theissen 1914

Stevens 1928

Spegazzini

1924

Hansford

1949

Hansford

1949

Hansford

1947b

Hansford

1955

Hansford

1956b

Sydow 1926

Hansford

1949

Hosagoudar

\& Riju 2011a

Hansford

1953b

Hongsanan et

al. 2015

Sawada 1931

Hansford

1953a

Hansford

1962 
Acacia koa

Acacia melanoxylon

Acacia pedicellata

Acacia riparia

Adenanthera microsperma

Adenanthera pavonina

Aganope heptaphylla (Derris diadelpha)

Aganope leucobotrya

(Ostryoderris leucobotrya)

Aganope thyrsiflora

Albizia adoratissima\#

Albizia gummifera

Albizia inundata (Albizia polyantha)

Albizia niopoides (Pithecolobium hassleri) Albizia odoratissima

Albizia zygia

Anadenanthera peregrina (Piptadenia peregrina) Anadenanthera peregrina (Piptadenia peregrina)

Andira humilis

Andira inermis

Andira sp.

Archidendron bigeminum (Abarema bigemina)

Archidendron lucidum (Pithecellobium lucidum)

Baphia nitida
Meliola koae F. Stevens 1925

Meliola melanoxylonis

Hosag. \& C.M. Pillai

1994

Meliola acaciarum Speg. 1922

Amazonia acaciae Gonz.

Frag. \& Cif. 1928

Meliola adenantherae

(Cif.) Hansf. 1961

Meliola adenanthericola

Hosag. et al. 2001

Meliola abrupta Syd. \&

P. Syd. 1917

Meliola ostryoderridis

Hansf. \& Deighton 1948

Meliola aganopes Hosag. et al. 2010

Meliola odoratissimae

(J.N. Kapoor) Hosag.

1996

Meliola albiziae Hansf. \& Hansford \&

Deighton 1948

Meliola albiziae-

polyanthae W.R.O.

Soares \& Dianese 2013

Meliola pithecellobiicola

Speg. 1924

Meliola johnstonii Hansf. 1957

Meliola albiziae Hansf. \& Hansford \&

Deighton 1948

Deighton

1948

!Meliola piptadeniae Cif. Ciferri 1954 1954

Asteridiella

piptadeniicola Hansf.

1961

Meliola andirae-humilis Soares \&

W.R.O. Soares \& Dianese Dianese 2014

2013

Meliola andirae Earle Earle 1904 1905

Meliola andirae Earle $\quad$ Earle 1904

1905

Amazonia abaremae Hosag. \& V.T. Antony 1988

Asteridiella pithecellobii

(W. Yamam.) Hansf.

1961

Meliola baphiae-nitidae

Hansf. \& Deighton 1948
Hansford

1961

Hosagoudar

\& Antony

1988

Hansford

1961

Hansford \&

Deighton

1948 
Baphia polygalacea

Baphia racemosa

Baphia racemosa

Baubinia sp.

Bauhinia championii

Bauhinia glabra (Bauhinia cumanensis)

Bauhinia phoenicea

Bauhinia purpurea

Bauhinia sp.

Bauhinia sp.

Bauhinia sp.

Bauhinia sp.

Bauhinia sp.

Berlinia grandiflora (Berlinia heudelotiana)

Brya ebenus

Butea monosperma

Caesalpinia crista

(Caesalpinia nuga)

Caesalpinia crista

(Caesalpinia nuga)

Caesalpinia sappan

Caesalpinia sappan

Caesalpinia sp.

Cajanus lineatus (Atylosia lineata)

Calopogonium mucunoides

Calopogonium mucunoides

Calopogonium mucunoides (Calopogonium orthocarpum)
Meliola baphiaepolygalaceae Hansf. \& Deighton 1948

Meliola baphiae-nitidae Hansf. \& Deighton 1948

Meliola indigofera Syd. 1928

Meliola bauhiniae H.S.

Yates 1918

Meliola bauhiniicola W.

Yamam. 1941

Meliola schizolobii Syd. \& P. Syd. 1916

Meliola bauhiniae-

phoeniceae Hosag. et al. 2004

Meliola bauhiniicola W.

Yamam. 1941

Meliola burgosensis

Hansf. 1955

Meliola caesalpiniae

(Hansf. \& Deighton)

Hansf. \& Deighton 1958

Meliola

kulathupuzhaensis Hosag.

\& J.P. Jacob 2006

Meliola pazschkeana

Gaillard 1892

Meliola theissenii Hansf. Hansford 1957

$1956 \mathrm{~b}$

Meliola berliniae Hansf.

\& Deighton 1948

Meliola bryae Hansf.

1957

Meliola buteae Hafiz

Khan et al. 1955

Meliola caesalpiniicola

Deighton 1958

Hansford \&

Deighton

1948

Hansford

$1956 \mathrm{~b}$

Hafiz Khan et

al. 1955

Deighton

1958

Meliola cylindrophora

Rehm 1913

Meliola aethiops Sacc.

1921

Meliola caesalpiniae

(Hansf. \& Deighton)

Hansf. \& Deighton 1958

Meliola subtortuosa

Rehm 1901

Meliola atylosiae Hosag. Hosagoudar 1990

!Meliola calopogonii

Hosag. \& Manojk. 2005

Meliola calopogonii $\mathrm{F}$.

Stevens 1928

Meliola scabriseta Hansf. Hansford \&

\& Deighton 1948
Rehm 1913c

Saccardo

1921

Deighton

1957

Rehm 1901 \& Goos 1990a

Hosagoudar

2005

Stevens 1928

Deighton

1948 
Canavalia mattogrossensis

(Canavalia lasiocalyx)

Canavalia sp.

Cassia ferruginea

Cassia fistula

Cassia fistula

Cassia hoffmannseggii\#

Cassia sp.

Cassia sp.

Cassia sp.

Cathormion altissimum

Cathormion altissimum

Centrosema virginianum (Centrosema virginiana) Chamaecrista glandulosa

Chamaecrista glandulosa var. picardae (Cassia pinetorum)

Chamaecrista lineata var. brachyloba (Chamaecrista granulata)

Chamaecrista pedicellaris (Cassia pedicellaris)

Clitoria ternatea

Codariocalyx motorius

(Desmodium gyrans)

Codariocalyx motorius

(Desmodium motorium)

Copaifera officinalis

Crotalaria micans (Crotalaria anagyroides)

Dalbergia berteroi

Dalbergia brownei

(Amerimnon brownii)

Dalbergia sp.

Dalbergia sp.

Dalbergiella welwitschii
Meliola vignae-gracilis

Hansf. \& Deighton 1948

Meliola canavaliae

Hosag. \& Riju 2011

Meliola cassiae-

ferrugineae Pinho \& O.L.

Pereira 2012

Meliola aethiops Sacc.

1921

Meliola cassiae-fistulae

Hosag. \& Manojk. 2004

Meliola

hoffmannseggiana Hansf.

1961

\#Irene cubitella $\mathrm{F}$.

Stevens \& Tehon 1926

Asteridiella arachnoidea

(Speg.) Hansf. 1961

Meliola austro-americana

J.L. Crane \& A.G. Jones 2001

Irenopsis cathormii

Hansf. \& Deighton 1948

Irenopsis cathormionis

Hansf. \& Deighton 1948

Meliola denticulata $\mathrm{G}$.

Winter 1892

Meliola chamaecristae

Earle 1905

Meliola gliricidiae Syd. \&

P. Syd. 1914

Irenopsis

chamaecristicola $(\mathrm{F}$.

Stevens) F. Stevens 1927

Asteridiella cassiae (Cif.)

Hansf. 1961

Meliola clitoriae Hosag.

\& Goos 1990

Meliola bantamensis

Hansf. 1954

Meliola desmodii-motorii

Hosag. \& Manojk. 2005

Meliola hexaseptata Bat.

\& R. Garnier 1965

Meliola desmodii-laxiflori

Deighton 1958

Meliola bonetii Cif. 1954

Meliola amerimni (F.

Stevens) Hansf. 1961

Meliola dalbergiae Hansf.

1958

Meliola scabriseta Hansf.

\& Deighton 1948

Hansford \&

Deighton

1948

Hosagoudar

\& Riju 2011a

Pinho et al.

2012

Saccardo

1921

Hosagoudar

2004c

Hansford

1961

Stevens \&

Tehon 1926

Hansford

1961

Crane \&

Jones 2001

Hansford \&

Deighton

1948

Hansford \&

Deighton

1948

Gaillard 1892

Earle 1904

Sydow \&

Sydow 1914a

Stevens 1927

Hansford

1961

Hosagoudar

\& Goos 1990a

Hansford

1954

Hosagoudar

2005

Batista et al.

1965a

Deighton

1957

Ciferri 1954

Hansford

1961

Hansford

1957c

Hansford \&

Deighton

1948

Meliola scabriseta Hansf. Hansford \& \& Deighton 1948
Deighton

1948 
Daniellia thurifera

Delonix regia

Delonix regia

Dendrolobium triangulare

(Desmodium triangulare)

Dendrolobium triangulare

(Desmodium triangulare)

Derris sp.

Desmodium heterocarpon

Desmodium incanum

Desmodium incanum

Desmodium incanum

(Desmodium canum)

Desmodium incanum

(Desmodium supinum)

Desmodium incanum

(Meibomia cana)

Desmodium laxiflorum

Desmodium oojeinense

(Ougeinia oojeinensis)

Desmodium salicifolium

Desmodium salicifolium

Desmodium sp.

Desmodium sp.

Desmodium sp.

Desmodium sp.

Desmodium velutinum

Desmodium zonatum

Detarium senegalense

Dimorphandra conjugata

(Dimorphandra latifolia)

Dimorphandra sp.
Meliola danielliae Hansf. Hansford \&

\& Deighton 1948 Deighton

1948

Irenopsis trompilliana

Chardón \&

Toro 1934

Toro 1934

Meliola aethiops Sacc. Saccardo

1921

1921

!Meliola desmodii-

triangularis Gawande \&

D.K. Agarwal 2004

Meliola desmodii-

triangularis Hosag. \&

Manojk. 2004

Meliola derridis H.S.

Yates 1918

Meliola desmodii-

heterocarpi Hosag. \&

Sabeena 2013

Meliola bicornis $\mathrm{G}$.

Winter 1886

Meliola desmodii-laxiflori

Deighton 1958

Gawande et

al. 2004

Hosagoudar

$2004 c$

Yates 1918

Hosagoudar

\& Sabeena

2013a

Winter 1886

Meliola meibomiae F.

Stevens \& Tehon 1926

Meliola desmodii $\mathrm{P}$.

Karst. \& Roum. 1890

Asteridiella meibomiae

(F. Stevens) Hansf. 1961

Meliola desmodii-laxiflori

Deighton 1958

Deighton

1957

Stevens \&

Tehon 1926

Karsten \&

Roumeguère

1890

Hansford

1961

Meliola ougeiniae R.K. Verma et al.

Verma et al. $2008 \quad 2008$

Meliola kawandensis Hansford

Hansf. 1957

$1956 b$

Meliola stizolobii Hansf. Hansford \&

\& Deighton 1948 Deighton

1948

Asteridiella anastomosans Hansford

(G. Winter) Hansf. 1961

Meliola desmodiicola

1961

Beeli 1920

Meliola heterocephala

Syd. \& P. Syd. 1916

Meliola zollingeri

Gaillard 1892

Beeli 1920

Sydow \&

Sydow $1916 b$

Index

Fungorum

2016

Meliola desmodii-velutini

Hosag. \& Manojk. 2004

Meliola bantamensis

Hosagoudar

2004b

Hansford

1954

Meliola berliniae Hansf.

\& Deighton 1948

Hansford \&

Deighton

1948

Asteridiella cubitorum (F. Hansford Stevens \& Tehon) Hansf. 1961

1961

Irenopsis dimorphandrae Hansf. 1955
Hansford

1955 
?Dioclea sp.

Diphysa robinioides\#

Dipteryx odorata

Distemonanthus

benthamianus

Dolichos trilobus

Endosamara racemosa

(Millettia racemosa)

Entada gigas

Entada phaseoloides

Entada rheedii (Entada

pursaetha)

Eriosema congestum

Eriosema glomeratum

Erythrina poeppigiana

(Erythrina micropteryx)

Erythrina poeppigiana

(Erythrina micropteryx)

Erythrina sp.

Erythrina subumbrans

Erythrina variegata

Erythrina variegata

(Erythrina indica)

Erythrophleum guineense

Erythrophleum sp.

Euchresta horsfieldii

Feuilleea granulosa (Albizia granulosa)

Flemingia sp.

Galactia dubia

Galactia sp.

Galactia stenophylla\#
Meliola crenatissima Syd. Sydow \&

\& P. Syd. 1916

Sydow 1916a

Meliola diphysae $\mathrm{F}$.

Stevens 1928

Meliola dipterygicola Bat. Index

\& H. Maia 1966

Fungorum

2016

Meliola aethiops Sacc. Saccardo

1921

1921

Meliola dolichi Hosag. Hosagoudar

2006

Meliola millettiae-

\& Biju 2006

racemosae Hosag. \& M.

Mohanan 1996

Meliola entadae Hansf. Hansford

$1949 \quad 1949$

Meliola robinsonii Syd. Sydow 1922b

1922

Meliola entadicola Deighton

Deighton $1958 \quad 1957$

Meliola eriosemae Soares \&

W.R.O. Soares \& Dianese Dianese 2014

2013

Meliola stizolobii Hansf. Hansford \&

\& Deighton 1948 Deighton

1948

\#Meliola bicornis var. Ciferri 1933

erythrinae Cif. 1933

Meliola erythrinae- Hansford

micropterycis Hansf.

1961

Meliola tungurahuana Sydow \&

Syd. 1939

Sydow 1939

Meliola erythrinicola Deighton

Deighton 1958

1958

Meliola erythrinae Syd. \& Sydow \&

P. Syd. 1917

Sydow 1917a

Meliola erythrinae Syd. \& Sydow \&

P. Syd. 1917

Meliola erythrophlei

Hansf. \& Deighton 1948

Sydow 1917a

Hansford \&

Deighton

1948

Meliola erythrophloeicola

Mibey 1997

Mibey \&

Hawksworth

1997

Meliola euchrestae W. Index

Yamam. 1941

Fungorum

2016

Meliola albiziae-

Index

granulosae $\mathrm{B}$. Huguenin

1969

Meliola flemingiae

Bagool \& H. Biju 2004

Meliola bicornis $\mathrm{G}$.

Winter 1886

Asteridiella gesuitica

(Speg.) Hansf. 1961

Asteridiella gesuitica

(Speg.) Hansf. 1961
Fungorum

2016

Biju \&

Bagool 2004

Winter 1886

Hansford

1961

Hansford

1961 
Geissaspis cristata

Gleditsia amorphoidis

Gliricidia sepium

Gliricidia sepium

Guibourtia copallifera

(Copaifera copallifera)

Holocalyx balansae

Hylodendron gaboens

Hymenaea courbaril

Indigofera natalensis

Indigofera sp.

Indigofera tinctoria

Inga laurina

Inga semialata (Inga marginata)

Inga $\mathrm{sp}$.

Inga $\mathrm{sp.}$

Inga $\mathrm{sp}$.

Inocarpus sp.

Kingiodendron pinnatum

Leptoderris trifoliolata

Lonchocarpus bussei

Lonchocarpus domingensis

Lonchocarpus sp.

Lonchocarpus sp.

Meibomia sp.

Meibomia sp.

Meibomia sp.
Meliola geissaspidis

Hosag. \& Manojk. 2005

Meliola gleditschiae

Speg. 1912

Meliola gliricidiae Syd. \& P. Syd. 1914

Meliola gliricidiicola

Hosag. \& D.K. Agarwal 2003

Meliola copaiferae Hansf. Hansford \& \& Deighton 1948

Deighton

1948

Meliola holocalicis Speg. Spegazzini 1924

Meliola hylodendri J.M.

Yen \& Sulmont 1973

\#Meliola hymenaeicola

Gonz. Frag. \& Cif. 1927

Meliola indigofera Syd.

1928

Meliola nyanzae Hansf.

1938

Meliola tinctoriae Mibey 1997

1924

Index

Fungorum

2016

Index

Fungorum

2016

Doidge \&

Sydow 1928

Hansford

1938

Mibey \&

Hawksworth

1997

Irenopsis toruloidea $(\mathrm{F}$.

Stevens) F. Stevens 1927

Asteridiella ingicola

(Speg.) Hansf. 1961

Irenopsis ingae $(\mathrm{F}$.

Stevens \& Tehon) F.

Stevens 1927

Meliola chagres $\mathrm{F}$.

Stevens 1928

Meliola oteroana Hansf.

1957

Meliola inocarpi $\mathrm{F}$.

Stevens 1928

Meliola kingiodendri

Hosag. et al. 1993

Meliola koriensis

Deighton 1958

Meliola bussei Mibey 1997

Meliola lonchocarpicola F. Stevens 1928

Meliola bicornis $\mathrm{G}$.

Winter 1886

Meliola juruana Henn.

1904

Meliola meibomiae $\mathrm{F}$.

Stevens \& Tehon 1926

Meliola trinidadensis $\mathrm{F}$.

Stevens \& Tehon 1926

Meliola vignae-gracilis

Hansf. \& Deighton 1948
Stevens 1927

Hansford

1961

Stevens 1927

Stevens 1928

Hansford

$1956 b$

Stevens 1928

Hosagoudar et

al. 1993

Deighton

1957

Mibey \&

Hawksworth

1997

Stevens 1928

Winter 1886

Hennings

$1904 b$

Stevens \&

Tehon 1926

Stevens \&

Tehon 1926

Hansford \&

Deighton

1948 
Mezoneurum benthamianum*

Millettia chrysophylla

Millettia peguensis

Millettia rhodantha

Millettia rubiginosa

Millettia rubiginosa

Millettia rubiginosa

Millettia sanagana

Millettia sericea

Millettia sp.

Millettia splendens

Mimosa caesalpiniifolia (Mimosa caesalpiniaefolia)

Mimosa ceratonia

Mimosa invisa

Mimosa invisa var. inermis*

Mimosa procurrens

Moullava spicata

Mucuna acuminata

Mucuna imbricata

Mucuna pruriens

Mucuna pruriens

Mucuna pruriens var. hirsuta (Mucuna hirsuta)

Mucuna pruriens (Stizolobium aterrimum)

Mucuna sloanei

Mucuna sloanei
Meliola mezoneuri Hansf. Hansford \& \& Deighton 1948 Deighton 1948 Deighton $\begin{array}{ll}\text { Meliola millettiae- } & \text { Deig } \\ \text { chrysophyllae Deighton } & 1951\end{array}$ 1951

Meliola pequensis Hosag. Hosagoudar et et al. 1998

Meliola millettiaerhodanthae Hansf. \& Deighton 1948

Asteridiella millettiae Hosag. et al. 1999 al. 1998a Hansford \& Deighton 1948 Hosagoudar et al. 1999a Meliola bataanensis Syd. Sydow \& \& P. Syd. 1914 Sydow 1914a Meliola teramni (Sacc.) $\quad$ Sydow \& Syd. \& P. Syd. 1917

Meliola millettiaesanaganae Hansf. \& Deighton 1948 Sydow 1917a Hansford \& Deighton 1948 Meliola ostryoderridis Hansford \& Hansf. \& Deighton 1948 Deighton 1948 Meliola bicornis G. Winter 1886 Winter 1886

Meliola millettiaechrysophyllae Deighton 1951

Irenopsis berggrenii Hansford Hansf. 1955 $1953 b$ Meliola monochroma Cif. Ciferri 1956 1957

Meliola venezuelana

Orejuela 1944 Garcés 1944

Meliola invisiae Hosag. \& Hosagoudar et H. Biju 2001 al. 2001a Meliola mimosicola Speg. Spegazzini 1924

1924

Meliola aethiops Sacc. 1921

Meliola mucunaeacuminatae Hansf. 1954

Meliola mucunaeimbricatae Jana et al. 2005

Meliola mucunaeacuminatae Hansf. 1954 Meliola mucunicola Hongsanan \& K.D. Hyde 2015

Saccardo 1921 Hansford 1954 Jana et al. $2005 d$

Hansford 1954 Hongsanan et al. 2015

Meliola mucunae Hansf. 1948

Hansford \& Deighton 1948

Meliola stizolobii Hansf. Hansford \& \& Deighton $1948 \quad$ Deighton 1948 Asteridiella meibomiae Hansford (F. Stevens) Hansf. $1961 \quad 1961$ Meliola meibomiicola Cif. Ciferri 1954 1954 
Mucuna urens

Myroxylon peruiferum

Neorudolphia volubilis

(Rudolphia volubilis)

Newtonia elliotii (Piptadenia elliotii)

Ormocarpum verrucosum

Ormosia formosana

Pentaclethra sp.

Pentaclethra sp.

Phanera sp.

Phaseolus sp.

?Phaseolus sp.

Philenoptera cyanescens (Lonchocarpus cyanescens)

Phyllodium pulchellum

(Desmodium pulchellum)

Physostigma venenosum

Pictetia sulcata (Pictetia spinifolia)

Pithecellobium diversifolium

Pithecellobium keyense

(Pithecellobium

guadalupense)

Platysepalum sp.

Pongamia pinnata

Pseudarthria hookeri

Pseudarthria viscida

Psophocarpus tetragonolobus

Psophocarpus tetragonolobus
Meliola mисunae Hansf. 1948

Hansford \&

Deighton

1948

Meliola peruiferi D.B. Pinho et al.

Pinho et al. $2012 \quad 2012$

Meliola rudolphiae F. Stevens 1916

Stevens 1916

Meliola piptadeniae

Hansf. \& Deighton 1948

Hansford \&

Deighton

1948

Meliola ormocarpi Hansf. Hansford \&

\& Deighton 1948

Deighton

1948

Meliola ormosiae C.C. Index

Chen 1967 Fungorum

2016

Asteridiella pentaclethrae Hansford

Hansf. 1957

$1956 \mathrm{~b}$

Meliola conigera $\mathrm{F}$.

Stevens \& Tehon 1926

Meliola curviseta Racib.

1900

Stevens \&

Tehon 1926

Index

Fungorum

2016

Meliola cristata Syd.

1928

Meliola pellucida Gaillard 1892

Stevens 1928

Meliola carvalhoi

Deighton 1951

Meliola desmodii-

pulchelli Hosag. et al.

1999

Meliola physostigmatis Hansford \&

Hansf. \& Deighton 1948

Meliola pictetiae Hansf.

1955

Index

Fungorum

2016

Deighton

1951

Hosagoudar et

al. 1999 a

Deighton

1948

Hansford

1955

Meliola pithecellobii $\mathrm{F}$.

Stevens \& Tehon 1926

Stevens \&

Tehon 1926

Meliola venezuelana

Orejuela 1944

Garcés 1944

Meliola platysepali

Hansf. \& Deighton 1948

Hansford \&

Deighton

1948

Meliola pongamiae

Hosagoudar

Hosag. \& T.K. Abraham \& Abraham 1999

$1999 b$

Meliola hookeri Mibey 1997

Mibey \&

Hawksworth

1997

Meliola pseudarthriae Hosagoudar

Hosag. \& Manojk. 2004 2004b

Meliola erythrinae Syd. \& Sydow \&

P. Syd. 1917

Sydow 1917a

Meliola psophocarpi

Hosag. \& Riju 2010
Hosagoudar

\& Riju 2010 
Psoralea sp.

Pterocarpus indicus

Pterocarpus santalinoides

Pueraria sp.

Pueraria tuberosa

Pycnospora lutescens

Schizolobium parahyba (Schizolobium excelsum) Sclerolobium $\mathrm{sp}$.

Senna bacillaris (Cassia bacillaris)

Senna bacillaris (Cassia bacillaris)

Senna quinquangulata

(Cassia quinquangulata)

Senna siamea (Cassia siamea)

Senna siamea (Cassia siamea)

Senna surattensis (Cassia surattensis)

Sophora tomentosa

Spatholobus parviflorus

Spatholobus parviflorus

Sweetia fruticosa

Tadehagi triquetrum

(Desmodium triquetrum)

Tadehagi triquetrum

(Desmodium triquetrum)

Tamarindus indica

Tephrosia elegans

Tephrosia sinapou (Tephrosia toxicaria)

Teramnus labialis

Teramnus labialis

Teramnus uncinatus
Asteridiella

pseudanastomosans

(Rehm) Hansf. 1961

Hansford

1961

Meliola pterocarpi H.S.

Yates 1918

Meliola pterocarpicola Hansford \&

Hansf. \& Deighton 1948

Meliola banesensis Syd. \& P. Syd. 1916

Meliola banesensis Syd.

\& P. Syd. 1916

Meliola pycnosporae

Hosag. \& Archana 2009

Meliola schizolobii Syd.

\& P. Syd. 1916

Meliola sclerolobii Hansf.

1955

Asteridiella cassiicola

(Bat. \& J. Silva) Hansf.

1961

Meliola cassiifolii Bat. Batista et al.

1960

1960

Irenopsis toruloidea $(\mathrm{F}$.

Stevens) F. Stevens 1927

Meliola aethiops Sacc.

1921

Deighton

1948

Sydow \&

Sydow $1916 b$

Sydow \&

Sydow 1916b

Hosagoudar

\& Archana

2009

Sydow \&

Sydow 1916a

Hansford

1955

Hansford

1961

Stevens 1927

Saccardo

1921

Ciferri 1951

1951

Meliola surattensis

Hosag. et al. 1998

Hosagoudar et al. 1998 a

Meliola carbonacea Cif. 1938

!Meliola spatholobi

Hosag. et al. 2005

Ciferri 1938

Meliola banesensis Syd.

\& P. Syd. 1916

Meliola sweetiae W.R.O.

Soares \& Dianese 2013

!Meliola desmodii-

triquetri Gawande et al.

2004

Meliola desmodii-

triquetri Hosag. \&

Manojk. 2004

Meliola tamarindi Syd. \& P. Syd. 1912

Meliola stizolobii Hansf.

\& Deighton 1948

Hosagoudar

2005

Sydow \&

Sydow 1916b

Soares \&

Dianese 2014

Gawande et

al. 2004

Hosagoudar

$2004 \mathrm{c}$

Sydow \&

Sydow 1912c

Hansford \&

Deighton

1948

Meliola stizolobii Hansf.

\& Deighton 1948

Hansford \&

Deighton

1948

Meliola teramniae H.S. Yates 1917

Yates 1917

Meliola teramnicola

Hosag. et al. 2005

Biju et al.

2005

Meliola teramni (Sacc.)

Syd. \& P. Syd. 1917
Sydow \&

Sydow 1917a 
Tounatea sp.

Uraria rufescens

Vigna gracilis

Vigna khandalensis

Vigna sp.

Vigna sp.

Xylia xylocarpa

Zygia juruana (Pithecolobium jupunba)

Zygia longifolia

(Pithecellobium ligustrinum)

Fagaceae

Castanopsis armata

Castanopsis armata

Castanopsis armata

Castanopsis armata

Castanopsis carlesii

(Castanopsis stipitata)

Castanopsis carlesii

(Castanopsis stipitata)

Castanopsis faberi

Castanopsis fargesii

(Castanopsis taiwaniana)

Castanopsis formosana

Castanopsis hystrix

Castanopsis indica

Castanopsis tiberana

Castanopsis tibetana

Lithocarpus amygdalifolius (Synaedrys amygdalifolia)

Lithocarpus amygdalifolius

(Synaedrys amygdalifolius)

Lithocarpus amygdalifolius (Synaedrys amygdalifolius)
Meliola tounateae $\mathrm{F}$.

Stevens 1928

Stevens 1928

Meliola urariae Hosag. Hosagoudar

2004

2004b

Meliola vignae-gracilis

Hansford \&

Hansf. \& Deighton 1948

Deighton

1948

Meliola phaseoli Thite ex Hosagoudar

Hosag. 1996

1996

Meliola bodoquenensis

Soares \&

W.R.O. Soares \& Dianese

2013

Meliola paraphialis

Mibey 1997

Dianese 2014

Mibey \&

Hawksworth

1997

Asteridiella xyliae Hosag. Hosagoudar

2013

$2013 b$

Meliola pithecellobii $\mathrm{F}$.

Stevens \& Tehon 1926

Stevens \&

Meliola venezuelana

Garcés 1944

Amazonia balakrishnanii

Hosag. 1990

Tehon 1926

Orejuela 1944

Hosagoudar

\& Goos

$1990 \mathrm{~b}$

Asteridiella phukanea

Hosag. et al. 2009

Hosagoudar et

al. 2009d

Hosagoudar

\& Goos

$1990 b$

Meliola meghalayensis

Hosag. et al. 2009

Hosagoudar et

al. 2009d

Yamamoto

1941c

Yamam. 1941

Reynolds

$\begin{array}{ll}\text { Setameliola fusispora (W. } & \text { Reyno } \\ \text { Yamam.) D.R. Reynolds } & 2010\end{array}$

2010

Meliola fabri B. Song et

al. 2003

Song et al.

2003

Meliola taiwaniana W.

Yamam. 1941

Yamamoto

1941c

Meliola castanopsina W.

Yamam. 1941

Yamamoto

$1941 c$

Meliola hystricis A.K.

Kar \& Maity 1970

Kar \& Maity

$1970 b$

Meliola castanopsidicola

J.L. Crane \& A.G. Jones

Crane \&

Jones 2001

2001

Meliola castanopsidis Hansford

Hansf. 1948

1948

Asteridiella castanopsidis

(Hansf.) Hansf. 1961

Hansford

1961

Meliola subpellucida W. Yamamoto

Yamam. 1941

$1941 \mathrm{c}$

Appendiculella

Hansford

1961

Yamam.) Hansf. 1961

Appendiculella

lithocarpicola (W.

Hansford

1961

Yamam.) Hansf. 1961 
Lithocarpus brevicaudatus

(Synaedrys brevicaudata)

Lithocarpus brevicaudatus

(Synaedrys brevicaudata)

Lithocarpus corneus

(Synaedrys kodaihoensis)

Lithocarpus hancei

(Cyclobalanopsis

ternaticupula)

Lithocarpus hancei

(Cyclobalanopsis

ternaticupula)

Lithocarpus kawakamii

(Synaedrys kawakamii)

Lithocarpus konishii

(Synaedrys konishii)

Lithocarpus lepidocarpus

(Synaedrys lepidocarpa)

Lithocarpus ovalis (Quercus

ovalis)

Lithocarpus shinsuiensis

(Synaedrys shinsuiensis)

Lithocarpus sp.

Lithocarpus sp.

Quercus angustinii\#

Quercus glauca

(Cyclobalanopsis glauca)

Quercus glauca

(Cyclobalanopsis glauca)

Quercus languinosa*

Quercus nigra

Quercus oblongata (Quercus

leucotrichophora)

Quercus obtusata (Quercus

spicata)

Quercus oocarpa

Quercus sp.

Quercus velutina

Flacourtiaceae

Garryaceae

Aucuba japonica

Garrya sp.

Gentianaceae Anthocleista procera (Anthocleista frezoulsii)
Meliola lithocarpina $\mathrm{W}$.

Yamam. 1941

Meliola ramulicola $\mathrm{W}$.

Yamam. 1941

Meliola kodaihoensis W.

Yamam. 1941

Appendiculella

arisanensis (W. Yamam.)

Hansf. 1961

Asteridiella

cyclobalanopsidicola (W.

Yamam.) Hansf. 1961

Meliola kawakamii W.

Yamam. 1941

Appendiculella konishii

(W. Yamam.) Hansf.

1961

Meliola kiraiensis W.

Yamam. 1941

Asteridiella quercina

(Hansf.) Hansf. 1961

Appendiculella

sinsuiensis (W. Yamam.)

Hansf. 1961

Meliola cyclobalanopsina

W. Yamam. 1941

Yamamoto

$1941 c$

Yamamoto

1941c

Yamamoto

1941c

Hansford

1961

Hansford

1961

Yamamoto

1941c

Hansford

1961

Yamamoto

$1941 \mathrm{c}$

Hansford

1961

Hansford

1961

Index

Fungorum

2016

Meliola fagacearum B. Song et al.

Song \& Goos 2002

$2002 \mathrm{a}$

Meliola quercicola $\mathrm{H} . \mathrm{Hu} \quad \mathrm{Hu} 1991 \mathrm{c}$

1991

Meliola cyclobalanopsina

W. Yamam. 1941

Index

Fungorum

2016

Meliola taityuensis $\mathrm{W}$.

Yamam. 1941

Yamamoto

$1941 \mathrm{c}$

Appendiculella

himalayana R.K.

Chaudhary et al. 1994

Irenopsis quercifolia

Hansf. 1955

Chaudhary et

al. 1994

Hansford

1955

Meliola bosei Hosag.

1996

Hosagoudar

1996

Meliola melanochaeta

Syd. 1928

Sydow 1928

Irenopsis costaricensis F. Stevens 1927

Stevens 1927

Asteridiella quercina

(Hansf.) Hansf. 1961

Hansford

1961

Appendiculella quercina

Jana \& S.N. Ghosh 2008

Jana \& Ghosh

2008a

Hosagoudar

Asteridiella chukrasiae

Hosag. 2006

Asteridiella aucubae

(Henn.) Hansf. 1961

\& Biju 2006

Hansford

1961

Meliola garryae Hansf.

1955

Hansford

1955

Asteridiella anthocleistae

(Hansf. \& Deighton)

Hansford

1961

Hansf. 1961 
Anthocleista sp.

Bisgoeppertia scandens

Chelonanthus acutangulus*

Chelonanthus grandiflorus

(Lisianthius grandiflorus)

Exacum sp.

Exacum tetragonum

Fagraea ceilanica

Fagraea fragrans

Fagraea plumeriifolia

Lisianthius laxiflorus

Gesneriaceae

Besleria longipes
Besleria umbrosa

Boea pseudoglandulosa\#

Columnea grata (Columnea heterophylla)

Cyrtandra cordifolia

Cyrtandra lessoniana

Epithema sp.

Fieldia australis

Gesneria pedunculosa

(Gesneria albiflora)

Henckelia humboldtiana

(Didymocarpus

humboldtianus)

Gnetum gnemon

Gnetum montanum

Scaevola chamissoniana

Scaevola frutescens*

Scaevola sp.

Exbucklandia populnea

(Bucklandia populnea)

Exbucklandia populnea

(Symingtonia populnea)

Loropetalum chinense
Asteridiella trachylaena

(Syd.) Hansf. 1961

Hansford

1961

Meliola bisgoeppertiae

(Cif.) Cif. 1961

Meliola chelonanthi

Hansf. 1957

Meliola lisianthi $\mathrm{F}$.

Stevens \& Tehon 1926

Meliola exacigena Hosag. et al. 2007

Ciferri 1961

Meliola exaci Hosag.

1996

Asteridiella fagraeae

Hosag. \& A. Sabeena

2013

Meliola brooksii Hansf. Hansford

1955

1955

Meliola fagraeae Syd. \&

P. Syd. 1914

Meliola lisianthicola

Hansf. 1957

Hansford

$1957 b$

Stevens \&

Tehon 1926

Hosagoudar et

al. $2007 \mathrm{c}$

Hosagoudar

1996

Hosagoudar

$2013 b$

Meliola besleriae Hansf.

1955

Sydow \&

Sydow 1914a

Hansford

$1956 b$

Hansford

Meliola wismarensis F. $\quad$ Stevens 1928

Stevens 1928

Meliola pumila Syd. 1925 Sydow 1925

Meliola columneae F. Stevens 1928

Stevens 1928

Asteridiella cyrtandrae Hansford

(F. Stevens) Hansf. 1961

Asteridiella cyrtandrae Hansford

(F. Stevens) Hansf. 19611961

Meliola epithematis F. Hansford

Stevens \& Roldan ex 1962

Hansf. 1963

Irenopsis fieldiae Hansf. Hansford 1953

1953 a

Meliola gesneriae F. $\quad$ Stevens 1916

Stevens 1918

Asteridiella cyrtandrae Hansford

(F. Stevens) Hansf. 19611961

Meliola gneti Hansf. 1954 Hansford

1954

Meliola gneticola Y.X. $\quad$ Hu et al. 1992

$\mathrm{Hu} 1992$

Irenopsis scaevolicola $(\mathrm{F}$. Stevens 1927

Stevens) F. Stevens 1927

Meliola scaevolae Syd. \& Sydow \&

P. Syd. 1914

Amazonia scaevolae

Sydow 1914a

Hansf. 1957

Hansford

$1957 b$

Meliola bucklandiae A.K. Kar \& Maity

Kar \& Maity 1970

$1970 b$

Meliola symingtoniae J.N. Index

Kapoor 1967

Fungorum

2016

Meliola loropetalicola

Hu et al. 1990

Y.X. Hu \& Ouyang 1990 


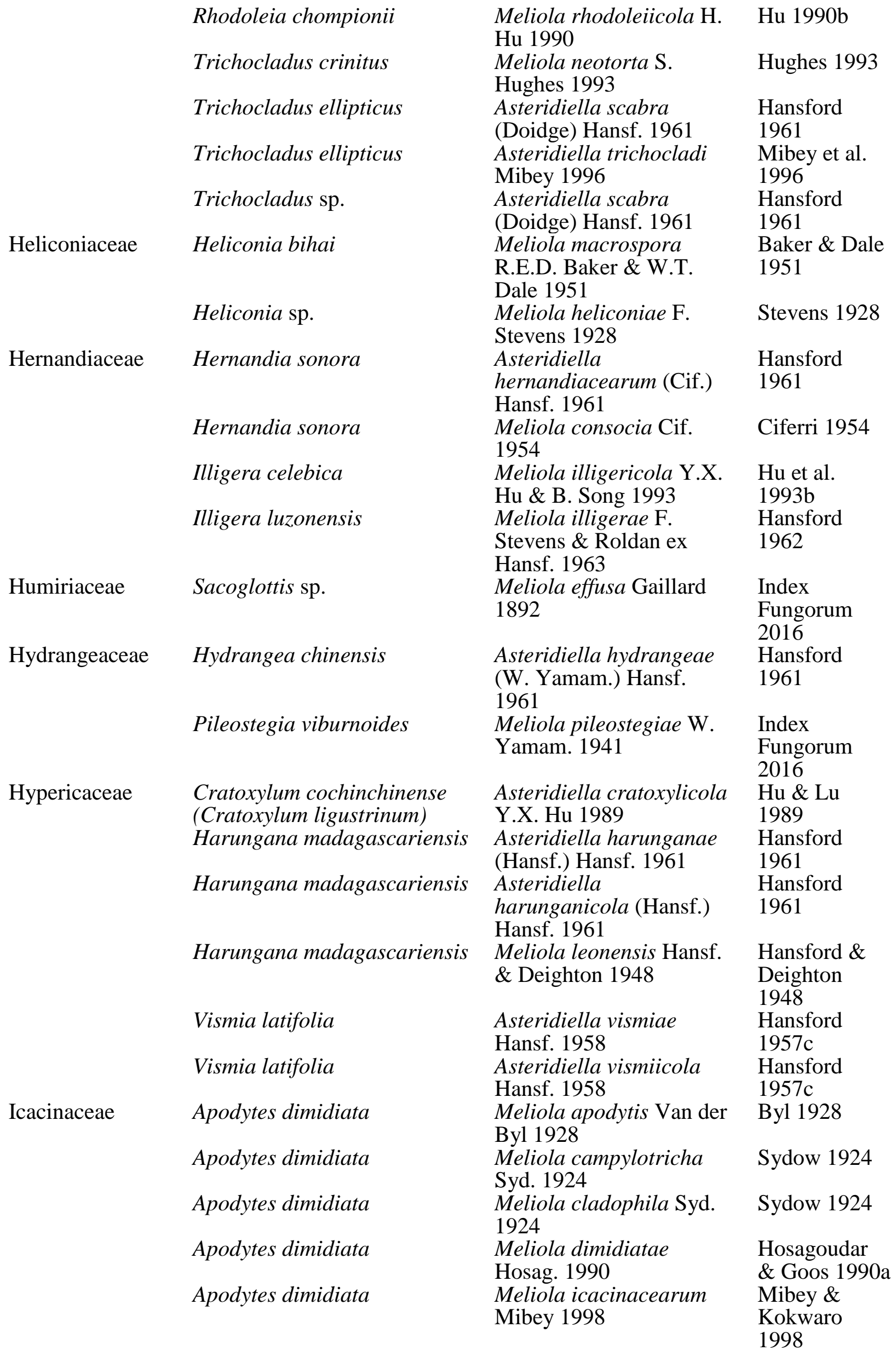


Apodytes dimidiata (Apodytes beddomei)

Mappia racemosa

Mappianthus iodoides

Pyrenacantha sp.

Sarcostigma kleinii

\section{Sarcostigma kleinii}

Irvingiaceae

Iteaceae

Ixonanthaceae

Lamiaceae
Irvingia tenuinucleata (Irvingia wombolu) Choristylis rhamnoides

Phyllocosmus africanus (Ochthocosmus africanus)

Phyllocosmus africanus (Ochthocosmus africanus)

Phyllocosmus africanus (Ochthocosmus africanus)

Engelhardtia roxburghiana

Engelhardtia roxburghiana (Engelhardtia chrysolepis)

Engelhardtia roxburghiana (Engelhardtia fenzlii)

\section{Achyrospermum oblongifolium \\ Aegiphila elata \\ Aegiphila elata}

Aegiphila elata

Aegiphila sp.

?Aegiphila sp.

Anisomeles indica

(Anisomeles ovata)

Callicarpa americana

Callicarpa arborea (Callicarpa magna)
Meliola

Hosagoudar chandrasekharanii Hosag. \& Goos 1990a 1990

Meliola mappiae Cif.

Ciferri 1931

1931

Meliola mappianthicola Yang 1989

J.C. Yang 1989

Irenopsis comata

Stevens 1927

(Doidge) F. Stevens 1927

Meliola sarcostigmaticola

Hosag. \& T.K. Abraham 1999

Meliola sarcostigmatis

Hosag. 1990

Meliola irvingae Hansf.

1947

Meliola choristylidis

Doidge 1927

Meliola linacearum

Hansf. \& Deighton 1948

Meliola ochthocosmi

Hosagoudar

\& Abraham

1999b

Hosagoudar

\& Goos 1990a

Hansford

$1947 b$

Doidge 1927

Hansford \&

Deighton

1948

Hansford \&

Hansf. \& Deighton 1948

Deighton

1948

Meliola ochthocosmicola

Hansford \&

Deighton

1948

!Appendiculella

engelhardtiicola $\mathrm{H}$. $\mathrm{Hu}$

1990

Appendiculella

engelhardtiae (W.

Yamam.) Hansf. 1961

!Asteridiella

engelhardtiicola $\mathrm{H}$. $\mathrm{Hu}$

1990

Appendiculella

Hu 1990b

labiatarum Hansf. 1961

Meliola achyrospermi

Hansf. \& Deighton 1948

Asteridiella aegiphilae

(Hansf.) Hansf. 1961

Asteridiella anthocleistae

(Hansf. \& Deighton)

Hansf. 1961

Meliola renovata $\mathrm{Cif}$.

1954

Meliola aegiphilae F.

Stevens 1927

Meliola pseudocapensis

Hansf. 1955

Meliola anisomelis Jana

et al. 2005

Meliola cookeana Speg.

1881

Hansford

1961

Hu 1990b

Hansford

1961

Hansford \&

Deighton

1948

Hansford

1961

Hansford

1961

Ciferri 1954

Hansford

1961

Hansford

1955

Jana et al.

$2005 c$

Index

Fungorum

2016

\#Irenina callicarpae F. Stevens \&

Stevens \& Roldan 1935 Roldan 1935 
Callicarpa arborea

(Callicarpa magna)

Callicarpa candicans

(Callicarpa cana)

Callicarpa candicans

(Callicarpa cana)

Callicarpa erioclona

Callicarpa formosana

Callicarpa formosana

(Callicarpa blancoi)

Callicarpa formosensis

Callicarpa longifolia

Callicarpa $\mathrm{sp.}$

Clerodendrum capitatum

Clerodendrum capitatum

Clerodendrum cyrtophyllum

Clerodendrum infortunatum

(Clerodendrum viscosum)

Clerodendrum infortunatum

(Clerodendrum viscosum)

Clerodendrum $\mathrm{sp}$.

Clerodendrum sp.

Clerodendrum sp.

Clerodendrum trichotomum

Colebrookea oppositifolia

Coleus sp.

Gmelina philippensis

Gomphostemma sp.

Hyptis atrorubens

Hyptis capitata

Hyptis lantanifolia

Hyptis pectinata

Hyptis sp.
Asteridiella callicarpae F. Hansford

Stevens \& Roldan ex $\quad 1962$

Hansf. 1963

Asteridiella vilis (Syd. \& Hansford P. Syd.) Hansf. $1961 \quad 1961$

Meliola callicarpae Syd. Sydow \&

\& P. Syd. 1912

Asteridiella vilis (Syd. \& Hansford

P. Syd.) Hansf. $1961 \quad 1961$

Meliola callicarpicola W. Index

Yamam. 1941

Fungorum

2016

Asteridiella vilis (Syd. \& Hansford

P. Syd.) Hansf. $1961 \quad 1961$

Meliola formosensis W. Yamamoto

Yamam. $1941 \quad 1941 \mathrm{a}$

Asteridiella callista Hansford

(Rehm) Hansf. $1961 \quad 1961$

Meliola arnoldii Schmied. Schmiedeknec

1989

ht 1989

Meliola capitati Mibey 1997

Mibey \&

Hawksworth

1997

Meliola clerodendricola Hennings

Henn. $1898 \quad 1898$

Meliola aegiphilae F. Hansford

Stevens $1927 \quad 1961$

Asteridiella Hosagoudar

clerodendricola Hosag. ～\& Goos 1989

1989

Asteridiella

vivekananthanii Hosag.

1988

!Meliola clerodendri W. Yamamoto

Yamam. 1940

Meliola clerodendri

Hansf. 1937

1940

Hansford

1937

Meliola durantae Gaillard

1892

Meliola sakawensis Henn. Hennings

1904

$1904 \mathrm{a}$

Asteridiella colebrookiae

Jana et al. 2005

Jana et al.

2005a

Meliola cavitensis H.S. Yates 1918

Yates 1918

Meliola micromera Syd.

\& P. Syd. 1914

Asteridiella anastomosans

(G. Winter) Hansf. 1961

Asteridiella anastomosans

(G. Winter) Hansf. 1961

Asteridiella anastomosans

(G. Winter) Hansf. 1961

Asteridiella anastomosans

(G. Winter) Hansf. 1961

Asteridiella anastomosans

(G. Winter) Hansf. 1961

Asteridiella arachnoidea

(Speg.) Hansf. 1961
Sydow \&

Sydow 1914a

Hansford

1961

Hansford

1961

Hansford

1961

Hansford

1961

Hansford

1961

Hansford

1961 


\begin{tabular}{|c|c|c|}
\hline Hyptis suaveolens & Meliola hyptidis Syd. \& & Sydow \& \\
\hline $\begin{array}{l}\text { Ocimum carnosum (Ocimum } \\
\text { selloi) }\end{array}$ & $\begin{array}{l}\text { P. Syd. } 1910 \\
\text { Meliola ocimincola Petr. }\end{array}$ & $\begin{array}{l}\text { Sydow } 1910 \\
\text { Petrak } 1962\end{array}$ \\
\hline Oxera robusta & $\begin{array}{l}\text { Meliola oxerae B. } \\
\text { Huguenin } 1969\end{array}$ & $\begin{array}{l}\text { Index } \\
\text { Fungorum } \\
2016\end{array}$ \\
\hline Petitia domingensis & $\begin{array}{l}\text { Meliola petitiae Hansf. } \\
1955\end{array}$ & $\begin{array}{l}\text { Hansford } \\
1955\end{array}$ \\
\hline Petraeovitex wolfei & $\begin{array}{l}\text { Meliola petraeoviticis } \\
\text { Deighton } 1958\end{array}$ & $\begin{array}{l}\text { Deighton } \\
1958\end{array}$ \\
\hline $\begin{array}{l}\text { Plectranthus barbatus (Coleus } \\
\text { barbatus) } \\
\text { Plectranthus ciliatus }\end{array}$ & $\begin{array}{l}\text { Asteridiella anastomosans } \\
\text { (G. Winter) Hansf. } 1961 \\
\text { \#Limacinia capnodioides } \\
\text { (Thüm.) Sacc. } 1897\end{array}$ & $\begin{array}{l}\text { Hansford } \\
1961 \\
\text { Index } \\
\text { Fungorum } \\
2016\end{array}$ \\
\hline Pogostemon heyneanus & $\begin{array}{l}\text { Meliola pogostemonis } \\
\text { Hansf. } 1957\end{array}$ & $\begin{array}{l}\text { Hansford } \\
1956 b\end{array}$ \\
\hline Pogostemon menthoides & $\begin{array}{l}\text { Asteridiella anastomosans } \\
\text { (G. Winter) Hansf. } 1961\end{array}$ & $\begin{array}{l}\text { Hansford } \\
1961\end{array}$ \\
\hline Pogostemon travancoricus & $\begin{array}{l}\text { Amazonia dikesinghii } \\
\text { Hosag. et al. } 2010\end{array}$ & $\begin{array}{l}\text { Hosagoudar et } \\
\text { al. 2010c }\end{array}$ \\
\hline Premna glaberrima & $\begin{array}{l}\text { Meliola premnicola } \\
\text { Hosag. } 1990\end{array}$ & $\begin{array}{l}\text { Hosagoudar } \\
\& \text { Goos 1990a }\end{array}$ \\
\hline Premna glaberrima & $\begin{array}{l}\text { Meliola premnigena } \\
\text { Hosag. \& Riju } 2011\end{array}$ & $\begin{array}{l}\text { Hosagoudar } \\
\text { \& Riju 2011a }\end{array}$ \\
\hline Premna hispida & $\begin{array}{l}\text { Asteridiella premnae } \\
\text { (Hansf. \& Deighton) } \\
\text { Hansf. } 1961\end{array}$ & $\begin{array}{l}\text { Hansford } \\
1961\end{array}$ \\
\hline Premna odorata & $\begin{array}{l}\text { Asteridiella callista } \\
\text { (Rehm) Hansf. } 1961\end{array}$ & $\begin{array}{l}\text { Hansford } \\
1961\end{array}$ \\
\hline Premna odorata & $\begin{array}{l}\text { Meliola premnae Hansf. } \\
1949\end{array}$ & $\begin{array}{l}\text { Hansford } \\
1949\end{array}$ \\
\hline $\begin{array}{l}\text { Premna serratifolia (Premna } \\
\text { obtusifolia) }\end{array}$ & $\begin{array}{l}\text { Asteridiella callista } \\
\text { (Rehm) Hansf. } 1961\end{array}$ & $\begin{array}{l}\text { Hansford } \\
1961\end{array}$ \\
\hline Premna sp. & $\begin{array}{l}\text { Asteridiella madikeriensis } \\
\text { Hosag. et al.. } 2011\end{array}$ & $\begin{array}{l}\text { Hosagoudar et } \\
\text { al. } 2011 \mathrm{~b}\end{array}$ \\
\hline Premna sp. & $\begin{array}{l}\text { Asteridiella premnigena } \\
\text { Hosag. } 2013\end{array}$ & $\begin{array}{l}\text { Hosagoudar } \\
2013 \mathrm{~b}\end{array}$ \\
\hline Prostanthera sieberi & $\begin{array}{l}\text { Meliola prostantherae } \\
\text { Hansf. } 1953\end{array}$ & $\begin{array}{l}\text { Hansford } \\
1953 a\end{array}$ \\
\hline Pycnostachys sp. & $\begin{array}{l}\text { Meliola pycnostachydis } \\
\text { Hansf. } 1938\end{array}$ & $\begin{array}{l}\text { Hansford } \\
1938\end{array}$ \\
\hline $\begin{array}{l}\text { Rotheca incisa } \\
\text { (Clerodendrum incisum var. } \\
\text { macrosiphom) }\end{array}$ & $\begin{array}{l}\text { Meliola aegiphilae F. } \\
\text { Stevens } 1927\end{array}$ & $\begin{array}{l}\text { Hansford } \\
1961\end{array}$ \\
\hline $\begin{array}{l}\text { Rotheca serrata } \\
\text { (Clerodendrum serratum) }\end{array}$ & $\begin{array}{l}\text { Meliola aegiphilae F. } \\
\text { Stevens } 1927\end{array}$ & $\begin{array}{l}\text { Hansford } \\
1961\end{array}$ \\
\hline $\begin{array}{l}\text { Rotheca serrata } \\
\text { (Clerodendrum serratum) }\end{array}$ & $\begin{array}{l}\text { Meliola castlerockensis } \\
\text { Srinivas. } 1974\end{array}$ & $\begin{array}{l}\text { Srinivasulu } \\
1974\end{array}$ \\
\hline Symphorema involucratum & $\begin{array}{l}\text { Meliola } \\
\text { symphorematicola Hosag. } \\
\text { et al. } 1994\end{array}$ & $\begin{array}{l}\text { Hosagoudar et } \\
\text { al. } 1994 \mathrm{c}\end{array}$ \\
\hline Symphorema luzonicum & $\begin{array}{l}\text { !Meliola symphoremae } \mathrm{F} \text {. } \\
\text { Stevens \& Roldan } 1935\end{array}$ & $\begin{array}{l}\text { Stevens \& } \\
\text { Roldan } 1935\end{array}$ \\
\hline Symphorema luzonicum & $\begin{array}{l}\text { Meliola symphorematis } \\
\text { Petr. } 1959\end{array}$ & Petrak 1958 \\
\hline Tectona grandis & $\begin{array}{l}\text { Meliola tectonae Jana et } \\
\text { al. } 2005\end{array}$ & $\begin{array}{l}\text { Jana et al. } \\
2005 \mathrm{c}\end{array}$ \\
\hline
\end{tabular}


Tetradenia sp.

Tetradenia sp.

Vitex altissima

Vitex doniana (Vitex

cienkowskii)

Vitex ferruginea

Vitex negundo

Vitex pinnata

Vitex sp.

Vitex sp.

Vitex sp.

Vitex sp.

Vitex trifolia (Vitex paniculata)

Volkameria inermis

(Clerodendrum inerme)

Lauraceae

Actinodaphne hookeri

Actinodaphne lancifolia*

Actinodaphne lancifolia*

Actinodaphne mushaensis

Actinodaphne mushaensis

Actinodaphne sp.

Actinodaphne sp.

Actinodaphne sp.
Armatella tetradeniae

Hansf. 1958

Meliola tetradeniae

(Berk.) Theiss. \& Syd.

1914

Meliola altissimae Hosag. Hosagoudar

1991

Meliola aegiphilae $\mathrm{F}$.

Stevens 1927

Meliola ferrugineae

Mibey 1997

Asteridiella viticisnegundinis Hosag. et al. 2011

Meliola viticis-pinnatae

H. Biju \& Bagool 2004

Irenopsis aciculosa (G.

Winter) F. Stevens 1927

Irenopsis viticifolii Bat. \&

Carneiro 1960

\& Goos 1991

Hansford

1961

Mibey \&

Hawksworth

1997

Hosagoudar et

al. $2011 \mathrm{~b}$

Biju \&

Bagool 2004

Stevens 1927

Index

Fungorum

2016

Meliola paraensis Henn. Hennings

1900

$1900 \mathrm{~b}$

Meliola viticicola Hansf.

1945

Hansford

$1945 b$

Asteridiella depokensis

(Hansf.) Hansf. 1963

Meliola aegiphilae $\mathrm{F}$.

Stevens 1927

Hansford

1962

Hansford

1961

Asteridiella arachnoidea Hansford

(Speg.) Hansf. 1961

1961

Asteridiella calva (Speg.)

Hansf. 1961

Hansford

Asteridiella radiata

(Henn.) Sacc. 1905

Meliola cholakadensis

Hosag. et al. 2000

Meliola praetervisa

Gaillard 1892

Amazonia actinodaphnis

Hosag. 1989

!Meliola actinodaphnes I.

Hino \& Katum. 1957

1961

Index

Fungorum

2016

Hosagoudar et

al. 2000a

Gaillard 1892

Hosagoudar

\& Goos 1989

Hino \&

Katumoto

1957

Meliola kagonoki I. Hino

\& Katum. 1963

Katumoto

1963

Appendiculella kiraiensis

(W. Yamam.) Hansf.

1961

Hansford

1961

Armatella formosana $\mathrm{W}$.

Yamam. 1957

Yamamoto

1957

Armatella actinodaphnes

Hosag. et al. 2005

Biju et al.

2005

Meliola actinodaphnes

Hansf. 1948

Hansford

1948

Meliola banasuranii

Hosag. et al. 2005
Biju et al.

2005 
Actinodaphne sp.

Actinodaphne sp.

Actinodaphne wightiana (Actinodaphne malabarica)

Actinodaphne wightiana (Actinodaphne malabarica) Apollonias arnottii

Apollonias arnottii

Apollonias sp.

Beilschmiedia erythrophloia

Beilschmiedia wightii

Caryodaphnopsis tonkinensis (Persea pyriformis)

Cassytha filiformis

Cinnamomum costaricanum (Phoebe costaricana)

Cinnamomum iners

\section{Cinnamomum iners}

Cinnamomum iners

Cinnamomum malabatrum

Cinnamomum malabatrum

Cinnamomum malabatrum

Cinnamomum neurophyllum (Phoebe neurophylla)

Cinnamomum

pseudopedunculatum

Cinnamomum riparium

Cinnamomum sp.

Cinnamomum sp.

?Cinnamomum sp.

Cinnamomum subavenium

(Cinnamomum randaiense)

Cinnamomum verum

(Cinnamomum zeylanicum)

Cryptocarya beddomei
Meliola gooseana Hosag. Hosagoudar \& T.K. Abraham 1998 \& Abraham $1998 b$

Meliola shettyi Hosag. et Hosagoudar al. 1996

1996

Meliola

Hosagoudar et actinodaphneicola Hosag. al. 1998e

\& T.K. Abraham 1998

Meliola linderae W.

Yamam. 1941

Yamamoto

1941a

Armatella apolloniadis

Hosag. et al. 2005

Biju et al.

2005

Armatella kakachiana

Hosag. \& Goos 1996

Hosagoudar

Armatella apollonigena

Hosag. \& A. Sabeena

2013

Meliola beilschmiediae

W. Yamam. 1941

\& Goos 1996

Hosagoudar et

al. 2013

Yamamoto

$1941 b$

Meliola beilschmiediicola Hosagoudar

Hosag. 1996

1996

Meliola acutiseta Syd. \&

P. Syd. 1913

Sydow \&

Sydow 1913b

Meliola cassythae Bat. et Batista et al.

al. 1965

1965a

Meliola phoebes Hansf. Hansford

1949

1949

Armatella cinnamomicola

Hansf. 1954

Hansford

1954

Armatella sumatrensis

Hansf. 1957

Hansford

$1956 b$

Meliola beilschmiediae

Yamamoto

W. Yamam. 1941

$1941 b$

Armatella balakrishnanii

Hosag. 1991

Hosagoudar

$1991 b$

Armatella indica Hosag. Hosagoudar 1991

$1991 b$

Meliola beilschmiediae

Yamamoto

W. Yamam. 1941

$1941 b$

Meliola uncitricha Syd. $\quad$ Sydow 1926

1926

Armatella litseae (Henn.)

Theiss. \& Syd. 1915

Amazonia cinnamomi

Hosag. 1988

Meliola cinnamomi

Hosag. \& T.K. Abraham 1999

Meliola kaveriappae

Hosag. et al. 1996

Meliola zigzac Berk. \&

M.A. Curtis 1868

Armatella longispora W.

Yamam. 1957

Armatella cinnamomi

Hansf. \& Thirum. 1948

Meliola kakachiana

Hosag. 1996
Theissen \&

Sydow 1915

Hosagoudar

1988 b

Hosagoudar

\& Abraham

$1999 b$

Hosagoudar

1996

Berkeley \&

Curtis 1869

Yamamoto

1957

Hansford \& Thirumalacha r 1948

Hosagoudar 1996 
Cryptocarya chinensis

Cryptocarya concinna

Cryptocarya glaucescens

(Cryptocarya meisneri)

Cryptocarya glaucescens

(Cryptocarya meisneri)

Cryptocarya latifolia

Cryptocarya mannii

Cryptocarya patentinervis*

Cryptocarya $s p$

Cryptocarya sp.

Cryptocarya sp.

Cryptocarya wightiana

(Cryptocarya bourdillonii)

Cryptocarya wightiana

(Cryptocarya bourdillonii)

Cryptocarya wightiana

(Cryptocarya bourdillonii)

Cryptocarya wightiana

(Cryptocarya bourdillonii)

Licaria triandra (Misanteca triandra)

Lindera communis

Litsea cubeba

Litsea floribunda*

Litsea floribunda*

Litsea glutinosa

Litsea insignis

Litsea insignis

Litsea mollis

Litsea perrottetii

Litsea perrottetii

Litsea $\mathrm{sp}$.

Litsea $\mathrm{sp}$.

Litsea $\mathrm{sp}$.
Meliola neolitseae W.

Yamam. 1941

Yamamoto

\#Meliola fraseriana Syd. Farr \&

1937

Rossman

2016

\#Meliola fraseriana Syd. Sydow 1937

1937

\#Meliola fraseriana Syd. Hansford 1937

$1956 \mathrm{~b}$

Meliola cryptocaryae

Doidge 1920

Asteridiella sheariana Hansford

Hansf. 1963

1962

Meliola dactylopoda Syd. Doidge \&

1928

Sydow 1928

Meliola periyakanalensis Hosagoudar

Hosag. \& B. Divya 2013

\& Divya 2013

Meliola calochaeta Syd.

1925

Meliola philippinensis (F. Hansford

Stevens) Hansf. $1961 \quad 1961$

Armatella cryptocaryae

Hosag. 1991

Meliola cryptocaryicola

Hosag. \& Raghu 1993

Meliola patileana Hosag. 1996

Meliola pushpangadanii

Hosag. \& T.K. Abraham 1998

Meliola misantecae

Hosagoudar

$1991 b$

Hosagoudar

\& Raghu

1993

Hosagoudar

1996

Hosagoudar et al. $1998 \mathrm{c}$

Hansford

$1956 b$

Yamamoto

Meliola linderae W.

Yamam. 1941

Meliola litseae-citratae

Hansf. 1954

1941a

Hansford

1954

Hosagoudar

!Meliola litseae var.

\& Goos 1990a

floribundae Hosag. 1990

!Meliola litseae var.

microspora Hosag. 1988

!Meliola litseae var.

rotundipoda Hansf. 1954

!Meliola litseae var.

insignis Hosag. 1990

Hosagoudar

1987a

Hansford

1954

Hosagoudar

\& Goos 1990a

Meliola drepanochaeta

Syd. 1926

Meliola saccardoi (Syd.

\& P. Syd.) Syd. 1922

!Meliola litseae Syd. \& P.

Syd. 1917

Sydow 1926

Sydow 1922a

Meliola saccardoi (Syd.

\& P. Syd.) Syd. 1922

!Meliola kakachiana var. poochiparaensis Hosag.

\& Sabeena 2012

!Meliola litseae f. litseae

H.S. Yates 1917

!Meliola litseae H.S

Yates 1917
Sydow \&

Sydow 1917 a

Sydow 1922a

Hosagoudar

\& Sabeena

2012

Yates 1917

Yates 1917 
Litsea sp.

Litsea sp.

Litsea $\mathrm{sp}$.

Litsea $\mathrm{sp}$.

Litsea sp.

Litsea sp.

Litsea sp.

Litsea $\mathrm{sp}$.

Litsea stocksii var.

glabrescense*

Machilus japonica var.

kusanoi (Machilus kusanoi)

Nectandra coriacea

Nectandra sp.

Nectandra sp.

Neolitsea levinei

Neolitsea sericea (Litsea glauca)

Neolitsea sp.

Nothaphoebe konishii

Ocotea lancifolia (Phoebe lanceolata)

Ocotea leucoxylon

Ocotea leucoxylon

Ocotea leucoxylon

Persea americana

Persea americana (Persea gratissima)

Persea cordata*

Persea indica (Laurus indica)

Persea macrantha

Persea macrantha

Persea macrantha

Persea palustris
Armatella caulicola

Hosag. et al. 2005

Biju et al.

Meliola litseicola Hansf.

1946

Meliola mannavanensis

Hosag. et al. 2000

Meliola palakkadensis

Hosag. et al. 2007

Meliola pudukadensis

Hosag. 1988

Meliola rajamalaënsis

Hosag. et al. 2005

Meliola sempeiensis $\mathrm{W}$.

Yamam. 1941

Meliola suttonii Hosag. et al. 2005

!Meliola litseae var.

keralensis Hosag. 1990

Meliola machili W.

Yamam. 1941

Meliola dactylopoda Syd.

1928

Asteridiella nectandrae

(Hansf.) Hansf. 1961

Meliola magna F. Stevens

1928

Appendiculella neolitseae

B. Song 1998

Armatella litseae (Henn.)

Theiss. \& Syd. 1915

Armatella yatesii Hansf.

1949

Meliola sempeiensis W.

Yamam. 1941

Armatella phoebeicola

Hosag. 1991

Irenopsis ocoteae (F.

Stevens) F. Stevens 1927

Meliola ocoteicola $\mathrm{F}$.

Stevens 1916

Meliola whetzelii Hansf. Hansford

1957

$1956 \mathrm{~b}$

Asteridiella perseae (F. Hansford

Stevens) Hansf. $1961 \quad 1961$

Asteridiella perseae (F. Hansford

Stevens) Hansf. $1961 \quad 1961$

Meliola drepanochaeta Sydow 1926

Syd. 1926

Meliola maculans

Hansford

1961

Hosagoudar

1987 a

Armatella katumotoi

Hosag. 1988

Hansford

$1956 b$

1957

Hosagoudar

$1987 \mathrm{~b}$

Hosag. 1989

Hansford

Meliola floridensis Hansf. 1957

$1956 b$ 


\begin{tabular}{|c|c|c|c|}
\hline & Persea palustris & $\begin{array}{l}\text { Setameliola martiniana } \\
\text { (Gaillard) D.R. Reynolds }\end{array}$ & $\begin{array}{l}\text { Reynolds } \\
2010\end{array}$ \\
\hline & Persea petiolaris & $\begin{array}{l}\text { Meliola antioquensis } \\
\text { Garcés } 1944\end{array}$ & Orejuela 1944 \\
\hline & Persea sp. & Asteridiella manca (Ellis & Hansford \\
\hline & & \& G. Martin) Hansf. 1961 & 1961 \\
\hline & Phoebe sp. & Meliola cumbrensis & Hansford \\
\hline \multirow{11}{*}{ Lecythidaceae } & Barringtonia acutangula & $\begin{array}{l}\text { Hansf. } 1957 \\
\text { Meliola indica Svd \& P }\end{array}$ & $\begin{array}{l}1956 b \\
\text { Sydow et al. }\end{array}$ \\
\hline & & Syd. 1911 & 1911 \\
\hline & $\begin{array}{l}\text { Barringtonia acutangula } \\
\text { (Barringtonia luzonensis) }\end{array}$ & $\begin{array}{l}\text { Meliola barringtoniae } \\
\text { H.S. Yates } 1917\end{array}$ & Yates 1917 \\
\hline & Barringtonia racemosa & $\begin{array}{l}\text { Meliola racemosae Mibey } \\
1997\end{array}$ & $\begin{array}{l}\text { Mibey \& } \\
\text { Hawksworth } \\
1997\end{array}$ \\
\hline & Barringtonia sp. & $\begin{array}{l}\text { Meliola barringtoniicola } \\
\text { F. Stevens \& Roldan ex } \\
\text { Hansf. } 1963\end{array}$ & $\begin{array}{l}\text { Hansford } \\
1962\end{array}$ \\
\hline & Careya arborea & $\begin{array}{l}\text { Meliola careyae }(\mathrm{F} . \\
\text { Stevens) Hosag. } 2003\end{array}$ & $\begin{array}{l}\text { Hosagoudar } \\
\text { 2003a }\end{array}$ \\
\hline & Eschweilera ovata & $\begin{array}{l}\text { !Irenopsis masakensis } \\
\text { var. major Bat. \& H. } \\
\text { Maia } 1965\end{array}$ & $\begin{array}{l}\text { Hansford } \\
1941\end{array}$ \\
\hline & Gustavia angusta\# & $\begin{array}{l}\text { Irenopsis gustaviae } \\
\text { Hansf } 1957\end{array}$ & $\begin{array}{l}\text { Hansford } \\
1957 b\end{array}$ \\
\hline & Napoleonaea heudelotii & Meliola napoleonae & Hansford \& \\
\hline & & Hansf. \& Deighton 1948 & $\begin{array}{l}\text { Deighton } \\
1948\end{array}$ \\
\hline & Scytopetalum tieghemii* & $\begin{array}{l}\text { Meliola scytopetali Hansf. } \\
\text { \& Deighton } 1948\end{array}$ & $\begin{array}{l}\text { Hansford \& } \\
\text { Deighton } \\
1948\end{array}$ \\
\hline \multirow[t]{2}{*}{ Linaceae } & Hugonia castaneifolia* & $\begin{array}{l}\text { Meliola castaneifoliae } \\
\text { Mibey } 1997\end{array}$ & $\begin{array}{l}\text { Mibey \& } \\
\text { Hawksworth } \\
1997\end{array}$ \\
\hline & Hugonia planchonii & $\begin{array}{l}\text { Meliola hugoniae Hansf. } \\
\text { \& Deighton } 1948\end{array}$ & $\begin{array}{l}\text { Hansford \& } \\
\text { Deighton } \\
1948\end{array}$ \\
\hline Linderniaceae & Torenia travancorica* & $\begin{array}{l}\text { Meliola toreniae Hosag. } \\
1996\end{array}$ & $\begin{array}{l}\text { Hosagoudar } \\
1996\end{array}$ \\
\hline \multirow[t]{8}{*}{ Loganiaceae } & Couthovia novocaledonica* & $\begin{array}{l}\text { Meliola couthoviae B. } \\
\text { Huguenin } 1969\end{array}$ & $\begin{array}{l}\text { Index } \\
\text { Fungorum } \\
2016\end{array}$ \\
\hline & Gardneria ovata & $\begin{array}{l}\text { Meliola gardneriae } \\
\text { Hansf. \& Thirum. } 1948\end{array}$ & $\begin{array}{l}\text { Hansford \& } \\
\text { Thirumalacha } \\
\text { r } 1948\end{array}$ \\
\hline & Gardneria sp. & $\begin{array}{l}\text { Meliola gardneriae } \\
\text { Hansf. \& Thirum. } 1948\end{array}$ & $\begin{array}{l}\text { Hansford \& } \\
\text { Thirumalacha } \\
\text { r } 1948\end{array}$ \\
\hline & $\begin{array}{l}\text { Geniostoma densiflorum var. } \\
\text { oleifolium (Geniostoma } \\
\text { oleifolium) }\end{array}$ & $\begin{array}{l}\text { Amazonia geniostomatia } \\
\text { B. Huguenin } 1969\end{array}$ & $\begin{array}{l}\text { Huguenin } \\
1969\end{array}$ \\
\hline & Labordia sp. & $\begin{array}{l}\text { Amazonia psychotriae } \\
\text { (Henn.) Theiss. } 1913\end{array}$ & Theissen 1913 \\
\hline & Spigelia sp. & $\begin{array}{l}\text { Meliola spigeliae Hansf. } \\
1955\end{array}$ & $\begin{array}{l}\text { Hansford } \\
1955\end{array}$ \\
\hline & & $\begin{array}{l}\text { Meliola andamanica } \\
\text { Hosag. et al. } 2014\end{array}$ & $\begin{array}{l}\text { Hosagoudar et } \\
\text { al. } 2014\end{array}$ \\
\hline & Strychnos colubrina* & $\begin{array}{l}\text { Meliola kodaguensis } \\
\text { Hosag. et al. } 2013\end{array}$ & $\begin{array}{l}\text { Thimmaiah et } \\
\text { al. } 2013\end{array}$ \\
\hline
\end{tabular}


Strychnos multiflora*

Strychnos nux-vomica

Strychnos nux-vomica

Strychnos nux-vomica

Strychnos nux-vomica

Strychnos sp.

Strychnos sp.

Strychnos sp.

Strychnos sp.

Strychnos sp.

Strychnos sp.

Strychnos suberifera*

Usteria guineensis

Loranthaceae

Dendropemon parvifolius

Dendrophthoe falcata

Dendrophthoe falcata

Dendrophthoe fulcata

Englerina parviflora

Helixanthera parasitica

(Loranthus pentapetalus)

Loranthus sp.

Loranthus sp.

Phthirusa pyrifolia

Tapinanthus bangwensis

(Loranthus bangwensis)
Meliola strychnimultiflorae Hansf. 1958

Meliola cannonii Hosag. 2005

Hansford

$1957 \mathrm{c}$

Meliola gamsii Hosag. \&

Shiburaj 2002

Hosagoudar 2005

Hosagoudar

$\&$ Shiburaj

2002

Meliola petchii Hansf. Hansford

1946

1946

Meliola strychni-nux-

Gawande et

vomicae Gawande et al.

2004

Meliola evanida Gaillard

1892

al. 2004

Index

Fungorum

2016

Meliola Mibey \&

longiappressoriata Mibey Cannon 1999

\& P.F. Cannon 1999

Meliola strychnacearum

Hosag. \& T.K. Abraham 1998

Meliola strychni Mibey 1997

Hosagoudar et al. $1998 \mathrm{e}$

Mibey \& Hawksworth 1997

Meliola strychnigena Hosagoudar

Hosag. \& Manojk. 2004 2004b

Meliola warneckei Hansf. Hansford

$1955 \quad 1955$

Meliola strychnicola Gaillard 1892

Gaillard 1892

Meliola usteriae Hansf. \& Hansford \& Deighton 1948

Deighton

1948

Meliola biparasitica Cif. $\quad$ Ciferri 1938

1938

Meliola glaziovii Hansf. Hansford

1957

$1957 b$

Meliola dendropemonis

Petrak \&

Petr. \& Cif. 1930

Ciferri 1930

Meliola loranthacearum

Hosag. \& T.K. Abraham 1998

Hosagoudar

\& Abraham

$1998 b$

Meliola prataprajii Index

Hosag. \& T.K. Abraham Fungorum

1996

2016

Meliola dendrophthoicola Hosagoudar

Hosag. \& T.K. Abraham \& Abraham

1999

1999a

Meliola englerinae

Deighton

Deighton 1958

1957

Irenopsis loranthi Y.X.

$\mathrm{Hu} 1993$

Irenopsis loranthicola

Hosag. \& Riju 2011

Hu et al.

$1993 b$

Hosagoudar

Meliola catubigensis H.S. Yates 1917

Yates 1917

Meliola phthirusae Hansf. Hansford 1957

$1956 b$

Meliola loranthi Gaillard Gaillard 1892 1892 


\begin{tabular}{|c|c|c|c|}
\hline & Taxillus nigrans (Loranthus & Meliola suisyaensis $\mathrm{W}$. & Yamamoto \\
\hline & lonicerlifolius) & Yamam. 1940 & 1940 \\
\hline Lygodiaceae & Lygodium oligostachyum & Irenopsis ekmanii (Cif.) & Hansford \\
\hline \multirow[t]{4}{*}{ Lythraceae } & Duabanga grandiflora & $\begin{array}{l}\text { Meliola duabangae Y.X. } \\
\mathrm{Hu} 1993\end{array}$ & $\begin{array}{l}\text { Hu et al. } \\
1993 b\end{array}$ \\
\hline & Lagerstroemia indica & $\begin{array}{l}\text { Irenopsis lagerstroemiae } \\
\text { Bat. \& Nascim. } 1956\end{array}$ & $\begin{array}{l}\text { Batista et al. } \\
1956\end{array}$ \\
\hline & Lagerstroemia sp. & $\begin{array}{l}\text { Irenopsis lagerstroemiae } \\
\text { Bat. \& Nascim. } 1956\end{array}$ & $\begin{array}{l}\text { Batista et al. } \\
1956\end{array}$ \\
\hline & Woodfordia fruticosa & $\begin{array}{l}\text { Meliola woodfordiae } \\
\text { Srinivas. } 1974\end{array}$ & $\begin{array}{l}\text { Srinivasulu } \\
1974\end{array}$ \\
\hline \multirow[t]{17}{*}{ Magnoliaceae } & Magnolia angatensis & Meliola diplochaeta Syd. & Sydow \& \\
\hline & (Talauma villariana) & \& P. Syd. 1912 & Sydow $1912 b$ \\
\hline & Magnolia champaca & Asteridiella micheliae & Jana et al. \\
\hline & (Michelia champaca) & Jana et al. 2005 & $2005 b$ \\
\hline & $\begin{array}{l}\text { Magnolia champaca } \\
\text { (Michelia champaca) }\end{array}$ & $\begin{array}{l}\text { Asteridiella micheliifolia } \\
\text { Hosag. } 2013\end{array}$ & $\begin{array}{l}\text { Hosagoudar } \\
\text { 2013a }\end{array}$ \\
\hline & $\begin{array}{l}\text { Magnolia champaca } \\
\text { (Michelia champaca) }\end{array}$ & Meliola cariappae Hosag. & Hosagoudar \\
\hline & Magnolia figo (Michelia & Meliola micheliae Hansf. & Hansford \\
\hline & $\begin{array}{l}\text { fuscata) } \\
\text { Magnolia lanuginosa }\end{array}$ & Asteridiella micheliae & $\begin{array}{l}194 / \mathrm{a} \\
\text { Jana et al. }\end{array}$ \\
\hline & (Michelia velutina) & Jana et al. 2005 & $2005 b$ \\
\hline & $\begin{array}{l}\text { Magnolia maudiae (Michelia } \\
\text { maudiae) }\end{array}$ & $\begin{array}{l}\text { Appendiculella } \\
\text { micheliicola J.C. Yang } \\
1989\end{array}$ & Yang 1989 \\
\hline & Magnolia obovata & $\begin{array}{l}\text { Meliola hoehneliana } \\
\text { Hansf. } 1961\end{array}$ & $\begin{array}{l}\text { Hansford } \\
1961\end{array}$ \\
\hline & Magnolia portoricensis & $\begin{array}{l}\text { Meliola magnoliae } \mathrm{F} . \\
\text { Stevens } 1916\end{array}$ & Stevens 1916 \\
\hline & Magnolia virginiana & $\begin{array}{l}\text { Meliola nashii Hansf. } \\
1955\end{array}$ & $\begin{array}{l}\text { Hansford } \\
1953 b\end{array}$ \\
\hline & Mikania cordifolia & $\begin{array}{l}\text { Appendiculella } \\
\text { compositarum (Earle) } \\
\text { Toro } 1925\end{array}$ & Toro 1925 \\
\hline & Mikania micrantha & $\begin{array}{l}\text { Appendiculella sororcula } \\
\text { (Speg.) Hansf. } 1961\end{array}$ & $\begin{array}{l}\text { Hansford } \\
1961\end{array}$ \\
\hline & Mikania scandens & $\begin{array}{l}\text { Appendiculella sororcula } \\
\text { (Speg.) Hansf. } 1961\end{array}$ & $\begin{array}{l}\text { Hansford } \\
1961\end{array}$ \\
\hline & Talauma sp. & $\begin{array}{l}\text { Meliola talaumae Hansf. } \\
1954\end{array}$ & $\begin{array}{l}\text { Hansford } \\
1954\end{array}$ \\
\hline \multirow[t]{9}{*}{ Malpighiaceae } & - & $\begin{array}{l}\text { Irenopsis malpighiicola } \\
\text { Hansf. } 1955\end{array}$ & $\begin{array}{l}\text { Hansford } \\
1955\end{array}$ \\
\hline & - & Meliola crenato-furcata & Sydow \& \\
\hline & - & Syd. \& P. Syd. 1916 & $\begin{array}{l}\text { Sydow } 1916 a \\
\text { Hansford }\end{array}$ \\
\hline & & Hansf. 1955 & 1955 \\
\hline & Acridocarpus natalitius & $\begin{array}{l}\text { Meliola acridocarpi } \\
\text { Doidge } 1941\end{array}$ & Doidge 1941 \\
\hline & Acridocarpus natalitius & $\begin{array}{l}\text { Meliola bifida Cooke } \\
1880\end{array}$ & Cooke 1880 \\
\hline & Acridocarpus plagiopterus & $\begin{array}{l}\text { Irenopsis acridocarpicola } \\
\text { Hansf. \& Deighton } 1948\end{array}$ & $\begin{array}{l}\text { Hansford \& } \\
\text { Deighton } \\
1948\end{array}$ \\
\hline & Acridocarpus sp. & $\begin{array}{l}\text { Irenopsis stuhlmanniana } \\
\text { (Henn.) Hansf. } 1957\end{array}$ & $\begin{array}{l}\text { Hansford } \\
1956 b\end{array}$ \\
\hline & Banisteria sp.* & $\begin{array}{l}\text { Irenopsis rectangularis } \\
\text { (F. Stevens) F. Stevens } \\
1927\end{array}$ & Stevens 1927 \\
\hline
\end{tabular}


Bunchosia glandulosa

Bunchosia nitida (Bunchosia cornifolia)

Byrsonima crassifolia

Byrsonima lucida

Byrsonima sericea

Byrsonima sp.

Byrsonima sp.

Byrsonima sp.

Heteropterys laurifolia

(Banisteria laurifolia)

Hiptage bengalensis*

Hiptage sp.

Malpighia glabra

Malpighia glabra

Stigmaphyllon angulosum

Stigmaphyllon convolvulifolium

Stigmaphyllon ellipticum

Stigmaphyllon emarginatum (Stigmaphyllon lingulatum)

Stigmaphyllon emarginatum (Stigmaphyllon lingulatum)

Triaspis stipulata

Triopterys jamaicensis*

Malvaceae

Abutilon ramosum

Abutilon sp.

Bastardiopsis sp.

Byttneria ramosissima

Byttneria sp.

Christiana africana

Cola lateritia

Cola nitida
Asteridiella cuspidata

(Cif.) Hansf. 1961

Meliola crenata $\mathrm{G}$.

Winter 1892

Hansford

1961

Index

Fungorum

2016

Meliola byrsonimae F. Stevens 1916

Stevens 1916

Meliola byrsonimae F. Stevens 1916

Stevens 1916

Meliola byrsonimae F. Stevens 1916

Stevens 1916

Meliola byrsonimicola $\mathrm{F}$. Stevens \&

Stevens \& Tehon 1926 Tehon 1926

Meliola byrsonimina F. Stevens \&

Stevens \& Tehon 1926 Tehon 1926

Meliola cibaoensis Hansf. Hansford 1955

1955

Irenopsis banisteriae

Hansf. 1957

Hansford

$1956 b$

Irenopsis hiptages $\mathrm{W}$. Index

Yamam. 1941

Fungorum

2016

!Irenopsis hiptages var. Hosagoudar indica Hosag. \& Sabeena 2013a 2013

Irenopsis malpighiae Hansford

Hansf. 1949

Meliola xenoderma Syd. Sydow 1926

1926

Asteridiella ozamensis Hansford

(Cif.) Hansf. $1961 \quad 1961$

Irenopsis circinata Hansf. Hansford

$1957 \quad 1956 b$

Asteridiella stigmaphylli Hansford

(Petr.) Hansf. 1961

Asteridiella stellata (Cif.) Hansford

Hansf. 1961

Irenopsis chaethachna Hansford

(Cif.) Hansf. 1961

Irenopsis triaspidis Deighton

Deighton 1958

Meliola danertii Schmied. Schmiedeknec

1989

Amazonia abutilonis

Hosag. 1991

ht 1989

Hosagoudar

1991a

Irenopsis sidae (Rehm) S. Hughes 1952

Hughes 1952

Irenopsis bastardiopsidis Stevens 1927

(Speg.) F. Stevens 1927

Asteridiella buettneriae

(J.A. Stev.) Hansf. 1961

Irenopsis buettneriicola

Deighton 1951

Irenopsis coronata

(Speg.) F. Stevens 1927

Irenopsis media Deighton

1958

Hansford

1961

Deighton

1951

Stevens 1927

Deighton

1957

Irenopsis coliicola Hansf. Hansford \&

\& Deighton 1948
Deighton

1948 
Cola nitida

Cola simiarum

Cola sp.

Cola sp.

Cola sp.

Cola verticillata

Columbia serratifolia*

Dombeya buettneri

Durio zibethinus

Eriolaena quinquelocularis

Eriolaena sp.

Firmiana simplex (Sterculia urens)

Glyphaea brevis (Glyphaea grewioides)

Glyphaea brevis (Glyphaea

lateriflora)

Grewia asiatica (Grewia

subinaequalis)

Grewia sp.

Grewia sp.

Grewia tiliifolia

Guazuma ulmifolia

Helicteres isora

Hibiscus esculentus

Hibiscus mutabilis

Hibiscus sp.

Hibiscus tilliaceus

Hibiscus tilliaceus

Hibiscus tilliaceus

Hoheria sexstylosa
Irenopsis njalaensis

Hansf. \& Deighton 1948

Meliola colae-simiarum

Deighton 1958

Amazonia colae Mibey

1997

Irenopsis colae Hansf.

1946

Irenopsis keniensis Mibey

1997

Irenopsis aburiensis

Deighton 1958

Irenopsis coronata

(Speg.) F. Stevens 1927

Appendiculella dombeyae

(Hansf. \& Deighton)

Hansf. 1961

Meliola durionis Hansf.

1957

Irenopsis eriolaenae

Hosag. 1989

Meliola eriolaenicola B.

Song \& Y.H. Shen 2002

Asteridiella anamalaiana

Hosag. 1991

Asteridiella glyphaeae

(Hansf.) Hansf. 1961

Irenopsis coronata

(Speg.) F. Stevens 1927

Asteridiella grewiae C.R.

Patil ex Hosag. 1996

Irenopsis coimbatorica

Hosag. et al. 1996

Meliola grewiicola Hansf.

1958

\#Meliola grewiae

Srinivas. 1974

Meliola monilipes Cif. 1954

Irenopsis helicteris

Hosag. 1991

Meliola hibisci G. Arnaud 1918

Meliola mutabilidis

Hosag. et al. 2013

Irenopsis leeae Hansf.

1937

Irenopsis coronata

(Speg.) F. Stevens 1927

Irenopsis moelleriana (G. Stevens 1927

Winter) F. Stevens 1927

Meliola procera Cif. 1938

Irenopsis hoheriae Hansf. 1955
Hansford \&

Deighton

1948

Deighton

1957

Mibey \&

Hawksworth

1997

Hansford

1946

Mibey \&

Hawksworth

1997

Deighton

1957

Stevens 1927

Hansford

1961

Hansford

$1956 \mathrm{~b}$

Hosagoudar

\& Goos 1989

Song et al.

2002d

Hosagoudar

\& Goos 1991

Hansford

1961

Stevens 1927

Hosagoudar

1996

Hosagoudar 1996

Hansford

1957c

Srinivasulu

1974

Ciferri 1954

Hosagoudar

1991a

Farr \&

Rossman

2016

Hosagoudar

2013a

Hansford

1937

Stevens 1927

Ciferri 1938

Hansford

1955 
Julostylis polyandra*

Kleinhovia hospita

Kydia calycina

Kydia calycina

Leptonychia pubescens

Luehea divaricata

Luehea grandiflora

Microcos paniculata*

Microcos triflora (Grewia stylocarpa)

Nesogordonia papaverifera

Pachira emarginata

Pavonia paludicola (Pavonia spicata)

Pavonia sp.

Pterospermum javanicum*

Pterospermum obliquum*

Pterospermum reticulatum*

Pterospermum sp.

Pterygota alata

Pterygota alata

Reevesia pubescens

Sida cordata

Sida javensis

Sida sp.

Sida sp.

Sterculia lanceifolia

(Sterculia roxburghii)
Asteridiella julostylidis Hosag. \& T.K. Abraham 1998

Irenopsis kleinhoviae Hosag. \& Archana 2010

Irenopsis mudumalaiensis Hosag. 1991

Meliola kydiae-calycinae Hansf. \& Thirum. 1948

Irenopsis leptonychiae Deighton 1958

Irenopsis coronata (Speg.) F. Stevens 1927

Irenopsis luheaegrandiflorae D.B. Pinho \& O.L. Pereira 2012 Meliola thirumalacharii Hosag. \& Rajendran 1989

Meliola grewiae Hansf. 1957

Irenopsis nesogordoniae Deighton 1958

Asteridiella tonsa (Cif.) Hansf. 1961

Asteridiella pavoniae (Cif.) Hansf. 1961

Irenopsis pavoniae Hosag. \& Jac. Thomas 2010 Irenopsis tjibodensis Hansf. 1954 Meliola pterospermicola F. Stevens \& Roldan ex Hansf. 1963

Meliola pterospermi $\mathrm{F}$. Stevens 1928

Meliola pterospermi F. Stevens 1928

Stevens 1928

Irenopsis pterygotae Hosag 2011

Meliola pterygotae

Hosag. et al. 2006

Meliola reevesiae Maity 1978 2016

Hosagoudar $\&$ Sabeena 2011a Hosagoudar et al. $2006 \mathrm{~b}$

Index

Fungorum

Irenopsis sidae (Rehm) S. Hughes 1952 Hughes 1952

Irenopsis sidae (Rehm) S. Hughes 1952 Hughes 1952

Irenopsis sidicola $(\mathrm{F} . \quad$ Hansford Stevens \& Tehon) Hansf. 1961

1961

Meliola goianensis Bat. \& Batista et al. H. Maia 1965

1965a

Irenopsis sterculiae Jana Jana et al. et al. 2006 2006 
Sterculia sp.

Sterculia sp.

Sterculia tragacantha

Tarrietia utilis

Theobroma cacao

Theobroma cacao

Thespesia lampas

Triplochiton scleroxylon

Triumfetta annua

Triumfetta semitriloba

Triumfetta semitriloba

Triumfetta sp.

Triumfetta sp.

Marantaceae

Marattiaceae

Angiopteris evecta

Marcgraviaceae
Calathea crotalifera

(Calathea insignis)

Calathea crotalifera

(Calathea insignis)

Calathea lutea

Calathea macrosepala

Calathea sp.

Calathea tuberosa

Donax canniformis

Hypselodelphys ?poggeana

Indianthus virgatus

(Schumannianthus virgatus)

Maranta arundinacea

Marantochloa sp.

Marcgravia flagellaris

Marcgravia nepenthoides
Meliola sterculiacearum

Hosag. \& Kamar. 2005

Meliola sterculiicola

Hosag. \& P.J. Robin 2011

Meliola sterculiae Hansf.

\& Deighton 1948

Irenopsis tarrietiae

Hansf. \& Deighton 1948

Irenopsis guianensis (F.

Stevens \& R.I. Dowell) F.

Stevens 1927

Meliola theobromae

Faber 1909

Irenopsis thespesiae

Hansf. 1954

Meliola triplochitonis S. Hughes 1953

Hughes 1953

Meliola yunnanensis G.Z. Jiang 1986a

Jiang 1987

Irenopsis coronata

(Speg.) F. Stevens 1927

Irenopsis lagunae Hansf. Hansford

1946

1946

Asteridiella arachnoidea

(Speg.) Hansf. 1961

Hansford

1961

Irenopsis aciculosa $(\mathrm{G}$.

Winter) F. Stevens 1927

Meliola calatheae $\mathrm{F}$.

Stevens 1928

Meliola marantacearum

F. Stevens 1928

Meliola calatheicola $\mathrm{F}$. Stevens 1928

Stevens 1928

Meliola hispida $\mathrm{F}$.

Stevens 1928

Meliola telensis Hansf. Hansford

1957

$1956 \mathrm{~b}$

Meliola calatheicola F. Stevens 1928

Stevens 1928

Meliola heterotricha Syd. \& P. Syd. 1913

Meliola hypselodelphidis

S. Hughes 1952

Asteridiella

schumannianthi Hosag. et al. 1999

Meliola marantae F.

Stevens 1928

Meliola marantochloae

Hansf. 1946

Meliola angiopteridis

Hansf. 1954

Asteridiella marcgraviae

Henn. 1904

Irenopsis ramonensis

(Syd.) F. Stevens 1927
Sydow \&

Sydow 1913b

Hughes 1952

Hosagoudar et al. 1999 b

Stevens 1928

Hansford

1946

Hansford

1954

Hennings

$1904 b$

Stevens 1927 
Marcgravia rectiflora

Marcgravia rectiflora

Marcgravia sp.

Melastomataceae

Acinodendron sintenisii

(Miconia sintenisii)

Brachyotum campanulare

(Arthrostemma campanulare)

Clidemia hirta

Conostegia xalapensis

Conostegia xalapensis

(Conostegia lanceolata)

Dissochaeta gracilis*

Dissotis paucistellata*

Henriettella fascicularis

Lijndenia fragrans

(Memecylon fragrans)

Medinilla disparifolia*

Memecylon depressum

Memecylon edule*

Memecylon edule*

Memecylon fasciculare*

Memecylon gracile*

Memecylon lanceolatum

Memecylon lanceolatum

Memecylon sp.

Memecylon sp.

Memecylon urdanetense*
\#Meliola marcgraviae

Tehon 1919

Asteridiella cyphopoda

(Cif.) Hansf. 1961

Asteridiella

marcgraviicola Hansf.

1958

Asteridiella arachnoidea

(Speg.) Hansf. 1961

Meliola inconspicua

Hansf. 1957

Irenopsis miconiicola $(\mathrm{F}$.

Stevens) F. Stevens 1927

Asteridiella

melastomatacearum

(Speg.) Hansf. 1961

Asteridiella

melastomatacearum

(Speg.) Hansf. 1961

Irenopsis conostegiae $\mathrm{F}$.

Stevens 1927

Meliola brachycera Syd. Sydow 1926

1926

Meliola dissotidis Hansf. Hansford \&

\& Deighton 1948

Deighton

1948

Meliola dissotidis Hansf.

\& Deighton 1948

Hansford \&

Deighton

1948

Irenopsis miconiicola $(\mathrm{F}$.

Stevens) F. Stevens 1927

Meliola fragrans Mibey

\& P.F. Cannon 1999

Stevens 1927

Mibey \&

Cannon 1999

Meliola medinillae Hansf. Hansford

1955

1955

Meliola memecylicola

Hansf. 1957

Hansford

$1956 b$

Meliola affinis Syd. \& P.

Syd. 1913

Meliola memecylicola

Hansf. 1957

Meliola disciseta Roger

1936

Sydow \&

Sydow 1913b

Hansford

$1956 b$

Index

Fungorum

2016

Meliola memecyli Syd. \& P. Syd. 1917

Meliola memecyli Syd. \& P. Syd. 1917

Meliola memecylicola

Hansf. 1957

Sydow \&

Sydow 1917a

Sydow \&

Sydow 1917a

Hansford

$1956 \mathrm{~b}$

Meliola attayarica Hosag.

\& T.K. Abraham 1999

Meliola hendeloti Gaillard

1892

Hosagoudar

\& Abraham

1999b

Index

Fungorum

2016

Meliola affinis Syd. \& P. Sydow \&

Syd. 1913 
Miconia argentea

Miconia argentea

Miconia laevigata

Miconia prasina

Miconia prasina

Mouriri domingensis

Mouriri sp.

Meliaceae

Aglaia edulis

Aglaia palembanica

Aglaia sp.

Aglaia sp.

Aglaia sp.

Aglaia sp.

Aglaia sp.

Aglaia tomentosa (Aglaia minutiflora)

Amoora rohituka*

Amoora sp.*

Aphanamixis polystachya

Aphanamixis polystachya
\#Irenina shropshiriana F. Stevens 1927

Stevens 1927

Irenopsis shropshiriana Hansford

(F. Stevens) Hansf. 19611961

Asteridiella

melastomatacearum

(Speg.) Hansf. 1961

Asteridiella

melastomatacearum

(Speg.) Hansf. 1961

Irenopsis miconiae $(\mathrm{F}$.

Hansford

1961

Hansford

1961

Stevens) F. Stevens 1927

Meliola mouririae Cif.

1931

Meliola camaragibeicola Batista et al.

Bat. \& H. Maia 1956

Asteridiella obesa (Speg.) Hansford

Hansf. 1961

Asteridiella

1961

Hansford

pseudekebergiae (Hansf.) 1961

Hansf. 1961

Meliola arkevermae

Hosag. \& Sabeena 2012

Hosagoudar

$\&$ Sabeena

2012

Meliola parvula Syd. \& P. Sydow \&

Syd. 1913

Sydow 1913b

Meliola rickii Hansf. 1955 Hansford

$1953 b$

Meliola silentvalleyensis Hosagoudar

Hosag. 2006

Meliola aglaiae-edulis

\& Biju 2006

Jana et al.

2005 c

Jana et al. 2005

Sydow 1923b

P. Syd. 1923

Meliola aglaiae Syd. \& P. Sydow \&

Syd. 1914

Sydow $1914 \mathrm{c}$

Meliola aglaiicola Hansf. Hansford

1954

1954

Meliola aglaina Hansf.

1955

Hansford

1955

Meliola savarkarii Hosag. Hosagoudar et et al. 2014

al. 2014

Meliola vazhachalensis

Hosag. \& Jac. Thomas

2009

Hosagoudar

\& Thomas

2009

Meliola sairandhriana

Hosag. \& Archana 2009

Asteridiella indica

Hosagoudar

\& Archana

2009

Index

(Anahosur) A. Pande

2008

Fungorum

2016

Meliola amoorae H.S. $\quad$ Yates 1918

Yates 1918

Meliola aphanamixidis

Hosag. 1990

Hosagoudar

\& Goos

$1990 \mathrm{~b}$

Meliola nairii Hosag.

1990
Hosagoudar

\& Goos

1990b 
Carapa guianensis

Carapa procera

Chukrasia sp

Chukrasia sp.

Chukrasia tabularis

Cipadessa baccifera

Dysoxylum arborescens

Dysoxylum cumingianum

Dysoxylum malabaricum

Dysoxylum rufescens

(Dysoxylum nitidum)

Dysoxylum sp.

Dysoxylum sp.

Dysoxylum sp.

Dysoxylum sp.

Dysoxylum sp.

Dysoxylum sp.

Dysoxylum sp.

Dysoxylum sp.

Ekebergia pterophylla

Ekebergia sp.

Entandrophragma angolense

Entandrophragma angolense

Entandrophragma sp.

Guarea guidonia

Guarea guidonia (Guarea trichilioides)

Guarea kunthiana
Meliola trifurcata Cif. 1938

Meliola carapae Hansf. \& Hansford \& Deighton 1948

Deighton

1948

Meliola chukrasiicola

Hosag. et al. 2014

Meliola chukrasiae

Hosag. 1996

Irenopsis chukrasiae

Hosag. 1994

Meliola cipadessae

Hosag. et al. 2010

Hosagoudar et

al. 2014

Hosagoudar

1996

Hosagoudar et al. 1994 b

Hosagoudar et al. 2010c

Irenopsis dysoxyli Jana et Jana et al. al. 2005

$2005 b$

Meliola meliacearum F. Hansford

Stevens \& Roldan ex 1962

Hansf. 1963

Meliola dysoxyli-

malabarici Hosag. \&

Kamar. 2002

Meliola dysoxyli-nitidi B. Index

Huguenin 1969

Fungorum

2016

Meliola banahaensis H.S. Yates 1918

Yates 1918

Meliola dysoxyli Hansf. Hansford

1953

1953a

Meliola dysoxylicola

Hansf. 1953

Hansford

1953a

Meliola dysoxyligena

Hosag. \& Riju 2011

Hosagoudar

\& Riju 2011a

Meliola dysoxylina Hansf. Hansford

1961

1961

Meliola macalpinei Sacc.

\& P. Syd. 1899

Saccardo \&

Meliola trifurcata Cif.

1938

Meliola zamboagensis

Hansf. 1957

Asteridiella ekebergiae

(Doidge) Hansf. 1961

Meliola ekebergiae

Hansf. 1937

Meliola bunyorensis

Hansf. 1938

Sydow 1899

Ciferri 1938

Hansford

$1957 \mathrm{~b}$

Hansford

1961

Hansford

1937

Hansford

1938

Meliola turraeae Hansf. Hansford 1944

1944

Hansford

1961

entandrophragmatis

(Hansf.) Hansf. 1961

Meliola vicosensis D.B. Pinho et al.

Pinho \& O.L. Pereira

2013

Meliola guareicola $\mathrm{F}$.

Stevens 1916

Meliola vicosensis D.B. Pinho et al.

Pinho \& O.L. Pereira

2013
2013

Stevens 1916

2013 
Guarea macrophylla subsp. Spiciflora (Guarea balansae)

Guarea sp.

Guarea sp.

Guarea sp.

Guarea sp.

Heynea trijuga

Khaya anthotheca

Khaya grandifoliola

Lovoa brownii*

Reinwardtiodendron anamalaiense

Reinwardtiodendron sp.

Sandoricum koetjape (Sandoricum indicum) Sandoricum sp.

Sapindus saponaria

Swietenia mahagoni

Swietenia mahagoni

Toona ciliata

Trichilia catigua

Trichilia claussenii (Trichilia triphyllaria)

Trichilia emetica

Trichilia emetica

Trichilia heudelotii

Trichilia heudelotii

Trichilia pallida

Trichilia prieuriana

Trichilia prieuriana

Trichilia retusa

Trichilia retusa
Meliola guareae Speg.

1912

Spegazzini

Asteridiella parasitica (F. Hansford Stevens) Hansf. $1961 \quad 1961$

Meliola guareella Hansf. Hansford 1957

$1957 b$

Meliola guareina Hansf. Hansford 1957

$1956 \mathrm{~b}$

Meliola platysperma

Theissen 1914

Theiss. 1914

Meliola heyneae Hansf. \& Hansford \& Thirum. 1948

Thirumalacha r 1948

Meliola khayae Hansf. Hansford 1938

1938

Meliola khayae Hansf. 1938

Asteridiella lovoae

(Hansf.) Hansf. 1961

Meliola

reinwardtiodendri Hosag. 1996

Meliola

reinwartiodendricola

Hosag. \& B. Divya 2009

Asteridiella sandorici

(Rehm) Hansf. 1961

Asteridiella sandorici

(Rehm) Hansf. 1961

Asteridiella bomplandi

(Speg.) Hansf. 1961

Hansford

1938

Hansford

1961

Hosagoudar

1996

Hosagoudar

\& Divya 2013

Hansford

1961

Hansford

1961

Hansford

1961

Meliola swieteniae Cif.

1933

Meliola swieteniicola

Hosag. et al. 1994

Ciferri 1933

Meliola toonae Hosag. \&

T. Sabu 2001

Meliola trichiliicola Speg. 1924

Meliola atrovelutina

Speg. 1924

Meliola sinuosa Doidge 1917

Meliola trichiliae Beeli

1920

Asteridiella bersamae

(Hansf.) Hansf. 1961

Meliola togoensis S.

Hughes 1953

Meliola vicosensis D.B. Pinho \& O.L. Pereira 2013

Meliola opposita Syd. \& P. Syd. 1913

Meliola togoensis S.

Hughes 1953

Asteridiella bersamae

(Hansf.) Hansf. 1961

Meliola trichiliae Beeli 1920
Hosagoudar et al. 1994 a

Hosagoudar et al. 2001b

Spegazzini

1924

Spegazzini

1924

Doidge 1917

Beeli 1920

Hansford

1961

Hughes 1953

Pinho et al.

2013

Sydow \&

Sydow 1913b

Hughes 1953

Hansford

1961

Beeli 1920 


\begin{tabular}{|c|c|c|c|}
\hline & Trichilia sp. & Irenopsis trichiliae & Hosagoudar \\
\hline & & Hosag. \& Riju 2010 & \& Riju 2010 \\
\hline & Trichilia sp. & $\begin{array}{l}\text { Meliola thomasii Hansf. } \\
1937\end{array}$ & $\begin{array}{l}\text { Hansford } \\
1937\end{array}$ \\
\hline & Turraea floribunda & $\begin{array}{l}\text { Meliola turraeae Hansf. } \\
1944\end{array}$ & $\begin{array}{l}\text { Hansford } \\
1944\end{array}$ \\
\hline & Vavaea sp. & $\begin{array}{l}\text { Meliola leptochaeta Syd. } \\
\text { \& P. Syd. } 1917\end{array}$ & $\begin{array}{l}\text { Sydow \& } \\
\text { Sydow 1917a }\end{array}$ \\
\hline & Walsura sp. & $\begin{array}{l}\text { Meliola walsurae Hansf. } \\
1958\end{array}$ & $\begin{array}{l}\text { Hansford } \\
1957 \mathrm{c}\end{array}$ \\
\hline & Walsura trifoliolata* & Meliola walsuricola & Biju \& \\
\hline & & Bagool \& H. Biju 2004 & Bagool 2004 \\
\hline & Walsura tubulata & $\begin{array}{l}\text { Irenopsis walsurae X.Y. } \\
\text { Zeng \& K.D. Hyde } 2015\end{array}$ & $\begin{array}{l}\text { Hongsanan et } \\
\text { al. } 2015\end{array}$ \\
\hline & Xylocarpus granatum & Meliola elodea Syd. 1928 & Sydow 1928 \\
\hline Melianthaceae & Bersama sp. & $\begin{array}{l}\text { Asteridiella bersamae } \\
\text { (Hansf.) Hansf. } 1961\end{array}$ & $\begin{array}{l}\text { Hansford } \\
1961\end{array}$ \\
\hline & Bersama sp. & $\begin{array}{l}\text { Asteridiella masakensis } \\
\text { (Hansf.) Hansf. } 1961\end{array}$ & $\begin{array}{l}\text { Hansford } \\
1961\end{array}$ \\
\hline & Bersama sp. & $\begin{array}{l}\text { Meliola bersamae Hansf. } \\
1937\end{array}$ & $\begin{array}{l}\text { Hansford } \\
1937\end{array}$ \\
\hline & Bersama sp. & $\begin{array}{l}\text { Meliola chorleyi Hansf. } \\
1938\end{array}$ & $\begin{array}{l}\text { Hansford } \\
1938\end{array}$ \\
\hline Menispermaceae & - & $\begin{array}{l}\text { Meliola banguiensis H.S. } \\
\text { Yates } 1918\end{array}$ & Yates 1918 \\
\hline & Cissampelos mисronata & $\begin{array}{l}\text { Meliola cissampeli Hansf. } \\
\text { \& F. Stevens } 1937\end{array}$ & $\begin{array}{l}\text { Hansford } \\
1937\end{array}$ \\
\hline & Cissampelos pareira & $\begin{array}{l}\text { Meliola kreiseliana } \\
\text { Schmied. } 1989\end{array}$ & $\begin{array}{l}\text { Schmiedeknec } \\
\text { ht } 1989\end{array}$ \\
\hline & Cissampelos pareira & $\begin{array}{l}\text { Meliola parreirae Bat. } \\
1960\end{array}$ & $\begin{array}{l}\text { Batista et al. } \\
1960\end{array}$ \\
\hline & Cissampelos pareira & Meliola cissampelicola & Hansford \& \\
\hline & (Cissampelos convolvulacea) & Hansf. \& Thirum. 1948 & $\begin{array}{l}\text { Thirumalacha } \\
\text { r } 1948\end{array}$ \\
\hline & Cyclea peltata & $\begin{array}{l}\text { Meliola cycleae Hosag. } \\
1990\end{array}$ & $\begin{array}{l}\text { Hosagoudar } \\
\& \text { Goos 1990a }\end{array}$ \\
\hline & Cyclea peltata & $\begin{array}{l}\text { Meliola subramanyaensis } \\
\text { Hosag. } 2005\end{array}$ & $\begin{array}{l}\text { Hosagoudar et } \\
\text { al. } 2005\end{array}$ \\
\hline & Pericampylus glaucus & Meliola pericampyli $\mathrm{W}$. & Yamamoto \\
\hline & (Pericampylus formosanus) & Yamam. 1941 & $1941 b$ \\
\hline & Stephania hernandiifolia & $\begin{array}{l}\text { Meliola stephaniae Hansf. } \\
1954\end{array}$ & $\begin{array}{l}\text { Hansford } \\
1954\end{array}$ \\
\hline & Tiliacora kenyensis & Meliola phaeomaculans & Mibey \& \\
\hline & & $\begin{array}{l}\text { Mibey \& P.F. Cannon } \\
1999\end{array}$ & Cannon 1999 \\
\hline & Tiliacora sp. & $\begin{array}{l}\text { Meliola cissampeli Hansf. } \\
\text { \& F. Stevens } 1937\end{array}$ & $\begin{array}{l}\text { Hansford } \\
1937\end{array}$ \\
\hline & Tinomiscium petiolare & Meliola tinomisciicola & Hu et al. \\
\hline & (Tinomiscium tonkinense) & Y.X. Hu \& Ouyang 1993 & $1993 b$ \\
\hline & Triclisia subcordata & Meliola pericampyli $\mathrm{W}$. & Yamamoto \\
\hline & & Yamam. 1941 & $1941 b$ \\
\hline Monimiaceae & Hedycarya sp. & $\begin{array}{l}\text { Asteridiella hedycaryae } \\
\text { (Hansf.) Hansf. } 1961\end{array}$ & $\begin{array}{l}\text { Hansford } \\
1961\end{array}$ \\
\hline & Kibara moluccana* & $\begin{array}{l}\text { Meliola megalochaeta } \\
\text { Syd. } 1922\end{array}$ & Sydow $1922 b$ \\
\hline & Mollinedia sp. & $\begin{array}{l}\text { Meliola mollinediae } \\
\text { Theiss. } 1914\end{array}$ & Theissen 1914 \\
\hline & Xymalos monospora & $\begin{array}{l}\text { Meliola rigida Doidge } \\
1917\end{array}$ & Doidge 1917 \\
\hline
\end{tabular}


Antiaris sp.

Antiaris toxicaria

Antiaris toxicaria var. africana (Antiaris africana) Artocarpus glaucus

Artocarpus gomezianus

Artocarpus heterophyllus

Artocarpus sp.

Dorstenia indica

Ficus asperifolia (Ficus urceolaris)

Ficus benjamina

Ficus exasperata

Ficus globosa

Ficus grossularioides (Ficus alba)

Ficus heteropleura (Ficus urophylla)

Ficus lepicarpa

Ficus luschnathiana (Ficus ibapophy)

Ficus maxima (Ficus

rubricosta)

Ficus microcarpa

Ficus nota

Ficus obscura

Ficus odorata

Ficus sarmentosa var. henryi (Ficus foveolata var.

arisanensis)

Ficus sinuata (Ficus rostrata)

Ficus sp.

Ficus sp.

Ficus sp.
Appendiculella echinata (Gaillard) Hansf. 1955

Asteridiella ugandensis

(Hansf.) Hansf. 1961

Asteridiella ugandensis

(Hansf.) Hansf. 1961

Setameliola brinkii

(Hansf.) D.R. Reynolds

2010

Meliola artocarpi H.S.

Yates 1917

Meliola integrifolii C.R.

Patil ex Hosag. 1996

Setameliola artocarpicola

(F. Stevens ex Hansf.)

D.R. Reynolds 2010

Meliola dorsteniae

Hosag. \& T.K. Abraham 1998

Asteridiella ugandensis

(Hansf.) Hansf. 1961

Meliola pulcherrima Syd.

\& P. Syd. 1913

Asteridiella ugandensis

(Hansf.) Hansf. 1961

Meliola fici-globosae

Hansf. 1954

Setameliola microtricha

(Syd. \& P. Syd.) D.R.

Reynolds 2010

Asteridiella umirayensis

(H.S. Yates) Hansf. 1961

Asteridiella umirayensis

(H.S. Yates) Hansf. 1961

Meliola eriophora Speg. 1888

Meliola soroceae Speg.

1912

Asteridiella ficicola

Hosag. et al. 2009

Asteridiella umirayensis

(H.S. Yates) Hansf. 1961

Asteridiella umirayensis

(H.S. Yates) Hansf. 1961

Asteridiella umirayensis

(H.S. Yates) Hansf. 1961

Setameliola sakahensis

(W. Yamam.) D.R.

Reynolds 2010

Asteridiella reticulata (P. Hansford

Karst. \& Roum.) Hansf. 1961

1961

Appendiculella

tonkinensis (P. Karst. \&

Roum.) Toro 1927

Asteridiella cheoi

(Hansf.) Hansf. 1961

Asteridiella fici (Hansf.)

Hansf. 1961
Hansford

1955

Hansford

1961

Hansford

1961

Reynolds

2010

Yates 1917

Hosagoudar 1996

Reynolds

2010

Hosagoudar et

al. 1998d

Hansford

1961

Sydow \&

Sydow 1913c

Hansford

1961

Hansford

1954

Reynolds

2010

Hansford

1961

Hansford

1961

Spegazzini

1888b

Spegazzini

1912

Hosagoudar et

al. 2009

Hansford

1961

Hansford

1961

Hansford

1961

Reynolds

2010

Toro 1927

Hansford

1961

Hansford

1961 
Ficus sp.

Ficus sp.

Ficus sp.

Ficus sp.

Ficus sp.

Ficus sp.

Ficus tinctoria subsp. gibbosa (Ficus gibbosa)

Ficus variegata

Milicia excelsa (Chlorophora excelsa)

Morus alba

Paratrophis glabra*

Pseudomorus pendulina var. australiana*

Sorocea hilarii (Sorocea ilicifolia)

Sorocea hilarii (Sorocea ilicifolia)

Streblus asper

Streblus taxoides

Trilepisium madagascariense

Trilepisium madagascariense

Trilepisium madagascariense (Bosqueia phoberos)

Trophis caucana (Olmedia aspera)

Myricaceae
Meliola bangalorensis Hansf. \& Thirum. 1948

Meliola ficicola Hansf. \& Thirum. 1948

Meliola ficigena Hosag. \& A. Sabeena 2013

Meliola ficium H.S. Yates 1918

Meliola ovatipoda Hansf. \& Thirum. 1948

Meliola zangii B. Song 2003

Meliola kannurensis

Hosag. et al. 2011

Irenopsis benguetensis $\mathrm{F}$.

Stevens \& Roldan ex

Hansf. 1963

Meliola chlorophorae

Hansf. 1946

Meliola mori (Catt.) Sacc.

1882

Setameliola paratrophidis

(Hansf.) D.R. Reynolds

2010

Setameliola pseudomori (Hansf.) D.R. Reynolds 2010

Meliola soroceae Speg.

1912

Meliola soroceana Bat.

1953

Asteridiella strebli Hosag. Hosagoudar et al.. 2004

Meliola strebli Hosag. \& Archana 2009

Cryptomeliola natans

Mibey \& P.F. Cannon 1999

Meliola trilepisii Mibey

\& P.F. Cannon 1999

Meliola soroceae Speg.

1912

Asteridiella olmediae

Hansf. 1958

Asteridiella manca (Ellis

\& G. Martin) Hansf. 1961

Meliola myricae Hansf.

1946

Asteridiella atricha

(Speg.) Hansf. 1961

Asteridiella atricha

(Speg.) Hansf. 1961

Asteridiella manca (Ellis

\& G. Martin) Hansf. 1961
Hansford \&

Thirumalacha r 1948

Hansford \& Thirumalacha r 1948

Hosagoudar \& Sabeena 2013b

Yates 1918

Hansford \&

Thirumalacha r 1948

Song \& Li 2003

Hosagoudar et al. 2011a

Hansford

1962

Hansford

1946

Saccardo

1882

Reynolds

2010

Reynolds

2010

Spegazzini

1912

Index

Fungorum

2016

2004a

Hosagoudar

\& Archana

2009

Mibey \&

Cannon 1999

Mibey \&

Cannon 1999

Spegazzini

1912

Hansford

1957c

Hansford

1961

Hansford

1946

Hansford

1961

Hansford

1961

Hansford

1961 
Myrica sp.

Myrica sp.

Myristicaceae

Horsfieldia gigantifolia*

Horsfieldia glabra

Knema attenuata*

Knema attenuata*

Knema glauca*

Knema glauca*

Myristica fatua (Virola surinamensis)

Myristica sp.

Pycnanthus angolensis (Pycnanthus kombo)

Myrtaceae
Asteridiella myricicola

(Hansf.) Hansf. 1961

Meliola myricicola Hansf.

1947

Asteridiella

myristicacearum Hosag.

2002

Meliola myristicacearum

Hosag. et al. 2014

Meliola uncinata Syd.

1925

Meliola knemicola Hansf.

1954

Asteridiella knemae

(Hansf.) Hansf. 1961

Meliola knemae Hosag. \& P.J. Robin 2011

Asteridiella knemae

(Hansf.) Hansf. 1961

Meliola knemicola Hansf.

1954

Meliola myristicae Hosag.

\& Raghu 1994

!Meliola manoharacharyi

Hosag. et al. 2010

Meliola pycnanthi Hansf.

1957

Asteridiella mammillata

(Hansf.) Hansf. 1961

Meliola helleri Earle 1905

Meliola laxa Gaillard

1892

Meliola pulchella Speg.

1889

Meliola rehmii F. Stevens 1928

Meliola queenslandica (E

Fisher) Hansf. 1953

Asteridiella eucalyptorum

(Hansf.) Hansf. 1961

Asteridiella australiana

(Hansf.) Hansf. 1961

Meliola densa Cooke

1884

Meliola eucalypti $\mathrm{F}$.

Stevens \& Roldan ex

Hansf. 1963

Meliola eugeniae-

calophylloidis Hansf. \&

Deighton 1957

Asteridiella zeyheri

(Doidge) Hansf. 1961

Meliola eugeniae-

monticolae Hansf. 1957

Asteridiella atra(Doidge)

Hansf. 1961

Asteridiella atricha

(Speg.) Hansf. 1961
Hansford

1961

Hansford

1947b

Hosagoudar

2002a

Hosagoudar et

al. 2014

Sydow 1925

Hansford

1954

Hansford

1961

Hosagoudar

\& Robin

2011a

Hansford

1961

Hansford

1954

Hosagoudar et al. 1994b

Hosagoudar et al. 2010a

Hansford

$1956 b$

Hansford

1961

Earle 1904

Gaillard 1892

Index

Fungorum

2016

Stevens 1928

Hansford

1953a

Hansford

1961

Hansford

1961

Cooke 1884

Hansford

1962

Hansford

$1956 \mathrm{~b}$

Hansford

1961

Hansford

$1957 b$

Hansford

1961

Hansford

1961 
Eugenia sp.

Eugenia sp.

Eugenia sp.

Eugenia sp.

Eugenia sp.

Eugenia sp.

Eugenia whytei

Leptospermum brachyandrum

Melaleuca gnidioides

Metrosideros polymorpha

Myrceugenia chequen\#

Myrcia deflexa

Myrcia deflexa

Pimenta racemosa (Amomis caryophyllata)

Psidium guajava

Psidium guajava

Psidium guajava

Psidium guajava

Psidium guajava (Psidium pomiferum)

Syzygium aromaticum

(Eugenia caryophyllata)

Syzygium claviflorum

Syzygium cordatum

Syzygium cumini

Syzygium cumini (Syzygium jambolanum)

Syzygium densiflorum

(Syzygium benthamianum)

Syzygium eucalyptoides

(Eugenia eucalyptoides)

Syzygium fruticosum (Eugenia

fruticosa)

Syzygium grande (Eugenia grandis)

Syzygium guineense
Asteridiella valdiviensis

(Speg.) Hansf. 1961

Meliola arborescens Syd.

\& P. Syd. 1913

Meliola atricha Cif. 1933

Meliola malangasensis

Hansf. 1957

Meliola myrtacearum $\mathrm{F}$.

Stevens \& Roldan ex

Hansf. 1963

Meliola ranganathii

Hansf. 1946

Asteridiella atricha

(Speg.) Hansf. 1961

Meliola leptospermi

Hansf. 1953

Asteridiella melaleucae

B. Huguenin 1969

Amazonia ohiana $\mathrm{F}$.

Stevens 1925

Asteridiella valdiviensis

(Speg.) Hansf. 1961

Irenopsis myrciae Hansf. 1955

Meliola atricha Cif. 1933

Meliola amomicola $\mathrm{F}$.

Stevens 1916

Meliola horrida Ellis \&

Everh. 1893

Meliola olecranonis F.

Stevens \& Tehon 1926

Meliola psidii Fr. 1830

Meliola trichostroma

(Kunze) Toro 1952

Meliola psidii Fr. 1830

Meliola eugeniae Syd.

1922

Amazonia ohiana F.

Stevens 1925

Asteridiella syzygii

(Hansf.) Hansf. 1961

Amazonia syzygii Hosag.

1989

Meliola mollis Berk. \&

Broome 1873

Meliola syzygii-

benthamiani Hosag. \&

T.K. Abraham 1998

Meliola eugeniicola $\mathrm{F}$.

Stevens 1928

Asteridiella eugeniae-

fruticosae Jana et al. 2005

Meliola singaporensis

Hansf. 1946

Asteridiella hughesii

Hansf. 1957
Hansford

1961

Sydow \&

Sydow 1913c

Ciferri 1933

Hansford

$1957 b$

Hansford

1962

Hansford

1946

Hansford

1961

Hansford

$1953 \mathrm{a}$

Index

Fungorum

2016

Stevens 1925

Hansford

1961

Hansford

1955

Ciferri 1933

Stevens 1916

Index

Fungorum

2016

Stevens \&

Tehon 1926

Fries 1830

Toro 1952

Fries 1830

Hansford

1961

Stevens 1925

Hansford

1961

Hosagoudar

\& Goos 1989

Berkeley \&

Broome 1874

Hosagoudar

\& Abraham

1998a

Stevens 1928

Jana et al.

2005a

Hansford

1946

Hansford

$1956 \mathrm{~b}$ 
Syzygium jambos

Syzygium laetum

Syzygium lanceolatum

Syzygium mundagam

Syzygium oblatum (Eugenia oblata)

Syzygium racemosum

(Eugenia jamboloides)

Syzygium sandwicense

(Eugenia sandwicensis)

Syzygium smithii (Acmena smithii var. minor)

Syzygium sp.

Syzygium sp.

Syzygium sp.

Syzygium sp.

Syzygium stocksii (Eugenia stocksii)

Syzygium zeylanicum

Tristania sp.

Nothofagaceae Nothofagus baumanniae

Nyctaginaceae Neea sp.

Pisonia artensis (Calpidia artensis)

Pisonia sp.

Pisonia umbellifera

Ochnaceae

Olacaceae

\section{Gomphia flava (Ouratea} flava)

Ochna atropurpurea

Sauvagesia erecta

Anacolosa sp.

Erythropalum scandens
Meliola eugeniaejamboloidis Hansf. 1954

Hansford

Asteridiella abnormis

(Theiss.) Hansf. 1961

Meliola maduraiensis

Hosag. et al. 1992

Meliola cauveriana

Hosag. et al. 2013

Meliola cylindropoda

Doidge 1920

Meliola eugeniaejamboloidis Hansf. 1954

Meliola hawaiiensis F.

Stevens 1925

Asteridiella acervata

(Ellis \& Everh.) Hansf.

1961

Asteridiella

brahmagiriensis Hosag. et al. 2007

Meliola gersoppaensis

Hosag. et al. 1996

Meliola kukkeensis

Hosag. et al. 2007

Meliola syzygiigena

Hosag. \& Kamar. 2003

Meliola eugeniae-stocksii

Hosag. 1996

Asteridiella atricha

(Speg.) Hansf. 1961

Meliola eugeniae-

jamboloidis Hansf. 1954

Meliola nothofagi B.

Huguenin 1969

Meliola kisubiensis

Hansf. 1947

Meliola calpidiae B.

Huguenin 1969

Meliola pisoniicola $\mathrm{F}$.

Stevens \& Roldan ex

Hansf. 1963

Meliola pisoniae F.

Stevens \& Roldan ex W.

Yamam. 1958

Meliola ourateae Hansf.

\& Deighton 1948

Hansford

1961

Hosagoudar et

al. 1992

Thimmaiah et

al. 2013

Doidge 1920a

Hansford

1954

Stevens 1925

Hansford

1961

Hosagoudar et al. $2007 \mathrm{~b}$

Hosagoudar

1996

Hosagoudar et

al. $2007 \mathrm{~b}$

Hosagoudar

2003b

Hosagoudar

1996

Hansford

1961

Hansford

1954

Index

Fungorum

2016

Hansford

$1947 \mathrm{a}$

Index

Fungorum

2016

Hansford

1962

Yamamoto

1958

Hansford \&

Deighton

1948

Meliola ochnae Doidge Doidge 1920c

1920

Asteridiella ochnacearum Hansford

(Cif.) Hansf. $1961 \quad 1961$

Asteridiella anacolosae Hosagoudar

Hosag. et al. 2011

\& Sabeena

$2011 \mathrm{a}$

Appendiculella

Song et al.

erythropali B. Song et al. $2002 \mathrm{c}$ 
Erythropalum scandens

Octoknema borealis

Olax imbricata (Olax

wightiana)

Olax mannii

Olax obtusifolia

Scorodocarpus borneensis

Strombosia ceylanica

Strombosia scheffleri

Strombosia sp.

Ximenia americana

Ximenia americana

Ximenia americana

Oleaceae
(Erythropalum populifolium)

Meliola erythropali

Hosag. 1990

Meliola octoknemae

Deighton 1951

Meliola olacicola Hosag.

1996

Meliola olacis Deighton

1951

Meliola obtusifoliae

Mibey 1997

Asteridiella scorodocarpi

(Hansf.) Hansf. 1961

Meliola strombosiicola

Hosag. 2013

Asteridiella strombosiae

(Hansf.) Hansf. 1961

Meliola strombosiigena

Hosag. \& Riju 2011

Asteridiella ximeniae

(Bat. \& J. Silva) Hansf.

1961

Irenopsis seyboensis

(Cif.) Hansf. 1961

Irenopsis ximeniae Mibey 1997

!Meliola haploa Cif. 1954

Asteridiella

hispaniolensis (Cif.)

Hansf. 1961

Meliola mayepeae F.

Stevens 1916

(Mayepea domingensis)

Chionanthus ligustrinus

(Mayepea domingensis)

Chionanthus mala-elengi

(Linociera malabarica)

Chionanthus mala-elengi

(Linociera malabarica)

Chionanthus mala-elengi

(Linociera malabarica)

Chionanthus ramiflorus

(Linociera cumingiana)

Chionanthus ramiflorus

(Linociera cumingiana)

Jasminum abyssinicum

Jasminum angulare

Jasminum auriculatum

Jasminum bignoniaceum

Jasminum brevilobum

Jasminum coarctatum

(Jasminum rottlerianum)
Meliola mayepeicola $\mathrm{F}$.

Stevens 1916

Hansf. 1957

Meliola linocierae-

malabaricae Hosag. 1988

Meliola mayepeicola $\mathrm{F}$.

Stevens 1916

Asteridiella linocierae

(Syd. \& P. Syd.) Hansf.

1961

Meliola linocieriicola

Hansf. 1949

Meliola oleicola Doidge

1922

Meliola gemellipoda

Doidge 1922

Meliola jasminicola

Henn. 1895

Meliola jasminigena

Hosag. 2013

Meliola daviesii Hansf.

1946

Meliola daviesii Hansf.

1946
!Asteridiella americana
Hosagoudar

\& Goos 1990a

Deighton

1951

Hosagoudar

1996

Deighton

1951

Mibey \&

Hawksworth

1997

Hansford

1961

Hosagoudar

2013a

Hansford

1961

Hosagoudar

\& Riju 2011b

Hansford

1961

Hansford

1961

Mibey \&

Hawksworth

1997

Ciferri 1954

Hansford

1961

Stevens 1916

Stevens 1916

Farr \&

Rossman

2016

Hosagoudar

$1988 b$

Stevens 1916

Hansford

1961

Hansford

1949

Doidge 1922

Doidge 1922

Hennings

$1895 b$

Hosagoudar

2013b

Hansford

1946

Hansford

1946 
Jasminum dichotomum

Jasminum grandiflorum subsp. floribundum (Jasminum floribundum)

Jasminum pauciflorum

Jasminum sambac

Jasminum sambac

Jasminum sp.

Jasminum sp.

Jasminum streptopus

Jasminum streptopus

Ligustrum perrottetii

Ligustrum perrottetii

(Ligustrum neilgherrense)

Ligustrum robustum subsp. Walkeri (Ligustrum walkeri) Linociera sp.*

Linociera sp.*

Linociera sp.*

Notelaea longifolia (Notelaea reticulata)

Olea capensis (Olea

laurifolia)

Olea capensis (Olea

laurifolia)

Olea dioica

Olea dioica

Olea dioica

Olea dioica

Olea paniculata (Olea glandulifera)

Olea sp.

Osmanthus americanus

Osmanthus cymosus

Osmanthus heterophyllus (Osmanthus aquifolium)
Meliola jasminicola

Henn. 1895

Hennings $1895 b$

Meliola jasmini Hansf. \& Hansford

F. Stevens 1937

1937

Meliola jasminicola

Henn. 1895

Hennings

$1895 b$

Meliola jasminicola

Henn. 1895

Hennings

1895b

Meliola schwarzii Hansf.

Hansford 1954

1954

Meliola busogensis

Hansf. 1938

Hansford

1938

Meliola ngongensis

Hansf. 1947

Hansford

$1947 a$

Meliola capensis

Theissen 1912

(Kalchbr. \& Cooke)

Theiss. 1912

Meliola xumenensis

Doidge 1941

Meliola ligustricola

Hosag. et al. 2005

Irenopsis ligustri M.S.

Patil \& Maham. 1999

Meliola ligustri Hosag. 1990

Asteridiella riethii

Schmied. 1989

Meliola linocierina

Hansf. 1957

Meliola tayabensis H.S.

Yates 1917

Meliola notelaeae Hansf.

1953

Meliola oleicola Doidge 1922

Meliola petiolaris Doidge 1920

!Asteridiella websteri var. Bhise et al. oleae M.R. Bhise \& C.R. 2014 Patil 2014

Asteridiella websteri

Hosag. 1991

Hosagoudar

1991c

Meliola dioicae M.R.

Bhise \& C.R. Patil 2014

Bhise et al.

Meliola oleacearum

Hosag. 2002

Meliola glanduliferae

Hosag. et al. 2005

Meliola malabarensis

Hansf. 1946

!Asteridiella americana

Hansf. 1957

2014

Hosagoudar

2002a

Biju et al.

2005

Hansford

1946

Hansford

$1956 b$

Meliola osmanthi-cymosi

Index

B. Huguenin 1969

Fungorum

2016

Meliola osmanthi Syd. \& P. Syd. 1921
Sydow \&

Sydow 1920a 
Osmanthus heterophyllus

(Osmanthus aquifolium)

Osmanthus sandwicensis

Osmanthus sandwicensis

Osmanthus sandwicensis

Osmanthus sp.

Opiliaceae

Orchidaceae

Pandaceae

Pandanaceae

Passifloraceae
Agonandra brasiliensis

Cansjera rheedii*

Cansjera rheedii*

Cansjera rheedii*

Champereia manillana

Opilia amentacea

Prosthechea cochleata (Epidendrum cochleatum)

Galearia filiformis

Pandanus affinis

Pandanus candelabrum

Pandanus odorifer (Pandanus odoratissimus)

Pandanus sp.

Pandanus sp.

Pandanus sp.

Jaracatia dodecaphylla*

Paropsia sp.

Passiflora sp.

Passiflora sp.

Passiflora sp.

Schlechterina mitostemmatoides

Smeathmannia laevigata
Meliola osmanthi-

aquifolii Hara 1931

Index

Fungorum

2016

Meliola osmanthi Syd. \& Sydow \&

P. Syd. 1921

Meliola osmanthicola

Hansf. 1955

Meliola osmanthina

Hansf. 1955

Meliola osmanthiana

Budathoki \& P.N. Singh

1994

Meliola agonandrae

Speg. 1924

Meliola cansjerae Hansf.

\& Thirum. 1948

Meliola cansjericola

Hosag. 1990

Sydow 1920a

Hansford

1955

Hansford

1955

Budathoki \&

Singh 1994

Spegazzini

1924

Hansford \&

Thirumalacha r 1948

Hosagoudar

\& Goos 1990a

Meliola opiliae Syd. \& P. Sydow 1913

Syd. 1913

Meliola champereiae Syd. Sydow \& \& P. Syd. 1914

Sydow 1914a

Meliola opiliae Syd. \& P. Sydow 1913

Syd. 1913

Meliola orchidacearum Ciferri 1938

Cif. 1938

Meliola galeariae Hansf. Hansford 1954

1954

Meliola pandani Syd. 1928

Meliola pandanicola

Sydow 1928

Hansf. \& Deighton 1948

Hansford \&

Deighton

1948

!Meliola pandani Sawada

\& W. Yamam. 1959

Index

Fungorum

2016

Meliola juttingii Hansf.

1954

Hansford

1954

Meliola kapoorii Hosag.

\& Raghu 1996

Hosagoudar

1996

Meliola pandanacearum

Hosag. \& T.K. Abraham 1999

Hosagoudar

\& Abraham

1999a

Meliola jacaratiae Bat. \&

J.L. Bezerra 1965

Batista et al.

1965a

Meliola paropsiae (Beeli)

Hansf. 1961

Hansford

1961

Irenopsis moelleriana (G. Stevens 1927

Winter) F. Stevens 1927

Irenopsis passiflorae

Hansf. 1955

Hansford

1955

Meliola aristata Toro

1927

Meliola schlechterinae

Mibey 1997

Meliola smeathmanniae

Hansf. \& Deighton 1948
Toro 1927

Mibey \&

Hawksworth 1997

Hansford \&

Deighton

1948 
Turnera ulmifolia

$\begin{array}{ll}\text { Paulowniaceae } & \text { Brandisia } \mathrm{sp} . \\ \text { Pedaliaceae } & \text { Ceratotheca sesamoides }\end{array}$

Sesamum indicum

Sesamum radiatum

Pentaphylacaceae Adinandra bockiana(var. acutifolia)

Eurya chinensis

Pentaphylax euryoides

Ternstroemia toquian*

Ternstroemia vahliana\#

Peraceae Chaetocarpus africanus

Clutia pulchella

Pera citriodora (Pera leandri)

Peridiscaceae Soyauxia floribunda

Phyllanthaceae Actephila excelsa

Antidesma alexiteria

Antidesma alexiteria (Antidesma zeylanicum) Antidesma montanum (Antidesma pentandrum) Antidesma sp.

Antidesma sp.

Antidesma venosum

Aporusa sp.

Bridelia micrantha

Bridelia montana

Bridelia stipularis

Bridelia stipularis

Bridelia stipularis
Asteridiella turneracearum (Cif.)

Hansford

Hansf. 1961

Meliola brandisiae Hansf. Hansford 1948

1948

Meliola sesami Hansf. \& Hansford Deighton 1957

$1956 \mathrm{~b}$

Meliola sesami Hansf. \& Hansford Deighton $1957 \quad 1956 b$

Meliola sesami Hansf. \& Hansford Deighton 1957

!Asteridiella

$1956 \mathrm{~b}$

adinandricola $\mathrm{H}$. Hu 1990

Asteridiella euryae B.

Song \& Y.X. Hu 1996

Meliola pentaphylacis B.

Song \& Y.X. Hu 1997

$\mathrm{Hu} 1990 \mathrm{~b}$

Amazonia toquian Petr. 1955

Meliola ternstroemiicola Hosag. et al. 2014

Song et al.

$1996 b$

Song et al. 1997c

Petrak 1955

Asteridiella entebbeensis

(Hansf. \& F. Stevens)

Hansf. 1961

Meliola cluytiae Van der

Byl 1926

Meliola perae Hansf. Hansford

1955

1955

Asteridiella soyauxiae Deighton

Deighton 1958

1957

Meliola actephilae Hosag. Biju et al. et al. 2005

Asteridiella

antidesmaticola Hosag. \&

2005

Hosagoudar et

T.K. Abraham 1997

Meliola vivekananthanii

Hosag. et al. 2005

Asteridiella antidesmatis

(Petr.) Hansf. 1961

al. 1997

Biju et al.

2005

Hansford

1961

Meliola luzonensis Syd. \& Sydow \&

P. Syd. 1917

Meliola mauritiana

Hansf. 1955

Sydow 1917 a

Hansford

1955

Asteridiella antidesmatis

(Petr.) Hansf. 1961

Hansford

1961

Meliola aporusae Hosag.

\& P.J. Robin 2011

Hosagoudar

\& Robin

2011a

Meliola brideliicola

Hansford

Hansf. 1946

1946

Meliola himalayensis J.N. Index

Kapoor 1967

Fungorum

2016

!Meliola brideliae F.

Stevens \&

Stevens \& Roldan 1935 Roldan 1935

!Meliola brideliae Hansf.

1946

Hansford

1946

Meliola brideliae F.

Hansford

Stevens \& Roldan ex

Hansf. 1963
1962 
Cleistanthus sp.

Glochidion heyneanum

(Glochidion velutinum)

Glochidion lutescens

(Glochidion hypoleucum)

Glochidion sp.

Glochidion sp.

Glochidion sp.

Glochidion sp.

Glochidion sp.

Hymenocardia lyrata

Maesobotrya barteri var. sparsiflora (Maesobotrya sparsiflora)

Margaritaria discoidea

(Phyllanthus discoideus)

Phyllanthus albus (Glochidion album)

Phyllanthus profusus

(Phyllanthus wildemannii)

Phyllanthus reticulatus

Phyllanthus sp.

Sauropus androgynus

Sauropus sp.

Uapaca sp.

Phytolaccaceae Petiveria alliacea

Phytolacca dininae\#

Phytolacca dioica

Phytolacca dodecandra

Picramniaceae Picramnia antidesma

Picrodendraceae Oldfieldia africana

Petalostigma quadriloculare

Pinaceae
Abies sp.
Asteridiella cleistanthi

Hansf. 1958

Meliola glochidii $\mathrm{F}$.

Stevens \& Roldan ex

Hansf. 1963

Meliola glochidiicola W. Yamamoto

Yamam. 1941

\#Irenina sinuosa $\mathrm{F}$.

Stevens \& Roldan 1935

Asteridiella entebbeensis

(Hansf. \& F. Stevens)

Hansf. 1961

Meliola glochidiifolia

Hosag. 2013

Meliola karnatakensis

Hosag. et al. 1996

Meliola thiteana Hosag.

1996

Meliola hymenocardiae

Hansf. \& Deighton 1948

Meliola maesobotryae

Hansf. \& Deighton 1948

Asteridiella entebbeensis

(Hansf. \& F. Stevens)

Hansf. 1961

Meliola kansireiensis W.

Yamam. 1941

Asteridiella phyllanthi

(Deighton) Hansf. 1961

Meliola phyllanthicola

Hansf. \& Deighton 1957

Meliola phyllanthigena

Hosag. 2013

Asteridiella entebbeensis

(Hansf. \& F. Stevens)

Hansf. 1961

Meliola sauropicola H.S.

Yates 1917

Appendiculella

uapacicola (S. Hughes)

Hansf. 1961

Meliola petiveriae Hansf. Hansford

1963

Meliola incompta Syd. \&

P. Syd. 1920

Meliola phytolaccae-

dioicae Hansf. 1957

Meliola phytolaccae

Hansf. \& F. Stevens 1937

Meliola picramniae

Hansf. 1957

Meliola homalanthi

Boedijn 1940

Meliola petalostigmatis

Hansf. 1953

Meliola abietis Sacc.

1882
1962

Hansford

$1957 \mathrm{c}$

Hansford

1962

1941a

Stevens \&

Roldan 1935

Hansford

1961

Hosagoudar

2013b

Hosagoudar

1996

Hosagoudar

1996

Hansford \&

Deighton

1948

Hansford \&

Deighton

1948

Hansford

1961

Yamamoto

1941a

Hansford

1961

Hansford

$1956 \mathrm{~b}$

Hosagoudar

2013b

Hansford

1961

Yates 1917

Hansford

1961

Sydow \&

Sydow 1920b

Hansford

1957b

Hansford

1937

Hansford

1957b

Boedijn 1940

Hansford

1953a

Saccardo

1882 
Pinus echinata

Pinus sp.

Piperaceae

Piper aduncum

Piper aduncum

Piper barbatum

Piper blattarum

Piper bogotense (Piper

tungurahuae)

Piper carrilloanum (Piper

paulownifolium)

Piper guineense

Piper guineense

Piper hispidum

Piper nigrum

Piper samanense*

Piper scabrum\#

Pipersp.

Piper sp.

Piper sp.

Piper sp.

Piper sp.

Piper sp.

Piper tucumanum

Pittosporaceae Pittosporum brackenridgei*

Pittosporum neelgherrense*

Pittosporum pentandrum

Pittosporum senacia

Pittosporum sp.

Pittosporum undulatum
Asteridiella pinicola

(Dearn.) Hansf. 1961

Asteridiella pinicola

(Dearn.) Hansf. 1961

Amazonia asterinoides

(G. Winter) Theiss. 1913

Asteridiella obducens

(Gaillard) Hansf. 1961

Irenopsis moelleriana (G. Stevens 1927

Winter) F. Stevens 1927

Meliola gaillardiana $\mathrm{F}$.

Stevens 1916

Meliola piperis Earle

1901

Meliola piperis-barbati

Hansf. 1956

Hansford

1961

Hansford

1961

Theissen 1913

Hansford

1961

Meliola paucipes $\mathrm{F}$.

Stevens 1916

Meliola microthea Syd.

1939

Meliola zetekii F. Stevens

1928

Amazonia ugandensis Hansford

Hansf. 1937

1937

Asteridiella pipericola

(Hansf.) Hansf. 1961

Hansford

1961

Meliola contorta $\mathrm{F}$

Stevens 1916

Stevens 1916

Meliola thitei Hosag. Hosagoudar

1996

1996

Meliola gaillardiana F. Stevens 1916

Stevens 1916

Amazonia pipericola Bat. Maia \&

\& Taltasse 1960

Batista 1960

Amazonia piperis (Syd. \& Hansford

P. Syd.) Hansf. 1955

1955

Meliola patouillardii

Gaillard 1892

Gaillard 1892

Meliola piperina Syd. \&

P. Syd. 1916

Meliola pululahuensis

Gaillard 1892

Meliola stenospora $\mathrm{G}$. Winter 1886

Winter 1886

Meliola tumatumariensis Hansford

Hansf. 1957

1957b

Meliola singeri Petr. 1963 Petrak 1962

Meliola polytricha

Kalchbr. \& Cooke 1879

Asteridiella

Cooke 1879

pittosporacearum Hosag. \& Sabeena

\& Sabeena 2012

2012

Meliola elmeri Syd. \& P.

Syd. 1912

Meliola pittospori Hansf.

1955

Sydow \&

Sydow 1912b

Hansford

1955

Meliola amphitricha Fr. Fries 1828

1828

Meliola amaniensis

Hansf. 1946
Hansford

1946 
Plantaginaceae

Lophospermum erubescens (Maurandya erubescens)

Plantago sp.

Plumbaginaceae Plumbago sp.

Poaceae

\section{?Anthistiria sp.* \\ Aristida gibbosa (Aristida marginalis)}

Arundinaria sp.

Arundo donax

Bambusa sp.

Bambusa sp.

Bambusa sp.

Capillipedium sp.

Chrysopogon zizanioides (Vetiveria zizanioides) Cymbopogon nardus

Cyrtococcum longipes Imperata cylindrica

Lasiacis divaricata

Lasiacis maculata

Lasiacis maculata (Lasiacis sorghoidea)

Lasiacis maculata (Lasiacis swartziana)

Lasiacis oaxacensis

Lasiacis procerrima

Lasiacis rudifolia

Lasiacis ruscifolia

Lasiacis ruscifolia (Lasiacis compacta)

Olyra latifolia

Ophiuros exaltatus

(Rottboellia exaltata)

Panicum hallii (Panicum

filipes)

Panicum latifolium
Meliola maurandiae

Hansf. 1957

Meliola plantaginis

Hansf. \& F. Stevens 1937

Meliola plumbaginis

Hansf. \& F. Stevens 1937

Meliola boedijniana

Hansf. 1954

Meliola hercules Höhn. 1909

Meliola aristidae Bat. \&

J. Silva 1953

Meliola tenuis Berk. \&

M.A. Curtis 1878

Meliola arundinis Pat. 1897

Meliola bambusae Pat. 1888

Meliola bambusicola

Hansf. 1947

Meliola phyllostachydis

W. Yamam. 1941

Meliola capillipedii Jana et al. 2005

Meliola panici Earle 1901

Meliola cymbopogonis

J.N. Kapoor 1967

Meliola panici Earle 1901

Meliola imperatae Syd. \& P. Syd. 1917

Meliola lasiacidis Toro 1934

Meliola lasiacidis Toro 1934

Meliola lasiacidis Toro 1934

Meliola lasiacidis Toro 1934

Meliola lasiacidis Toro 1934

Meliola lasiacidis Toro 1934

Meliola lasiacidis Toro 1934

Meliola lasiacidis Toro 1934

Meliola lasiacidis Toro 1934 Meliola panici Earle 1901

Meliola substenospora Höhn. 1909

Meliola panicicola Syd. \& P. Syd. 1914 Meliola panici Earle 1901
Hansford 1957b

Hansford 1937

Hansford

1937

Hansford

1954

Höhnel 1909a

Index

Fungorum

2016

Index

Fungorum

2016

Patouillard

1897

Index

Fungorum

2016

Hansford

1947a

Yamamoto

1941a

Jana et al.

2005d

Earle 1901

Index

Fungorum

2016

Earle 1901

Sydow \&

Sydow 1917a

Chardón \&

Toro 1934

Chardón \&

Toro 1934

Chardón \&

Toro 1934

Chardón \&

Toro 1934

Chardón \&

Toro 1934

Chardón \&

Toro 1934

Chardón \&

Toro 1934

Chardón \&

Toro 1934

Chardón \&

Toro 1934

Earle 1901

Höhnel 1909a

Sydow \&

Sydow 1914a

Earle 1901 
Panicum sp.

Pharus sp.

Phragmites karka

Phragmites sp.

Phragmites sp.

Pseudosasa usawai*

Saccharum spontaneum

Sasa borealis

Setaria megaphylla (Setaria chevalieri)

Setaria palmifolia

Setaria palmifolia

(Chaetochloa sulcata)

Setaria palmifolia (Panicum palmifolium)

Setaria sp.

Sorghum halepense (Andropogon halepensis)

Spartina cynosuroides (Spartina polystachya)

Stenotaphrum secundatum

Stipa dregeana

Stipa dregeana

Themeda cymbaria

Themeda gigantea

Themeda triandra

Uniola virgata

Yushania alpina (Arundinaria alpina)

Podocarpaceae Nageia nagi (Podocarpus nagi)

Podocarpus coriaceus

Podocarpus elongatus

Podocarpus latifolius (Podocarpus thunbergii)
Meliola panici Earle $1901 \quad$ Earle 1901

Meliola combinans Cif. Ciferri 1956 1957

Meliola dolabrata Syd. \& Sydow \& P. Syd. $1921 \quad$ Sydow 1921

Meliola arundinis Pat. Patouillard 1897

1897

Meliola substenospora Höhnel 1909a

Höhn. 1909

Meliola phyllostachydis Yamamoto

W. Yamam. $1941 \quad 1941 \mathrm{a}$

Meliola sacchari Syd. \& Sydow \&

P. Syd. 1914

Meliola pseudosasae I. Hino \&

Hino 1958

Katumoto

1958

Meliola setariae Hansf. \& Hansford \& Deighton 1948

Deighton

1948

Meliola panici Earle 1901 Earle 1901

Meliola chaetochloae F. Stevens 1928

Stevens 1928

Meliola panicicola Syd. $\quad$ Sydow \&

\& P. Syd. $1914 \quad$ Sydow 1914a

Meliola setariae Hansf. \& Hansford \&

Deighton 1948

Deighton

1948

Meliola andropogonis F. Yamamoto

Stevens \& A. Roldán ex 1958

W. Yamam. 1958

Meliola spartinae (Ellis \& Berlese \&

Everh.) Berl. \& Voglino Voglino 1886

1886

Meliola stenotaphri F. Stevens 1916

Stevens 1916

!Meliola stipae Mibey Mibey \&

2001

Kokwaro

2001

Meliola panici Earle 1901 Earle 1901

Meliola themedae F.

Petrak 1962

Stevens \& Roldan ex

Hansf. 1963

Meliola themedae F.

Stevens \& Roldan ex

Hansf. 1963

Meliola themedicola

Hosag. et al. 2005

Petrak 1962

Meliola panici Earle 1901

!Meliola arundinariae

Mibey 2001

Meliola podocarpicola

Y.X. Hu \& B. Song 1992

Asteridiella podocarpi

(Doidge) Hansf. 1961

Asteridiella podocarpi

(Doidge) Hansf. 1961

Asteridiella podocarpi

(Doidge) Hansf. 1961
Biju et al. 2005

Earle 1901

Mibey \&

Kokwaro

2001

Hu et al. 1992

Hansford

1961

Hansford

1961

Hansford

1961 
Podocarpus latifolius

(Podocarpus thunbergii)

Podocarpus sp.

Polemoniaceae

Allophyllum erosi\#

Allophyllum sp.

Polygalaceae Carpolobia alba

Carpolobia lutea

Monnina celastroides

(Monnina rupestris)

Polygala arillata

Securidaca sp.

Securidaca sp.

Securidaca volubilis

Securidaca welwitschii

Xanthophyllum flavescens

Polygonaceae Coccoloba ?buchii

Coccoloba caracasana

Coccoloba caracasana

?Coccoloba costata

Coccoloba diversifolia

(Coccoloba laurifolia)

Coccoloba diversifolia

(Coccoloba laurifolia)

Coccoloba nodosa

Coccoloba olivascens\#

Coccoloba sp.

Coccoloba sp.

Leptogonum domingense var. molle (Leptogonum molle)

Leptogonum domingense*

Persicaria chinensis

(Polygonum chinense)

Persicaria chinensis

(Polygonum chinense)

Persicaria chinensis

(Polygonum chinense)
Meliola peltata Doidge

1917

Asteridiella podocarpi Hansford

(Doidge) Hansf. $1961 \quad 1961$

Meliola allophyli Doidge Doidge \&

1928

Meliola tijucensis Hansf.

1955

Meliola carpolobiae

Hansf. 1946

Meliola carpolobiicola

Hansf. \& Deighton 1948

Asteridiella monninae $(\mathrm{F}$.

Stevens) Hansf. 1961

Meliola thiyagesanii

Hosag. et al. 2010

!Meliola securidacae var.

vanderystii Hansf. 1957

Meliola kisantuensis

Hansf. 1957

Meliola securidacicola

Hansf. 1961

Asteridiella securidacae

(Hansf.) Hansf. 1961

Irenopsis xanthophylli

Hosag. 1991

Asteridiella

montecristensis Hansf.

1957

Meliola angusta $\mathrm{F}$.

Stevens \& Tehon 1926

Meliola solteroi Hansf.

1957

Meliola stevensiana Cif.

1954

Irenopsis rectangularis

(F. Stevens) F. Stevens 1927

Meliola panamensis $\mathrm{F}$.

Stevens 1928

Meliola coccolobae-

nodosae Hansf. 1955

Meliola macracantha

(Cif.) Hansf. 1961

Meliola coccolobis F.

Stevens \& Tehon 1926

Meliola praetervisa

Gaillard 1892

Meliola biserrata (Cif.)

Cif. 1961

Meliola leptogoni (Cif.)

Cif. 1961

Asteridiella polygoni

(Hansf.) Hansf. 1961

Meliola polygoni

Srinivas. 1974

Meliola polygonicola

Hosag. 1996
Sydow 1928

Hansford

1955

Hansford

1946

Hansford \&

Deighton

1948

Hansford

1961

Nithyatharani

et al. 2010

Hansford

1957b

Hansford

1957b

Hansford

1961

Hansford

1961

Hosagoudar

\& Goos 1991

Hansford

$1956 b$

Stevens \&

Tehon 1926

Hansford

$1956 \mathrm{~b}$

Ciferri 1954

Stevens 1927

Stevens 1928

Hansford

1955

Hansford

1961

Stevens \&

Tehon 1926

Gaillard 1892

Hansford

1961

Hansford

1961

Hansford

1961

Srinivasulu

1974

Hosagoudar

1996 
Ardisia guadeloupensis

Ardisia kivuensis (Afrardisia dentata)

Ardisia missionis

Ardisia perrottetiana

Ardisia polysticta (Ardisia jagorii)

Ardisia sp.

Cybianthus sp.

Discocalyx cybianthoides

Jacquinia eggersii var. intermedia*

Maesa elmeri*

Maesa indica

Maesa lanceolata

Maesa lanceolata var. rufescens (Maesa rufescens) Maesa lanceolata var. rufescens (Maesa rufescens) Maesa laxa*

Maesa laxa*

Maesa sp.

Maesa sp.

Myrsine africana

Myrsine seguinii (Rapanea neriifolia)

Myrsine sp.

Myrsine sp.

Myrsine sp.

Myrsine sp.

Myrsine sp.

Parathesis serrulata
Amazonia antistrophicola Hosag. \& T.K. Abraham 1998

Hosagoudar \& Abraham 1998a

Meliola myrsinacearum

Stevens 1916

F. Stevens 1916

Meliola transvaalensis $\quad$ Doidge 1927

Doidge 1927

Meliola ardisiicola

Hosag. et al. 2004

Hosagoudar

2004b

Meliola groteana Syd. \& P. Syd. 1913

Sydow \&

Meliola ardisiae Syd. 1925

Meliola ardisiigena

Hosag. et al. 2011

Meliola cybianthis Toro

1934

Meliola discocalycis $\mathrm{F}$.

Stevens ex Hansf. 1957

Meliola agnolae-mariae

Cif. 1954

Amazonia peregrina (Syd. Sydow \& \& P. Syd.) Syd. \& P. Syd. Sydow 1917a 1917

Meliola groteana Syd. \& Sydow \& P. Syd. 1913

Sydow 1913d

Meliola groteana Syd. \& Sydow \&

P. Syd. 1913

Sydow 1913d

Meliola biegensis Hansf. 1957

Hansford

$1957 b$

Meliola furcillata Doidge Doidge 1917

1917

Amazonia peregrina (Syd. Sydow \&

\& P. Syd.) Syd. \& P. Syd. Sydow 1917a 1917

Meliola maesae Rehm

1913

Meliola fructicola Hansf. Hansford

1957

$1956 b$

Meliola maesicola Hansf. Hansford

\& F. Stevens 1937

Meliola transvaalensis $\quad$ Doidge 1927

Doidge 1927

Meliola australis I. Hino Hino \&

\& Katum. 1957

Katumoto

1957

Asteridiella theisseniana Hansford

Hansf. 1957

$1956 b$

Irenopsis armata (Speg.) Stevens 1927

F. Stevens 1927

Meliola cladotricha Lév. Léveillé 1846

1846

Meliola delicatula Speg.

1888

Spegazzini

$1888 b$

Meliola quercinopsis

Rehm 1901

Rehm 1901

Asteridiella parathesiana

(Cif.) Hansf. 1961
Hansford

1961 
Parathesis serrulata

Petesiodes clusiifolium

(Wallenia laurifolia)

Rapanea umbellulata

Rapanea wightiana

Suttonia sp.

Wallenia apiculata*

Wallenia sp.

Proteaceae

Banksia dentata

Grevillea gillivrayi

Grevillea robusta

Grevillea robusta

Helicia cochinchinensis

Helicia formosana

Helicia sp.

Knightia excelsa*

Lomatia arborescens*

Lomatia fraseri*

Lomatia hirsuta (Lomatia obliqua )

Lomatia hirsuta (Lomatia obliqua )

Lomatia hirsuta (Lomatia obliqua )

Lomatia hirsuta (Lomatia obliqua )

Lomatia myricoides*

Lomatia silaifolia

Orites excelsus*

Roupala montana (Roupala gardneri)

Stenocarpus salignus*

Stenocarpus umbellifer

Telopea oreades*
Irenopsis parathesicola

Stevens 1927

(F. Stevens) F. Stevens

1927

Asteridiella miriapoda Hansford

(Cif.) Hansf. $1963 \quad 1962$

Meliola rapaneae Syd. $\quad$ Sydow 1928

1928

Meliola rapaneae Syd. Sydow 1928

1928

Amazonia suttoniae (F. Hansford

Stevens) Hansf. $1955 \quad 1955$

Meliola sphaeropoda Cif. Ciferri 1938 1938

Meliola yaquensis Petr. \& Petrak \&

Cif. $1932 \quad$ Ciferri 1932

Meliola banksiae Hansf. Hansford

1958

Meliola grevilleae-

$1957 \mathrm{c}$

gillivrayi B. Huguenin Fungorum

1969

2016

Meliola funerea

McAlpine

McAlpine 1896

1896

Meliola grevilleae Hansf. Hansford 1958

$1957 \mathrm{c}$

Asteridiella heliciae B. Song \& Li

Song \& T.H. Li $2004 \quad 2004$ b

Meliola heliciae W. Yamamoto

Yamam. 1941

$1941 b$

Meliola heliciicola Hansf. Hansford

1958

$1957 \mathrm{c}$

Asteridiella knightiae S. Hughes 1978

Hughes 1978

Meliola funerea

McAlpine 1896

Meliola funerea

McAlpine 1896

Meliola funerea

McAlpine 1896

Meliola funerea

McAlpine 1896

Meliola lanosa Pat. 1888

McAlpine

1896

McAlpine

1896

McAlpine

1896

McAlpine

1896

Doassans \&

Patouillard

1888

Meliola negeriana Syd. \& Sydow \&

P. Syd. 1904

Meliola funerea

McAlpine 1896

Meliola funerea

McAlpine 1896

Meliola funerea

McAlpine 1896

Sydow 1904

McAlpine

1896

McAlpine

1896

McAlpine

1896

Irenopsis rupalae (Speg.) Stevens 1927

F. Stevens 1927

Meliola funerea

McAlpine 1896

McAlpine

1896

Meliola stenocarpi $\mathrm{B}$.

Huguenin 1969

Meliola funerea

McAlpine 1896
Index

Fungorum

2016

McAlpine

1896 


$\begin{array}{ll}\text { Pteridaceae } & \begin{array}{l}\text { Adiantum latifolium } \\ \text { Putranjivaceae }\end{array} \\ & \begin{array}{l}\text { Drypetes longifolia (Drypetes } \\ \text { macrophylla) } \\ \text { Drypetes sp. }\end{array} \\ & \begin{array}{l}\text { Drypetes venusta (Drypetes } \\ \text { elata) } \\ \text { Anemone vesicatoria } \\ \text { (Knowltonia vesicatoria) } \\ \text { Coptis trifolia }\end{array}\end{array}$

Rhamnaceae

\section{Alphitonia excelsa \\ Colletia ?spinosissima \\ (Colletia ?spinosa) \\ Colubrina rufa* \\ Colubrina travancorica}

Condalia hookeri (Condalia obovata*)

Emmenosperma alphitonoides

Gouania leptostachya

Gouania longispicata

Gouania lupuloides

Gouania microcarpa

Gouania microcarpa

Gouania sp.

Gouania sp.

Hovenia acerba

Hovenia dulcis

Krugiodendron ferreum

Maesopsis eminii

Pomaderris apetala*

Scutia arenicola

Scutia buxifolia

Scutia myrtina
Irenopsis pteridicola $(\mathrm{F}$. Stevens) Hansf. 1955

Meliola drypeticola

Hosag. 1990

Asteridiella drypeticola

Hansf. 1957

Meliola erumeliensis

Hosag. et al. 2009

Meliola knowltoniae

Doidge 1924

Appendiculella alpina

(Togashi \& Mentzer)

Katum. 2010

Irenopsis aciculosa $(\mathrm{G}$.

Winter) F. Stevens 1927

Appendiculella splendens

(F. Stevens) Hansf. 1961

Meliola colletiae Hansf.

1961

Asteridiella colubrinae

(F. Stevens) Hansf. 1961

Meliola colubrinicola

Hosag. \& Sabeena 2012

Meliola condaliae F.

Stevens 1946

Meliola emmenospermatis

Hansf. 1953

Irenopsis tenuissima $(\mathrm{F}$.

Stevens) F. Stevens 1927

Meliola gouaniae Hansf.

1945

Irenopsis tenuissima $(\mathrm{F}$.

Stevens) F. Stevens 1927

Amazonia gouaniae

Hosag. \& U. Braun 1989

Meliola gouaniicola

Hosag. \& P.J. Robin 2011

!Meliola gouaniae var. keralica Hosag. \& P.J.

Robin 2011

Meliola rhamnicola $\mathrm{F}$.

Stevens \& Tehon 1926

Meliola hoveniae Hosag. et al. 2011

Appendiculella hoveniae

A.K. Kar \& Maity 1971

Meliola krugiodendri Cif.

1938

Meliola maesopsidis

Hansf. 1947

Meliola pomaderridis

Hansf. 1946

Meliola tridentata Hansf.

1955

Meliola scutiae Speg.

1912

Asteridiella emciciana

Hosag. et al. 2009
Hansford

1955

Hosagoudar

\& Goos 1990a

Hansford

$1956 b$

Hosagoudar et

al. 2009a

Doidge 1924

Katumoto

2010

Stevens 1927

Hansford

1961

Hansford

1961

Hansford

1961

Hosagoudar

\& Sabeena

2012

Stevenson

1946

Hansford

1953a

Stevens 1927

Hansford

1945 a

Stevens 1927

Hosagoudar

\& Braun 1989

Hosagoudar

\& Robin

2011a

Hosagoudar

$\&$ Robin

2011b

Stevens \&

Tehon 1926

Nithyatharani

et al. 2011

Kar \& Maity

$1970 \mathrm{a}$

Ciferri 1938

Hansford

1947a

Hansford

1946

Hansford

1955

Spegazzini

1912

Hosagoudar et al. 2009c 
Scutia myrtina

Ventilago africana

Ventilago elegans

Ziziphus rugosa

Rhipogonaceae Rhipogonum album

Rhizophoraceae Bruguiera sexangula (Bruguiera eriopetala)

Carallia brachiata

Ceriops tagal

Rosaceae

Acaena sp.

Geum chilense*

Heteromeles sp.

Photinia beauverdiana (Photinia kudoi)

?Photinia sp.

Prunus africana (Pygeum africanum)

Prunus sp.

Prunus sp. (Pygeum sp.)

Prunus subcoriacea*

Pyracantha crenulata

Pyracantha crenulata

Pyracantha crenulata

(Crataegus crenulata)

Rhaphiolepis indica

Rosa roxburghii

Rubus buergeri (Rubus moluccanus)

Rubus buergeri (Rubus moluccanus)

Rubus buergeri (Rubus moluccanus)
Irenopsis scutiae Hansf. 1946

Meliola ventilaginicola

Hansf. 1946

Meliola ventilaginis $\mathrm{W}$.

Yamam. 1941

Meliola ziziphi Hansf. \&

Thirum. 1948

Meliola ripogoni Hansf. 1953

Hansford

1946

Hansford

1946

Yamamoto

1941a

Hansford \&

Thirumalacha r 1948

Hansford

Meliola brugnierae Syd. Sydow 1925 1925

Meliola anisophylleae Hansford \&

Hansf. \& Deighton 1948 Deighton

1948

Meliola ceriopis Hansf. Hansford 1957

$1956 b$

Appendiculella acaenae Hansford

Hansf. 1955

1955

Meliola autumnalis Syd. $\quad$ Sydow \&

\& P. Syd. 1904

Sydow 1904

Meliola heteromeles Berlese \&

(Cooke \& Harkn.) Berl. \& Voglino 1886

Voglino 1886

Appendiculella Hansford

photiniicola (W. Yamam.) 1961

Hansf. 1961

Asteridiella glabriuscula Hansford

(Speg.) Hansf. $1961 \quad 1961$

Asteridiella pygei Hansf. Hansford

1957

$1957 b$

Asteridiella prunicola Hansford

(Speg.) Hansf. 1961

Meliola pygeicola Hosag. Hosagoudar

2013

2013 b

Asteridiella prunicola Hansford

(Speg.) Hansf. 1961

Appendiculella

pyracanthae R.K.

Chaudhary et al. 1994

Meliola garhwalensis S.L. Srivastava \&

Srivast. \& Topal $1981 \quad$ Topal 1981

Irenopsis crataegi S.K. Index

Bose 1962

Asteridiella

rhaphiolepidis (W.

Yamam.) Hansf. 1961

Asteridiella rosae

(Hansf.) Hansf. 1961

Chaudhary et

al. 1994

Fungorum

2016

Hansford

1961

Hansford

1961

!Meliola rubi $\mathrm{F}$. Stevens Hansford

\& Roldan ex Hansf. 19631962

\#Irenina rubi var.

Ciferri 1954

angulata $\mathrm{F}$. Stevens \&

Roldan 1935

Irenopsis rubi M.S. Patil Patil \&

\& Maham. 1999
Mahamulkar

1999 
Rubus buergeri (Rubus moluccanus)

Rubus formosensis

Rubus fruticosus\#

Rubus keniensis*

Rubus niveus

Rubus rosifolius

Rubus rosifolius (Rubus rosaefolius)

Rubus rosifolius (Rubus rosaefolius)

Rubus rosifolius (Rubus rosaefolius)

Rubus sp.

Rubus sp.

Rubus sp.

Stranvaesia davidiana var. davidiana* (Stranvaesia niitakayamensis)

Rubiaceae

\section{Adina sp.}

Aidia genipiflora (Randia genipiflora)
Meliola rubiella Hansf. 1957

Hansford

Meliola formosensis W.

Yamam. 1941

Appendiculella calostroma (Desm.)

Höhn. 1919

Appendiculella rubi

Mibey 1996

$1957 b$

Yamamoto

1941a

Höhnel 1919

Meliola rubiella Hansf. 1957

Meliola rubicola Henn.

1904

\#Irenina rubi F. Stevens

\& Roldan 1935

\#Irenina rubi var.

angulata F. Stevens \&

Roldan 1935

Asteridiella rubi $\mathrm{F}$.

Stevens \& Roldan ex

Hansf. 1963

Asteridiella duportii

Hansf. 1961

Asteridiella manca (Ellis

\& G. Martin) Hansf. 1961

Asteridiella pygei Hansf.

1957

Appendiculella

stranvaesiicola (W.

Yamam.) Hansf. 1961

\#Meliola woodiana var.

aristata Hansf. 1937

Amazonia rubiacearum

Mibey 1997

Meliola bwaniana Mibey 1997

Mibey et al.

1996

Hansford

$1957 \mathrm{~b}$

Hennings

1904a

Ciferri 1954

Ciferri 1954

Hansford

1962

Hansford

1961

Hansford

1961

Hansford

$1957 b$

Hansford

1961

Hansford

1937

Mibey \&

Hawksworth

1997

Mibey \&

Hawksworth

1997

Meliola coorgiana Hosag. et al. 2013

Meliola darwiniana

Mibey 1997

Thimmaiah et

al. 2013

Mibey \&

Hawksworth

1997

Meliola intermedia

Gaillard 1892

Meliola rubiacearum

Mibey 1997

Gaillard 1892

Mibey \&

Hawksworth

1997

Meliola smallii Hansf. \&

Hansford

F. Stevens 1937

1937

Meliola spirobelia Bat. \&

M.P. Herrera 1965

Meliola tawaoensis

Hansf. 1957

Meliola bonarii Bat. \&

Nascim. 1957

Batista et al.

1965a

Hansford

$1956 b$

Batista \&

Maia 1957

Meliola randiae Hansf. \& Hansford \&

Deighton 1948
Deighton

1948 
Aidia genipiflora (Randia genipiflora)

Alibertia edulis

Anthospermum sp.

Bathysa australis

Burchellia bubalina

(Burchellia capensis)

Canthium angustifolium

Canthium monstrosum

(Plectronia monstrosa)

Canthium rheedei

Canthium rheedei

Canthium sp.

Canthium sp.

Canthium sp.

Chassalia abrupta\#

Chassalia discolor

Chassalia kolly (Psychotria warneckei)

Chassalia $\mathrm{sp.}$

Chiococca alba

Chiococca alba

Coccocypselum guianense

(Tontanea guianensis)

Coccocypselum herbaceum

(Coccocypselum repens)

Coccocypselum sp.

Coffea ?canephora

(Coffea ?robusta)

Coffea canephora (Coffea

robusta)

Coffea liberica

Coffea liberica var. dewevrei

(Coffea excelsa)

Coffea sp.
Meliola tunkiaensis

Hansf. \& Deighton 1948

Meliola alibertiae $\mathrm{F}$.

Stevens 1928

Meliola anthospermi Hansford

Hansf. 1947

Meliola thalliformis

Deighton 1958

Meliola litoralis Syd. 1928

Meliola canthii-

angustifolii Hosag. 1996

Asteridiella tarlacensis

Petr. 1959

Meliola canthiicola

Hosag. et al. 2001

Meliola henryi Hosag.

1996

Amazonia goosii Hosag.

\& T.K. Abraham 1998

Asteridiella glabra (Berk.

\& M.A. Curtis) Hansf.

1961

Meliola canthiigena

Hosag. et al. 2007

Meliola chasaliae Mibey 1997

Meliola taitensis Mibey \& Mibey \&

P.F. Cannon 1999

Meliola imperspicua

Deighton 1957

Meliola chassaliicola

Hosag. 2004

1947a

Deighton

1957

Doidge \&

Sydow 1928

Hosagoudar

1996

Petrak 1958

Hosagoudar et al. $2001 \mathrm{~b}$

Hosagoudar

1996

Hosagoudar

\& Abraham

$1998 \mathrm{a}$

Hansford

1961

Hosagoudar et al. $2007 \mathrm{~b}$

Mibey \&

Hawksworth

1997

Cannon 1999

Deighton

1957

Hosagoudar

$2004 \mathrm{~b}$

Irenopsis chiococcae (F. Stevens 1927

Stevens) F. Stevens 1927

Meliola psychotriae Earle Earle 1904

1905

Meliola amphigena $\mathrm{F}$.

Stevens \& Tehon 1926

Stevens \&

Tehon 1926

Asteridiella glabra (Berk. Hansford

\& M.A. Curtis) Hansf. 1961

1961

Asteridiella seminata

(Berk. \& M.A. Curtis)

Hansf. 1961

Meliola ghesquierei

Hansf. 1957

Hansford

1961

Hansford

$1957 b$

Meliola coffeae Hansf. Hansford

1937

1937

Asteridiella naucleae Hansford

(Boedijn) Hansf. 1961

1961

Asteridiella glabra (Berk. Hansford

\& M.A. Curtis) Hansf.

1961

Asteridiella glabra (Berk. \& M.A. Curtis) Hansf.

1961
1961

Hansford

1961 
Coffea stenophylla

Coprosma robusta

Coprosma sp.

Craterispermum

schweinfurthii

Cuviera acutiflora

Erithalis fruticosa (Erithalis parviflora)

Evea sp.*

Exostema sanctae-luciae

Feretia sp.

Fleroya stipulosa (Mitragyna stipulosa)

Fleroya stipulosa (Mitragyna

stipulosa)

Gaertnera paniculata

Galopina circaeoides

Galopina circaeoides

Gonzalagunia hirsuta

(Gonzalagunia spicata)

Gonzalagunia panamensis

(Duggena panamensis)

Gonzalagunia rudis (Duggena

rudis)

Guettarda pungens

Guettarda sp.

Guettarda sp.

Hedyotis albonerva

Hedyotis leschenaultiana

(Hedyotis stylosa)

Hedyotis sp.

Hymenodictyon obovatum

Hypobathrum racemosum

(Petunga roxburghii)

Isertia haenkeana

Ixora coccinea

Ixora coccinea
Meliola psychotriae Earle Earle 1904 1905

Endomeliola dingleyae S. Hughes \&

Hughes \& Piroz. $1994 \quad$ Pirozynski

1994

Asteridiella coprosmae

Hansf. 1957

Meliola craterispermi

Mibey \& P.F. Cannon

1999

Asteridiella naucleae

(Boedijn) Hansf. 1961

Meliola erithalidis (Cif.)

Hansf. 1949

Hansford

$1957 b$

Mibey \&

Cannon 1999

Hansford

1961

Hansford

1949

Meliola eveae F. Stevens Stevens 1928

1928

Meliola lictorea Cif. 1938 Ciferri 1938

Meliola feretiae Hansf.

1957

Meliola mitragynicola

Deighton 1958

Meliola thalliformis

Deighton 1958

Hansford

$1956 b$

Deighton

1957

Deighton

1957

Meliola vicina Syd. \& P. $\quad$ Sydow 1923a

Syd. 1923

Meliola galopinae Hansf. Hansford 1957

$1956 b$

Meliola microspora Pat.

\& Gaillard 1888

Meliola gonzalaguniae

(Cif.) Cif. 1961

Meliola duggenae $\mathrm{F}$.

Stevens 1928

Meliola duggenae $\mathrm{F}$.

Stevens 1928

Meliola kibirae Hansf. \&

F. Stevens 1937

Patouillard \&

Gaillard 1888

Ciferri 1961

Stevens 1928

Stevens 1928

\#Meliola bayamonensis

var. guettardae Cif. 1954

Meliola brachypoda Syd.

1922

Meliola

kanniyakumariana Hosag. 1996

Meliola henryi Hosag.

1996

Meliola oldenlandiae

Hansf. \& F. Stevens 1937

Meliola marthomaensis

Jacob Thomas et al. 2013

Meliola kibirae Hansf. \&

F. Stevens 1937

Asteridiella glabra (Berk.

$\&$ M.A. Curtis) Hansf.

1961

Meliola ixorae-coccineae

Hosag. \& C.M. Pillai

1994

Meliola thwaitesiana

Hansf. 1957
Hansford

1937

Ciferri 1954

Sydow 1922a

Hosagoudar

1996

Hosagoudar

1996

Hansford

1937

Thomas et al.

2013

Hansford

1937

Hansford

1961

Hosagoudar et al. 1994 b

Hansford $1956 \mathrm{~b}$ 
Ixora elongata

Ixora elongata

Ixora parviflora

Ixora philippinensis

Ixora sp.

Kadua affinis (Gouldia coriacea)

Kadua affinis (Gouldia coriacea)

Kadua affinis (Gouldia lanceolata/terminalis)

Kadua knudsenii

Knoxia sp.

Knoxia sp.

Knoxia sumatrensis (Knoxia corymbosa)

Lasianthus chinensis

(Mephitidia chinensis)

Lasianthus lancifolius

Lasianthus microphyllus

Leptactinia platyphylla*

Luculia grandifolia

Malanea glabra (Malanea macrophylla)

Mitchella repens

Mitragyna diversifolia

Mitragyna parvifolia

Mitragyna tubulosa

Morelia senegalensis

Morinda bartlingii

Morinda lucida

Mussaenda arcuata

Mussaenda frondosa
Meliola ixorae H.S. Yates Yates 1917 1917

Meliola ravii Hosag. et al. Biju et al. 2005 2005

Meliola chandolensis

C.R. Patil ex Hosag. 1996

Hosagoudar

Meliola ixorae H.S. Yates 1917

Asteridiella ixorae Hosag. Hosagoudar \& Archana 2010

\& Archana

2010

Meliola gouldiae Hansf. Hansford 1958

$1957 \mathrm{c}$

Meliola sandwicensis $\quad$ Ellis \&

Ellis \& Everh. 1895

Everhart 1895

Setameliola kaduae (F. Reynolds

Stevens) D.R. Reynolds 2010

2010

Meliola kauaiensis F.

Stevens 1925

Meliola

kanniyakumariana Hosag.

1996

Meliola knoxiicola Hosag. Biju et al. et al. 2005

2005

Meliola knoxiae Petr. Petrak 1958

1959

Meliola mephitidiae W. Index

Yamam. 1941

Fungorum

2016

!Meliola lasianthi Jana et Jana et al.

al. 2005

2005c

Meliola lasianthi Petr.

Petrak 1958

1959

Meliola leptactiniae

Mibey 1997

Mibey \&

Hawksworth 1997

Meliola luculiae Hosag. et Nithyatharani al. 2011

et al. 2011

Meliola malaneae $\mathrm{F}$.

Stevens \&

Stevens \& Tehon 1926

Tehon 1926

Meliola mitchellae Cooke Cooke 1878

1878

Meliola mitragynae Syd.

\& P. Syd. 1913

Sydow \&

Sydow 1914b

Meliola parvifoliae Suj.

Singh \& Kamal 1982

Meliola mitragynae-

tubulosae Hosag. \&

Manojk. 2005

Kamal \&

Singh 1982

Hosagoudar

2005

Meliola psychotriae Earle Earle 1904 1905

Meliola palawanensis

Syd. \& P. Syd. 1912

Sydow \&

Sydow 1912b

Meliola psychotriae Earle 1905

Meliola mussaendae- Hansford arcuatae Hansf. 1958

$1957 \mathrm{c}$

Meliola kibirae Hansf. \& Hansford

F. Stevens 1937

1937 
Mussaenda philippica

Mycetia javanica

Nauclea diderrichii

Neanotis monosperma

Neolamarckia cadamba

Neonauclea excelsa (Nauclea excelsa)

Neonauclea $\mathrm{sp}$.

Neonauclea sp.

Neurocalyx calycinus

Oldenlandia sp.

Oxyanthus sp.

Oxyceros patulus (Randia patula)

Palicourea croceoides (Palicourea riparia)

Palicourea domingensis

Palicourea domingensis

Palicourea domingensis

Palicourea sp.

Pavetta corymbosa (Pavetta baconia)

Pavetta sp.

Plectronia ventosa*

Polysphaeria parvifolia

Psychotria berteroana

Psychotria berteroana

Psychotria globicephala

Psychotria grandis
Meliola mussaendae Syd. Sydow \& \& P. Syd. 1917 Sydow 1917a Meliola mycetiae F. Stevens ex Deighton 1968 Meliola thalliformis Deighton 1958 Deighton 1967 Deighton 1957

Meliola neanotidis Hosag. Biju et al. et al. $2005 \quad 2005$

Meliola cadambae Hosag. Hosagoudar et \& C.K. Biju 2003

al. $2003 \mathrm{~b}$

Asteridiella naucleae

(Boedijn) Hansf. 1961

Hansford

1961

\#Irenina angustispora

var. laevis F. Stevens \&

Roldan 1935

Asteridiella angustispora

Ciferri 1954

F. Stevens \& Roldan ex

Hansford

Hansf. 1963

Meliola neurocalycis Jana Jana et al. et al. 2005

Meliola oldenlandiicola

Mibey 1997

2005d

Hansford

1954

Meliola psychotriae Earle Earle 1904

1905

Meliola randiicola Hansf. Hansford

1954

1954

Meliola mayaguesiana F. Stevens 1916

Stevens 1916

Asteridiella glabra (Berk. Hansford

\& M.A. Curtis) Hansf. 1961

1961

Asteridiella seminata

(Berk. \& M.A. Curtis)

Hansford

1961

Hansf. 1961

Meliola mayaguesiana F. Stevens 1916

Stevens 1916

Asteridiella palicoureae Hansford

Hansf. 1957

$1957 b$

Meliola psychotriae Earle Earle 1904

1905

Meliola psychotriae Earle Earle 1904 1905

Meliola falcata Syd. \& P. Sydow \& Syd. 1912

Cryptomeliola moiana

Sydow 1912a

Mibey \& Mibey 1997

Hawksworth 1997

Asteridiella glabra (Berk. Hansford \& M.A. Curtis) Hansf. 1961

1961

Asteridiella seminata Hansford

(Berk. \& M.A. Curtis)

1961

Hansf. 1961

Amazonia goosii Hosag.

\& T.K. Abraham 1998

Hosagoudar

\& Abraham

$1998 \mathrm{a}$

Asteridiella glabra (Berk. Hansford

\& M.A. Curtis) Hansf.

1961 
Psychotria kaduana

(Straussia kaduana)

Psychotria macrocarpa

Psychotria mariniana

(Straussia mariniana)

Psychotria mariniana

(Straussia mariniana)

Psychotria nigropunctata

Psychotria nudiflora

Psychotria pubescens

Psychotria pubescens

Psychotria pubescens

Psychotria recurva

Psychotria revoluta

Psychotria sp.

Psychotria sp.

Psychotria sp.

Psychotria sp.

Psychotria sp.

Psychotria sp.

Psychotria sp.

Psychotria sp.

Psychotria sp.

Psychotria sp.

Psychotria sp. (Straussia sp.)

Psychotria viridiflora

Psydrax dicoccos (Canthium dicoccum)

Psydrax odorata (Plectronia umbellata)

Psydrax parviflora (Canthium vulgare)
Setameliola kaduae (F.

Stevens) D.R. Reynolds

2010

Meliola ixorae H.S. Yates Yates 1917

1917

Amazonia polypoda Syd.

\& P. Syd. 1917

Sydow \&

Amazonia psychotriae

(Henn.) Theiss. 1913

Meliola psychotriae Earle 1905

Meliola psychotriaenudiflorae Hosag. 1996

!Meliola bayamonensis

var. bayamonensis Tehon

1919

Asteridiella glabra (Berk. Hansford

\& M.A. Curtis) Hansf.

1961

Setameliola

bayamonensis (Tehon)

D.R. Reynolds 2010

Meliola fasciculiseta Cif. Ciferri 1938

1938

Meliola mayaguesiana F. Stevens 1916

Stevens 1916

Amazonia asterinoides

(G. Winter) Theiss. 1913

Asteridiella

penicilliformis (Gaillard)

Hansf. 1961

Asteridiella vegabajensis

Hansf. 1957

Sydow $1917 b$

Theissen 1913

Earle 1904

Hosagoudar

1996

Tehon 1919

1961

Reynolds

2010

Meliola africana Hansf.

1957

Meliola hydei Hosag. et

al. 2005

Meliola longiseta Höhn.

1907

Meliola makilingiana

Syd. \& P. Syd. 1917

Meliola malaneae $\mathrm{F}$.

Stevens \& Tehon 1926

Meliola petiolariicola

Mibey 1997

Meliola rechingeri Hansf. 1958

Theissen 1913

Hansford

1961

Hansford

$1957 b$

Hansford

$1956 b$

Biju et al.

2005

Höhnel 1907

Sydow \&

Sydow 1917a

Stevens \&

Tehon 1926

Mibey \&

Hawksworth

1997

Hansford

$1957 \mathrm{c}$

Meliola straussiae Hansf.

1957

Hansford

$1957 b$

Amazonia psychotriae

Theissen 1913

(Henn.) Theiss. 1913

Meliola coilicosa L.N.

Nair \& V.P. Kaul 1983

Nair \& Kaul

1983

Meliola plectroniae

Hansford

1955

Reynolds

Setameliola canthii

(Hansf.) D.R. Reynolds

2010 
Randia aculeata

Randia sezitat*

Rhodopentas bussei (Pentas bussei)

Rondeletia purdiei

Rondeletia purdiei

Rondeletia sp.

Rothmannia hispida (Randia hispida)

Rothmannia longiflora

(Randia maculata)

Sabicea villosa (Sabicea

hirsuta)

Sarcocephalus latifolius

(Sarcocephalus esculentus)

Sherbournia bignoniiflora (Amaralia heinsioides)

Stenostomum lucidum

(Antirhea lucida)

Suberanthus brachycarpus

(Rondeletia brachycarpa)

Tamilnadia uliginosa

(Xeromphis uliginosa)

Tarenna asiatica (Webera corymbosa)

Timonius ternifolius

Tricalysia okelensis

(Tricalysia pobeguinii)

Uncaria africana

Uncaria elliptica (Uncaria

dasyoneura)

Uncaria guianensis

Uncaria longiflora

Uncaria perrottetii

Uncaria perrottetii

Uncaria sp.

Uncaria $\mathrm{sp.}$

Uncaria tomentosa

(Ourouparia tomentosa)

Undertermined
Meliola randiae-

aculeatae Hansf. 1955

Meliola dognyensis B.

Huguenin 1969

Hansford

1955

Index

Fungorum

2016

Meliola pentadis Mibey 1997

Mibey \&

Hawksworth

1997

Meliola amphigena $\mathrm{F}$.

Stevens \&

Stevens \& Tehon 1926

Tehon 1926

Meliola psychotriae Earle Earle 1904

1905

Asteridiella rondeletiifolii Batista et al.

Bat. \& Nascim. $1960 \quad 1960$

Meliola kibirae Hansf. \& Hansford

F. Stevens 1937

1937

Meliola kibirae Hansf. \&

F. Stevens 1937

Hansford

1937

Meliola sabiceae Cif.

1954

Asteridiella sarcocephali

(Hansf. \& Deighton)

Ciferri 1954

Hansf. 1961

Meliola amaraliae Hansf. Hansford \&

\& Deighton 1948 Deighton

1948

Meliola delae Schmied.

1989

Meliola isochaeta Cif.

1938

Irenopsis xeromphidis

Hosag. \& Sabeena 2010

Schmiedeknec

ht 1989

Ciferri 1938

Hosagoudar

\& Sabeena

2010

Meliola weberae J.N. Index

Kapoor 1967

Fungorum

2016

Meliola vicina Syd. \& P. $\quad$ Sydow 1923a

Syd. 1923

Meliola tricalysiae

Deighton 1958

Meliola deformis

Deighton 1958

Meliola uncariicola

Deighton 1958

Deighton

1957

Deighton

1957

Deighton

1958

Meliola anceps Syd. \& P. Sydow \&

Syd. 1916

Meliola cyrtochaeta Syd. 1928

Sydow 1916a

Hansford

$1957 \mathrm{c}$

Asteridiella uncariae

Hansford

1961

(Rehm) Hansf. 1961

Asteridiella uncariicola

Hansf. 1958

Asteridiella amoena

(Syd.) Hansf. 1961

Hansford

$1957 \mathrm{c}$

Hansford

1961

Asteridiella manca (Ellis Hansford

\& G. Martin) Hansf. 19611961

Meliola ouroupariae F. Stevens 1928

Stevens 1928

Asteridiella plebeja

(Speg.) Hansf. 1961
Hansford

1961 
Vahlia digyna (Oldenlandia decumbens)

Vangueria apiculata

Vangueria apiculata

Virectaria multiflora (Virecta multiflora)

Warszewiczia coccinea

Wendlandia scabra

Wendlandia thyrsoidea (Wendlandia notoniana) Wendlandia tinctoria

Rutaceae

Acronychia laevis

Acronychia pedunculata

Acronychia pedunculata

Acronychia pedunculata

Agathosma ovata (Barosma scoparia)

Amyris diatrypa

Amyris diatrypa

Amyris elemifera

Atalantia buxifolia

Atalantia monophylla

Atalantia sp.

Atalantia wightii

Balfourodendron riedelianum

Balfourodendron sp.

Barosma sp.

Bosistoa euodiformis

Bouchardatia neurococca

Casimiroa tetrameria

Citrus aurantiifolia (Citrus medica var. acida)
Meliola oldenlandiae

Hansford

Hansf. \& F. Stevens 19371937

Meliola mussaendae- Hansford arcuatae Hansf. $1958 \quad$ 1957c

Meliola vicina Syd. \& P. Sydow 1923a Syd. 1923

Meliola vicina Syd. \& P. Sydow 1923a Syd. 1923

Meliola amphigena $\mathrm{F}$.

Stevens \& Tehon 1926

Appendiculella

Stevens \&

Tehon 1926

Jana et al.

wendlandiae Jana et al. 2005a 2005

Meliola wendlandiae

Hosag. 1990

Meliola mitragynicola

Deighton 1958

Hosagoudar

\& Goos 1990a

Deighton

1957

Irenopsis moelleriana (G. Stevens 1927

Winter) F. Stevens 1927

Meliola koniaensis Hansf. Hansford \&

\& Deighton 1948

Deighton

1948

Meliola kisubiensis

Hansf. 1947

Hansford

$1947 \mathrm{a}$

Amazonia acronychiae

Hosag. 1989

Hosagoudar

\& Goos 1989

Asteridiella acronychia

Hu et al. 1987

Y.X. Hu 1987

Asteridiella acronychiae- Hosagoudar pedunculatae Hosag. 1991

Meliola microthecia

\& Goos 1991

Thümen 1876

Thüm. 1876

Meliola amyridis (Hansf.) Hansford

Minter 2006

1955

Meliola connaricola Hansford

Hansf. 19551955

Meliola monensis F. $\quad$ Stevens 1916

Stevens 1916

Meliola atalantiae (Pat.) Yamamoto

W. Yamam. $1958 \quad 1958$

Meliola atalantiae (Pat.) Yamamoto

W. Yamam. $1958 \quad 1958$

Meliola furcata Lév. 1846 Léveillé 1846

Meliola cranei Hosag. \& Hosagoudar et

Goos 1998

al. $1998 b$

Asteridiella obesa (Speg.) Hansford

Hansf. 1961

1961

Asteridiella obesa (Speg.) Hansford

Hansf. 1961

1961

Meliola thuemeniana F. Stevens 1928

Stevens 1928

Meliola kisubiensis Hansford

Hansf. 1947

Meliola bouchardatiae

Hansf. 1953

1947 a

Hansford

$1953 \mathrm{a}$

Asteridiella casimiroae

Hansford

Hansf. 1958

$1957 \mathrm{c}$

Amazonia butleri (Syd. \& Stevens 1927

P. Syd.) F. Stevens 1927 
Citrus maxima

Citrus maxima (Citrus decumana)

Citrus sp.

Citrus sp.

Citrus sp.

Clausena excavata

Clausena indica

Clausena sp.

Comptonella drupacea

Comptonella oreophila

(Evodia oreophila)

Eriostemon australasius

(Eriostemon lanceolatus)

Ertela trifolia (Monnieria

trifolia)

Evodia glabra*

Evodia sp.

Fagara chiloperone

Flindersia collina

Galipea longiflora

Glycosmis cochinchinensis

Glycosmis pentaphylla

Harrisonia abyssinica

Helietta apiculata (Helietta cuspidata)

Leionema dentatum

(Phebalium dentatum)

Luvunga eleutherandra*

Luvunga sp.

Medicosma cunnighamii

Melicope lunu-ankenda

(Euodia lunu-ankenda)

Melicope lunu-ankenda

(Euodia lunu-ankenda)
Meliola citri-maximae

Hyde et al.

X.Y. Zeng et al. 2016

2016

Amazonia butleri (Syd. \& Stevens 1927

P. Syd.) F. Stevens 1927

Meliola amyridis (Hansf.) Sydow \&

Minter 2006

Sydow 1917a

Meliola camelliae (Catt.) Saccardo

Sacc. 1882

1882

Meliola citricola Syd. \& $\quad$ Sydow \&

P. Syd. 1917

Sydow 1917a

Asteridiella obesa (Speg.) Hansford

Hansf. 1961

1961

Meliola clausenae Hosag. Hosagoudar

1990

Meliola clausenigena

Hosag. \& Riju 2011

\& Goos 1990a

Hosagoudar

Meliola comptonellae B.

Huguenin 1969

\& Riju 2011b

Index

Fungorum

2016

Meliola mouensis B. Index

Huguenin 1969

Fungorum

2016

Meliola baileyi Hansf. Hansford

1953

$1953 \mathrm{a}$

Meliola monnieriae F. Stevens 1928

Stevens 1928

Meliola euodiicola Hansf. Hansford

1954

1954

Meliola euodiae Pat. 1888 Doassans \&

Patouillard

1888

\#Meliola fagaricola Speg. Hansford

1924

$1956 b$

Meliola baileyi Hansf.

1953

Hansford

1953a

Meliola galipeae Syd. \&

P. Syd. 1916

Sydow \&

Yates 1917

Meliola glycosmidis J.N. Index

Kapoor 1967

Meliola harrisoniae

Hansf. 1938

Yates 1917

Fungorum

2016

Hansford

1938

Asteridiella obesa (Speg.) Hansford

Hansf. 1961

Meliola kisubiensis

Hansf. 1947

1961

Hansford

$1947 \mathrm{a}$

Meliola luvungae Hosag. Hosagoudar

1990

\& Goos 1990a

Meliola luvungicola

Hosag. \& G. Rajkumar 2007

Meliola kisubiensis

Hosagoudar et al. $2007 \mathrm{~b}$

Hansford $1947 \mathrm{a}$

Hansf. 1947

Hosagoudar

Amazonia melicopicola

Hosag. \& T.K. Abraham 1998

\& Abraham

$1998 \mathrm{a}$

Meliola melicopegena Hosagoudar

Hosag. \& S. Thomas 2013 \& Goos 1990a 
Melicope triphylla

Micromelum minutum*

Micromelum sp.

Murraya koenigii

Murraya sp.

Murraya sp.

Paramignya armata*

Pelea hawaiensis

Pelea sp.

Pelea sp.

Pelea sp.

Phebalium squamulosum*

Pilocarpus racemosus

Ptaeroxylon obliquum

Toddalia asiatica

Toddalia asiatica

Toddalia asiatica

Toddalia asiatica

Toddalia asiatica

Toddalia asiatica

Toddalia asiatica

Toddalia sp.

Toddalia sp.

Vepris bilocularis*

Vepris nobilis (Teclea nobilis)

Vepris undulata (Toddalia lanceolata)

Zanthoxylum elephantiasis

Zanthoxylum hyemale
Meliola melicopes Petr. 1959

!Meliola micromeli $\mathrm{F}$. Stevens \& Stevens \& Roldan $1935 \quad$ Roldan 1935 !Meliola micromeli T.K. Jana \& Ghosh Jana \& S.N. Ghosh 2010 Irenopsis murrayae Hosag. \& G. Rajkumar 2001

Meliola atalantiae (Pat.) W. Yamam. 1958 2010 Hosagoudar et al. 2001a

Meliola tenella Pat. 1888

Meliola paramignyae

Hosag. 1988

Meliola recurvipoda

Hansf. 1957

Meliola juddiana $\mathrm{F}$.

Stevens 1925

Meliola kisubiensis

Hansf. 1947

Yamamoto

1958

Doassans \&

Patouillard

1888

Hosagoudar

$1988 \mathrm{c}$

Hansford

$1957 b$

Stevens 1925

Hansford $1947 \mathrm{a}$

Meliola peleae F. Stevens Stevens 1925 1925

Meliola baileyi Hansf. Hansford 19531953 a

Meliola pilocarpi $\mathrm{F}$. Stevens 1916

Stevens 1916

Meliola ptaeroxyli Doidge Doidge 1941 1941

!Meliola toddaliae Hansf. Hansford 1937

1937

Asteridiella toddaliae

Hosag. \& Riju 2011

Meliola cannonicola

Hosag. \& C.K. Biju 2004

Meliola dasiana Mibey 1997

Hosagoudar \& Riju 2011b

Hosagoudar 2004b

Mibey \&

Hawksworth

1997

Meliola kisubiensis Hansford

Hansf. 1947 1947a

Meliola tecleae Hansf. Hansford 1941

1941

Meliola toddaliicola

Hansford Hansf. $1947 \quad 1947 \mathrm{a}$

Meliola cadigensis H.S. Yates 1917

Yates 1917

Meliola devikulamensis Hosagoudar

Hosag. et al. $2005 \quad 2005$

Meliola vepridis Hosag. et Hosagoudar et al. 1994

Meliola tecleae Hansf. 1941

al. 1994 a

Hansford

1941

Meliola toddaliae Doidge Doidge 1917

1917

Asteridiella trachylaena

(Syd.) Hansf. 1961

Hansford

1961

!Asteridiella fagaricola var. zanthoxyli Hansf.

1957
Hansford

$1956 b$ 
Zanthoxylum juniperinum

(Zanthoxylum procerum)

Zanthoxylum juniperinum

(Zanthoxylum procerum)

Zanthoxylum laurentii

(Fagara laurentii)

Zanthoxylum leprieurii

(Fagara angolensis)

Zanthoxylum limoncello

Zanthoxylum linealis*

(Fagara linealis)

Zanthoxylum martinicense

Zanthoxylum martinicense

(Fagara martinicensis)

Zanthoxylum nitidum

Zanthoxylum nitidum (Fagara nitida)

Zanthoxylum ovalifolium

Zanthoxylum ovata*

Zanthoxylum rhetsa*

Zanthoxylum sp.

Zanthoxylum sp.

Zanthoxylum sp.

Zanthoxylum tetraspermum*

Sabiaceae

Salicaceae

Meliosma rigida

Meliosma simplicifolia

Meliosma sp.

Meliosma sp.

Banara guianensis

Casearia aculeata

Casearia aculeata (Samyda

pubescens)

Casearia arborea

Casearia arborea

Casearia arborea

Casearia battiscombei

Casearia bicolor*
Meliola aterrima Syd.

Sydow 1926

1926

Meliola macropoda Syd. Sydow 1926

1926

Meliola yangambiensis Hansford

Hansf. 1947 1947b

Meliola fagarae Hansf. Hansford

1941

1941

Asteridiella trachylaena Hansford

(Syd.) Hansf. 1961

\#Meliola fagaricola Speg. Spegazzini

1924

\#Meliola fagaricola Speg. Farr \&

1924

Farr \&

Rossman

2016

Meliola fagarae-

Hansford

martinicensis Hansf. 19491949

\#Meliola fagaricola Speg. Farr \& 1924

Rossman

2016

Hansford

1961

Hansf. 1961

Hosagoudar

1996

ovalifolii Hosag. 1996

Meliola rickiana Hansf.

1955

Hansford

1955

!Meliola vatsavayae

Hosagoudar

Hosag. \& Riju 2009

\& Riju 2009

Asteridiella obesa (Speg.) Hansford

Hansf. 1961

1961

Asteridiella tomentosa (G. Hansford

Winter) Hansf. 1961

1961

Meliola edanoana $\mathrm{F}$.

Hansford

Stevens ex Hansf. 1957

$1957 b$

Meliola zanthoxyli Hansf. Hansford

1947

1947 a

Meliola hiratsukana

Katumoto

1991

Katum. 1991
Asteridiella meliosmae

A.K. Kar \& Maity 1971

Kar \& Maity

1970 a

!Asteridiella meliosmicola $\mathrm{Hu} 1990 \mathrm{~b}$

$\mathrm{H}$. $\mathrm{Hu} 1990$

Meliola meliosmae Petr.

1959

Meliola banarae F.

Stevens 1928

Meliola banarae $\mathrm{F}$.

Stevens 1928

Meliola samydae Cif.

1931

!Meliola melanochaeta Sydow 1928

Cif. 1954

Irenopsis casearina

Hansf. 1957

Hansford

$1957 b$

Hansford

$1956 b$

arboreae Hansf. 1957

Mibey et al.

1996

Mibey 1996

Ciferri 1954

Meliola hypodoria Cif. 1954 
Casearia engleri

Casearia engleri

Casearia esculenta

Casearia guianensis

Casearia guianensis

Casearia guianensis

Casearia guianensis

Casearia ilicifolia

Casearia sylvestris

Casearia villilimba

Dovyalis macrocalyx

Dovyalis rhamnoides

Dovyalis zeyheri (Dovyalis tristis)

Flacourtia sp.

Flacourtia sp.

Hasseltia floribunda

Homalium angustifolium*

Homalium barandae*

Homalium lalaense\#

Homalium le-testui

(Homalium dolichophyllum)

Homalium le-testui

(Homalium dolichophyllum)

Homalium zeylanicum*

Oncoba sp.

Oncoba sp.

Oncoba spinosa

Scolopia crassipes*

Scolopia crenata*

Scolopia crenata*
Asteridiella caseariae

(Hansf.) Hansf. 1961

Meliola caseariicola

Hansf. 1947

Asteridiella caseariicola

Hosag. 1996

Meliola ambigua Pat. \&

Gaillard 1888

Meliola caseariae-

arboreae Hansf. 1957

Meliola caseariae-

guianensis Hansf. 1955

Meliola kernii Hansf.

1963

Meliola trichocarpa Cif.

1938

Meliola caseariae Petr. \&

Cif. 1930

!Appendiculella

caseariicola $\mathrm{H}$. Hu 1990

Appendiculella natalensis

(Doidge) Hansf. 1961

Appendiculella natalensis

(Doidge) Hansf. 1961

Appendiculella natalensis

(Doidge) Hansf. 1961

Amazonia flacourtiae

Hosag. et al. 1993

Meliola arippaensis

Hosag. \& A. Sabeena

2013

Meliola convallata Petr.

1937

Asteridiella homalii-

angustifolii (Deighton)

Hansf. 1961

Meliola batangasensis

Hansf. 1957

Meliola homalii Hansf.

1945

Meliola homaliicola

Deighton 1958

Meliola homalii-

dolichophylli Hansf. \&

Deighton 1948

Asteridiella homaliigena

Hosag. et al. 2011

Irenopsis claviculata

(Doidge) F. Stevens 1927

Irenopsis oncobae

(Henn.) Hansf. 1961

Appendiculella natalensis

(Doidge) Hansf. 1961

Meliola evansii Doidge

1920

Asteridiella scolopiae

Hosag. 1996

Meliola evansii Doidge

1920
Hansford

1961

Hansford

1947b

Hosagoudar

1996

Patouillard \&

Gaillard 1888

Hansford

$1956 b$

Hansford

1955

Hansford

1962

Ciferri 1938

Petrak \&

Ciferri 1930

$\mathrm{Hu}$ 1990b

Hansford

1961

Hansford

1961

Hansford

1961

Index

Fungorum

2016

Hosagoudar

2013a

Sydow \&

Petrak 1937

Hansford

1961

Hansford

1957b

Hansford

$1945 b$

Deighton

1957

Hansford \&

Deighton

1948

Hosagoudar et

al. $2011 \mathrm{~b}$

Stevens 1927

Hansford

1961

Hansford

1961

Doidge 1920b

Hosagoudar

1996

Doidge 1920b 
Scolopia rhaniphylla

Scolopia sp.

Scolopia sp.

Scolopia zeyheri

Xylosma buxifolia*

Xylosma buxifolia*

Xylosma intermedia

(Myroxylon intermedium)

Xylosma prockia (Xylosma salzmannii)

Xylosma sp.

Xylosma spiculifera

Salvadoraceae

Santalaceae
Azima tetracantha

Dendrophthora serpyllifolia (Dendrophthora biseriata) Dendrotrophe varians (Dendrotrophe frutescens) Exocarpos latifolius

Iodina rhombifolia*

Jodina sp.

Osyris compressa*

Osyris lanceolata (Osyris abyssinica)

Osyris lanceolata (Osyris abyssinica)

Osyris lanceolata (Osyris arborea)

Osyris lanceolata (Osyris arborea)

Osyris lanceolata (Osyris arborea)

Osyris lanceolata (Osyris quadripartita)

Scleropyrum pentandrum

Scleropyrum pentandrum

Scleropyrum pentandrum (Scleropyrum wallichianum) Viscum articulatum

Viscum sp.
Meliola flacourtiacearum Hansf. 1947

!Asteridiella scolopiae var. indica Hosag. \& Riju 2011

Meliola scolopoliicola $\mathrm{H}$.

Biju \& Bagool 2004

Meliola scolopiae Doidge 1928

Meliola xylosmaebuxifoliae Deighton 1968

Meliola xylosmicola

Hansf. 1955

Meliola xylosmae F.

Stevens 1928

Meliola tonduzii Speg.

191

Meliola alternipes (Speg.)

Hansf. 1955

Meliola xylosmicola

Garcés 1944

Meliola azimae Doidge

1928

Meliola epiviscum Cif. 1954

Meliola dendrotrophicola

H. Hu \& J.C. Yang 1989

Meliola exocarpi H.S.

Yates 1918

Asteridiella megalospora

(Speg.) Hansf. 1961

Asteridiella megalospora

(Speg.) Hansf. 1961

Meliola osyridis Doidge 1928

Meliola karamojensis

Hansf. 1947

Meliola polytricha

Kalchbr. \& Cooke 1879

Meliola mahamulkarii

Hosag. et al. 2010

Meliola osyridicola

Hansf. 1946

Meliola santalacearum

Budathoki \& P.N. Singh

1994

Meliola osyridicola

Hansf. 1946

!Meliola scleropyricola

Hosag. \& A. Sabeena

2013

Meliola scleropyri Hosag. 1990

Meliola hainanensis Y.X. Hu 1989

Meliola visci F. Stevens

1925

Meliola arcuata Doidge 1917
Hansford

1947b

Hosagoudar

\& Riju 2011a

Biju \&

Bagool 2004

Doidge \&

Sydow 1928

Deighton

1967

Hansford

1955

Stevens 1928

Spegazzini

1919a

Hansford

1955

Orejuela 1944

Doidge \&

Sydow 1928

Ciferri 1954

Hu \& Yang

1989

Yates 1918

Hansford

1961

Hansford

1961

Doidge \&

Sydow 1928

Hansford

$1947 \mathrm{a}$

Cooke 1879

Hosagoudar et al. $2010 \mathrm{~b}$

Hansford

1946

Budathoki \&

Singh 1994

Hansford

1946

Hosagoudar

\& Sabeena

2013c

Hosagoudar

\& Goos 1990a

$\mathrm{Hu} \& \mathrm{Lu}$

1989

Stevens 1925

Doidge 1917 
Meliola ecuadorensis F.

Stevens 1928

Meliola parenchymatica

Gaillard 1892

Meliola sapindacearum

Speg. 1891

Meliola stevensii Beeli

1920

Meliola terecitensis

Hansf. 1957

Meliola variaseta $\mathrm{F}$.

Stevens 1928

Acer negundo

Acer oblongum

Alectryon subcinereum

Allophylus africanus

Allophylus cobbe (Allophylus serrulatus)

Allophylus cobbe (Allophylus serrulatus)

Allophylus cominia

Allophylus concanicus (var. lanceolatus)

Allophylus crassinervis

Allophylus pervillei

Allophylus rubifolius

Allophylus sp.

Allophylus sp.

Allophylus sp.

Arytera sp.

Atalaya natalensis

Blighia sapida

Blighia unijugata

(Phialodiscus unijugatus)

Cardiospermum grandiflorum
Asteridiella negundinis

J.A. Stev. 1969

Meliola aceris W.

Yamam. 1940

Meliola alectryonis

Hansf. 1953

Meliola capensis

(Kalchbr. \& Cooke)

Theiss. 1912

Meliola allophyligena

Hosag. 2006

Meliola allophyli-

serrulati Hosag. \& T.K.

Abraham 1998

Meliola integriseta

(Speg.) Speg. 1924

Meliola allophyli-

Meliola capensis

(Kalchbr. \& Cooke)

Theiss. 1912

Meliola allophyli Doidge 1928

Meliola rubifolii Mibey 1997

Meliola allophylorum Mibey 1997

Meliola digoana Mibey 1997

Meliola furcillata Doidge 1917

Meliola colladoi Syd. \& P. Syd. 1920

Meliola atalayae Doidge 1948

Meliola capensis

(Kalchbr. \& Cooke)

Theiss. 1912

Meliola capensis

(Kalchbr. \& Cooke)

Theiss. 1912

Meliola mulleri Toro

1940 concanici Hosag. 1994
Stevens 1928

Gaillard 1892

Spegazzini

$1891 b$

Beeli 1920

Hansford

$1957 b$

Stevens 1928

Index

Fungorum

2016

Yamamoto

1940

Hansford

1953a

Theissen 1912

Hosagoudar 2006

Hosagoudar

\& Abraham

1998a

Spegazzini

1924

Hosagoudar et al. 1994b

Theissen 1912

Doidge \&

Sydow 1928

Mibey \&

Hawksworth

1997

Mibey \&

Hawksworth

1997

Mibey \&

Hawksworth

1997

Doidge 1917

Sydow \&

Sydow 1920b

Doidge 1948

Theissen 1912

Theissen 1912

Chardón et al.

1940 
Cardiospermum sp.

Cupania americana

Cupania americana

Cupania americana

Cupania emarginata

Cupania glabra

Cupania guatemalensis

Cupania guatemalensis

Cupania guatemalensis

Cupania sp.

Deinbollia borbonica

Deinbollia fulvotomentella

Deinbollia oblongifolia (Sapindus oblongifolius)

Dimocarpus longan

Dimocarpus longan

Dimocarpus longan

Dimocarpus longan

(Euphoria cinerea)

Dimocarpus longan (Nephelium longana)

Diploglottis cunninghamii (Diploglottis australis)

Dodonaea sp.

Dodonaea triquetra

Dodonaea viscosa

Elattostachys sp.

Erioglossum rubiginosum*
Meliola cardiospermi

Hansford

Hansf. \& F. Stevens 19371937

Asteridiella cupaniae Hansford

Hansf. 1963

1962

Asteridiella tersa (Cif.) Hansford

Hansf. 1961

1961

Irenopsis cupaniae (F.

Stevens 1927

Stevens) F. Stevens 1927

Asteridiella

Hansford guatemalensis (Hansf.) 1961

Hansf. 1961

Asteridiella guatemalensis (Hansf.)

Hansf. 1961

Appendiculella chiriquiensis Rodr. Just. \& M. Piepenbr. 2007

Asteridiella guatemalensis (Hansf.) Hansf. 1961

Irenopsis cupaniicola

Hansf. 1955

Hansford

1961

Rodríguez

Justavino \&

Piepenbring

2007

Hansford

1961

Hansford

1955

Asteridiella obesa (Speg.) Hansford

Hansf. 1961

Meliola borbonicae

1961

Mibey 1997

Mibey \&

Hawksworth

1997

Meliola deinbolliae Hansford

Hansf. 1937

1937

Meliola doidgeae Syd. $\quad$ Doidge \&

1928

Sydow 1928

Meliola capensis

(Kalchbr. \& Cooke)

Theiss. 1912

Meliola dimocarpi Hosag. Hosagoudar et \& T.K. Abraham 1998

Meliola thailandicum

al. $1998 \mathrm{e}$

Hongsanan et Hongsanan \& K.D. Hyde al. 2015

2015

Meliola capensis

Theissen 1912

(Kalchbr. \& Cooke)

Theiss. 1912

Irenopsis nephelii M.S.

Patil \& Maham. 1999

Meliola capensis

(Kalchbr. \& Cooke)

Theiss. 1912

Meliola aegiphilae F.

Stevens 1927

Asteridiella dodonaeae

(Hansf.) Hansf. 1961

Meliola lyoniae $\mathrm{F}$.

Stevens 1925

Meliola elattostachydis

Petr. 1959

Meliola erioglossi $\mathrm{F}$.

Stevens ex Hansf. 1957
Patil \&

Mahamulkar

1999

Theissen 1912

Hansford

1961

Hansford

1961

Stevens 1925

Petrak 1958

Hansford

$1957 b$ 
Exothea paniculata

Filicium decipiens

Filicium decipiens

Ganophyllum abliquum

Ganophyllum falcatum

Guioa microcarpa

Guioa semiglauca

Harpullia arborea

Harpullia arborea

Hippobromus pauciflorus

(Hippobromus alata)

Hypelate trifoliata

Koelreuteria sp.

Lecaniodiscus cupanioides

Lepisanthes senegalensis

Lepisanthes sp.

Lepisanthes umbellata\#

Litchi chinensis

Lychnodiscus dananensis

Matayba scrobiculata

Melicoccus bijugatus

(Melicocca bijuga)

Mischocarpus sp.

Mischocarpus sundaicus

Nephelium intermedium

Nephelium mutabile
Asteridiella stevensonii

(Cif.) Hansf. 1961

Meliola filicii Hosag.

1991

Meliola filiciicola Hosag. et al. 1996

Meliola ganophyni $\mathrm{F}$.

Stevens \& Roldan ex

Hansf. 1963

Meliola ganophyni $\mathrm{F}$.

Stevens \& Roldan ex

Hansf. 1963

Irenopsis araneosa (Syd.

\& P. Syd.) F. Stevens

1927

Meliola guioaesemiglaucae Hansf. 1953

Meliola harpullicola

Hosag. \& A. Sabeena

2013

Meliola odontocephala

Syd. 1925

Meliola capensis

(Kalchbr. \& Cooke)

Theiss. 1912

Asteridiella hypelates

Hansf. 1957

Meliola koelreuteriae

Hansf. 1948

Meliola capensis

(Kalchbr. \& Cooke)

Theiss. 1912

Meliola othophorae H.S.

Yates 1918

Meliola lepisanthea Sacc.

1917

Meliola lepianthis Hosag.

\& Kamar. 2001

Meliola capensis

(Kalchbr. \& Cooke)

Theiss. 1912

Meliola lychnodisci

Deighton 1958

Meliola mataybae F.

Stevens 1928

Meliola crucifera

Starbäck 1905

Meliola fraserae Hansf.

1953

Meliola capensis

(Kalchbr. \& Cooke)

Theiss. 1912

Meliola nepheliicola $\mathrm{F}$. Yamamoto

Stevens \& Roldan ex W. 1958

Yamam. 1958

!Meliola commixta Syd. Sydow 1925

1952 eighton

Hansford

1961

osagoudar

1991c

Hosagoudar

1996

Hansford

1962

Stevens \&

Roldan 1935

Stevens 1927

Hansford

1953a

Hosagoudar

2013a

Sydow 1925

Theissen 1912

Hansford

1957b

Hansford

1948

Theissen 1912

Yates 1918

Saccardo

1917

Theissen 1912

1957

Stevens 1928

Farr \&

Rossman

2016

Hansford

1953a

Theissen 1912 
Nephelium mutabile

Nephelium mutabile

Otonephelium stipulaceum

Otophora fruticosa*

Otophora sp.

Pancovia bijuga

Paullinia pinnata

Paullinia pinnata

Paullinia pinnata

Paullinia pinnata

Paullinia rubiginosa

Paullinia sp.

Paullinia sp.

Placodiscus riparius

Placodiscus splendidus

Sapindus emarginatus

Sapindus emarginatus

Sapindus saponaria

Sapindus saponaria

Sapindus sp.

Schleichera oleosa

Schmidelia sp.

Schmidelia sp.

Serjania diversifolia

Serjania glabrata (Serjania fulta)

Serjania paucidentata
Meliola capensis

Theissen 1912

(Kalchbr. \& Cooke)

Theiss. 1912

Meliola commixta Theiss. Theissen 1919 1919

Meliola otonephelii Hosagoudar

Hosag. 1990

\& Goos 1990a

Meliola othophorae H.S. Yates 1918

Yates 1918

!Meliola benguetensis F. Stevens \&

Stevens \& Roldan $1935 \quad$ Roldan 1935

Meliola capensis

(Kalchbr. \& Cooke)

Theiss. 1912

Meliola hessii F. Stevens

1916

Meliola paulliniae F.

Stevens 1916

Meliola paulliniifoliae

Bat. 1960

Meliola pinnatae Mibey 1997

Meliola paulliniifoliae

Bat. 1960

Theissen 1912

Stevens 1916

Stevens 1916

Batista et al.

1960

Mibey \&

Hawksworth

1997

Batista et al.

1960

Meliola paulliniana Bat.

\& Nascim. 1956

Batista et al.

1956

Meliola paulliniicola

Hansf. 1955

Hansford

1955

Meliola capensis

(Kalchbr. \& Cooke)

Theiss. 1912

Meliola capensis

Theissen 1912

(Kalchbr. \& Cooke)

Theiss. 1912

Appendiculella sapindi

T.P. Devi \& Choudhry 2005

Meliola capensis

(Kalchbr. \& Cooke)

Theiss. 1912

Meliola sapindi $\mathrm{F}$.

Stevens 1928

Meliola sydowiana $\mathrm{F}$.

Stevens \& Larson 1928

!Meliola anfracta Dilcher 1965

Meliola capensis

(Kalchbr. \& Cooke)

Theiss. 1912

Irenopsis laevis (Berk. \& Hansford

M.A. Curtis) Hansf. 1955

Meliola lepisanthea Sacc.

1917

1955

Saccardo

1917

Meliola serjaniae $\mathrm{F}$.

Stevens 1916

Stevens 1916

Meliola integriseta

(Speg.) Speg. 1924

Meliola serjaniicola $\mathrm{F}$.

Stevens \& Tehon 1926
Spegazzini

1924

Stevens \&

Tehon 1926 
Serjania polyphylla

Serjania polyphylla

Serjania sinuata*

Serjania sp.

Serjania sp.

Serjania sp.

Serjania triquetra

Talisia esculenta

Talisia esculenta (Sapindus esculentus)

Thouinia striata

Trigonachras membranacea*

Sapotaceae

Chrysophyllum albidum

Chrysophyllum sp.

Isonandra lanceolata

Madhuca neriifolia

Manilkara zapota (Lucuma mammosa)

Manilkara zapota (Lucuma mammosa)

Mimusops sp.

Palaquium sp.

Palaquium sp.

Palaquium sp.

Planchonella sandwicensis (Sideroxylon sandwicense)

Pouteria dictyoneura

(Paralabatia fuertesii)

Pouteria multiflora (Lucuma multiflora)

Pradosia lactescens

Sersalisia $\mathrm{sp}$.
Meliola acrotricha Syd. 1925

Meliola serjaniae F.

Stevens 1916

Meliola phaeocephala

Cif. 1954

!Meliola serjaniicola Bat. \& A.F. Vital 1953

Asteridiella chardoniana

Hansf. 1957

Meliola trujillensis Toro

1934

Meliola serjaniae F.

Stevens 1916

Meliola talisiana Bat. \&

H. Maia 1956

Meliola sapindi-esculenti

Hansf. 1955

Meliola thouiniae Earle

1905

Meliola acrotricha Syd.

1925

Asteridiella sapotacearum

(Hansf.) Hansf. 1961

Meliola cryptica $\mathrm{S}$.

Hughes 1953

Irenopsis chrysophylli

Rodr. Just. 2014

Meliola jayachandranii

Hosag. 1988

Meliola madhucae Hosag. et al. 2013

Asteridiella lucumae (Cif.

\& Gonz. Frag.) Hansf.

1961

Meliola domingensis Cif.

1954

Meliola snowdenii Hansf.

\& F. Stevens 1937

Amazonia palaquii

Hosag. \& P.J. Robin 2011

Meliola palaquii $\mathrm{F}$.

Stevens \& Roldan ex

Hansf. 1963

Meliola palaquiicola

Hansf. 1958

Meliola sideroxyli $\mathrm{F}$.

Stevens 1925

Meliola paralabatiae Cif. Ciferri 1933

1933

Meliola lucumae F.

Stevens 1916

Setameliola pradosiae

(Bat.) D.R. Reynolds

2010

Meliola sersalisiae Hansf.

1944
Sydow 1925

Stevens 1916

Ciferri 1954

Index

Fungorum

2016

Hansford

$1956 b$

Chardón \&

Toro 1934

Stevens 1916

Batista et al.

1956

Hansford

1955

Earle 1904

Sydow 1925

Hansford

1961

Hughes 1953

Rodríguez

Justavino et

al. 2015

Hosagoudar

$1987 \mathrm{a}$

Thimmaiah et

al. 2013

Hansford

1961

Ciferri 1954

Hansford

1937

Hosagoudar

\& Robin

2011a

Hansford

1962

Hansford

$1957 \mathrm{c}$

Stevens 1925

Stevens 1916

Reynolds

2010

Hansford

1944 
Sideroxylon foetidissimum

Sideroxylon foetidissimum (Mastichodendron foetidissimum)

Sideroxylon hirtiantherum

(Bumelia parviflora)

Sideroxylon salicifolium

(Dipholis salicifolia)

Sideroxylon $\mathrm{sp}$.

Synsepalum brevipes

(Pachystela brevipes)

Vitellaria paradoxa

(Butyrospermum parkii)

Saxifragaceae Astilbe philippinensis

Schisandraceae Illicium angustisepalum

Illicium chamaedrifoliae\#

Illicium parviflorum

Illicium parviflorum

Illicium $\mathrm{sp}$.

Kadsura coccinea

Kadsura japonica

Schlegeliaceae Schlegelia sp.

Schoepfia chinensis

Schoepfia obovata

Thomandersia laurifolia

Scrophulariaceae

\section{Buddleja americana \\ Buddleja americana \\ Buddleja asiatica \\ Buddleja auriculata}

Buddleja nitida

Buddleja parviflora (Buddleja intermedia)
Meliola sideroxylicola

J.A. Stev. 1969

Meliola sapotacearum

Mig. Rodr. \& Camino 1987

Meliola bumeliae Hansf. 1957

Meliola dipholidis $\mathrm{F}$.

Stevens 1916

Amazonia sideroxyli

Hansf. 1949

Meliola pachystelae

Mibey 1997

Meliola butyrospermi

Hansf. \& Deighton 1948

Asteridiella astilbincola

Petr. 1959

Appendiculella illicii B.

Song 1998

Meliola ilicis Henn. 1895

Asteridiella illicii Z.X.

Chen et al. 1993

Meliola illicii (Cif.)

Hansf. 1955

Meliola khasiensis

(Hansf.) Hansf. 1961

Asteridiella kadsuricola

H. Hu \& J.C. Yang 1989

Meliola kadsurae W.

Yamam. 1941

Asteridiella schlegeliae

(F. Stevens) Hansf. 1961

Meliola schoepfiae B.

Song \& Y.X. Hu 1997

Meliola ekmaniana Cif.

1954

Meliola thomandersiae

Hansf. 1958

Meliola ulei Hansf. 1949

Asteridiella buddlejae

(Hansf.) Hansf. 1961

Asteridiella buddlejicola

(Henn.) Hansf. 1961

Meliola pampangensis

Petr. 1959

!Meliola quinquespora

Thüm. 1876

Asteridiella nitidae Rodr. Just. 2014

Asteridiella obducens

(Gaillard) Hansf. 1961
Index

Fungorum

2016

Rodríguez \&

Camino 1987

Hansford

$1956 \mathrm{~b}$

Stevens 1916

Hansford

1949

Mibey \&

Hawksworth

1997

Hansford \&

Deighton

1948

Petrak 1958

Song 1998

Index

Fungorum

2016

$\mathrm{Hu}$ et al.

1993a

Hansford

1955

Hansford

1961

Hu \& Yang

1989

Yamamoto

$1941 b$

Hansford

1961

Song et al.

1997c

Ciferri 1954

Hansford

$1957 \mathrm{c}$

Hansford

1949

Hansford

1961

Hansford

1961

Petrak 1958

Index

Fungorum

2016

Rodríguez

Justavino et

al. 2015

Hansford

1961 


\begin{tabular}{|c|c|c|c|}
\hline & Buddleja saligna (Chilianthus & Asteridiella implicata & Hansford \\
\hline & & & \\
\hline & Buddleja sp. & Appendiculella sororcula & $\begin{array}{l}\text { Hansford } \\
1961\end{array}$ \\
\hline & Buddleja sp. & Asteridiella arachnoidea & Hansford \\
\hline \multirow{4}{*}{ Selaginellaceae } & Selaginella pilifera ( var. & Asteridiella selaginellae & Farr \& Horner \\
\hline & pringlei) & M.L. Farr 1968 & 1968 \\
\hline & Selaginella plumosa & Irenopsis selaginellarum & Hansford \\
\hline & (Selaginella stolonifera) & (Cif.) Hansf. 1961 & 1961 \\
\hline \multirow[t]{19}{*}{ Simaroubaceae } & Ailanthus triphysa & $\begin{array}{l}\text { Meliola ailanthi J.K. } \\
\text { Sharma et al. } 1985\end{array}$ & $\begin{array}{l}\text { Sharma et al. } \\
1985\end{array}$ \\
\hline & Ailanthus triphysa & Meliola ailanthicola & Hosagoudar \\
\hline & & Hosag. \& Riju 2010 & \& Riju 2010 \\
\hline & Castela coccinea & Meliola alternipes (Speg.) & Hansford \\
\hline & & Hansf. 1955 & 1955 \\
\hline & Castela tortuosa & Meliola castelae J.A. & Index \\
\hline & & Stev. 1969 & $\begin{array}{l}\text { Fungorum } \\
2016\end{array}$ \\
\hline & Castela tweediei & Meliola alternipes (Speg.) & Hansford \\
\hline & & Hansf. 1955 & 1955 \\
\hline & Picrasma javanica & Meliola picrasmae Hansf. & Hansford \\
\hline & Picrasma iavanica & $\begin{array}{l}1955 \\
\text { Meliola nicrasmae Hansf }\end{array}$ & $\begin{array}{l}1955 \\
\text { Hansford }\end{array}$ \\
\hline & Pıcrasma javanica & $\begin{array}{l}\text { Mellola picrasmae Hansi. } \\
1955\end{array}$ & 1955 \\
\hline & Quassia gabonensis (Hannoa & Meliola hannoae & Deighton \\
\hline & klaineana) & Deighton 1958 & 1957 \\
\hline & Quassia indica (Samadera & Meliola samaderae & Hosagoudar et \\
\hline & indica) & Hosag. et al. 2003 & al. $2003 \mathrm{a}$ \\
\hline & Simarouba sp. & Asteridiella simarubicola & Hansford \\
\hline & & $\begin{array}{l}\text { Hansf. } 1958 \\
\text { Asteridiella simarubae }\end{array}$ & $\begin{array}{l}1957 \mathrm{c} \\
\text { Hancford }\end{array}$ \\
\hline & simarouba tulae & $\begin{array}{l}\text { Asteridiella simarubae } \\
\text { Hansf. } 1957\end{array}$ & $\begin{array}{l}\text { Hanstord } \\
1957 b\end{array}$ \\
\hline \multirow[t]{2}{*}{ Siparunaceae } & Siparuna gesnerioides & Meliola siparunae Syd. & Sydow \& \\
\hline & (Siparuna patelliformis) & 1929 & Petrak 1929 \\
\hline \multirow[t]{8}{*}{ Smilacaceae } & Smilax coriacea & $\begin{array}{l}\text { Meliola smilacis } \mathrm{F} . \\
\text { Stevens } 1916\end{array}$ & Stevens 1916 \\
\hline & Smilax populnea & $\begin{array}{l}\text { Meliola gregoriana } \mathrm{F} . \\
\text { Stevens } 1925\end{array}$ & Stevens 1925 \\
\hline & Smilax sp. & Meliola loganiensis Sacc. & Index \\
\hline & & \& Berl. 1885 & $\begin{array}{l}\text { Fungorum } \\
2016\end{array}$ \\
\hline & Smilax sp. & Meliola smilacacearum & Hosagoudar \\
\hline & & Hosag. 2006 & 2006 \\
\hline & Smilax zeylanica & $\begin{array}{l}\text { Meliola gamblei Hosag. } \\
1991\end{array}$ & $\begin{array}{l}\text { Hosagoudar } \\
\& \text { Goos } 1991\end{array}$ \\
\hline & Smilax zeylanica & Meliola salleana Hansf. & Hansford \\
\hline \multirow{9}{*}{ Solanaceae } & Acnistus arborescens & Asteridiella portoricensis & $\begin{array}{l}1902 \\
\text { Hansford }\end{array}$ \\
\hline & & (Toro) Hansf. 1961 & 1961 \\
\hline & Acnistus arborescens & $\begin{array}{l}\text { Meliola borinquena Cif. } \\
1954\end{array}$ & Ciferri 1954 \\
\hline & Acnistus sp. & $\begin{array}{l}\text { Asteridiella portoricensis } \\
\text { (Toro) Hansf. } 1961\end{array}$ & $\begin{array}{l}\text { Hansford } \\
1961\end{array}$ \\
\hline & Brunfelsia americana & Asteridiella plebeja & Hansford \\
\hline & (Brunfelsia abbottii) & (Speg.) Hansf. 1961 & 1961 \\
\hline & Capsicum baccatum & $\begin{array}{l}\text { Meliola capsicola } \mathrm{F} \text {. } \\
\text { Stevens } 1916\end{array}$ & Stevens 1916 \\
\hline & Cestrum latifolium & Asteridiella tabaquitensis & Hansford \\
\hline & & Hansf. 1957 & $1956 b$ \\
\hline
\end{tabular}


Cestrum laurifolium

Cestrum macrophyllum

Cestrum macrophyllum

Cestrum schlechtendahlii

(Cestrum megalophyllum)

Cestrum sp.

Cestrum sp.

Cestrum sp.

Discopodium penninervium

Duboisia myoporoides

Physalis peruviana

Physalis sp.

Schwenckia lateriflora

(Schwenckia browallioides)

Schwenckia lateriflora

(Schwenckia browallioides)

Solanum amygdalifolium

(Solanum persicifolium)

Solanum boerhaaviifolium

Solanum campaniforme

Solanum englerianum*

Solanum erythrotrichum

Solanum giganteum

Solanum jamaicense

Solanum nudum (Solanum antillarum)

Solanum rugosum

Solanum sp.

Solanum sp.

Solanum sp.

Solanum sp.

Solanum sp.

Solanum sp.
Meliola gesneriae F.

Stevens 1916

Meliola cestri-

macrophylli Hansf. 1957

Meliola gesneriae $\mathrm{F}$.

Stevens 1917

Meliola dicranochaeta

Syd. 1926

Asteridiella winteri

(Speg.) Hansf. 1961

Meliola cestri Tehon

1919

Meliola cestricola F.

Stevens 1928

Asteridiella solanacearum

Hansf. 1957

Asteridiella duboisiae

(Hansf.) Hansf. 1961

Asteridiella acervata

(Ellis \& Everh.) Hansf.

1961

Asteridiella laeta

(Theiss.) Hansf. 1961

Asteridiella schwenkiae

(Hansf.) Hansf. 1961

Meliola schwenkiicola

Hansf. 1955

Asteridiella solanacearum

Hansf. 1957

Asteridiella boerhaviifolii

Bat. \& Peres 1963

Meliola wismarensis $\mathrm{F}$.

Stevens 1928

Asteridiella henningsii

(Beeli) Hansf. 1961

Asteridiella adelphica

(Syd.) Hansf. 1961

Meliola

mahabaleshwarensis

Srinivas. 1974

Irenopsis solani $(\mathrm{F}$.

Stevens) F. Stevens 1927

Meliola wismarensis $\mathrm{F}$.

Stevens 1928

Asteridiella solanacearum

Hansf. 1957

!Meliola solanicola

Gaillard 1892

Asteridiella laeta

(Theiss.) Hansf. 1961

Asteridiella plebeja

(Speg.) Hansf. 1961

Meliola canfacotensis

Hansf. 1961

Meliola colombiensis

Hansf. 1955

Meliola kartaboensis F.

Stevens 1928
Stevens 1916

Hansford

$1957 b$

Stevens 1916

Sydow 1926

Hansford

1961

Tehon 1919

Stevens 1928

Hansford

$1956 \mathrm{~b}$

Hansford

1961

Hansford

1961

Hansford

1961

Hansford

1961

Hansford

1955

Hansford

$1956 \mathrm{~b}$

Index

Fungorum

2016

Stevens 1928

Hansford

1961

Hansford

1961

Srinivasulu

1974

Stevens 1927

Stevens 1928

Hansford

$1956 b$

Gaillard 1892

Hansford

1961

Hansford

1961

Hansford

1961

Hansford

1955

Stevens 1928 
Solanum sp.

?Solanum sp.

Solanum stramoniifolium

Solanum terminale

Solanum torvum

Solanum trachycyphum

Solanum verbascifolium*

Solanum verbascifolium*

Solanum viburnum\#

Solanum viride*

Stachyuraceae

Staphyleaceae

Stemonaceae

Stemonuraceae

Stilbaceae

Strelitziaceae

Styracaceae
Stachyurus chinensis

Stachyurus himalaicus

Turpinia formosana

Turpinia occidentalis (Turpinia paniculata)

Turpinia pomifera

Turpinia sp.

Turpinia sp.

Stemona tuberosa

Gomphandra coriacea

Gomphandra coriacea

Gomphandra tetrandra (Stemonurus tetrandrus) Anastrabe integerrima

Nuxia congesta (Lachnopylis congesta)

Nuxia congesta (Nuxia tomentosa)

Nuxia floribunda

Phenakospermum guyannense (Urania guyannensis)

Alniphyllum pterospermum
Meliola pauciseta Hansf. 1955

Asteridiella gaillardii

Hansf. 1957

Irenopsis arimensis

Hansf. 1957

Asteridiella goughiana

Mibey 1997

Irenopsis solani $(\mathrm{F}$.

Stevens) F. Stevens 1927

Asteridiella naucina

(Syd.) Hansf. 1961

Asteridiella winteri

(Speg.) Hansf. 1961

Asteridiella winteri

(Speg.) Hansf. 1961

Asteridiella solani

McAlpine 1897

Asteridiella solani

McAlpine 1897

Appendiculella stachyuri

Y.X. Hu \& B. Song 1993

Asteridiella stachyuri

Ouyang \& B. Song 1993

Appendiculella turpiniae

(W. Yamam.) Hansf.

1961

Irenopsis portoricensis F. Stevens 1927

Stevens 1927

Appendiculella turpiniae Hansford

(W. Yamam.) Hansf.

1961

Meliola oligomera Syd. \& Sydow \&

P. Syd. 1917

Meliola staphyleacearum

Hosag. 1991

Sydow 1917a

Hosagoudar

1991c

Meliola stemonae Syd. Sydow 1922b

1922

Amazonia gomphandrae Hosagoudar

Hosag. 1988

Meliola gomphandrae

Hosag. \& T.K. Abraham 1999

$1988 b$

Hosagoudar

\& Abraham

1999b

Meliola stemonuri Hosag. Hosagoudar 1990

Asteridiella peglerae

(Doidge) Hansf. 1961

Asteridiella nuxiae (Syd.)

Hansf. 1961

\& Goos 1990a

Hansford

1961

Hansford

1961

Asteridiella nuxiae (Syd.) Hansford

Hansf. 1961

1961

Asteridiella nuxiae (Syd.) Hansford

Hansf. 1961

1961

Meliola musae (Kunze ex

Fr.) Mont. 1856

Index

Fungorum

2016

Meliola alniphylli $\mathrm{W}$.

Yamam. 1940
Yamamoto

1940 
Styrax argenteus

Styrax benzoin*

Styrax benzoin*

Styrax formosanus

Styrax leprosus

Styrax serrulatus

Styrax sp.

Styrax sp.

Styrax suberifolius

Symplocaceae Symplocos anamallayana*

Symplocos anamallayana*

Symplocos cochinchinensis var. laurina (Symplocos theophrastifolia)

Symplocos lucida

Symplocos macrocarpa*

Symplocos macrophylla*

Symplocos setchuenensis\#

Symplocos sp.

Symplocos sp.

Symplocos sp.

Symplocos sp.

Symplocos sp.

Symplocos sp.

Symplocos stellaris

(Symplocos eriobotryifolia)

Symplocos tanakae*

Symplocos theiformis
Meliola styracearum F. Stevens 1928

Stevens 1928

Appendiculella Hansford styracicola (W. Yamam.) 1961

Hansf. 1961

Meliola kweichowensis Hansford

Hansf. $1948 \quad 1948$

Appendiculella Hansford

styracicola (W. Yamam.) 1961

Hansf. 1961

Meliola styracina Hansf. Hansford

1957

$1956 b$

Meliola kweichowensis Hansford

Hansf. $1948 \quad 1948$

Asteridiella aberrans (F. Hansford

Stevens) Hansf. $1961 \quad 1961$

Asteridiella tomentosa (G. Hansford

Winter) Hansf. $1961 \quad 1961$

Meliola styracis W. Index

Yamam. 1941

Fungorum

2016

Asteridiella

kodaikanalensis Hosag. et al. 2009

Asteridiella

Hosagoudar et

al. $2009 b$

Hosagoudar et shenbaganurensis Hosag. al. $2009 \mathrm{~b}$ et al. 2009

Meliola symplocicola W. Yamamoto

Yamam. 1941

$1941 b$

Meliola symplocacearum

W. Yamam. 1941

!Meliola rachammae

Hosag. et al. 2010

Asteridiella symploci-

macrophyllae Hosag. \&

Sabeena 2011

Meliola symplocicola W.

Yamam. 1941

Yamamoto

1941b

Hosagoudar et al. 2010a

Hosagoudar

\& Sabeena

2011a

Yamamoto

1941b

Amazonia karnatakensis

Hosag. \& Manian 1989

Amazonia symploci

Hosag. 2013

Hosagoudar

\& Manian

1989

Hosagoudar

2013b

Hansford

1961

(Hansf.) Hansf. 1961

Meliola bissei Schmied.

1989

Schmiedeknec

ht 1989

Meliola maerenhoutiana

Berk. \& Broome 1873

Berkeley \&

Broome 1874

Meliola vittalii Hosag. et al. 2005

Biju et al.

2005

Meliola symploci $\mathrm{W}$.

Yamam. 1940

Yamamoto

1940

Meliola tanakaeana I.

Hino \& Katum. 1957

Hino \&

Katumoto

1957

Asteridiella fidelis (Toro) Hansford

Hansf. 1963

1962 
Symplocos theiformis

Tapisciaceae

Taxaceae

Tapiscia sp.

Tapiscia $\mathrm{sp}$.
Austrotaxus spicata

Taxus canadensis

Taxus cuspidata

Taxus sp.

Camellia japonica

Camellia sinensis (Thea sinensis)

Camellia sinensis (Thea

sinensis)

Gordonia obtusa

Gordonia obtusa

Gordonia obtusa

Gordonia sp.

Gordonia sp.

Pyrenaria microcarpa

(Tutcheria microcarpa)

Pyrenaria microcarpa

(Camellia shinkoensis)

Schima noronhae

Schima sp.

Schima superba (Schima

kankaoensis)

Daphnopsis americana subsp. caribaea (Daphnopsis

caribaea)

Daphnopsis sp.

Gnidia glauca (Lasiosiphon eriocephalus)

Pavetta triflora (Wikstroemia foetida)

Peddiea africana

Peddiea fischeri

Peddiea fischeri
Asteridiella fidelis (Toro) Hansford Hansf. 1963

1962

!Asteridiella tapisciicola $\mathrm{Hu}$ 1990a

H. $\mathrm{Hu} 1990$

Meliola tapisciicola $\mathrm{H} . \quad \mathrm{Hu}$ 1990a

$\mathrm{Hu} 1990$

Asteridiella schmidii B. Index

Huguenin 1969

Fungorum

2016

Asteridiella pitya (Sacc.) Hansford

Hansf. 1961

Asteridiella taxi (Sawada) Müller \&

Hansf. ex E. Müll. \& S.K. Bose 1959

Bose 1959

Asteridiella pitya (Sacc.) Hansford

Hansf. $1961 \quad 1961$

Meliola camelliae (Catt.) Saccardo

Sacc. $1882 \quad 1882$

Asteridiella theae M.S. Patil \&

Patil \& Maham. $1999 \quad$ Mahamulkar

1999

Meliola emespatilii

Hosag. et al. 2006

Appendiculella shettyi

Hosag. et al. 2005

Hosagoudar et

al. 2006a

Biju et al.

2005

Appendiculella Biju et al.

vivekananthanii Hosag. et 2005

al. 2005

Meliola gordoniae Hosag. Hosagoudar 1996

1996

Amazonia gordoniicola Biju et al

Hosag. et al. $2005 \quad 2005$

Irenopsis gordoniae Biju et al.

Hosag. et al. $2005 \quad 2005$

Meliola hosagoudarii B. Song 2003

Song 2003

Meliola camelliicola W. Yamamoto

Yamam. $1941 \quad$ 1941b

Meliola schimae Hansf. Hansford

1954

1954

Meliola theacearum F. Stevens 1928

Stevens 1928

Meliola schimicola W. Yamamoto

Yamam. 1940

1940

Hansford

Asteridiella aibonitensis 1961

Asteridiella aibonitensis Hansford

(F. Stevens) Hansf. 19611961

Irenopsis mysorensis

Hansf. \& Thirum. 1948

Amazonia wikstroemiae

Hansf. 1957

Asteridiella peddieae

(Doidge) Hansf. 1961

Asteridiella masakensis

(Hansf.) Hansf. 1961

Hansford \&

Thirumalacha r 1948

Hansford

$1957 b$

Hansford

1961

Hansford

1961

Meliola peddiicola Hansf. Hansford 1947
$1947 b$ 
Wikstroemia meyeniana

Wikstroemia sp.

Torricelliaceae

Trigoniaceae

Ulmaceae

Urticaceae

Torricellia tiliifolia*

Trigonia sp.

Chaetachme aristata

Achudemia javanica
(Chaetachme microcarpa)

Achudemia javanica

Boehmeria macrophylla

(Boehmeria platyphylla)

Boehmeria macrophylla

(Boehmeria platyphylla)

Cecropia longifera\#

Cecropia peltata

Cecropia peltata

Cecropia peltata (Cecropia arachnoidea)

Cecropia peltata (Cecropia arachnoidea)

Cecropia $\mathrm{sp}$

Cecropia sp.

Coussapoa sp.

Coussapoa sp.

Elatostema angolensis\#

Leucosyke capitellata*

Myriocarpa longipes

Oreocnide integrifolia

Oreocnide sp.

Pilea parietaria

Pilea sp.

Pilea sp.
Asteridiella

wickstroemiae Petr. 1959

Meliola wikstroemiicola

Hansf. 1958

Meliola torricelliae A.K.

Kar \& Maity 1970

Irenopsis moelleriana $(\mathrm{G}$.

Winter) F. Stevens 1927

Meliola chaetachmes

Hansf. 1938

Meliola achudemiae

Hansf. 1954

Meliola anacolosae

Hansf. 1954

Irenopsis boehmeriae

S.N. Ghosh et al. 2005

Meliola platyphyllae Jana et al. 2005

Appendiculella

tonkinensis (P. Karst. \&

Roum.) Toro 1927

Appendiculella echinus

(Henn.) Höhn. 1919

Appendiculella

tonkinensis (P. Karst. \&

Roum.) Toro 1927

Appendiculella

tonkinensis (P. Karst. \&

Roum.) Toro 1927

Appendiculella

tonkinensis (P. Karst. \&

Roum.) Toro 1927

Asteridiella cecropiicola Hansford

(Hansf.) Hansf. 1961

Asteridiella obesa (Speg.)

Hansf. 1961

Appendiculella cornu-

caprae (Henn.) Höhn.

1919

Appendiculella echinus

(Henn.) Höhn. 1919

Meliola thomasiana Sacc.

1905

Asteridiella leucosykes

(H.S. Yates) Hansf. 1961

Asteridiella villebruneae

(Hansf.) Hansf. 1961

Asteridiella

oreocnideicola Hosag.

2006

Irenopsis oreocnidae

Hansf. 1958

Asteridiella pileae

(Hansf.) Hansf. 1961

Asteridiella triloba (G.

Winter) Hansf. 1961

Meliola earlii F. Stevens

1916
Petrak 1958

Hansford

$1957 \mathrm{c}$

Index

Fungorum

2016

Stevens 1927

Hansford

1938

Hansford

1954

Hansford

1954

Jana et al.

$2005 b$

Jana et al.

2005c

Toro 1927

Höhnel 1919

Toro 1927

Toro 1927

Toro 1927

1961

Hansford

1961

Höhnel 1919

Höhnel 1919

Index

Fungorum

2016

Hansford

1961

Hansford

1961

Hosagoudar

\& Biju 2006

Hansford

1957c

Hansford

1961

Hansford

1961

Stevens 1916 
Pipturus arborescens

Villebrunea scabra

Velloziaceae Barbacenia purpurea

Verbenaceae

Citharexylum caudatum

Citharexylum ilicifolium

Citharexylum poeppigii

Citharexylum sp.

Citharexylum spinosum

(Citharexylum fruticosum)

Citharexylum subthyrsoideum

Duranta erecta (Duranta

repens)

Duranta sp.

Lantana horrida (Lantana

hispida)

Lantana sp.

Lantana sp.

Lippia sp.

Phyla nodiflora (Lippia nodiflora)

Phyla nodiflora (Lippia nodiflora)

Stachytarpheta cayennensis

Stachytarpheta cayennensis (Valerianoides cayennensis) Stachytarpheta indica

Stachytarpheta jamaicensis

Stachytarpheta strigosa

Verbena officinalis (Verbena domingensis)

Violaceae

Vitaceae
Rinorea angustifolia subsp. ardisiiflora (Rinorea ardisiiflora)

Rinorea formicaria (Alsodeia formicaria)

Rinorea natalensis*

Cayratia pedata
Asteridiella pipturi

(Hansf.) Hansf. 1961

Asteridiella villebruneae

(Hansf.) Hansf. 1961

Asteridiella barbaceniae

(Hansf.) Hansf. 1961

Meliola verbenacearum

Mibey 1997

Asteridiella vilis (Syd. \& P. Syd.) Hansf. 1961

\#Meliola lagerheimii

Gaillard 1891

Asteridiella citharexyli

Henn. 1902

Asteridiella longipoda

(Gaillard) Hansf. 1961

Asteridiella vilis (Syd. \&

P. Syd.) Hansf. 1961

Asteridiella vilis (Syd. \&

P. Syd.) Hansf. 1961

Asteridiella pittieri (Toro)

Hansf. 1961

Meliola durantae Gaillard

1892

Meliola lantanae Syd. \&

P. Syd. 1914

Meliola ambigua Pat. \&

Gaillard 1888

Meliola sakawensis Henn.

1904

Meliola lippiae Maubl.

1903

Meliola ambigua Pat. \&

Gaillard 1888

Meliola durantae Gaillard 1892

Asteridiella callista

(Rehm) Hansf. 1961

Asteridiella valerianae

Hansf. 1957

Asteridiella callista

(Rehm) Hansf. 1961

Asteridiella callista

(Rehm) Hansf. 1961

Asteridiella callista

(Rehm) Hansf. 1961

Irenopsis verbenae (Cif.)

J.L. Crane \& A.G. Jones 2001

Asteridiella rinoreae

(Doidge) Hansf. 1961

Irenopsis macrochaeta

(Syd. \& P. Syd.) F.

Stevens 1927

Asteridiella rinoreae

(Doidge) Hansf. 1961

Meliola cayratiae Hosag. et al. 2010
Hansford

1961

Hansford

1961

Hansford

1961

Mibey \&

Hawksworth

1997

Hansford

1961

Hansford

$1956 b$

Hennings

1902

Hansford

1961

Hansford

1961

Hansford

1961

Hansford

1961

Gaillard 1892

Fuhrmann \&

Mayor 1914

Patouillard \&

Gaillard 1888

Hennings

1904a

Maublanc

1903

Patouillard \&

Gaillard 1888

Gaillard 1892

Hansford

1961

Hansford

1956b

Hansford

1961

Hansford

1961

Hansford

1961

Crane \&

Jones 2001

Hansford

1961

Stevens 1927

Hansford

1961

Hosagoudar et

al. 2010c 
Cissus adnata

Cissus alata (Cissus

rhombifolia)

Cissus antarctica

Cissus caesia

Cissus erosa

Cissus producta

Cissus repanda

Cissus repanda

Cissus rhomboidea*

Cissus sp.

Cissus sp.

Leea aequata

Leea asiatica

Leea indica

Leea macrophylla

Leea sp.

Rhoicissus revoilii

Tetrastigma harmandii*

Tetrastigma sp.

Vitis flexuosa (Vitis parvifolia)

Vitis montevidensis\#

Vitis negundinis\#

Vitis rheedei*

Vitis umbrosa\#

Winteraceae Drimys sp.

Drimys winteri

Drimys winteri var. chilensis (Drimys chilensis)

Drimys winteri var. chilensis

(Drimys chilensis)

Xanthorrhoeacea Dianella odorata
Meliola merrillii Syd. \& P. Syd. 1913

Meliola cissi-

rhombifoliae Hansf. 1957

Meliola cissi-antarcticae

Hansf. 1953

Meliola cissi-caesiae

Hansf. \& Deighton 1948

Asteridiella perspicua

(Cif.) Hansf. 1961

Meliola cissi-productae

Deighton 1951

Meliola cissi-repandae

Hansf. 1954

Meliola furcata Lév. 1846

Meliola varia Doidge

1917

Meliola cissi Hansf. 1937

Meliola corazoyensis

Hansf. 1961

Irenopsis leeae Hansf.

1937

Meliola maredumilliana

Hosag. \& M. Mohanan

1996

Irenopsis leeae Hansf.

1937

Amazonia leeae Hansf. \&

Thirum. 1948

Asteridiella leeicola

Hansf. 1958

Meliola furcata Lév. 1846

Asteridiella tetrastigmatis

Petr. 1959

Meliola bakeri Syd. \& P.

Syd. 1916

Meliola rizalensis Syd. \&

P. Syd. 1914

Meliola cantareirensis

Hansf. 1957

Irenopsis aciculosa (G.

Winter) F. Stevens 1927

Meliola vitis Hansf. 1947

Meliola campylopoda

Syd. 1926

Asteridiella crustacea

(Speg.) Hansf. 1961

Asteridiella werdermannii

(Hansf.) Hansf. 1961

Meliola corallina Mont.

1850

\#Asterina compacta Lév.

1845

Meliola gregoriana $\mathrm{F}$. Stevens 1925

Stevens 1925
Sydow \&

Sydow 1914b

Hansford

$1957 \mathrm{~b}$

Hansford

1953a

Hansford \&

Deighton

1948

Hansford

1961

Deighton

1951

Hansford

1954

Léveillé 1846

Doidge 1917

Hansford

1937

Hansford

1961

Hansford

1937

Hosagoudar

1996

Hansford

1937

Hansford \&

Thirumalacha r 1948

Hansford

$1957 \mathrm{c}$

Léveillé 1846

Petrak 1958

Sydow \&

Sydow 1916b

Sydow \&

Sydow 1914a

Hansford

$1956 b$

Stevens 1927

Hansford

1947a

Sydow 1926

Hansford

1961

Hansford

1961

Gay 1850

Léveillé 1845 


\begin{tabular}{|c|c|c|c|}
\hline Zingiberaceae & Renealmia aromatica (Alpinia & $\begin{array}{l}\text { Hosag. et al. } 2010 \\
\text { Meliola monopla Cif. }\end{array}$ & $\begin{array}{l}\text { al. } 2010 \mathrm{c} \\
\text { Ciferri } 1954\end{array}$ \\
\hline \multirow[t]{2}{*}{ Zygophyllaceae } & $\begin{array}{l}\text { aromanca) } \\
\text { Balanites aegyptiaca }\end{array}$ & $\begin{array}{l}\text { Meliola balanitis Hansf. } \\
1945\end{array}$ & $\begin{array}{l}\text { Hansford } \\
1945 b\end{array}$ \\
\hline & Guaiacum sanctum & $\begin{array}{l}\text { Meliola capilligera } \text { Cif. } \\
1933\end{array}$ & Ciferri 1933 \\
\hline - & - & $\begin{array}{l}\text { !Meliola araliae Speg. } \\
1888\end{array}$ & $\begin{array}{l}\text { Spegazzini } \\
1888 \mathrm{~b}\end{array}$ \\
\hline - & - & $\begin{array}{l}\text { !Meliola triseptata Berk. } \\
\& \text { Broome }\end{array}$ & $\begin{array}{l}\text { Index } \\
\text { Fungorum } \\
2016\end{array}$ \\
\hline - & - & $\begin{array}{l}\text { Appendiculella rimbachii } \\
\text { (Pat.) Hansf. } 1961\end{array}$ & $\begin{array}{l}\text { Hansford } \\
1961\end{array}$ \\
\hline - & - & $\begin{array}{l}\text { Appendiculella } \\
\text { tuberculata (F. Stevens) } \\
\text { Toro } 1925\end{array}$ & Toro 1925 \\
\hline - & - & $\begin{array}{l}\text { Asteridiella ampullifera } \\
\text { (G. Winter) Hansf. } 1961\end{array}$ & $\begin{array}{l}\text { Hansford } \\
1961\end{array}$ \\
\hline - & - & $\begin{array}{l}\text { Asteridiella boni } \\
\text { (Gaillard) Hansf. } 1961\end{array}$ & $\begin{array}{l}\text { Hansford } \\
1961\end{array}$ \\
\hline - & - & $\begin{array}{l}\text { Asteridiella conglomerata } \\
\text { (G. Winter) Hansf. } 1961\end{array}$ & $\begin{array}{l}\text { Hansford } \\
1961\end{array}$ \\
\hline - & - & $\begin{array}{l}\text { Asteridiella pleurostyliae } \\
\text { (Berk. \& Broome) Hansf. } \\
1961\end{array}$ & $\begin{array}{l}\text { Hansford } \\
1961\end{array}$ \\
\hline - & - & $\begin{array}{l}\text { Asteridiella subcrustacea } \\
\text { (Speg.) Hansf. } 1961\end{array}$ & $\begin{array}{l}\text { Hansford } \\
1961\end{array}$ \\
\hline - & - & $\begin{array}{l}\text { Asteridiella venezuelensis } \\
\text { (Syd.) Hansf. } 1961\end{array}$ & $\begin{array}{l}\text { Hansford } \\
1961\end{array}$ \\
\hline - & - & $\begin{array}{l}\text { Cryptomeliola orbicularis } \\
\text { (Berk. \& M.A. Curtis) S. } \\
\text { Hughes \& Piroz. } 1997\end{array}$ & $\begin{array}{l}\text { Mibey \& } \\
\text { Hawksworth } \\
1997\end{array}$ \\
\hline - & - & $\begin{array}{l}\text { Irenopsis acanthopoda } \\
\text { (Pat.) Hansf. } 1961\end{array}$ & $\begin{array}{l}\text { Hansford } \\
1961\end{array}$ \\
\hline - & - & $\begin{array}{l}\text { Irenopsis curvata (H.S. } \\
\text { Yates) F. Stevens } 1927\end{array}$ & Stevens 1927 \\
\hline - & - & $\begin{array}{l}\text { Irenopsis guignardii } \\
\text { (Gaillard) F. Stevens } 1927\end{array}$ & Stevens 1927 \\
\hline - & - & $\begin{array}{l}\text { Meliola acamptinga Speg. } \\
1908\end{array}$ & $\begin{array}{l}\text { Spegazzini } \\
1908\end{array}$ \\
\hline - & - & $\begin{array}{l}\text { Meliola albonitensis } \mathrm{F} . \\
\text { Stevens } 1916\end{array}$ & Stevens 1916 \\
\hline - & - & $\begin{array}{l}\text { Meliola aliena Syd. \& P. } \\
\text { Syd. } 1912\end{array}$ & $\begin{array}{l}\text { Sydow \& } \\
\text { Sydow 1912b }\end{array}$ \\
\hline - & - & $\begin{array}{l}\text { Meliola andina Gaillard } \\
1892\end{array}$ & Gaillard 1892 \\
\hline - & - & $\begin{array}{l}\text { Meliola balansae Gaillard } \\
1892\end{array}$ & Gaillard 1892 \\
\hline - & - & $\begin{array}{l}\text { Meliola caxangaensis } \\
\text { Bat. } 1963\end{array}$ & $\begin{array}{l}\text { Batista et al. } \\
1963\end{array}$ \\
\hline - & - & $\begin{array}{l}\text { Meliola conferta Doidge } \\
1917\end{array}$ & Doidge 1917 \\
\hline - & - & $\begin{array}{l}\text { Meliola dichotoma Berk. } \\
\& \text { M.A. Curtis } 1860\end{array}$ & $\begin{array}{l}\text { Berkeley \& } \\
\text { Curtis } 1860\end{array}$ \\
\hline- & - & $\begin{array}{l}\text { Meliola forbesii Gaillard } \\
1892\end{array}$ & $\begin{array}{l}\text { Index } \\
\text { Fungorum } \\
2016\end{array}$ \\
\hline
\end{tabular}


Meliola formosa Welw. \& Index

Curr. 1868

Fungorum

2016

Meliola fuscidula Gaillard Index

1892

Fungorum

2016

Meliola guaranitica Speg. Spegazzini 1884

1884

Meliola heterodonta Syd. Sydow \&

\& P. Syd. 1916

Sydow $1916 b$

Meliola insignis Gaillard 1892

Index

Fungorum

2016

Meliola leopoldina

Theissen 1914

Theiss. 1914

Meliola leptopus Theiss. Theissen 1914

1914

Meliola leptospora

Gaillard 1892

Index

Fungorum

2016

Meliola ludibunda Speg. Index 1884

Fungorum

2016

Meliola mattogrossensis

Starbäck 1904

Starbäck 1904

Meliola megalopoda Syd.

\& P. Syd. 1917

Sydow \&

Meliola monilispora

Sydow 1917a

Gaillard 1892

Meliola nidulans

Gaillard 1892

Cooke 1882

(Schwein.) Cooke 1882

Meliola pachytricha (Link Saccardo ex Fr.) Sacc. 1882

Meliola penicillata Lév. Léveillé 1846

1846

Meliola pennata Höhn. Höhnel 1909b 1909

Meliola perexigua

Gaillard 1892

Index

Fungorum

2016

Meliola pulveracea Speg. Spegazzini 1881

1881

Meliola samarensis H.S. Yates 1917

Yates 1917

Meliola spinigera Speg. Spegazzini

1881

1881

Meliola tahitensis Pat. ex

Hansf. 1961

Hansford

Meliola thollonis Gaillard 1892

Index

Fungorum

2016

Meliola wainioi Pat. 1890 Index

Fungorum

2016

Meliola woodiana Sacc.

1899

Index

Fungorum

???

!Meliola litseae $f$.

rotundipoda Săvul. \&

Rayss

2016

Index

Fungorum

2016 


\begin{tabular}{|c|c|c|c|}
\hline$? ? ?$ & $? ? ?$ & $\begin{array}{l}\text { Meliola macarangae } \\
\text { Hansf. } 1954\end{array}$ & Ciferri 1954 \\
\hline$? ? ?$ & $? ? ?$ & $\begin{array}{l}\text { !Meliola polytricha Speg. } \\
1879\end{array}$ & $\begin{array}{l}\text { MycoBank } \\
2016\end{array}$ \\
\hline$? ? ?$ & $? ? ?$ & $\begin{array}{l}\text { !Meliola rodriguezii } \\
\text { Hosag. } 2010\end{array}$ & $\begin{array}{l}\text { Index } \\
\text { Fungorum } \\
2016\end{array}$ \\
\hline$? ? ?$ & $? ? ?$ & $\begin{array}{l}\text { !Meliola roureae H.S. } \\
\text { Yates } 1918\end{array}$ & Yates 1918 \\
\hline$? ? ?$ & $? ? ?$ & $\begin{array}{l}\text { Amazonia tehoni Toro } \\
1932\end{array}$ & $\begin{array}{l}\text { Seaver et al. } \\
1932\end{array}$ \\
\hline$? ? ?$ & $? ? ?$ & $\begin{array}{l}\text { Asteridiella heritiericola } \\
\text { (Thite \& D.K. Kulk.) } \\
\text { Hosag. } 1996\end{array}$ & $\begin{array}{l}\text { Hosagoudar } \\
1996\end{array}$ \\
\hline$? ? ?$ & $? ? ?$ & $\begin{array}{l}\text { Irenopsis caseariae } \\
\text { Hansf. } 1957\end{array}$ & $\begin{array}{l}\text { Hansford } \\
1957 b\end{array}$ \\
\hline$? ? ?$ & $? ? ?$ & $\begin{array}{l}\text { Irenopsis } \\
\text { cynophallophorae Bat. \& } \\
\text { A.F. Vital } 1956\end{array}$ & $\begin{array}{l}\text { Index } \\
\text { Fungorum } \\
2016\end{array}$ \\
\hline$? ? ?$ & $? ? ?$ & $\begin{array}{l}\text { Meliola atricapilla } \\
\text { Starbäck } 1904\end{array}$ & $\begin{array}{l}\text { Index } \\
\text { Fungorum } \\
2016\end{array}$ \\
\hline$? ? ?$ & $? ? ?$ & $\begin{array}{l}\text { Meliola carolinensis A.H. } \\
\text { Sm. \& Hesler } 1940\end{array}$ & $\begin{array}{l}\text { Index } \\
\text { Fungorum } \\
2016\end{array}$ \\
\hline$? ? ?$ & $? ? ?$ & $\begin{array}{l}\text { Meliola caymanensis Ellis } \\
\text { \& Everh. } 1900\end{array}$ & $\begin{array}{l}\text { Index } \\
\text { Fungorum } \\
2016\end{array}$ \\
\hline$? ? ?$ & $? ? ?$ & $\begin{array}{l}\text { Meliola citharexyli } \\
\text { (Hansf.) Cif. } 1961\end{array}$ & Ciferri 1961 \\
\hline$? ? ?$ & $? ? ?$ & $\begin{array}{l}\text { Meliola guariella Hansf. } \\
1957\end{array}$ & $\begin{array}{l}\text { Hansford } \\
1957 b\end{array}$ \\
\hline$? ? ?$ & $? ? ?$ & $\begin{array}{l}\text { Meliola littoralis Syd. } \\
1928\end{array}$ & $\begin{array}{l}\text { Doidge \& } \\
\text { Sydow } 1928\end{array}$ \\
\hline$? ? ?$ & $? ? ?$ & $\begin{array}{l}\text { Meliola oligopoda Syd. } \\
1926\end{array}$ & Sydow 1926 \\
\hline$? ? ?$ & $? ? ?$ & $\begin{array}{l}\text { Meliola petiolaris Petr. } \\
1921\end{array}$ & Petrak 1921 \\
\hline ??? & $? ? ?$ & $\begin{array}{l}\text { Meliola petrakii } \mathrm{F} . \\
\text { Stevens \& Roldan } 1935\end{array}$ & $\begin{array}{l}\text { Stevens \& } \\
\text { Roldan } 1935\end{array}$ \\
\hline$? ? ?$ & $? ? ?$ & $\begin{array}{l}\text { Meliola quinqueseptata } \\
\text { Rehm } 1881\end{array}$ & $\begin{array}{l}\text { Index } \\
\text { Fungorum } \\
2016\end{array}$ \\
\hline$? ? ?$ & $? ? ?$ & $\begin{array}{l}\text { Meliola viticola Hansf. } \\
1945\end{array}$ & $\begin{array}{l}\text { Hansford } \\
1945 b\end{array}$ \\
\hline
\end{tabular}

“*”: unresolved plant species, “\#”: needs to be verify, "?": questioned, “_"”: undetermined, "???”: no information.

Table 2 Excluded names from Index Fungorum.

\begin{tabular}{|c|c|}
\hline Fungal species & Note \\
\hline $\begin{array}{l}\text { Asteridiella daphniphylli W. Yamam. } \\
1956\end{array}$ & Should be Asterina daphniphylli W. Yamam. 1956 \\
\hline Meliola abjecta J. Schröt. & Lack of protologue \\
\hline Meliola acaciae-binervatae Hansf. & Lack of protologue \\
\hline
\end{tabular}


Meliola barbonica Mibey

Meliola bicornis Speg.

Meliola citri Penz.

Meliola foliae-ravenalae

Nascim

Meliola furcata Berl. \& Roum.

Meliola fuscopulveracea Toro

Meliola haplochaeta Syd

Meliola hudsoniana A.H. Sm. 1941

Meliola linhartiana Petr. 1950

Meliola macrocarpa Mont.

Meliola nephitidae Hansf.

Meliola palmae Schwein.

Meliola penicillata Speg.

Meliola petiotariicola Mibey

Meliola tubaraoensis Hansf.
Lack of protologue

Lack of protologue

Lack of protologue

Bat. \& Lack of protologue

Lack of protologue

Lack of protologue

Should be Meliolinopsis haplochaeta (Syd. and P. Syd.)

Beeli 1920

Should be Mycena hudsoniana A.H. Sm. 1941

Lack of protologue

Lack of protologue

Lack of protologue

Lack of protologue

Lack of protologue

Lack of protologue

Lack of protologue

Meliola wyomingensis A.H. Sm. \& Should be Mycena wyomingensis A.H. Sm. \& Arenb. Arenb. 1941

1941

Meliola xylopiifolii Bat. \& J.L. Bezerra Lack of protologue

Table 3 Conflict spellings of Meliolales species.

\section{Current name Name in protologue}

Appendiculella lozanellae Rodr. Just. \& M. Appendiculella lozanella Rodr. Just. \& M. Piepenbr. 2007 Piepenbr. 2007

Appendiculella photiniicola (W. Yamam.) Appendiculella photinicola (W. Yamam.) Hansf. Hansf. 1961 1961

Asteridiella buddlejae (Hansf.) Hansf. 1961 Asteridiella buddleiae (Hansf.) Hansf. 1961

Asteridiella capparis (Hansf. \& Deighton) Hansf. 1961

Asteridiella capparidis (Hansf. \& Deighton) Hansf. 1961

Asteridiella cassiicola (Bat. \& J. Silva) Hansf. 1961

Asteridiella cassiaecola (Bat. \& J. Silva) Hansf. 1961

Asteridiella entandrophragmatis (Hansf.) Asteridiella entandrophragmae (Hansf.) Hansf. Hansf. 1961 1961

Asteridiella erythroxyli (Cif.) Hansf. 1961 Asteridiella erythroxylonis (Cif.) Hansf. 1961

Asteridiella homaliigena Hosag. et al. 2011 Asteridiella homaligena Hosag. et al. 2011

Asteridiella ingicola (Speg.) Hansf. 1961 Asteridiella ingaecola (Speg.) Hansf. 1961

Asteridiella leucosykes (H.S. Yates) Hansf. Asteridiella leucosykeae (H.S. Yates) Hansf. 1961 1961

Asteridiella linocierae (Syd. \& P. Syd.) Hansf. Asteridiella linocieriae (Syd. \& P. Syd.) Hansf. 1961 1961

Asteridiella melastomatacearum (Speg.) Asteridiella melastomacearum (Speg.) Hansf. Hansf. 1961 1961

Asteridiella phukaniea Hosag. et al. 2009 Asteridiella phukanea Hosag. et al. 2009 
Asteridiella pithecellobii (W. Yamam.) Hansf. 1961

Asteridiella rhaphiolepidis (W. Yamam.) Hansf. 1961

Asteridiella thunbergiae-chrysopis (Hansf. \& Deighton) Hansf. 1961

Asteridiella uncariicola Hansf. 1958

Asteridiella usteri (Hansf.) Hansf. 1963

Asteridiella vacciniicola Hansf. 1958

Asteridiella viticis-negundinis Hosag. et al. Asteridiella viticis-negundoi Hosag. et al. 2011 2011

Irenopsis capparicola Bat. \& A.F. Vital 1955

Irenopsis guignardii (Gaillard) F. Stevens 1927

Irenopsis miconiicola (F. Stevens) F. Stevens 1927

Irenopsis moelleriana (G. Winter) F. Stevens 1927

Meliola acaciarum Speg. 1922

Meliola aglaiae-edulis Jana et al. 2005

Meliola albiziae Hansf. \& Deighton 1948

Meliola allophyli Doidge 1928

Meliola angusta F. Stevens \& Tehon 1926

Meliola anonacearum F. Stevens 1928

Meliola atrovelutina Speg. 1924

Meliola banesensis Syd. \& P. Syd. 1916

Meliola bersamae Hansf. 1937

Meliola capparis Hansf. 1946

Meliola ceriopis Hansf. 1957

Meliola choristylidis Doidge 1927

Meliola coccolobae-nodosae Hansf. 1955

Meliola crenato-furcata Syd. \& P. Syd. 1916

Meliola cupaniae-majoris Bat. 1953

Meliola cylindropoda Doidge 1920

Meliola dioicae M.R. Bhise \& C.R. Patil 2014

Meliola ecuadorensis F. Stevens 1928

Meliola elaeidis F. Stevens 1928

Meliola epithematis F. Stevens \& Roldan 1935

Meliola erycibes Hansf. 1954

Meliola erycibes-paniculatae Hosag. 1990

Meliola erythroxylifoliae Bat. \& A.F. Vital 1953

Meliola euodiae Pat. 1888

Meliola exocarpi H.S. Yates 1918

Meliola fahrenheitiae Hosag. \& T.K. Abraham 1998

Meliola ficium H.S. Yates 1918
Asteridiella pithecolobii (W. Yamam.) Hansf. 1961

Asteridiella rhaphiolepsis (W. Yamam.) Hansf. 1961

Asteridiella thunbergiae-chrysopidis (Hansf. \& Deighton) Hansf. 1961

Asteridiella ucariicola Hansf. 1958

Asteridiella usterii (Hansf.) Hansf. 1963

Asteridiella vacciniiicola Hansf. 1958

Irenopsis capparidicola Bat. \& A.F. Vital 1955

Irenopsis guignardi (Gaillard) F. Stevens 1927

Irenopsis miconieicola (F. Stevens) F. Stevens 1927

Irenopsis molleriana (G. Winter) F. Stevens 1927

Meliola acaciorum Speg. 1922

Meliola aglaiae-eduliae Jana et al. 2005

Meliola albizziae Hansf. \& Deighton 1948

Meliola allophylli Doidge 1928

Meliola angustior F. Stevens \& Tehon 1926

Meliola annonacearum F. Stevens 1928

Meliola atro-velutina Speg. 1924

Meliola banosensis Syd. \& P. Syd. 1916

Meliola barsamae Hansf. 1937

Meliola capparidis Hansf. 1946

Meliola ceriopsidis Hansf. 1957

Meliola chloristylidis Doidge 1927

Meliola coccolobis-nodosae Hansf. 1955

Meliola crenatofurcata Syd. \& P. Syd. 1916

Meliola cupaniae-major Bat. 1953

Meliola cylindripoda Doidge 1920

Meliola dioicae M.R. Bhise et al. 2014

Meliola equadorensis F. Stevens 1928

Meliola elaeis F. Stevens 1928

Meliola epithemae F. Stevens \& Roldan 1935

Meliola erycibis Hansf. 1954

Meliola erycibis-paniculatae Hosag. 1990

Meliola erythroxylifolii Bat. \& A.F. Vital 1953

Meliola evodiae Pat. 1888

Meliola exocarpiae H.S. Yates 1918

Meliola farenheitiae Hosag. \& T.K. Abraham 1998

Meliola fiium H.S. Yates 1918 
Meliola heyneae Hansf. \& Thirum. 1948

Meliola holocalicis Speg. 1924

Meliola hypöestes Hansf. 1947

Meliola irvingae Hansf. 1947

Meliola justiciae Hansf. 1938

Meliola lanceolatosetosa Syd. \& P. Syd. 1914

Meliola livistonae H.S. Yates 1917

Meliola lyoniae F. Stevens 1925

Meliola macalpinei Sacc. \& P. Syd. 1899

Meliola makilingiana Syd. \& P. Syd. 1917

Meliola meliosmae Petr. 1959

Meliola mitragynae Syd. \& P. Syd. 1913

Meliola monanthotaxis Mibey 1997

Meliola montis-fontium B. Huguenin 1969

Meliola neurocalycis Jana et al. 2005

Meliola nigrorufescens Sacc. 1917

Meliola obtusifoliae Mibey 1997

Meliola ochthocosmi Hansf. \& Deighton 1948

Meliola ochthocosmicola Hansf. \& Deighton 1948

Meliola olecranonis F. Stevens \& Tehon 1926

Meliola paulliniifoliae Bat. 1960

Meliola pericampyli W. Yamam. 1941

Meliola peruiferi D.B. Pinho et al. 2012

Meliola petitiae Hansf. 1955

Meliola pithecellobii F. Stevens \& Tehon 1926

Meliola pterocarpi H.S. Yates 1918

Meliola rechingeri Hansf. 1958

Meliola sarcostigmatis Hosag. 1990

Meliola sorindeiae Hansf. \& Deighton 1948

Meliola sterculiicola Hosag. \& P.J. Robin 2011

Meliola tonduzii Speg. 191

Meliola vernalis Pinho et al. 2012

Meliola villaresiicola Speg. 1924

Meliola weigeltii Kunze 1817

Meliola wismarensis F. Stevens 1928

Meliola xylosmicola Garcés 1944

Meliola xylosmicola Hansf. 1955

Meliola ziziphi Hansf. \& Thirum. 1948

!Meliola micromeli F. Stevens \& Roldan 1935

!Meliola micromeli T.K. Jana \& S.N. Ghosh 2010

!Meliola serjaniicola Bat. \& A.F. Vital 1953
Meliola heyniae Hansf. \& Thirum. 1948

Meliola holocalycis Speg. 1924

Meliola hypöestis Hansf. 1947

Meliola irvingiae Hansf. 1947

Meliola justitiae Hansf. 1938

Meliola lanceolato-setosa Syd. \& P. Syd. 1914

Meliola livistoniae H.S. Yates 1917

Meliola lyoni F. Stevens 1925

Meliola mac-alpini Sacc. \& P. Syd. 1899

Meliola maquilingiana Syd.

Meliola meliosmatis Petr. 1959

Meliola mitragynes Syd. \& P. Syd. 1913

Meliola monanthotaxiae Mibey

Meliola montisfontium B. Huguenin 1969

Meliola neurocali Jana et al. 2005

Meliola nigro-rufescens Sacc. 1917

Meliola obtisifoliae Mibey

Meliola octhocosmi Hansf. \& Deighton 1948

Meliola octhocosmicola Hansf. \& Deighton 1948

Meliola oleocranonia F. Stevens \& Tehon

Meliola paullinifolii Bat. 1960

Meliola pericamphyli W. Yamam. 1941

Meliola peruiferae D.B. Pinho et al. 2012

Meliola petitae Hansf. 1955

Meliola pitheclobii F. Stevens \& Tehon 1926

Meliola pterocarpiae H.S. Yates 1918

Meliola richtingeri Hansf. 1958

Meliola sarcostigmae Hosag. 1990

Meliola sorideiae Hansf. \& Deighton 1948

Meliola sterculicola Hosag. \& P.J. Robin 2011

Meliola tonduzi Speg. 191

Meliola vernaliae Pinho et al. 2012

Meliola villaresicola Speg. 1924

Meliola weigeltii var. fraxinifoliae Bat. 1956

Meliola wismarensis var. payoensis Syd. 1939

Meliola xylosmaticola Garcés 1944

Meliola xylosmaticola Hansf. 1955

Meliola zizyphi Hansf. \& Thirum. 1948

!Meliola micromali F. Stevens \& Roldan 1925

!Meliola micromelii T.K. Jana \& S.N. Ghosh 2010

!Meliola serjanicola Bat. \& A.F. Vital 1953

\section{Acknowledgements}

This study was supported by the Science and Technology Foundation of Guizhou Province (No. 
[2012]3173). Saranyaphat Boonmee would like to thank the Thailand Research Fund, project number TRG5880152 and Mae Fah Luang University for a project number 2559A30702006. Putarak Chomnunti would like to thank the National Research Council of Thailand (grant No: 230437).

\section{References}

Anthony P, Holtum J, Jackes B. 2002 - Shade acclimation of rainforest leaves to colonization by lichens. Functional Ecology 16, 808-816.

Baker RED, Dale WT. 1951 - Fungi of Trinidad and Tobago. Mycological Papers 33, 1-123.

Batista AC, Cavalcante WA, Maia H da S. 1965a - Novos binomios do gênero Meliola. Atas do Instituto de Micologia da Universidade do Recife 2, 253-289.

Batista AC, Maia H da S, Lima JA, Souza RG, Peres GEP. 1963 - Alguns Meliola identificados no IMUR. Publicações do Instituto de Micologia da Universidade do Recife 237, 1-68.

Batista AC, Maia H da S. 1957 - Contribuição ao estudo dos fungos Meliolaceae. Anais da Sociedade de Biologia de Pernambuco 15, 445-466.

Batista AC, Nascimento ML, Maia H da S. 1956 - Novas espécies de Meliolaceae de Pernambuco. Publicações do Instituto de Micologia da Universidade do Recife 25, 1-11.

Batista AC, Nascimento ML, Maia H da S. 1960 - Iconografia e estudo descritivo de espécies de Meliolaceae. Atas do Instituto de Micologia da Universidade do Recife 1, $13-51$.

Batista AC, Silva AA, Bezerra JL. 1965b - Balladynocallia n.gen. e outros Meliolaceae. Atas do Instituto de Micologia da Universidade do Recife 2, 215-243.

Batista AC, Vital AF. 1955 - Novos elementos para a taxonomia dos fungos de fumagina. Anais da Sociedade de Biologia de Pernambuco 13, 108-124.

Batista AC. 1946 - Estudos fiotpatológicos. Boletim da Secretaria de Agricultura Indústria e Comércio do Estado de Pernambuco 13, 51-60.

Beeli M. 1920 - Note sur le genre Meliola Fr. Bulletin du Jardin Botanique de l'État à Bruxelles 7, 89-160.

Beeli M. 1927 - Contribution á l'étude de la flore mycologique du Congo. IV. Bulletin de la Société Royale de Botanique de Belgique 60, 75-87.

Berkeley MJ, Broome CE. 1873 - Enumeration of the Fungi of Ceylon. Part II, containing the remainder of the Hymenomycetes, with the remaining established tribes of Fungi. Journal of the Linnean Society of London, Botany 14, 29-140.

Berkeley MJ, Broome CE. 1874 - Enumeration of the fungi of Ceylon. Part II. Botanical Journal of the Linnean Society 14, 29-141.

Berkeley MJ, Curtis MA. 1860 - Characters of new fungi, collected in the North Pacific Exploring Expedition by Charles Wright. Proceedings of the American Academy of Arts and Sciences 4, $111-130$

Berkeley MJ, Curtis MA. 1869 - Fungi Cubenses (Hymenomycetes). Botanical Journal of the Linnean Society 10, 280-392.

Berkeley MJ. 1876 - Notices of North American fungi. Grevillea 4, 141-162.

Berlese AN, Voglino P. 1886 - Sylloge fungorum. Additamenta ad Volumina I - IV, 1-484.

Bhise MR, Patil CR, Salunkhe CB. 2014 - Three new black mildew fungi from Mahabaleshwar, Maharashtra, India. International Journal of Life Sciences 2, 304-310.

Biju CK, Hosagoudar VB, Abraham TK. 2005 - Meliolaceae of Kerala, India - XV. Nova Hedwigia 80, 465-502.

Biju H, Bagool RG. 2004 - Meliolaceous fungi from Kerala state, India. Journal of Mycology and Plant Pathology 34, 801-804. 
Boedijn KB. 1940 - The Mycetozoa, fungi and lichens of the Krakatau group. Bulletin du Jardin Botanique de Buitenzorg 16, 358-429.

Brown WH. 1913 - The phenomenon of fatigue in the stigma of Martynia. The Philippine journal of science C Botany 8, 197-285.

Budathoki U, Singh PN. 1994 - Some new black mildews from Kathmandu Valley. Indian Phytopathology 47, 377-380.

Butin H, Speer EO. 1978 - Über einige parasitische Ascomyceten auf Nadeln der Brasilianischen Araukarie. Sydowia 31, 9-26.

Butin H. 1974 - Drei neue Appendiculella-Arten (Meliolaceae) suf Südamerikanischen Cupressaceen. Phytopathologische Zeitschrift 80, 285-290.

Byl PA. 1926 - Description of some previously unnamed South African fungi II. South African Journal of Science 23, 283-284.

Byl PA. 1928 - Description of some previously unnamed South African fungi IV. South African Journal of Science 25, 185-187.

Castellani E. 1947 - Micromiceti Ethiopici ed Eritrei. Nuovo Giornale Botanico Italiano 53, 211229.

Chardón CE, Miller JH, Müller AS. 1940 - Ascomycetes from the state of Minas Geraes, Brazil. Mycologia 32, 172-204.

Chardón CE, Toro RA. 1934 - Mycological explorations of Venezuela. Monograph Universidad de Puerto Rico Series B 2, 1-353.

Chaudhary RK, Kharwar RN, Budathoki U. 1994 - Three new species of black mildew, Appendiculella Hoeh. from Kathmandu Valley (Nepal). Journal of the Living World 1, 172179.

Ciferri R. 1931 - Mycoflora Domingensis exsiccata. Annales Mycologici 29, 283-299.

Ciferri R. 1933 - Mycoflora Domingensis exsiccata. Annales Mycologici 31, 144-167.

Ciferri R. 1938 - Mycoflora domingensis exsiccata. Annales Mycologici 36, 198-245.

Ciferri R. 1951 - Schedae Mycologicae I-XI. Mycopathologia et Mycologia Applicata 6, 19-27.

Ciferri R. 1954 - Meliolae of Santo Domingo (W.I.). Mycopathologia et Mycologia Applicata 7, 81211.

Ciferri R. 1956 - Mycoflora domingensis exsiccata. Sydowia 10, 130-180.

Ciferri R. 1961 - Mycoflora Domingensis Integrata. Quaderno del Laboratorio Crittogamico del Istituto Botanico dell'Università di Pavia 19, 1-539.

Cooke MC. 1878 - Ravenel's American fungi. Grevillea 6, 129-146.

Cooke MC. 1879 - Natal fungi. Grevillea 8, 69-72.

Cooke MC. 1880 - Exotic fungi. Grevillea 9, 10-15.

Cooke MC. 1882 - The Perisporiaceae of Saccardo's Sylloge Fungorum. Grevillea 11, 35-38.

Cooke MC. 1884 - Some exotic fungi. Grevillea 12, 85-85.

Crane JL, Jones AG. 2001 - Nomenclatural and taxonomic revision in the Meliolaceae. Mycotaxon $77,145-151$.

Crous PW, Gams W, Stalpers JA, Robert V, Stegehuis G. 2004 - MycoBank: an online initiative to launch mycology into the 21st century. Studies in Mycology 50, 19-22.

de Macedo DM, Pinho DB, Barreto RW, Pereira OL, Cuda JP. 2010 - Black mildew fungi (Meliolaceae) associated with Schinus terebinthifolius (Brazilian pepper tree) in Brazil Mycotaxon 114, 429-437.

Deighton FC. 1951 - New African Meliolaceae. Sydowia 5, 1-8.

Deighton FC. 1957 - New African Meliolaceae - II. Sydowia 11, 93-115. 
Deighton FC. 1958 - Five new Meliolas from South-East Asia. Sydowia 11, 40-43.

Deighton FC. 1967 - Nomenclatural notes on some Meliolineae. Sydowia 21, 183-187.

Deighton FC. 1968 - New African Meliolaceae - II. Sydowia 11, 93-115.

Devi TP, Chowdhury PN. 2005 - Additions of the genus Appendiculella from south India. Journal of Mycopathological Research 43, 71-73.

Dilcher DL. 1965 - Epiphyllous fungi from Eocene deposits in western Tennessee, USA. Palaeontographica 116, 1-54.

Doassans JE, Patouillard NT. 1888 - Sur quelques espèces de Meliola nouvelles ou peu connues. Revue Mycologique Toulouse 10, 134-141.

Doidge EM, Sydow H. 1928 - The South African species of the Meliolineae. Bothalia 2, 424-472.

Doidge EM. 1917 - South African Perisporiales. I. Perisporiaceae. Transactions of the Royal Society of South Africa 5, 713-750.

Doidge EM. 1920a - Mycological Notes - I. Transactions of the Royal Society of South Africa 8, $117-143$.

Doidge EM. 1920b - South African Perisporiaceae. IV. New species from the Coast Districts. Transactions of the Royal Society of South Africa 8, 111-115.

Doidge EM. 1920c - South African Perisporiaceae. V. Notes on an interesting collection from Natal. Transactions of the Royal Society of South Africa 8, 137-143.

Doidge EM. 1922 - South African Ascomycetes in the National Herbarium. Part II. Nos. 51-85. Bothalia 1, 65-82.

Doidge EM. 1924 - South African Ascomycetes in the National Herbarium. Part III. Nos. 86-135. Bothalia 1, 195-221.

Doidge EM. 1927 - South African Ascomycetes in the National Herbarium. Part IV. Nos 136-159. Bothalia 2, 229-241.

Doidge EM. 1941 - South African Ascomycetes in the National Herbarium. V. Nos 160-195. Bothalia 4, 193-217.

Doidge EM. 1948 - South African Ascomycetes in the National Herbarium. Part VI. Bothalia 4, 837880.

Earle FS. 1901 - Some fungi from Puerto Rico. Muhlenbergia 1, 10-23.

Earle FS. 1904 - Mycological studies. II. Bulletin of the New York Botanical Garden 3, 289-312.

Ellis JB, Everhart BM. 1895 - New species of fungi. I. Sandwich Island fungi. II. Florida fungi. III. Mexican fungi. Bulletin of the Torrey Botanical Club 22, 434-440.

Farr DF, Rossman AY. 2016 - Fungal Databases, Systematic Mycology and Microbiology Laboratory, ARS, USDA. http://nt.ars-grin.gov/fungaldatabases (accessed 7 August 2016).

Farr ML, Horner HTJr. 1968 - Fungi on Selaginella. Nova Hedwigia 15, 239-283.

Farr ML. 1987 - Amazonian foliicolous fungi. IV. Some new and critical taxa in Ascomycotina and associated anamorphs. Mycologia 79, 97-116.

Fries EM. 1828 - Hymenomycetes Uterini seu Elvellaceae. Systema Mycologicum 2, 1-275.

Fries EM. 1830 - Eclogae fungorum, praecipue ex herbarus germanorum de scriptorum. Linnaea 5, 497-553.

Fuhrmann O, Mayor E. 1914 - Voyage d'exploration scientifique des champignons parasites de Colombie. Mémoires de la Société des Sciences Naturelles de Neuchâtel 5, 432-441.

Gaillard A. 1892 - Le genre Meliola. Bulletin de la Société Mycologique de France 8, 176-188.

Gawande SJ, Agarwal DK, Hosagoudar VB. 2004 - Some new foliicolous ascomycetes from northern India. Indian Phytopathology 57, 287-295.

Gay C. 1850 - Historia Física y Política de Chile. Botánica. Flora Chilena 7, 5-512. 
Gilbert GS, Reynolds DR, Bethancourt A. 2007 - The patchiness of epifoliar fungi in tropical forests: host range, host abundance, and environment. Ecology 88, 575-581.

González Fragoso R, Ciferri R. 1928 - Hongos parasitos y saprofitos de la Republica Dominicana. Publicaciones Estación Agronómica de Moca Serie B 13, 1-17.

Goos R, Andersons J. 1974 - The Meliolaceae of Hawaii. Sydowia 26, 73-80.

Goos RD, Hosagoudar VB. 1998 - Meliola chennaiana sp. nov. and some additional records of fungi from India. Mycotaxon 68, 41-46.

Hafiz Khan MA, Azmatullah, Kafi A. 1955 - A new species of Meliola on Butea monosperma. Biologia Lahore 1, 112-113.

Hansford CG, Deighton FC. 1948 - West African Meliolineae II. Meliolineae collected by F.C. Deighton. Mycological Papers 23, 1-79.

Hansford CG, Thirumalachar MJ. 1948 - Fungi of south India. Farlowia 3, 285-314.

Hansford CG. 1937 - Contributions towards the fungus flora of Uganda- I. The Meliolineae of Uganda. Botanical Journal of the Linnean Society 51, 265-284.

Hansford CG. 1938 - Contributions towards the fungus flora of Uganda II. Meliolineae - supplement. Botanical Journal of the Linnean Society 51, 537-545.

Hansford CG. 1941 - Contributions towards the fungus flora of Uganda - III. Some Uganda ascomycetes. Proceedings of the Linnean Society London 153, 4-52.

Hansford CG. 1944 - Contribution towards the fungus flora of Uganda VI. New records. Proceedings of the Linnean Society London 156, 102-124.

Hansford CG. 1945a - Contribution towards the fungus flora of Uganda VII. New records and revisions. Proceedings of the Linnean Society London 157, 20-41.

Hansford CG. 1945b - Nouvelles espèces de champignons récoltés au Congo Belge. Publications de l'Institut Agronomique du Congo Belge 2, 35-53.

Hansford CG. 1946 - Contributions towards the fungus flora of Uganda VIII. New records. Proceedings of the Linnean Society London 157, 138-212.

Hansford CG. 1947a - New or interesting tropical fungi I. Proceedings of the Linnean Society London 158, 28-50.

Hansford CG. 1947b - New tropical fungi II. Proceedings of the Linnean Society London 159, 2142.

Hansford CG. 1948 - Chinese fungi collected by S.Y. Cheo. Farlowia 3, 269-284.

Hansford CG. 1949 - Tropical fungi - III. New species and revisions. Proceedings of the Linnean Society of London 160, 116-153.

Hansford CG. 1953a - Australian fungi. New species and revisions 1. The Meliolaceae of Australia. Proceedings of the Linnean Society of New South Wales 78, 51-82.

Hansford CG. 1953b - Tropical fungi - IV. New species and revisions. Proceedings of the Linnean Society London 165, 166-177.

Hansford CG. 1954 - Meliolales from Indonesia. Reinwardtia 3, 75-112.

Hansford CG. 1955 - Tropical fungi V. New species and revisions. Sydowia 9, 1-88.

Hansford CG. 1956a - Australian Fungi III. New species and revisions. Proceedings of the Linnean Society of New South Wales 81, 23-51.

Hansford CG. 1956b - Tropical fungi - VI. New species and revisions. Sydowia 10, 41-100.

Hansford CG. 1957a - A new species of Amazonia. Kew Bulletin 12, 410-410.

Hansford CG. 1957b - Tropical fungi VII. Beihefte zur Sydowia 1, 86-122.

Hansford CG. 1957c - Tropical fungi VIII. Sydowia 11, 44-69.

Hansford CG. 1961 - The Meliolineae, a monograph. Beihefte zur Sydowia 2, 1-806. 
Hansford CG. 1962 - The Meliolineae supplement. Sydowia 16, 302-323.

Hennings P. 1893 - Fungi Brasiliensis II. Botanische Jahrbücher für Systematik Pflanzengeschichte und Pflanzengeographie 17, 523-525.

Hennings P. 1895a - Fungi goyazenses. Hedwigia 34, 88-116.

Hennings P. $1895 \mathrm{~b}$ - Neue und interessante Pilze aus dem Königl. botanischen Museum in Berlin III. Hedwigia 34, 10-13.

Hennings P. 1897 - Beiträge zur Pilzflora Sudamerikas II. Hedwigia 36, 190-246.

Hennings P. 1898 - Fungi centro-africani. Hedwigia 37, 283-289.

Hennings P. 1900a - Fungi japonici. Botanische Jahrbücher für Systematik Pflanzengeschichte und Pflanzengeographie 28, 259-280.

Hennings P. 1900b - Fungi Paraenses I. Hedwigia 39, 76-80.

Hennings P. 1902 - Fungi S. Paulensis I. a cl. Puttemans Collecti. Hedwigia 41, 104-118.

Hennings P. 1904a - Einige neue Pilze aus Japan. Hedwigia 43, 140-146.

Hennings P. 1904b - Fungi Amazonici a cl. Ernesto Ule collecti III. Hedwigia 43, 351-400.

Hino I, Katumoto K. 1957 - Notes on fungi from western Japan (2). Bulletin of the Faculty of Agriculture Yamaguchi University 8, 637-648.

Hino I, Katumoto K. 1958 - Illustrationes fungorum bambusicolorum VI. Bulletin of the Faculty of Agriculture Yamaguchi University 9, 877-908.

Hirata K. 1972 - Host range and geographic distribution of Erysiphaceae as viewed from the families of Angiosperms, and comparison with Meliolineae. Sydowia 25, 100-118.

Höhnel F von. 1907 - Fragmente zur Mykologie (III. Mitteilung, Nr. 92 bis 155). Sitzungsberichte der Kaiserlichen Akademie der Wissenschaften Math.-naturw. Klasse Abt. I 116, 83-162.

Höhnel F von. 1909a - Fragmente zur Mykologie: VI. Mitteilung (Nr. 182 bis 288). Sitzungsberichte der Kaiserlichen Akademie der Wissenschaften Math.-naturw. Klasse Abt. I 118, 275-452.

Höhnel F von. 1909b - Fragmente zur Mykologie: VII. Mitteilung (Nr. 289 bis 353). Sitzungsberichte der Kaiserlichen Akademie der Wissenschaften Math.-naturw. Klasse Abt. I 118, 813-904.

Höhnel F von. 1909c - Fragmente zur Mykologie: VIII. Mitteilung (Nr. 354 bis 406). Sitzungsberichte der Kaiserlichen Akademie der Wissenschaften Math.-naturw. Klasse Abt. I 118, 1157-1246.

Höhnel F von. 1919 - Fragmente zur Mykologie. XXIII Mitteilung, Nr. 1154 bis 1188. Sitzungsberichte der Kaiserlichen Akademie der Wissenschaften Math.-naturw. Klasse Abt. I 128, 535-625.

Hongsanan S, Tian Q, Peršoh D, Zeng XY et al. 2015 - Meliolales. Fungal Diversity 74, 91-141.

Hosagoudar VB, Abraham TK, Biju CK, Ponnuswamy P. 2003a-Meliola samaderae sp. nov. from Kerala. Indian Phytopathology 56, 295-296.

Hosagoudar VB, Abraham TK, Biju CK. 1999a - Two new Meliola species from Kerala, India. Journal of Mycopathological Research 37, 93-94.

Hosagoudar VB, Abraham TK, Biju CK. 2001a - Meliolaceae of Kerala, India - IX. Journal of Economic and Taxonomic Botany 25, 553-559.

Hosagoudar VB, Abraham TK, Crane CL. 1999b - Meliolaceae of Kerala, India - VI. Mycotaxon $71,149-154$.

Hosagoudar VB, Abraham TK, Crane JL. 1998a - Meliolaceae of Kerala - V. Mycotaxon 69, 391398.

Hosagoudar VB, Abraham TK, Crane JL. 1998b - The identity and nomenclature of Meliola atalantiae. Mycotaxon 66, 419-420.

Hosagoudar VB, Abraham TK, Goos RD. 1997a - Three new species of the Meliolaceae from Kerala, 
India. Mycotaxon 63, 493-496.

Hosagoudar VB, Abraham TK, Goos RD. 1998c - Meliolaceae of Kerala, India - II. Mycotaxon 66, $103-108$.

Hosagoudar VB, Abraham TK, Goos RD. 1998d - Meliolaceae of Kerala, India - III. Mycotaxon 66, 109-113.

Hosagoudar VB, Abraham TK, Goos RD. 1998e - Meliolaceae of Kerala, India - IV. Mycotaxon 66, $115-119$.

Hosagoudar VB, Abraham TK, Krishnan PN, Vijayakumar K. 1997b - Biochemical changes in the leaves of ebony tree affected with black mildew. Indian Phytopathology 50, 439-440.

Hosagoudar VB, Abraham TK. 1996 - Two new Meliolaceae members from Kerala, India. Kavaka $24,15-17$.

Hosagoudar VB, Abraham TK. 1998a - Some interesting Meliolaceaeous fungi from. Kerala India.

Journal of Mycopathological Research 36, 95-103.

Hosagoudar VB, Abraham TK. 1998b - Some Meliolaceae from Kerala, India. Sydowia 50, 14-20.

Hosagoudar VB, Abraham TK. 1999a - New and interesting Meliolaceae members from Keral.

Indian Phytopathology 51, 301-303.

Hosagoudar VB, Abraham TK. 1999b - Some interesting members of the Meliolaceae from Kerala, India. Nova Hedwigia 68, 477-487.

Hosagoudar VB, Agarwal DK, Archana GR. 2006a - A new species of the genus Meliola from Tamil Nadu. Indian Phytopathology 59, 261-262.

Hosagoudar VB, Agarwal DK, Biju H, Archana GR. 2006b - Additions to Meliolaceae from India. Indian Phytopathology 59, 345-350.

Hosagoudar VB, Agarwal DK, Biju H, Archana GR. 2007a - Meliolaceae of Kerala, India - XXIV. Indian Phytopathology 60, 82-87.

Hosagoudar VB, Antony VT. 1988 - Two new species of Meliolaceae from south India. Journal of the Swamy Botanical Club 5, 73-75.

Hosagoudar VB, Archana GR, Agarwal DK. 2007b - Meliolaceae of Kerala, India - XXV. Indian Phytopathology 60, 237-243.

Hosagoudar VB, Archana GR, Kandavel K. 2007c - Meliola exacigena sp. nov. from Kodaikanal, Tamil Nadu, India. Scientific Transactions in Environment and Technovation 1, 53-53.

Hosagoudar VB, Archana GR, Khaleel KM, Soumya M. 2011a-Meliola kannurensis sp. nov. from

Kannur, Kerala. Bioscience Discovery 2, 348-349.

Hosagoudar VB, Archana GR, Rajendraprasad M, Nazarudeen A. 2009a - A new Meliola species from Idukki, Kerala, India. Journal of Threatened Taxa 1, 347-347.

Hosagoudar VB, Archana GR. 2009 - Host range of meliolaceous fungi in India. Journal of Threatened Taxa 1, 269-282.

Hosagoudar VB, Archana GR. 2009 - Meliolaceae of Kerala, India - XXVIII. Journal of Threatened Taxa 1, 348-350.

Hosagoudar VB, Archana GR. 2010 - Meliolaceae of Kerala, India - XXXI. Journal of Threatened

Taxa 2, 889-891.

Hosagoudar VB, Biju CK, Abraham TK, Agarwal DK. 2003b - Meliolaceae of Kerala, India - XI. Indian Phytopathology 56, 102-104.

Hosagoudar VB, Biju CK, Abraham TK, Crane JL. 2000a - Meliolaceae of Kerala, India - VII. Mycotaxon 76, 299-304.

Hosagoudar VB, Biju CK, Abraham TK. 2000b - Meliolaceae of Kerala, India - VIII. Journal of Economic and Taxonomic Botany 24, 474-480. 
Hosagoudar VB, Biju CK, Abraham TK. 2001b - Meliolaceae of Kerala, India - X. Journal of Economic and Taxonomic Botany 25, 68-74.

Hosagoudar VB, Biju CK, Rajkumar G. 2001c - A new species of Meliola (Ascomycetes: Meliolales) from Kerala, India. Zoos' Print Journal 16, 595-596.

Hosagoudar VB, Biju H, Anu Appaiah KA. 2006c - Studies on Foliicolous fungi - XX. Microfungi of Coorg, Karnataka. Journal of Mycopathological Research 44, 1-25.

Hosagoudar VB, Biju H, Appaiah KAA. 2005 - Foiar fungal parasites of Western Ghats found on the plants of sacred groves in Daksina Kannada and Udugi districts of Karnataka. Journal of Mycopathological Research 43, 203-209.

Hosagoudar VB, Biju H. 2006 - Studies on Foliicolous fungi - XXII. Microfungi of Silent Valley Naltional Park, Kalghat District in Kerala State. Journal of Mycopathological Research 44, 39-48.

Hosagoudar VB, Braun U. 1989 - A new black mildew from India. Cryptogamic Botany 1, 56-57. Hosagoudar VB, Dayal BR, Goos RD. 1993 - Meliolaceae of southern India - XII. Mycotaxon 46, 201-210.

Hosagoudar VB, Divya B. 2013 - Additions to Black Mildews. International Journal of Biology, Pharmacy and Allied Sciences 2, 430-438.

Hosagoudar VB, Fathima B. 2013 - Novel Taxa of Black Mildews from Kerala State. Asian Journal of Science and Technology 4, 006-008.

Hosagoudar VB, Goos RD. 1989 - Meliolaceous fungi from the state of Kerala, India I. Mycotaxon $36,221-247$.

Hosagoudar VB, Goos RD. 1990a - Meliolaceous fungi from the state of Kerala, India II. The genus Meliola. Mycotaxon 37, 217-272.

Hosagoudar VB, Goos RD. 1990b - Meliolaceae of South India - VII. Mycotaxon 37, 403-411.

Hosagoudar VB, Goos RD. 1991 - Meliolaceae of South India - X. Mycotaxon 42, 125-147.

Hosagoudar VB, Goos RD. 1996 - Some foliicolous fungi from southern India. Mycotaxon 59, 149166.

Hosagoudar VB, Jagath Thimmaiah C, Jayashankara M. 2011b - Five Asteridiella species from Kodagu, Karnataka, India. Mycosphere 2, 611-615.

Hosagoudar VB, Kaveriappa KM, Raghu PA, Goos RD. 1994a - Meliolaceae of Southern India XVI. Mycotaxon 51, 107-118.

Hosagoudar VB, Mathew SP, Babu D. 2014 - Foliicolous fungi of Andaman Islands. Journal of Threatened Taxa 6, 5447-5463.

Hosagoudar VB, Patil MS, Balakrishnan NP. 1989 - Taxonomic notes on Indian Meliolaceae. Journal of Economic and Taxonomic Botany 13, 78-82.

Hosagoudar VB, Raghu PA, Pillai CM. 1994b - Meliolaceae of southern India - XIV. Nova Hedwigia 58, 529-543.

Hosagoudar VB, Raghu PA. 1993-Meliolaceae of southern India - XV. New Botanist 20, 65-72.

Hosagoudar VB, Rajendran A. 1989 - Meliolaceae of South India - VI. Journal of Economic and Taxonomic Botany 13, 75-77.

Hosagoudar VB, Rajkumar G, Biju CK, Abraham TK. 2001d - Amazonia goniothalami sp. nov. and some additional records of fungi on Goniothalamus wightii from India. Mycotaxon 77, 431433.

Hosagoudar VB, Raju MC. 2013 - Foliicolous fungi of Silent Valley National Park, Kerala, India. Journal of Threatened Taxa 5, 3701-3788.

Hosagoudar VB, Raju VS. 1985 - Taxonomic notes on two species of Meliola Fr. described from 
Maharashtra, India. Journal of Economic and Taxonomic Botany 6, 717-718.

Hosagoudar VB, Ravikumar K, Archana GR. 2009b - Two new Asteridiella species from Tamil Nadu, India. Journal of Threatened Taxa 1, 434-436.

Hosagoudar VB, Ravikumar K, Lakshmanan V. 1992 - A new Meliola species from Madurai district of Tamil Nadu, India. Nova Hedwigia 55, 269-270.

Hosagoudar VB, Riju MC, Agarwal DK. 2010a - Three new Meliolaceae members from Silent Valley National Park. Indian Phytopathology 63, 76-78.

Hosagoudar VB, Riju MC. 2009 - Meliola vatsavayai, a new species from Wayanad, Kerala, India. Indian Journal of Science and Technology 2, 25-25.

Hosagoudar VB, Riju MC. 2010 - Meliolaceae of Kerala, India-XXX. New species and new records. Journal of Threatened Taxa 2, 824-828.

Hosagoudar VB, Riju MC. 2011a - Some interesting Meliolaceae members from Western Ghats

Region of Kerala State. Plant Pathology \& Quarantine 1, 121-129.

Hosagoudar VB, Riju MC. 2011b - Three new fungi from Silent Valley National Park, Kerala, India.

Journal of Threatened Taxa 3, 1615-1619.

Hosagoudar VB, Robin PJ, Archana GR. 2009c - New foliicolous fungi from the campus flora of Madras Christian College, India. Sydowia 61, 243-248.

Hosagoudar VB, Robin PJ, Archana GR. 2010b - Two new black mildews from Mahabaleshwar, Maharashtra, India. Journal of Applied and Natural Science 2, 93-95.

Hosagoudar VB, Robin PJ. 2011a - Five new black mildews from the Western Ghats of Peninsular India. Bioscience Discovery 2, 264-267.

Hosagoudar VB, Robin PJ. 2011b - Four new Meliolaceae (Sordariomycetes: Meliolales) members from Kottayam forests in Kerala State, India. Journal of Threatened Taxa 3, 1782-1787.

Hosagoudar VB, Sabeena A, Archana GR, Thomas J. 2007d - Meliola vallaridis sp. nov. from Kerala, India. Scientific Transactions in Environment and Technovation 1, 69-70.

Hosagoudar VB, Sabeena A, Divya B. 2013 - New fungi from Kerala, India. Journal of Threatened Taxa 5, 4805-4807.

Hosagoudar VB, Sabeena A. 2010 - Two new Meliolaceae members from Kerala, India. Taprobanica 2, 95-96.

Hosagoudar VB, Sabeena A. 2011a - Five new Meliolaceae members from Kerala, India. Bioscience Discovery 2, 117-121.

Hosagoudar VB, Sabeena A. 2011b - Two new and a rare black mildew fungi from Kerala, India. Bulletin of Basic and Applied Plant Biology 1, 49-52.

Hosagoudar VB, Sabeena A. 2012 - New fungi from Western Ghats, India. Plant Pathology \& Quarantine 2, 10-14.

Hosagoudar VB, Sabeena A. 2013a - New black mildews (Ascomycetes) from Kerala State, India.

Journal of Threatened Taxa 5, 3977-3980.

Hosagoudar VB, Sabeena A. 2013b - Three new black mildews from Kerala, India. Journal of Threatened Taxa 5, 4898-4900.

Hosagoudar VB, Sabeena A. 2013c - Two New Black Mildews from Western Ghats of Peninsular India. Global Journal of Science Frontier Research Biological Science 13, 1-2.

Hosagoudar VB, Shiburaj S. 2002 - Meliola gamsii sp. nov. (Ascomycetes, Meliolales) from Kerala, India. Nova Hedwigia 74, 411-413.

Hosagoudar VB, Thomas J, Agarwal DK. 2010c - Additions to Meliolales of India. Nelumbo 52, 18.

Hosagoudar VB, Thomas J, Robin PJ. 2009d - Studies on Foliicolous Fungi - XXX. Fungi of 
Shillong, Meghalaya. Indian Journal of Science and Technology 2, 2-6.

Hosagoudar VB, Thomas J. 2009 - Meliolaceae of Kerala, India - XXXII. Indian Journal of Science and Technology 2, 22-24.

Hosagoudar VB, Thomas J. 2010 - Interesting foliicolous fungi from southern Western Ghats of

Kerala State. Journal of Applied and Natural Science 2, 102-105.

Hosagoudar VB, Udaiyan K, Goos RD. 1994c - Meliola symphorematicola sp. nov. from India.

Mycotaxon 51, 119-121.

Hosagoudar VB. 1987a - Meliolaceae of South India - II. Sydowia 40, 113-121.

Hosagoudar VB. 1987b - Meliola ramacharii sp. nov. from Tamil Nadu, India. Kavaka 15, 5-6.

Hosagoudar VB. 1988a - A new species of 'black mildew' from Andhra Pradesh, India. Journal of

Economic and Taxonomic Botany 12, 246-247.

Hosagoudar VB. 1988b - Meliolaceae of South India - V. Nova Hedwigia 47, 535-542.

Hosagoudar VB. 1988c - Meliolaceae of South India - IV. Indian Botanical Reporter 7, 58-60.

Hosagoudar VB. 1991a - Meliolaceae of South India - VIII. Cryptogamic Botany 2, 183-187.

Hosagoudar VB. 1991b - The genus Armatella (P. Henn.) Theiss. \& Sydow (Meliolaceae) in India.

Journal of Economic and Taxonomic Botany 15, 195-202.

Hosagoudar VB. 1991c - Meliolaceae of South India - IX. Nova Hedwigia 52, 497-503.

Hosagoudar VB. 1994 - Meliolales of India. Journal of Economic and Taxonomic Botany 18, 253-

265.

Hosagoudar VB. 1996 - Meliolales of India. Botanical Survey of India 1-363.

Hosagoudar VB. 2002a - Meliolaceae of Kerala, India XIII. Sydowia 54, 53-58.

Hosagoudar VB. 2002b - Meliolaceae of Kerala, India - XIV. Zoos' Print Journal 17, 747-751.

Hosagoudar VB. 2003a - Meliolaceae of Kerala, India - XII. The genus Meliola on Lecythidaceae

members in India. Persoonia 18, 275-279.

Hosagoudar VB. 2003b - Meliolaceae of Kerala, India - XVII: New species, new variety and new records. Zoos' Print Journal. 18, 1061-1064.

Hosagoudar VB. 2003c - Meliolaceae of Kerala, India - XVIII: New Species, varieties and records.

Zoos' Print Journal 18, 999-1002.

Hosagoudar VB. 2004a - Meliolaceae of Kerala India XIX. Two new species and three new records. Zoos' Print Journal 19, 1390-1393.

Hosagoudar VB. 2004b - Meliolaceae of Kerala, India - XXII. Indian Phytopathology 57, 454-468.

Hosagoudar VB. 2004c - Three new species and one new variety of genus Meliola. Zoos' Print Journal 19, 1462-1465.

Hosagoudar VB. 2005 - Meliolaceae of Kerala, India - XXI. New species and new records. Journal of Mycopathological Research 43, 17-32.

Hosagoudar VB. 2006 - Meliolaceae of Kerala, India - XXIV: Six new species and one new record for India. Zoos' Print Journal 21, 2462-2466.

Hosagoudar VB. 2013a - Meliolales of India - Volume III. Journal of Threatened Taxa 5, 3993-4068.

Hosagoudar VB. 2013b - New and noteworthy black mildews from the Western Ghats of Peninsular India. Plant Pathology \& Quarantine 3, 1-10.

Hosagoudar VB. Archana GR, Sabu T. 2009 - Asteridiella ficicola sp.nov. from Kerala, India. Journal of Threatened Taxa 1, 474-474.

Hosagoudar VB, Manian, S. 1989. A new species of Amazonia Theiss. from Karnataka, India. Journal of Economic and Taxonomic Botany. 13, 45-46

Hughes SJ, Pirozynski KA. 1994 - New Zealand fungi 34. Endomeliola dingleyae, a new genus and new species of Meliolaceae. New Zealand Journal of Botany 32, 53-59. 
Hughes SJ. 1952 - Fungi from the Gold Coast I. Mycological Papers 48, 1-91.

Hughes SJ. 1953 - Fungi from the Gold Coast II. Mycological Papers 50, 1-104.

Hughes SJ. 1978 - New Zealand Fungi 25. Miscellaneous species. New Zealand Journal of Botany $16,311-370$.

Hughes SJ. 1993 - Meliolina and its excluded species. Mycological Papers 166, 1-255.

Huguenin, B. 1969. Micromycètes du Pacifique Sud. VII. Méliolinées de la Nouvelle Calédonie. Revue de Mycologie. 34, 23-61

Hu H, Yang JC. 1989 - Two new speies and four new records of Meliolaceae from Guangdong, China. Acta Mycologica Sinica 8, 86-90.

Hu H. 1990a - A preliminary study of Meliolaceae in Guangdong Province I. Acta Mycologica Sinica 9, 93-99.

$\mathrm{Hu}$ H. $1990 \mathrm{~b}$ - A preliminary study of Meliolaceae in Guangdong Province II. Acta Mycologica Sinica 9, 269-281.

Hu H. 1991c - One new species and one new record of Meliola from Hunan, China. Acta Mycologica Sinica 10, 101-103.

Hu YJ, Lu DJ, Jiang GZ. 1987 - The Meliolaceae of Dinghu Shan Biosphere Reserve. Acta Mycologica Sinica Supplement 1, 77-81.

Hu YX, Lu DJ. 1989 - The Meliolaceae of Hainan province. Acta Mycologica Sinica 8, 180-190.

Hu YX, Ouyang YS, Song B. 1990 - Meliolaceous fungi of the Fujian Province of China. Journal of Fujian College of Forestry 10, 328-334.

Hu YX, Ouyang YS, Song B. 1992 - Three new taxa of the Meliolaceae parasitic on Gymnospermae. Acta Mycologica Sinica 11, 188-194.

Hu YX, Song B, Ouyang YS, Chen ZX. 1993a - A new species from Wuyishan and a new record of Asteridiella for China (family Meliolaceae). Wuyi Science Journal Section C Botany 10, 1-4.

Hu YX, Song B, Ouyang YS. 1993b - New species and new variety of Meliolaceae. Journal of Yunnan Agricultural University 8, 325-332.

Hyde KD, Hongsanan S, Jeewon R, Bhat DJ et al. 2016 - Fungal diversity notes 367-490, taxonomic and phylogenetic contributions to fungal taxa. Fungal Diversity 80, 1-270.

Index Fungorum 2016 - http://www.indexfungorum.org/Names/Names.asp (accessed January 2016). Jana TK, Das AK, Ghosh SN. 2006 - Studies on foliicolous fungi I. Geobios Jodhpur 33, 9-16.

Jana TK, Ghosh SN, Das AK. 2005a - Meliolaceae of Nagaland (India) - II. Journal of Mycopathological Research 43, 33-40.

Jana TK, Ghosh SN, Das AK. 2005b - Meliolaceae of Nagaland India - III. Journal of Mycopathological Research 43, 159-166.

Jana TK, Ghosh SN, Das AK. 2005c - New Meliola Fries from Nagaland (India). Geobios Jodhpur, $32,141-148$.

Jana TK, Ghosh SN, Das AK. 2005d - New Meliolineae from Nagaland (India). Geobios Jodhpur $32,265-274$.

Jana TK, Ghosh SN, Das AK. 2005e - Two new species of Meliola from India. Indian Phytopathology 58, 443-447.

Jana TK, Ghosh SN. 2007 - A new species of Meliola from West Bengal. Journal of Mycopathological Research 45, 277-279.

Jana TK, Ghosh SN. 2008a - A new Appendiculella species from Darjeeling, West Bengal, India. Journal of Mycology and Plant Pathology 38, 517-519.

Jana TK, Ghosh SN. 2008b - A new species of Meliola from West Bengal, India. Journal of Mycology and Plant Pathology 38, 520-522. 
Jana TK, Ghosh SN. 2008c - New Asteridiella species from Darjeeling, West Bengal. Geobios Jodhpur 35, 167-168.

Jana TK, Ghosh SN. 2008d - New Meliola species from Jalpaiguri, West Bengal. Geobios Jodhpur $35,72-74$.

Jana TK, Ghosh SN. 2008e - One new species of Meliola from India. Indian Phytopathology 61, 510-513.

Jana TK, Ghosh SN. 2010 - New Meliola species from West Bengal. Journal of Mycopathological Research 48, 153-154.

Jiang GZ. 1986a - The Meliolaceae of China I. Acta Mycologica Sinica Supplement 1, 65-70.

Jiang GZ. 1986b - The Meliolaceae of China II. Acta Mycologica Sinica Supplement 1, 71-76.

Jiang GZ. 1989 - The Meliolaceae of China III. Acta Mycologica Sinica 8, 169-179.

Jiang GZ. 1995 - The Meliolaceae of China IV. Acta Mycologica Sinica 14, 1-9.

Kalchbrenner K, Cooke MC. 1880 - South African fungi. Grevillea 9, 17-34.

Kamal Singh S, Gupta BK. 1986 - Fungi of Gorakhpur - XL. Indian Journal of Mycology and Plant Pathology 16, 245-248.

Kamal Singh S, Singh RP. 1982 - Fungi of Gorakhpur XX. Indian Journal of Mycology and Plant Pathology 12, 70-73.

Kar AK, Maity MK. 1970a - New Meliolineae from West Bengal (India). Sydowia 24, 59-68.

Kar AK, Maity MK. 1970b - The Pyrenomycetes of West Bengal - I. Nytt Magasin for Botanik 17, 81-89.

Karsten PA, Roumeguère C. 1890 - Champignons nouveaux du Tonkin récemment récoltés par M.B. Balansa. Revue Mycologique Toulouse 12, 75-79.

Katumoto K, Harada Y. 1979 - Plant parasitic fungi from the Bonin Islands II. Ascomycotina and Deuteromycotina. Transactions of the Mycological Society of Japan 20, 411-428.

Katumoto K. 1963 - Notes on fungi from western Japan 6. Journal of Japanese Botany 38, 23-28.

Katumoto K. 1991 - Three ascomycetous fungi from the Ryukyo Islands, Japan. Transactions of the Mycological Society of Japan 32, 37-43.

Katumoto K. 2010 - List of Fungi Recorded in Japan [unknown pagination] Tokyo: Kanto Branch of Mycological Society of Japan.

Koorders SH. 1907 - Botanische Untersuchungen. Verhandelingen Koninklijke Nederlandse Akademie van Wetenschappen Afdeling Natuurkunde 13, 1-264.

Léveillé JH. 1845 - Champignons exotiques. Annales des Sciences Naturelles Botanique 3, 38-71.

Léveillé JH. 1846 - Descriptions des champignons de l'herbier du Muséum de Paris. Annales des Sciences Naturelles Botanique 5, 249-305.

Maia H da S, Batista AC. 1960 - Algumas espécies de Amazonia Theiss. e Amazoniella Bat. \& Maia. Brotéria Série Trimestral: Ciências Naturais 29, 73-84.

Maublanc A. 1903 - Sur quelques espèces nouvelles de champignons inférieurs. Bulletin de la Société Mycologique de France 19, 291-296.

McAlpine D. 1896 - Descriptions of Australian Fungi. Proceedings of the Linnean Society of New South Wales 21, 104-106.

McAlpine D. 1897 - New South Wales Fungi. Proceedings of the Linnean Society of New South Wales 22, 36-47.

Mibey RK, Cannon PF. 1999 - Biotrophic fungi from Kenya. Ten new species and some new records. Cryptogamie Mycologie 20, 249-282.

Mibey RK, Hawksworth DL. 1997 - Meliolaceae and Asterinaceae of the Shimba Hills, Kenya. Mycological Papers 174, 1-108. 
Mibey RK, Kokwaro JO, Mukunya DM. 1996 - Four new species and some new records of Meliolaceous fungi from Kenya. Mycotaxon 57, 87-95.

Mibey RK, Kokwaro JO. 1998 - Meliola icacinacearum and M. kerichoensis, spp. nov. from Kenya. Mycological Research 102, 1418-1420.

Mibey RK, Kokwaro JO. 1999 - Two new species of Meliola from Kenya. Fungal Diversity 2, 153157.

Mibey RK, Kokwaro JO. 2001 - Two new graminicolous Meliolaceae from Kenya. African Journal of Mycology and Biotechnology 9, 1-4.

Montagne JPFC. 1842 - Troisième Centurie de plantes cellulaires exotiques nouvelles, Décades I, II, III et IV. Fungi cubenses. Annales des Sciences Naturelles Botanique 17, 119-128.

Müller E, Bose SK. 1959 - Über eine Erkrankung an Taxus im Himalaya. Indian Phytopathology $12,13-18$.

Nair LN, Kaul VP. 1983 - Indian Meliolineae II. Two new species and a new variety of Meliola. Sydowia 36, 204-207.

Nayar T, Hosagoudar V, Prakashkumar R, Jothish P, Thripthi K. 1998 - The occurrence of Meliolaceae ascospores in the air of Kerala, India. Grana 37, 253-254.

Nithyatharani R, Dhivaharan V, Hosagoudar VB. 2010 - Meliola thiyagesanii sp. nov. from Kodaikanal in Tamil Nadu. Scientific Transactions in Environment and Technovation 3, 169170.

Nithyatharani R, Hosagoudar VB, Dhivaharan V, Riju MC. 2011 - Novel fungi from Kodaikanal, Tamil Nadu, India. Scientific Transactions in Environment and Technovation 4, 164-167.

Old KM, Wingfield MJ, Yuan ZQ. 2003 - A manual of diseases of Eucalyptus in South-East Asia. CIFOR.

Orejuela CG. 1944 - New or heretofore unreported species of the higher ascomycetes from Colombia and Venezuela. Mycologia 36, 429-459.

Ouyang YS, Song B, Hu YX. 1993 - Two new species of the Meliolaceae parasitic on Stachyuraceae. Acta Mycologica Sinica 12, 265-270.

Pande A. 2008 - Ascomycetes of Peninsular India. Alaka Pande.

Patil MS, Mahamulkar SH. 1999 - Studies on Meliolaceae of India - IV. Indian Phytopathology 52 , 245-253.

Patil MS, Thite AN. 1974 - Some Ascomycetes from Maharashtra. Maharashtra Vidnyan Mandir Patrika 9, 104-110.

Patouillard NT, Gaillard A. 1888 - Champignons du Vénézuéla et principalement de la région du Haut-Orénoque, récoltés en 1887 par M. A. Gaillard. Bulletin de la Société Mycologique de France 4, 92-129.

Patouillard NT, Hariot PA. 1908 - Fungorum novorum decas tertia. Bulletin de la Société Mycologique de France 24, 13-16.

Patouillard NT. 1897 - Contributions à la flore mycologique du Tonkin. Journal de Botanique (Morot) $11,335-349$.

Petrak F, Ciferri R. 1930 - Fungi Dominicani. Annales Mycologici 28, 377-420.

Petrak F, Ciferri R. 1932 - Fungi Dominicani II. Annales Mycologici 30, 149-353.

Petrak F. 1921 - Mykologische Notizen III. Annales Mycologici. 19, 176-223.

Petrak F. 1948 - Pilze aus Ekuador. Sydowia 2, 317-386.

Petrak F. 1954 - Beiträge zur Pilzflora von Britisch Nord-Borneo. Sydowia 8, 12-26.

Petrak F. 1955 - Beiträge zur Pilzfora von Süd- und Östasien. Sydowia 9, 530-538.

Petrak F. 1958 - Beiträge zur Kenntnis der Meliolineen. Sydowia 12, 431-465. 
Petrak F. 1962 - Kleine Beiträge zur Südamerikanischen Pilzflora. Sydowia 16, 235-249.

Pinho DB, Firmino AL, Ferreira-Junior WG, Pereira OL. 2012 - An efficient protocol for DNA extraction from Meliolales and the description of Meliola centellae sp. nov. Mycotaxon 122, $333-345$.

Pinho DB, Firmino AL, Ferreira-Junior WG, Pereira OL. 2013 - New Meliolaceae from the Brazilian Atlantic Forest 2: species on host families Annonaceae, Cecropiaceae, Meliaceae, Piperaceae, Rubiaceae, Rutaceae and Tiliaceae. Mycologia 105, 697-711.

Pinho DB, Honorato Junior J, Firmino AL, Hora Junior B et al. 2014 - Reappraisal of the black mildews (Meliolales) on Hevea brasiliensis. Tropical Plant Pathology 39, 89-94.

Pinho DB, Pereira OL, Firmino AL, da Silva M et al. 2012 - New Meliolaceae from the Brazilian Atlantic forest 1. Species on hosts in the families Asteraceae, Burseraceae, Euphorbiaceae, Fabaceae and Sapindaceae. Mycologia 104, 121-137.

Raciboraki VM. 1891 - Ueber einige Pilze aus Südrussland. Hedwigia 30, 243-263.

Rao VG. 1969 - Fungi on citrus from India. Sydowia 23, 215-224.

Rehm H. 1901 - Beiträge zur Pilzflora von Südamerika XIII. Xylariaceae. Hedwigia 40, 141-170.

Rehm H. 1913a - Ascomycetes philippinenses - II. The Philippine Journal of Science Section C Botany 8, 251-263.

Rehm H. 1913b - Ascomycetes philippinenses - III. Philippine Journal of Science Section C Botany 8, 391-406.

Rehm H. 1913c - Ascomycetes philippinenses collecti a clar. C.F. Baker. The Philippine Journal of Science Section C Botany 8, 181-194.

Reynolds D. 2010 - Epifoliar fungi of Singapore. Gardens Bulletin Singapore 61, 401-435.

Rodríguez Hernández M, Camino Vilaró M. 1987 - New species of meliolaceous fungi from Cuba. Feddes Repertorium 98, 509-513.

Rodríguez Justavino D, Kirschner R, Piepenbring M. 2015 - New species and new records of Meliolaceae from Panama. Fungal Diversity 70, 73-84.

Rodríguez Justavino D, Piepenbring M. 2007 - Two new species of Appendiculella (Meliolaceae) from Panama. Mycologia 99, 544-552.

Rodríguez M, Minter DW. 1998 - Meliola guatteriae. IMI Descriptions of Fungi and Bacteria 1354, $1-2$.

Rodríguez M, Minter DW. 1999 - Meliola protiicola. IMI Descriptions of Fungi and Bacteria 1387, $1-2$.

Roumeguère C. 1880 - Fungi Gallici exsiccati, cent. X. Revue Mycologique Toulouse 2, 200-202.

Saccardo PA, Sydow P. 1899 - Supplementum Universale, Pars IV. Sylloge Fungorum 14, 1-1316.

Saccardo PA. 1882 - Sylloge Pyrenomycetum, Vol I. Sylloge Fungorum 1, 1-768.

Saccardo PA. 1917 - Notae mycologicae series XXIII. Fungi Philippinenses. Atti della Accademia Scientifica Veneto-Trentino-Istriana 10, 57-94.

Saccardo PA. 1921 - Notae mycologicae. Series XXIV. I. Fungi Singaporenses Barkesiani. Bolletino dell'Orto Botanico Regia dell'Universita de Napoli 6, 39-73.

Saccardo PA. 1926 - Supplementum Universale, Pars X. Phycomycetae, Laboulbeniomycetae, Pyrenomycetae p.p. Sylloge Fungorum 24, 1-703.

Saenz GS, Taylor JW. 1999 - Phylogenetic relationships of Meliola and Meliolina inferred from nuclear small subunit rRNA sequences. Mycological research 103, 1049-1056.

Sawada K. 1931 - Descriptive catalogue of the Formosan fungi V. Report of the Department of Agriculture Government Research Institute of Formosa 51, 1-131.

Schmiedeknecht M. 1989 - Meliolales aus Cuba. Wissenschaftliche Zeitschrift der Friedrich- 
Schiller-Universität Jena 38, 185-209.

Seaver FJ, Chardón CE, Toro RA, Kern FD. 1932 - Botany of Porto Rico and the Virgin Islands.

Supplement to Mycology. Scientific Survey of Porto Rico and the Virgin Islands 8, 209-240.

Sharma JK, Mohanan C, Florence EJM. 1985 - Disease survey in nurseries and plantations of forest tree species grown in Kerala. KFRI Research Report, Kerala Forest Research Institute 36, 1275.

Soares WRO, Dianese JC. 2014 - New Meliola species on fabaceous hosts from the Brazilian Cerrado. Mycologia 13, 321-331.

Song B, Li TH, Goos RD. 2002a - Three new species of the genus Meliola from China. Mycotaxon $84,79-82$.

Song B, Li TH, He Q. 2000 - A new species of Asteridiella. Mycosystema 19, 470-472.

Song B, Li TH, Hosagoudar VB. 2002b - A new species of the genus Amazonia from China. Mycotaxon 84, 121-123.

Song B, Li TH, Shen YH. 2001 - A new species of Irenopsis. Mycosystema 20, 22-24.

Song B, Li TH, Shen YH. 2002c - Studies on Appendiculella in China IV. Mycosystema 21, 177180.

Song B, Li TH, Shen YH. 2002d - Three new species of Meliola from China. Mycosystema 21, 172176.

Song B, Li TH, Shen YH. 2003 - Two new Meliola species from China. Fungal Diversity 12, 173177.

Song B, Li TH. 2003 - Six taxa of Meliolaceae from Yunnan Province in HKAS, China. Mycotaxon $87,421-424$.

Song B, Li TH. 2004a - Interesting taxa of Meliolaceae in HMAS, China. Mycotaxon 90, 129-132.

Song B, Li TH. 2004b - Studies on the genus Asteridiella of China 2. Mycotaxon 89, 201-204.

Song B, Ouyang YS, Hu YX. 1996a - Studies on Appendiculella in China. Acta Mycologica Sinica $15,93-97$.

Song B, Ouyang YS, Hu YX. 1996b - Studies on the genus Asteridiella of China I. Acta Mycologica Sinica 15, 247-250.

Song B, Ouyang YS, Hu YX. 1997a - New species and new records of Meliola from China I. Mycosystema 16, 102-105.

Song B, Ouyang YS, Hu YX. 1997b - New species and new records of Meliola from China II. Mycosystema 16, 176-178.

Song B, Ouyang YS, Hu YX. 1997c - New species and new records of Meliolaceae (Fungi) in China. Journal of Tropical and Subtropical Botany 5, 33-40.

Song B, Ouyang YS, Hu YX. 1997d - Studies on Appendiculella in China II. Mycosystema 16, $244-$ 246.

Song B, Ouyang YS, Hu YX. 1997e - Two new species of the Meliolaceae in China. Mycosystema $16,9-13$.

Song B. 1998 - Studies on Appendiculella in China III. Mycosystema 17, 214-217.

Spegazzini C. 1881 - Fungi argentini additis nonnullis brasiliensibus montevideensibusque. Pugillus quartus (Continuacion). Anales de la Sociedad Científica Argentina 12, 97-117.

Spegazzini C. 1884 - Fungi Guaranitici. Pugillus 1. Anales de la Sociedad Científica Argentina 17, $119-134$.

Spegazzini C. 1888a - Fungi Fuegiani. Boletín de la Academia Nacional de Ciencias en Córdoba 11, $135-311$.

Spegazzini C. 1888b - Fungi Guarinitici. Pugillus II. Anales de la Sociedad Científica Argentina 26, 


\section{$5-74$.}

Spegazzini C. 1889 - Fungi Puiggariani. Pugillus 1. Boletín de la Academia Nacional de Ciencias en

Córdoba 11, 381-622.

Spegazzini C. 1891a - Fungi guaranitici nonnulli novi vel critici. Revista Argentina de Historia

Natural 1, 398-432.

Spegazzini C. 1891b - Phycomyceteae Argentinae. Revista Argentina de Historia Natural 1, 28-38.

Spegazzini C. 1908 - Fungi aliquot paulistani. Revista del Museo de La Plata 15, 7-48.

Spegazzini C. 1909 - Mycetes Argentinenses. Series IV. Anales del Museo Nacional de Historia

Natural Buenos Aires 19, 257-458.

Spegazzini C. 1910 - Fungi Chilenses. Contribución al Estudio de los Hongos Chilenos. Revista de

la Facultad de Agronomía Universidade Nacional de La Plata 6, 1-205.

Spegazzini C. 1912 - Mycetes argentinenses (Series VI). Anales del Museo Nacional de Historia

Natural Buenos Aires 23, 1-146.

Spegazzini C. 1919a - Fungi Costaricenses nonnulli. Boletín de la Academia Nacional de Ciencias en Córdoba 579, 541-609.

Spegazzini C. 1919b - Reliquiae mycologicae tropicae. Boletín de la Academia Nacional de Ciencias en Córdoba 23, 365-541.

Spegazzini C. 1921 - Mycetes chilenses. Boletín de la Academia Nacional de Ciencias en Córdoba $25,1-124$.

Spegazzini C. 1922 - Micromycetes nonnulli Brasilienses. Anales de la Sociedad Científica Argentina 93, 111-118.

Spegazzini C. 1923 - Honguillos exóticos. Boletín de la Academia Nacional de Ciencias en Córdoba 26, 369-403.

Spegazzini C. 1924 - Revisión de las Meliolas Argentinas. Anales del Museo Nacional de Historia Natural Buenos Aires 32, 339-393.

Srinivasulu BV. 1974 - Genus Meliola from Maharashtra. Beihefte zur Nova Hedwigia 47, 421-437.

Srivastava SL, Topal R. 1981 - Meliola garhwalensis nov. sp. from Garhwal Himalayas, India. Geophytology 11, 264-265.

Starbäck K. 1899 - Ascomyceten der ersten Regnell'schen Expedition I. Bihang till Kungliga svenska Vetenskaps-Akademiens Handlingar 25, 1-68.

Starbäck K. 1904 - Ascomyceten der ersten Regnell'schen Expedition III. Arkiv før Botanik 2, 1-22.

Stevens FL, Roldan EF. 1935 - Philippine Meliolineae. The Philippine Journal of Science Section C Botany 56, 47-80.

Stevens FL, Tehon LR. 1926 - Species of Meliola and Irene from British Guiana and Trinidad.

Mycologia 18, 1-22.

Stevens FL. 1916 - The genus Meliola in Porto Rico. Illinois Biology Monographs 2, 475-554.

Stevens FL. 1925 - Hawaiian fungi. Bulletin of the Bernice Bishop Museum 19, 1-189.

Stevens FL. 1927 - The Meliolineae I. Annales Mycologici 25, 405-469.

Stevens FL. 1928 - The Meliolineae II. Annales Mycologici 26, 167-383.

Stevenson JA. 1943 - Fungi novi denominati - I. Mycologia 35, 629-637.

Stevenson JA. 1946 - Fungi novi denominati - II. Mycologia 38, 524-533.

Subhedar AW, Rao VG. 1977 - Some hitherto undescribed species of Ascomycetes from India. Journal of the University of Poona 50, 25-31.

Sydow H, Petrak F. 1929 - Fungi costaricensis a cl. Prof. Alberto M. Brenes collecti. Annales Mycologici 27, 1-86.

Sydow H, Petrak F. 1937 - Fungi costaricenses a cl. Prof. Alberto M. Brenes collecti. Series secunda. 
Annales Mycologici 35, 73-97.

Sydow H, Sydow P, Butler EJ. 1911 - Fungi Indiae orientalis pars III. Annales Mycologici 9, 372421.

Sydow H, Sydow P. 1904 - Novae fungorum species. Annales Mycologici 2, 162-174.

Sydow H, Sydow P. 1910 - Fungi novi Phillippinenses. Annales Mycologici 8, 36-41.

Sydow H, Sydow P. 1912a - Beschreibungen neuer südafrikanischer Pilze. Annales Mycologici 10, 33-45.

Sydow H, Sydow P. 1912b - Fungi from the island of Palawan. Leaflets of Philippine Botany 5, 1533-1547.

Sydow H, Sydow P. 1912c - Novae fungorum species VII. Annales Mycologici 10, 77-85.

Sydow H, Sydow P. 1913a - Enumeration of Philippine fungi with notes and descriptions of new species, I: micromycetes. The Philippine Journal of Science Section C Botany 8, 265-285.

Sydow H, Sydow P. 1913b - Notes and descriptions of Philippine fungi - II. Leaflets of Philippine Botany 6, 1919-1933.

Sydow H, Sydow P. 1913c - Novae fungorum species - X. Annales Mycologici 11, 254-271.

Sydow H, Sydow P. 1913d - Novae fungorum species XI. Annales Mycologici 11, 402-408.

Sydow H, Sydow P. 1914a - Diagnosen neuer philippinischer Pilze. Annales Mycologici 12, 545576.

Sydow H, Sydow P. 1914b - Enumeration of Philippine fungi with notes and descriptions of new species, II. The Philippine Journal of Science Section C Botany 8, 475-508.

Sydow H, Sydow P. 1914c - Fungi from northern Palawan. Philippine Journal of Science Section C Botany 9, 157-189.

Sydow H, Sydow P. 1914d - Novae fungorum species - XII. Annales Mycologici 12, 195-204.

Sydow H, Sydow P. 1916a - Fungi amazonici a cl. E. Ule lecti. Annales Mycologici 14, 65-97.

Sydow H, Sydow P. 1916b - Weitere Diagnosen neuer philippinischer Pilze. Annales Mycologici 14, 353-375.

Sydow H, Sydow P. 1917 - Beitrag zur Kenntniss der Pilzflora der Philippinen-Inseln. Annales Mycologici 15, 165-268.

Sydow H, Sydow P. 1917b - Novae fungorum species - XV. Annales Mycologici 15, 143-148.

Sydow H, Sydow P. 1921 - Die Pilze Mikronesiens aus der Sammlung Ledermann. Botanische Jahrbücher für Systematik Pflanzengeschichte und Pflanzengeographie 56, 430-432.

Sydow H, Sydow P. 1939 - Fungi aequatorienses. Annales Mycologici 37, 275-438.

Sydow H. 1913 - Beiträge zur Kenntnis der Pilzflora des Südlichen Ostindiens, I. Annales Mycologici 11, 326-330.

Sydow H. 1921 - Novae fungorum species XVII. Annales Mycologici 19, 304-309.

Sydow H. 1922a - Bemerkungen zu einer Anzahl in letzter Zeit als neu beschriebener Pilze der Philippinen-Inseln. Annales Mycologici 20, 66-73.

Sydow H. 1922b - The Amboina fungi collected by C.B. Robinson. The Philippine Journal of Science Section C Botany 21, 131-146.

Sydow H. 1922c - Über einige weitere im sudlichen China (Kwangtung-Provinz) gesammelte Pilze. Annales Mycologici 20, 61-65.

Sydow H. 1923a - Ein neuer Beitrag zur Kenntnis der Pilzflora der Philippinen-Inseln. Annales Mycologici 21, 93-106.

Sydow H. 1923b - Über einige in Britisch Nord-Borneo gesammelte Pilze. Annales Mycologici 21, 89-92.

Sydow H. 1924 - Beschreibungen neuer südafrikanischer Pilze IV. Annales Mycologici 22, 419-435. 
Sydow H. 1925 - Fungi from the Province of Sorsogon. Leaflets of Philippine botany 9, 3113-3138.

Sydow H. 1926 - Fungi in intinere costaricensi collecti, pars secunda. Annales Mycologici 24, 283426.

Sydow H. 1928 - Fungi Borneenses. Annales Mycologici 26, 85-99.

Sydow H. 1930 - Fungi venezuelani. Annales Mycologici 28, 29-224.

Sydow H. 1937 - Neue oder bemerkenswerte australische Micromyceten I. Annales Mycologici 35, 22-49.

Sydow P, Sydow H. 1920a - Novae fungorum species XVI. Annales Mycologici 18, 154-160.

Sydow P, Sydow H. 1920b - Weitere neue Micromyceten der Philippinen-Inseln. Annales Mycologici 18, 98-104.

Tehon LR. 1919 - Studies of some Porto Rican fungi. Botanical Gazette Crawfordsville 67, 501-511. The Plant List 2013 - Version 1.1. Published on the Internet: http://www.theplantlist.org (accessed 1 January 2013).

Theissen F, Sydow H. 1915 - Die Dothideales. Kritisch-systematische Originaluntersuchungen. Annales Mycologici 13, 147-746.

Theissen F. 1910 - Perisporiales riograndenses. Brotéria Série Botânica 9, 5-44.

Theissen F. 1912 - Fragmenta brasilica IV nebst Bemerkungen über einige andere Asterina-Arten. Annales Mycologici 10, 1-32.

Theissen F. 1913 - Über einige Mikrothyriaceen. Annales Mycologici 11, 493-511.

Theissen F. 1914 - Annotacoes à Mycoflora Brasileira. Brotéria Série Botânica 12, 13-31.

Theissen F. 1916 - Beiträge zur Systematik der Ascomyzeten. Annales Mycologici 14, 401-439.

Theissen F. 1919 - Neue Original-Untersuchungen von Askomyceten. Verhandlungen der Zoologisch-Botanischen Gesellschaft Wien 69, 1-24.

Thimmaiah CJ, Hosagoudar VB, Jayashankar M. 2013 - Black mildews of Kodagu, Karnataka, India. Journal of Threatened Taxa 5, 5021-5180.

Thite AN, Patil CR. 1985 - Additions to sooty moulds of Maharashtra. Geophytology 15, 82-86.

Thite AN, Patil SD. 1983 - Studies on Indian Meliolaceae - III. Geophytology 13, 124-126.

Thomas J, Alex TE, Thomas RJ. 2013 - Meliola marthomaensis sp. nov. an Addition to Meliolaceae from Western Ghat Region in Kerala State, India. Universal Journal of Plant Science 1, 100103.

Thümen F von. 1876 - Fungi Austro-Africani IV. Flora (Regensburg) 59, 568-571.

Toro RA. 1925 - New or noteworthy Porto Rican Pyrenomycetes. Mycologia 17, 131-147.

Toro RA. 1927 - Fungi of Santo Domingo - I. Mycologia 19, 66-85.

Toro RA. 1952 - Study of the tropical American black-mildews. Journal of Agriculture of the University of Puerto Rico 36, 24-87.

Verma RK, Sharma N, Soni KK, Jamaluddin. 2008. Forest Fungi of Central India. International Book Distributing Company, Lucknow, India, 1-418.

Viégas AP. 1944 - Alguns fungos do Brazil II. Ascomicetos. Bragantia 4, 1-392.

Viégas AP. 1947 - Notas sobre Meliola pontualli n.sp. Boletim da Secretaria de Agricultura Indústria

e Comércio do Estado de Pernambuco 14, 337-340.

Wellman FL. 1972 - Tropical American plant disease.

Winter G. 1886 - Fungi exotici III. Hedwigia 25, 92-104.

Winter G. 1887 - Rabenhorstii Fungi europaei et extraeuropaei exsiccati cura Dr. G. Winter. Centuria XXXV et XXXVI. Hedwigia 26, 24-35.

Yamamoto W. 1940 - Formosan Meliolineae II-IV. Transactions of the Natural History Society of Formosa 30, 414-425. 
Yamamoto W. 1941a - Formosan Meliolineae III. Transactions of the Natural History Society of Formosa 31, 14-30.

Yamamoto W. 1941b - Formosan Meliolineae IV. Transactions of the Natural History Society of Formosa 31, 47-60.

Yamamoto W. 1941c - Formosan Meliolineae V. Transactions of the Natural History Society of Formosa 31, 125-137.

Yamamoto W. 1957 - Formosan Meliolaceae (Meliolineae) (VII). Science Reports of the Hyogo University of Agriculture 3, 19-22.

Yamamoto W. 1958 - Taxonomy of Meliolaceae with special reference to Formosan species. Science Reports of the Hyogo University of Agriculture 3, 51-93.

Yang JC. 1989 - Preliminary studies on Meliolineae from Guangdong Province of China. Acta Mycologica Sinica 8, 1-8.

Yates HS. 1917 - Some recently collected Philippine fungi. The Philippine Journal of Science Section C Botany 12, 361-380.

Yates HS. 1918 - Some recently collected Philippine fungi, II. The Philippine Journal of Science Section C Botany 13, 361-384.

Zimmermann A. 1901 - Die thierischen und pflanzlichen Feinde der Kautschuk- und GuttaperchaPflanzen. Bulletin de I'lnstitut Botanique de Buitenzorg 10, 1-27. 